\title{
XV Latin-American Symposium on Chronobiology
}

Ana Silva ${ }^{1 *}$

Pablo Torterolo ${ }^{2^{*}}$

${ }^{1}$ Laboratorio de Neurociencias, Facultad de Ciencias, Universidad de la República, Montevideo, Uruguay.

${ }^{2}$ Laboratorio de Neurobiología del Sueño, Departamento de Fisiología, Facultad de Medicina, Universidad de la República, Montevideo, Uruguay.

Corresponding author:

Ana Silva

E-mail: asilva@fcien.edu.uy

Pablo Torterolo

E-mail: ptortero@fmed.edu.uy

DOI: $10.5935 / 1984-0063.20200005$
The XV Latin-American Symposium on Chronobiology (LASC 2019) (www.lasc2019. org) was held in Uruguay, for the first time, from October 7 to 11, 2019, at the Convention Center of the Hotel Mirador in Colonia del Sacramento. It was coordinated by an Organizing Committee with members selected from a group of Uruguayan researchers related to the disciplines of Sleep and Chronobiology, and chaired by Dr. Ana Silva and Dr. Pablo Torterolo. The Organizing Committee was supported by a Scientific Advisory Group composed of prestigious researchers from Argentina, Brazil, Chile, Mexico, and USA.

LASC 2019 was a big success that brought together 198 participants from five Latin American countries, as well as from USA, Germany, Italy, Netherlands, and Israel, with an excellent relationship between senior researchers and students (1-1.4).

The scientific program, available in LASC 2019 website, included four plenary conferences by leading researchers, on topics of disciplines of high impact and importance. Roberto Amici (Bologna University, Italy) presented: "Torpor and sleep: what's the link"; Horacio de la Iglesia (University of Washington-USA): "Sleep: lost and not found"; Gina Poe (University of California- Los Angeles, USA): "Sleep features that optimize adaptive memory reorganization", and Noga Kronfeld-Schor (Tel Aviv University, Israel): "Chronobiology of interspecific interactions in a changing world". LASC 2019 also included eight symposia, five workshops, two poster sessions, and an opening session dedicated to the presentation of works by Latin-American young researchers. There were 63 speakers and 103 posters presented in two sessions. LASC 2019 closed with an outreach final session dedicated to the communication and transfer of chronobiological knowledge to the society (Title of the Round Table: "El reloj en la Sociedad"). Four speakers chaired by Dr. Diego Golombek (Universidad de Quilmes, Argentina) from Argentina (Andrea Pattini, Universidad de Mendoza, and Juliana Leone, Universidad de Quilmes), Brazil (Claudia Moreno, Universidade de Sao Paulo), and Chile (John Ewer, Universidad de Valparaíso) presented examples of the endorsement of social policies based on chronobiological evidence in Latin America.

Before and in parallel with the LASC 2019, the first Latin American School of Chronobiology and Sleep was organized by Dr. Adriana Migliaro, Dr. Alicia Costa and Dr. Luciana Benedetto from the Universidad de la República, Montevideo, Uruguay. This School, conceived as a satellite event to LASC 2019, was an initiative aimed to promote future Latin American chronobiologists to interact among them, and with US and European consolidated scientists. It consisted of two main modules. The first one was held in Montevideo and consisted of foreign and local faculty lectures as well as hands-on training. The second one took place in Colonia del Sacramento, in simultaneous to LASC 2019; it included a data analysis workshop, data blitz sessions and the "meet the experts" dinner. The School was kindly supported by the International Brain Research Organization (IBRO).

Organizing LASC 2019, and the associated School of Chronobiology and Sleep was an enormous challenge for the Uruguayan chronobiology community that had an enormous impact in the consolidation of regional and international collaborations. 
We could not envisage a better corollary to LASC 2019 than the edition of this Special Edition of the Sleep Science Journal, in which we highlight the best poster presentations, whose authors were invited to write and submit a report (original or short review of the research topic). A jury of experts appointed among LASC 2019 participants evaluated all the posters and selected the presentations of young scientists from Argentina,
Brazil, Chile, Mexico, and Uruguay. The selected works not only involve studies on a wide variety of models systems including traditional rodents, native animals, and humans, but also encompass the great diversity of topics and approaches that belong to Chronobiology. Taken together, they emerge as a good picture of the diverse cutting-edge contributions of Latin America Chronobiology. 


\section{Circadian modulation of motivation-related behavior}

Julieta Acosta *

Diego A. Golombek

Patricia V. Agostino

Department of Science and Technology, National University of Quilmes/CONICET, Buenos Aires, Argentina.

\begin{abstract}
Most living organisms have a circadian timing system adapted to optimize the daily rhythm of exposure to the environment. This circadian system modulates several behavioral and physiological processes, including the response to natural and non-natural rewards. Food is the most potent natural reward across species. Food-seeking is known to be mediated by dopaminergic and serotonergic transmission in cortico-limbic pathways. In the present review, we revise the evidence that documents a circadian modulation of reward-related behaviors, with special emphasis in natural rewards. Elucidating the effect of circadian rhythms on motivation behavior may contribute to improve treatment related to psychiatric disorders or drugs of abuse.
\end{abstract}

Keywords: Circadian System; Motivation; Food Reward; Dopamine; Serotonin; Nucleus Accumbens 


\section{INTRODUCTION}

The circadian clock is an endogenous timing system that drives rhythmicity with a period close to 24 -h in almost all living organisms. Some physiological and behavioral functions regulated by the circadian system include the sleep-wake cycle, body temperature, hormone release, and gene expression. For instance, nearly half of all mammalian genes are rhythmically expressed in one or more tissues ${ }^{1}$. Circadian rhythms continue to operate in constant environmental conditions (i.e., free-run), indicating the endogenous nature of circadian rhythms (reviewed $i^{2}$ ). Within the brain, many reward-related areas express circadian clock genes ${ }^{3}$.

Motivation is a complex behavior that pushes us to perform actions to achieve goals ${ }^{4}$. Rewards are recognized to act as hedonic incentives, causing neural representations that elicit motivation and goal pursuit. Appropriate responses to natural rewards (such as food or sex) were evolutionarily important for survival, reproduction, and fitness. At the anatomical level, reward-related behavior emerges from the dynamic activity of neural networks involving several brain structures, such as the nucleus accumbens (NAc), the prefrontal cortex (PFC), the hippocampus (Hipp), the amygdala (AMG), and the thalamus ${ }^{5}$. In addition, it is well known that dopaminergic and serotonergic pathways play an important role in mediating reward processing ${ }^{6}$. Several components of both monoaminergic systems are modulated in a circadian fashion.

In recent years, consistent evidence on circadian regulation of motivation and reward behaviors has emerged as a consequence of its relevance in psychiatric disorders (reviewed $\mathrm{in}^{7,8}$ ) and drug addiction (reviewed in ${ }^{9-11}$ ). In the present review, we will revise the literature concerning the circadian influence in motivation and reward-related behaviors, with a main focus on natural (e.g., food) rewards.

\section{THE CIRCADIAN SYSTEM}

In mammals, the master circadian oscillator is located in the suprachiasmatic nuclei (SCN) of the hypothalamus, and it is mainly synchronized by the light/dark (LD) cycle $^{12}$, which acts together with secondary and peripheral oscillators to keep daily and circadian rhythms. The synchrony or temporal coordination of circadian oscillators between central and peripheral tissues, and their alignment with the external environment, is extremely important for maintaining organism homeostasis ${ }^{13}$.

At the molecular level, circadian oscillations in the SCN are sustained by negative feedback loops of transcriptional and translational processes. In the mammalian circadian system, a primary feedback loop is composed by the positive elements CLOCK and BMAL1, which heterodimerize and promote the transcription of per (Period) and cry (Cryptochrome) genes (negative elements) by acting on specific binding sites (E-box sequences) located in their promoter region. After transcription and translation, PER and CRY proteins heterodimerize and translocate to the nucleus to inhibit their own transcription by acting on the dimer CLOCK/BMAL1 ${ }^{14}$. Each transcriptional/translational loop takes near 24-h to complete.
In addition to this primary loop, another negative feedback loop contributes to clock precision and robustness. In the second loop, the protein REV-ERB $\alpha$ moves to the nucleus to repress bmal1 expression; inversely, $\mathrm{ROR} \alpha$ can stimulate its transcription, both binding to RORE (Retinoic acid-related Orphan receptor Response Element) sites on the bmal1 promoter ${ }^{15}{ }^{16}$. These oscillations of negative and positive elements generate circadian rhythmicity, which regulates the circadian output pathway by driving downstream clock-controlled gene (CCG) expression.

In both SCN cells and non-SCN oscillators cells, the transcriptional/ translational feedback loops involve essentially the same core clock components, although some variations are present. For example, in some brain areas - including the forebrain and the mesolimbic system - the neuronal PAS domain protein 2 (NPAS2) acts as a functional substitute for $\mathrm{CLOCK}^{17,18}$.

The current view of the circadian system implies a hierarchical multi-oscillator network of circadian clocks ${ }^{19}$. This network involves a master oscillator synchronized by light (the $\mathrm{SCN}$ ), additional central oscillators synchronized by non-photic signals (such as food and certain drugs, see below), and peripheral oscillators (in diverse organs and tissues). Additionally, different regions within the brain (referred as secondary oscillators) express rhythmically the core clock genes, and are also capable of sustaining rhythms when they are isolated from the organism and grown in vitro ${ }^{20,21}$. It has been proposed that outside-SCN brain clocks are useful for timing behavioral and physiological tasks to underlie specific activities during the 24-h, such as sleep, vigilance, learning, motivation, vision, or olfaction ${ }^{22}$. In this sense, understanding the role of circadian rhythmicity in extra-SCN brain structures it is relevant not only to elucidate the circadian network functioning as a whole, but also to comprehend how temporal regulation affects the production of complex behaviors.

Although the $\mathrm{SCN}$ is considered to be the main regulator of circadian rhythms, there are at least two central oscillators that are non-photically entrained - the food-entrainable oscillator (FEO) and the methamphetamine-entrainable oscillator (MASCO) - which are independent from the $\mathrm{SCN}^{23,24}$. When food availability is limited to a few hours during each day, mammals quickly alter daily rhythms of physiology and behavior, such as locomotor activity, body temperature, and corticosterone secretion, to correlate with the food availability rhythm. Under these circumstances, a clear behavioral output is food-anticipatory activity (FAA), which implies an increase in locomotor activity that occurs before a daily timed meal. This food-entrainable oscillator is independent of the SCN and still displays clear circadian characteristics. One of its most important features is that FAA persists in the absence of food, suggesting that the FEO is able to generate a sustained free-running rhythm ${ }^{25,26}$. Nevertheless, the anatomical loci of this oscillator is unknown. It is worth noting that at least some features of circadian entrainment (such as non-photic synchronization induced by forced locomotion or feeding) also depend upon reward-related mechanisms, including dopaminergic activation. 


\section{MOTIVATION-RELATED BEHAVIOR}

Although different authors use slightly different definitions of this term, motivation typically refers to the willingness to invest resources (such as time or effort) in order to receive a reward or to avoid a punisher ${ }^{27,28}$. Thus, several behavioral processes are collectively regarded as motivation-related behavior, such as reward and punishment learning, incentive processing, and goaldirected behavior. Different components of motivation have been distinguished ${ }^{29,30}$. The "liking" component implies the experience of pleasure, while the "wanting" component (incentive salience) implies the motivation to obtain it. Although research on this topic includes studies of both aversive and appetitive motivation, the present review focuses only on appetitive behavior.

Several evidences point to dopamine (DA) and serotonin (5-HT) signaling in controlling appetitive motivation and natural reward processes - such as those regulating food and sexual behaviors ${ }^{31}$. Food intake is regulated by complementary homeostatic and hedonic mechanisms. While hypothalamic nuclei mainly regulate the homeostatic drive of feeding, corticolimbic structures control rewarded feeding behaviors ${ }^{9,32}$.
The NAc integrates reward and motivational inputs and translates them for motor outputs. Excitatory glutamatergic inputs carrying information of context, cues, and behavioral control from the PFC, Hipp, AMG, thalamus, and other regions converge onto the NAc (Figure 1). In the NAc, DA mediates these effects through D1-like and D2-like receptors. Although DA appears to be a neural communication system shared by food and drug seeking, the mechanisms involved in these two processes may differ ${ }^{5}$. In support of a role for DA in motivation for food reward, it has been reported that after forebrain DA depletion, animals will cease to actively search for food (and eventually starve to death), but they will still consume food when it is placed in their mouth ${ }^{27}$. It was also found that treatment with DA receptor antagonists reduced responding for food under behaviorally demanding schedules (i.e., when animals have to make a relatively large numbers of responses for food), but not when little or no effort was required to obtain $\mathrm{it}^{27,33}$. In this context, it is generally assumed that $\mathrm{DA}$ is mainly involved in the "wanting" component of motivation ${ }^{27}$.

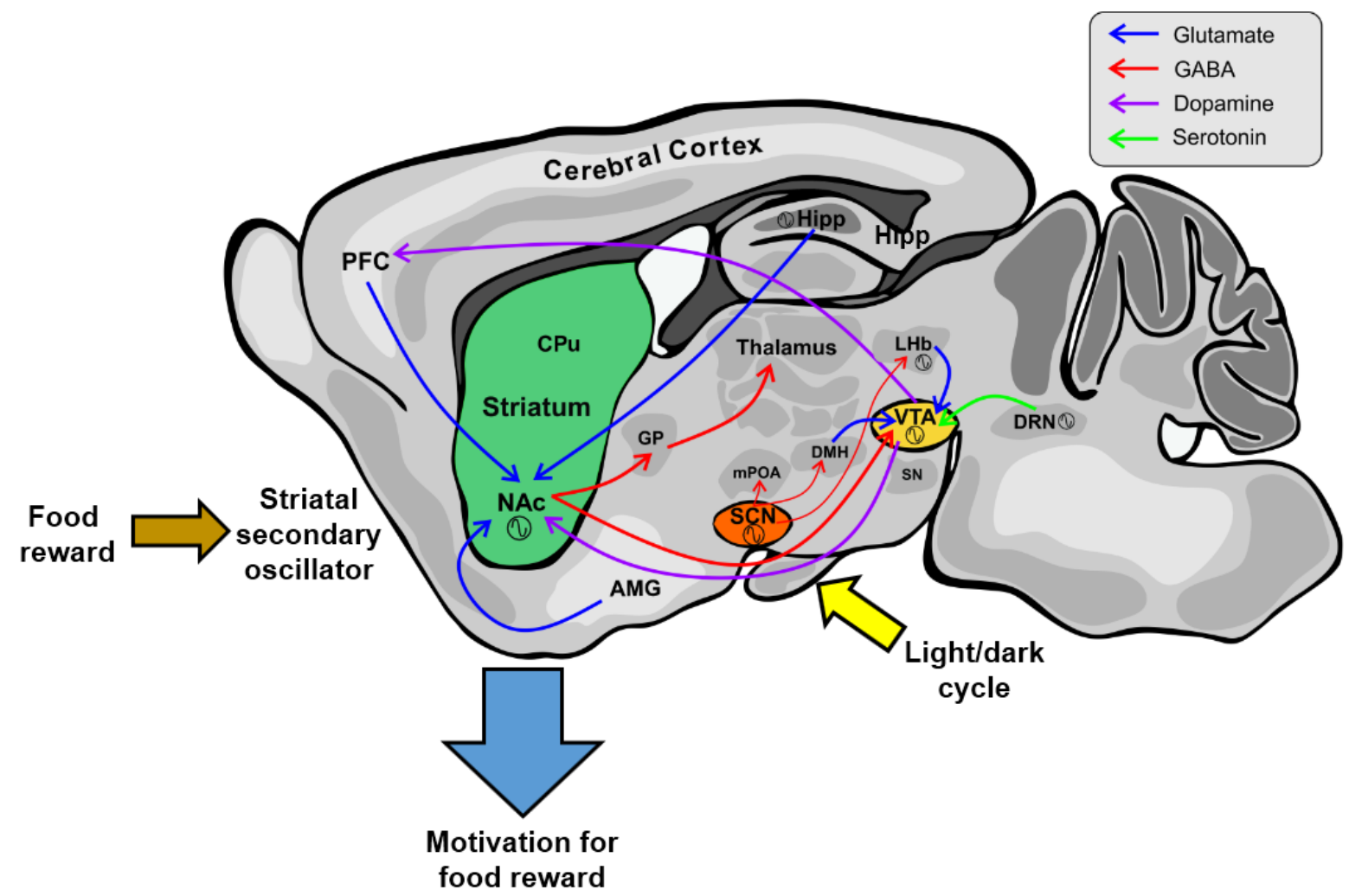

Figure 1. Main brain circuits involved in the circadian modulation of motivation for food reward. The NAc receives glutamatergic inputs from the PFC, AMG, and hippocampus, and provides GABAergic input to the VTA as well as the GP. The VTA also sends afferent inputs to the PFC. Each of these critical extra-SCN brain regions has been shown to maintain rhythms and to express circadian genes and proteins with clock and non-clock regulatory functions. An indirect SCN output circuit may be involved in the circadian regulation of motivated behaviors. The SCN provides direct inputs to $\mathrm{mPOA}, \mathrm{LHb}$ and $\mathrm{DMH}$, which in turn project to the VTA. In addition, DRN neurons receive light information from the circadian visual system and send serotonergic afferents to the VTA. Color lines indicate different neurotransmitter signaling. Abbreviations: AMG, amygdala; $\mathrm{CPu}$, caudate putamen; DMH: dorsomedial hypothalamus; DRN, dorsal raphe nuclei; GABA, gamma-aminobutyric acid; 5-HT, serotonin; GP, globus pallidus; Hipp, hippocampus; LHb, lateral habenula; mPOA, medial pre-optic area; NAc, nucleus accumbens; SCN, suprachiasmatic nuclei; SN, substantia nigra. VTA, ventral tegmental area. Modified from ${ }^{82}$. 
5-HT has also been reported to affect motivation. Selective 5-HT reuptake inhibitors (SSRIs) act on the serotonin transporter (SERT), which is responsible for reuptake and recycling of released 5-HT. Blockade of SERT by SSRIs results in elevation in extracellular 5-HT levels. Several findings showed that SSRIs reduced responding for drug reinforcers, or shifted it in ways that indicated reductions in motivation ${ }^{34}$. In regards to natural rewards, Sanders and collaborators reported an effect of the 5-HT reuptake inhibitor fluoxetine and genetic deletion of SERT on food-reinforced behavior in three paradigms: the progressive ratio (PR) task, which tests the amount of work an animal is willing to perform for food reward, the concurrent food choice operant task, which allows an analysis of effort expended to obtain a preferred reward, and the Pavlovian-to-Instrumental transfer task (PIT), which examines the ability of Pavlovian conditioned cues that predict reward to enhance operant responding. Both fluoxetine treatment and SERT gene deletion reduced operant responding for food reward as well as a generalized reduction in motor output $^{34}$. Moreover, optogenetic stimulation of 5-HT neurons promotes waiting for expected reward, suggesting their role in the reward anticipatory behavior ${ }^{35,36}$. It has been recently reported that 5-HT neurons in the dorsal raphe nuclei (DRN) respond when an animal expects and receives various natural rewards (such as food, sucrose, sex or social interaction), suggesting that the DRN serves as an important reward-processing station in parallel to the midbrain dopamine center ${ }^{37}$.

\section{CIRCADIAN MODULATION OF MOTIVATED BEHAVIOR}

\section{Behavioral circadian modulation.}

At the behavioral level, the response to several types of reinforcers has been reported to be modulated by the circadian system $^{38}$. A large body of evidence reported daily circadian rhythms in self-administration of drugs of abuse, as well as sensitization and locomotor activation after drug intake in rodents (reviewed in ${ }^{9-11}$ ). Regarding the circadian influence in motivation and reward-related behaviors to natural rewards (i.e., sex or food) the existing literature is more limited. For instance, Webb and collaborators reported that sexual performance and mating behavior presented a rhythmic variation throughout the day in rats, with a maximum for sex reward during the middark period of the $\mathrm{LD}$ cycle $^{39}$. In mice, a daily and circadian rhythm in sucrose intake and preference was observed, with greater values during the dark phase. In addition, these rhythms were abolished in the arrhythmic period 2 mutant mice ${ }^{40}$, suggesting that circadian rhythms of hedonic feeding behaviors may be under control of the circadian molecular machinery. It has also been shown that the nuclear receptor RevErb $\alpha$ is important for modulating the orexinergic activity in the lateral hypothalamus of mice in response to food-reward signals ${ }^{32}$.

We recently demonstrated a robust variation in motivation for food reward in young and aged mice by using the PR task. This rhythm was also sustained in constant darkness conditions (DD), suggesting that this variation in motivation is endogenous and constitutes a circadian rhythm.
Surprisingly, we found that the rhythm in motivation is preserved in aged mice. Animals exhibited higher motivation for food reward during the nighttime (their active phase) compared to the daytime (their resting phase). Additionally, circadian arrhythmicity induced by constant light (LL) significantly reduced motivation. We also found that the LD conditions cause a generalized decrease in motivation in both phases tested (day and night), as compared to the DD conditions. Most importantly, we demonstrated that the effect of the alternating LD cycle exerted a more dramatic consequence on the motivated behavior displayed during the day. What's more, the daily and circadian variation on motivation for food reward was maintained in mice without calorie restriction when chocolate was used as a reward. Overall, these results suggest that the circadian system may influence different aspects of motivated and reward-related behaviors, including both the physiological driven states - that promote food consumption and the hedonic aspects associated with feeding ${ }^{41}$.

\section{Circadian modulation of dopaminergic and serotonergic signaling within cortico-limbic areas.}

Numerous studies documented the circadian modulation of DA signaling in different brain areas. For example, circadian regulatory elements were found in the promoter regions of genes expressing the monoamine oxidase $\mathrm{A}(\mathrm{MaoA})^{18}$, the tyrosine hydroxylase $(\mathrm{TH})^{42}$, the dopamine transporter $(\mathrm{DAT})^{43}$, and the dopamine receptor type 3 (DRD3) ${ }^{44}$. More importantly, daily rhythms of expression in almost all the components of dopaminergic metabolism have been documented ${ }^{39}, 45,46$. Moreover, results from our group show that striatal DA levels measured by HPLC-ED present a daily rhythm under LD conditions in mice, with lower levels during the day and a peak during the night ${ }^{47}$. This is consistent with previous reports in rats $^{48,49}$. These higher DA levels found during the night coincide with better performance observed on interval timing - a cognitive task dependent of DA signaling in the dorsal striatum - in the nocturnal phase of the LD cycle ${ }^{50}$. Furthermore, striatal DA and TH levels, as well as DA turnover, also presented daily variations in $\mathrm{LD}$ that did not persist under circadian disruption due to $\mathrm{LL}$ conditions ${ }^{47}$. Indeed, under LD, TH levels were also rhythmic in substantia nigra ( $\mathrm{SN}$ ), the main dopaminergic input to the striatum. Taken together, these results suggest that striatal DA arrythmicity is a consequence of the lack of circadian rhythms in both DA synthesis and degradation. These results led to propose that under LD conditions, rhythmic DA oscillation in the dorsal striatum might be caused by rhythmic input from SN or VTA, as previous studies have demonstrated the expression of circadian clock genes in these structures ${ }^{51,52}$. The protein products of these clock genes act as transcription factors through binding to specific elements in promoter regions, such as E-boxes and RORE elements ${ }^{14}$. As mentioned above, these sequences have been found in the promoter region of components involved in dopaminergic metabolism, such as DAT, TH, and DRD3, suggesting that the expression of these components is under circadian regulation. 
5-HT has also been implied in the circadian regulation of reward related behavior. For example, some findings suggest that cocaine acts in the SCN by enhancing $5-\mathrm{HT}$ signaling ${ }^{53,54}$. On the other hand, serotonin release is rhythmic in nocturnal rodents with a peak during their active phase ${ }^{55}$, and both SERT and sert gene expressions showed rhythmicity in the midbrain ${ }^{56}$. A study using in vivo microdialysis indicated that diurnal changes in the 5-HT metabolite hydroxyindole acetic acid (5HIAA) levels were observed in the PFC but not in the hippocampus, and that the higher levels of 5HIAA might be related to the enhanced neuronal activity of $5-\mathrm{HT}^{57}$. Mouse exposure to chronic stress led to decreased motivation for sucrose reward, while the 5-HT2c receptor antagonist agomelatine was effective in reducing the stress-induced behavior and in increasing perseverance ${ }^{58}$. Taken together, these studies point at 5-HT signaling as a mediator of some aspects of the circadian modulation of reward behavior.

\section{Rhythmic clock gene expression in cortico-limbic areas.}

Many of the core clock genes that drive circadian rhythmicity are expressed in cortico-limbic structures and, in some instances, are rhythmic. For example, the proteins PER1 or PER2 have been reported to oscillate in the NAc, the bed nucleus of the stria terminalis (BNST), the AMG, the Hipp and the PFC ${ }^{39,59-65}$. Furthermore, oscillations of BMAL1 in the NAc has been documented ${ }^{39}$. BMAL1 and PER1 have also been detected in the rat VTA but appear to be constitutively expressed in this region ${ }^{20,39}$. Accordingly, it is possible that the observed rhythmicity in reward may be mediated in part by local NAc clock gene oscillations. Nevertheless, a rhythm in VTA electrical activity has been previously described ${ }^{66}$. Thus, rhythmic activity in these neurons may depend on indirect projections from the $\mathrm{SCN}^{67}$.

Studies conducted in mice carrying mutations in clock genes also suggests an involvement of circadian components in modulating the behavioral responses to rewards. The clock mutant mice (Clock $\Delta 19$ mice) exhibited an increase in the reward value for cocaine and sucrose ${ }^{68}$ and higher excitability of dopamine neurons within the VTA ${ }^{69}$. On the other hand, per1 and per 2 knockout mice presented opposite responses to drug rewards, with the former showing a complete lack of cocaine reward, and the latter a hypersensitized response to cocaine ${ }^{70}$ and increased alcohol consumption ${ }^{71}$. As far as we are concerned, these mutant mice have not been tested with natural rewards, such as food. Considering the differences that natural and non-natural rewards may elicit within the neuronal functioning of the reward circuit ${ }^{5}$, it would be of interest to address this question in the future. Moreover, the use of targeted mutation of clock genes in specific regions relevant to reward processing (for example, the NAc and VTA) may be of great importance in order to elucidate the role of local circadian oscillations in motivated behavioral responses.

The current literature supports the view that rhythmic clock gene expression within cortico-limbic areas of the brain may be responsible for the diurnal variation in motivation behavior towards natural and non-natural rewards.
Although the molecular mechanisms underlying this hypothesis remains to be determined, the dopaminergic system is a plausible candidate to act as an intermerdiary between clock gene rhythmic regulation and timed neuronal activity, especially within the NAc.

\section{POSSIBLE INTERPLAY BETWEEN THE SCN-MASTER CLOCKAND REWARD-RELATED REGIONS.}

The SCN coordinates other secondary oscillators within the central nervous system and peripheral oscillators of the rest of the body - such as the paraventricular nucleus ${ }^{20}$ and the liver ${ }^{72}$ - through neural and humoral outputs. Within the brain, it has been documented that the SCN is an indirect afferent to VTA, where a subpopulation of VTA neurons exhibits a circadian rhythm in impulse activity ${ }^{67}$. This SCN output circuit may be involved in the circadian regulation of motivated behaviors. Besides, direct projection targets of the SCN, including the medial pre-optic area (mPOA) and dorsomedial hypothalamic nucleus $(\mathrm{DMH})$, may modulate reward through indirect neural connections $^{73}$ (Figure 1). Orexinergic neurons in the DMH, for example, encode information about arousal, energy balance, and reward, and project to the $\mathrm{VTA}^{74}$. The dorsal raphe nuclei (DRN) of the midbrain receive direct light input from the circadian visual system and also indirect input from the SCN and are the primary regions containing 5-HT, which is an important mood-related neurotransmitter ${ }^{75}$. In addition, DRN serotonin neurons provide a major input to the $\mathrm{VTA}^{76}$. The lateral habenula $(\mathrm{LHb})$ in the midbrain also receives direct SCN input and is an important inhibitor of dopaminergic activity in the VTA, thus exerting a more robust influence over mood and reward regulation ${ }^{46}$. Indeed, the LHb has been shown to be a self-sustained circadian oscillator within the brain ${ }^{77}$. In this sense, and due to direct projections of the LHb to the VTA, it is possible that the LHb clock modulates (or drives) the rhythmic firing rate observed in DAergic and non-DAergic VTA neurons (reviewed in $^{78}$ ). Furthermore, the LHb innervates the raphe nuclei and may also regulates, in a circadian manner, the 5-HT release to reward-related forebrain regions ${ }^{78}$.

In addition to the central mechanisms underlying the reward circuit, peripheral oscillators may also impact reward. For instance, peripheral hormones like estradiol and cortisol (corticosterone in rodents, both referred as CORT in the present review) modulate neuronal activity, in turn altering circadian activity and reward. Glucocorticoids, which are released when the Hypothalamic-Pituitary-Adrenal (HPA) axis is activated by stress, also play a role in reward across species (reviewed in ${ }^{9}$ ). The HPA axis exhibits both circadian and ultradian rhythms in CORT release ${ }^{79}$. In the Hypothalamic-Nigrostriatal (HNS) axis, the arcuate nucleus (ARC) of the hypothalamus generates neuroendocrine ultradian rhythms closely related to feeding and locomotor behavior ${ }^{80}$. A conceptual model taking into account the interplay between the SCN and these hormonal axes has been described ${ }^{81}$. Hence, it appears that peripheral hormones may play a role in modulating circadian changes in rewardrelated behavior. 


\section{CONCLUSIONS}

Taken together, the current evidence suggests that the circadian modulation of motivation is a robust feature, highlighting the importance of the interaction between the circadian and reward systems. Although more experiments are needed, it is possible that the NAc and/or the VTA act as secondary oscillators and integrate circadian signals from different brain regions to rhythmically modulate motivation and reward-related behaviors (Figure 1). This review also points to a note of caution when interpreting behavioral results of experiments performed under a single time-point.

\section{REFERENCES}

1. Yan J, Wang H, Liu Y, Shao C. Analysis of gene regulatory networks in the mammalian circadian rhythm. PLoS computational biology. 2008;4(10):e1000193. Epub 2008/10/11.

2. Panda S, Hogenesch JB, Kay SA. Circadian rhythms from flies to human. Nature. 2002;417(6886):329-35. Epub 2002/05/17.

3. Harbour VL, Weigl Y, Robinson B, Amir S. Comprehensive mapping of regional expression of the clock protein PERIOD2 in rat forebrain across the 24-h day. PloS one. 2013;8(10):e76391. Epub 2013/10/15.

4. Bailey MR, Jensen G, Taylor K, Mezias C, Williamson C, Silver R, et al. A novel strategy for dissecting goal-directed action and arousal components of motivated behavior with a progressive hold-down task. Behavioral neuroscience. 2015;129(3):269-80. Epub 2015/06/02.

5. DiLeone RJ, Taylor JR, Picciotto MR. The drive to eat: comparisons and distinctions between mechanisms of food reward and drug addiction. Nature neuroscience. 2012;15(10):1330-5. Epub 2012/09/26.

6. Lammel S, Lim BK, Malenka RC. Reward and aversion in a heterogeneous midbrain dopamine system. Neuropharmacology. 2014;76 Pt B:351-9. Epub 2013/04/13.

7. McClung CA. Circadian genes, rhythms and the biology of mood disorders. Pharmacology \& therapeutics. 2007;114(2):222-32. Epub 2007/03/31.

8. Logan RW, McClung CA. Rhythms of life: circadian disruption and brain disorders across the lifespan. Nature reviews Neuroscience. 2019;20(1):49-65. Epub 2018/11/22.

9. DePoy LM, McClung CA, Logan RW. Neural Mechanisms of Circadian Regulation of Natural and Drug Reward. Neural plasticity. 2017;2017:5720842. Epub 2018/01/24.

10. Webb IC. Circadian Rhythms and Substance Abuse: Chronobiological Considerations for the Treatment of Addiction. Current psychiatry reports. 2017;19(2):12. Epub 2017/02/12.

11. Freyberg Z, Logan RW. The Intertwined Roles of Circadian Rhythms and Neuronal Metabolism Fueling Drug Reward and Addiction. Current opinion in physiology. 2018;5:80-9. Epub 2019/01/12.

12. Golombek DA, Rosenstein RE. Physiology of circadian entrainment. Physiological reviews. 2010;90(3):1063-102. Epub 2010/07/29.

13. Kim P, Oster H, Lehnert H, Schmid SM, Salamat N, Barclay JL, et al. Coupling the Circadian Clock to Homeostasis: The Role of Period in Timing Physiology. Endocrine reviews. 2019;40(1):66-95. Epub 2018/09/01.

14. Lowrey PL, Takahashi JS. Genetics of circadian rhythms in Mammalian model organisms. Advances in genetics. 2011;74:175-230. Epub 2011/09/20.

15. Preitner N, Damiola F, Lopez-Molina L, Zakany J, Duboule D, Albrecht $\mathrm{U}$, et al. The orphan nuclear receptor REV-ERBalpha controls circadian transcription within the positive limb of the mammalian circadian oscillator. Cell. 2002;110(2):251-60. Epub 2002/08/02.

16. Guillaumond F, Dardente H, Giguere V, Cermakian N. Differential control of Bmal1 circadian transcription by REV-ERB and ROR nuclear receptors. Journal of biological rhythms. 2005;20(5):391-403. Epub 2005/11/04.

17. Dudley CA, Erbel-Sieler C, Estill SJ, Reick M, Franken P, Pitts S, et al. Altered patterns of sleep and behavioral adaptability in NPAS2-deficient mice. Science. 2003;301(5631):379-83. Epub 2003/07/05.

18. Hampp G, Albrecht U. The circadian clock and mood-related behavior. Communicative \& integrative biology. 2008;1(1):1-3. Epub 2009/08/26.

19. Brown AJ, Pendergast JS, Yamazaki S. Peripheral Circadian Oscillators. The Yale journal of biology and medicine. 2019;92(2):327-35. Epub 2019/06/30.

20. Abe M, Herzog ED, Yamazaki S, Straume M, Tei H, Sakaki Y, et al. Circadian rhythms in isolated brain regions. The Journal of neuroscience: the official journal of the Society for Neuroscience. 2002;22(1):350-6. Epub 2002/01/05.
21. Herzog ED. Neurons and networks in daily rhythms. Nature reviews Neuroscience. 2007;8(10):790-802. Epub 2007/09/21.

22. Mendoza J, Challet E. Brain clocks: from the suprachiasmatic nuclei to a cerebral network. The Neuroscientist : a review journal bringing neurobiology, neurology and psychiatry. 2009;15(5):477-88. Epub 2009/02/20

23. Stephan FK. The "other" circadian system: food as a Zeitgeber. Journal of biological rhythms. 2002;17(4):284-92. Epub 2002/08/08.

24. Tataroglu O, Davidson AJ, Benvenuto LJ, Menaker M. The methamphetamine-sensitive circadian oscillator (MASCO) in mice. Journal of biological rhythms. 2006;21(3):185-94. Epub 2006/05/30.

25. Mistlberger RE. Neurobiology of food anticipatory circadian rhythms. Physiology \& behavior. 2011;104(4):535-45. Epub 2011/04/30.

26. Pendergast JS, Yamazaki S. The Mysterious Food-Entrainable Oscillator: Insights from Mutant and Engineered Mouse Models. Journal of biological rhythms. 2018;33(5):458-74. Epub 2018/07/24.

27. Salamone JD, Correa M. The mysterious motivational functions of mesolimbic dopamine. Neuron. 2012;76(3):470-85. Epub 2012/11/13.

28. Verharen JPH, Adan RAH, Vanderschuren L. How Reward and Aversion Shape Motivation and Decision Making: A Computational Account. The Neuroscientist : a review journal bringing neurobiology, neurology and psychiatry. 2020;26(1):87-99. Epub 2019/03/15.

29. Berridge KC, Robinson TE. What is the role of dopamine in reward: hedonic impact, reward learning, or incentive salience? Brain research Brain research reviews. 1998;28(3):309-69. Epub 1998/12/22.

30. Berridge KC, Robinson TE, Aldridge JW. Dissecting components of reward: 'liking', 'wanting', and learning. Current opinion in pharmacology. 2009;9(1):65-73. Epub 2009/01/24.

31. Yagishita S. Transient and sustained effects of dopamine and serotonin signaling in motivation-related behavior. Psychiatry and clinical neurosciences. 2020;74(2):91-8. Epub 2019/10/11.

32. Feillet CA, Bainier C, Mateo M, Blancas-Velazquez A, Salaberry NL, Ripperger JA, et al. Rev-erbalpha modulates the hypothalamic orexinergic system to influence pleasurable feeding behaviour in mice. Addiction biology. 2017;22(2):411-22. Epub 2015/12/04.

33. Kelley AE. Ventral striatal control of appetitive motivation: role in ingestive behavior and reward-related learning. Neuroscience and biobehavioral reviews. 2004;27(8):765-76. Epub 2004/03/17.

34. Sanders AC, Hussain AJ, Hen R, Zhuang X. Chronic blockade or constitutive deletion of the serotonin transporter reduces operant responding for food reward. Neuropsychopharmacology: official publication of the American College of Neuropsychopharmacology. 2007;32(11):2321-9. Epub 2007/03/16.

35. Miyazaki K, Miyazaki KW, Doya K. Activation of dorsal raphe serotonin neurons underlies waiting for delayed rewards. The Journal of neuroscience : the official journal of the Society for Neuroscience. 2011;31(2):469-79. Epub 2011/01/14.

36. Miyazaki KW, Miyazaki K, Tanaka KF, Yamanaka A, Takahashi A, Tabuchi S, et al. Optogenetic activation of dorsal raphe serotonin neurons enhances patience for future rewards. Current biology : CB. 2014;24(17):2033-40. Epub 2014/08/27.

37. Li Y, Zhong W, Wang D, Feng Q, Liu Z, Zhou J, et al. Serotonin neurons in the dorsal raphe nucleus encode reward signals. Nature communications. 2016;7:10503. Epub 2016/01/29.

38. Albrecht U. The circadian clock, reward, and memory. Frontiers in molecular neuroscience. 2011;4:41. Epub 2011/11/16.

39. Webb IC, Baltazar RM, Wang X, Pitchers KK, Coolen LM, Lehman MN. Diurnal variations in natural and drug reward, mesolimbic tyrosine hydroxylase, and clock gene expression in the male rat. Journal of biological rhythms. 2009;24(6):465-76. Epub 2009/11/21.

40. Bainier C, Mateo M, Felder-Schmittbuhl MP, Mendoza J. Circadian rhythms of hedonic drinking behavior in mice. Neuroscience. 2017;349:229-38. Epub 2017/03/14.

41. Acosta J, Bussi IL, Esquivel M, Hocht C, Golombek DA, Agostino PV. Circadian modulation of motivation in mice. Behavioural brain research. 2020;382:112471. Epub 2020/01/21.

42. Yoon SO, Chikaraishi DM. Tissue-specific transcription of the rat tyrosine hydroxylase gene requires synergy between an AP-1 motif and an overlapping E box-containing dyad. Neuron. 1992;9(1):55-67. Epub 1992/07/01

43. Kawarai T, Kawakami H, Yamamura Y, Nakamura S. Structure and organization of the gene encoding human dopamine transporter. Gene. 1997;195(1):11-8. Epub 1997/08/11.

44. Ozburn AR, Falcon E, Twaddle A, Nugent AL, Gillman AG, Spencer SM, et al. Direct regulation of diurnal Drd3 expression and cocaine reward by NPAS2. Biological psychiatry. 2015;77(5):425-33. Epub 2014/12/03. 
45. Sleipness EP, Sorg BA, Jansen HT. Diurnal differences in dopamine transporter and tyrosine hydroxylase levels in rat brain: dependence on the suprachiasmatic nucleus. Brain research. 2007;1129(1):34-42. Epub 2006/12/13.

46. Parekh PK, McClung CA. Circadian Mechanisms Underlying RewardRelated Neurophysiology and Synaptic Plasticity. Frontiers in psychiatry. 2015;6:187. Epub 2016/01/23.

47. Bussi IL, Levin G, Golombek DA, Agostino PV. Involvement of dopamine signaling in the circadian modulation of interval timing. The European journal of neuroscience. 2014;40(1):2299-310. Epub 2014/04/03.

48. Castaneda TR, de Prado BM, Prieto D, Mora F. Circadian rhythms of dopamine, glutamate and GABA in the striatum and nucleus accumbens of the awake rat: modulation by light. Journal of pineal research. 2004;36(3):177-85. Epub 2004/03/11.

49. Hood S, Cassidy P, Cossette MP, Weigl Y, Verwey M, Robinson B, et al. Endogenous dopamine regulates the rhythm of expression of the clock protein PER2 in the rat dorsal striatum via daily activation of D2 dopamine receptors. The Journal of neuroscience : the official journal of the Society for Neuroscience. 2010;30(42):14046-58. Epub 2010/10/22.

50. Agostino PV, do Nascimento M, Bussi IL, Eguia MC, Golombek DA. Circadian modulation of interval timing in mice. Brain research. 2011;1370:154-63. Epub 2010/11/17.

51. Li SX, Liu LJ, Jiang WG, Lu L. Morphine withdrawal produces circadian rhythm alterations of clock genes in mesolimbic brain areas and peripheral blood mononuclear cells in rats. Journal of neurochemistry. 2009;109(6):1668-79. Epub 2009/04/23.

52. Natsubori A, Honma K, Honma S. Differential responses of circadian Per2 expression rhythms in discrete brain areas to daily injection of methamphetamine and restricted feeding in rats. The European journal of neuroscience. 2013;37(2):251-8. Epub 2012/10/31.

53. Glass JD, Brager AJ, Stowie AC, Prosser RA. Cocaine modulates pathways for photic and nonphotic entrainment of the mammalian SCN circadian clock. American journal of physiology Regulatory, integrative and comparative physiology. 2012;302(6):R740-50. Epub 2012/01/06.

54. Prosser RA, Stowie A, Amicarelli M, Nackenoff AG, Blakely RD, Glass JD. Cocaine modulates mammalian circadian clock timing by decreasing serotonin transport in the SCN. Neuroscience. 2014;275:184-93. Epub 2014/06/21.

55. Dudley TE, DiNardo LA, Glass JD. Endogenous regulation of serotonin release in the hamster suprachiasmatic nucleus. The Journal of neuroscience : the official journal of the Society for Neuroscience. 1998;18(13):5045-52. Epub 1998/06/23.

56. Ushijima K, Koyanagi S, Sato Y, Ogata T, Matsunaga N, Fujimura A, et al. Role of activating transcription factor-4 in 24-hour rhythm of serotonin transporter expression in the mouse midbrain. Molecular pharmacology. 2012;82(2):264-70. Epub 2012/05/11.

57. Nakayama K. Diurnal rhythmin extracellularlevels of 5-hydroxyindoleacetic acid in the medial prefrontal cortex of freely moving rats: an in vivo microdialysis study. Progress in neuro-psychopharmacology \& biological psychiatry. 2002;26(7-8):1383-8. Epub 2002/12/28.

58. Bergamini G, Cathomas F, Auer S, Sigrist H, Seifritz E, Patterson M, et al. Mouse psychosocial stress reduces motivation and cognitive function in operant reward tests: A model for reward pathology with effects of agomelatine. European neuropsychopharmacology : the journal of the European College of Neuropsychopharmacology. 2016;26(9):1448-64. Epub 2016/07/17.

59. Amir S, Lamont EW, Robinson B, Stewart J. A circadian rhythm in the expression of PERIOD2 protein reveals a novel SCN-controlled oscillator in the oval nucleus of the bed nucleus of the stria terminalis. The Journal of neuroscience : the official journal of the Society for Neuroscience. 2004;24(4):781-90. Epub 2004/01/30.

60. Lamont EW, Robinson B, Stewart J, Amir S. The central and basolateral nuclei of the amygdala exhibit opposite diurnal rhythms of expression of the clock protein Period2. Proceedings of the National Academy of Sciences of the United States of America. 2005;102(11):4180-4. Epub 2005/03/05.

61. Perrin JS, Segall LA, Harbour VL, Woodside B, Amir S. The expression of the clock protein PER2 in the limbic forebrain is modulated by the estrous cycle. Proceedings of the National Academy of Sciences of the United States of America. 2006;103(14):5591-6. Epub 2006/03/24.
62. Angeles-Castellanos M, Mendoza J, Escobar C. Restricted feeding schedules phase shift daily rhythms of c-Fos and protein Per1 immunoreactivity in corticolimbic regions in rats. Neuroscience. 2007;144(1):344-55. Epub 2006/10/19.

63. Verwey M, Khoja Z, Stewart J, Amir S. Differential regulation of the expression of Period 2 protein in the limbic forebrain and dorsomedial hypothalamus by daily limited access to highly palatable food in fooddeprived and free-fed rats. Neuroscience. 2007;147(2):277-85. Epub 2007/06/05.

64. Waddington Lamont E, Harbour VL, Barry-Shaw J, Renteria Diaz L, Robinson B, Stewart J, et al. Restricted access to food, but not sucrose, saccharine, or salt, synchronizes the expression of Period 2 protein in the limbic forebrain. Neuroscience. 2007;144(2):402-11. Epub 2006/10/28.

65. Feillet CA, Mendoza J, Albrecht U, Pevet P, Challet E. Forebrain oscillators ticking with different clock hands. Molecular and cellular neurosciences. 2008;37(2):209-21. Epub 2007/11/13.

66. Luo AH, Georges FE, Aston-Jones GS. Novel neurons in ventral tegmental area fire selectively during the active phase of the diurnal cycle. The European journal of neuroscience. 2008;27(2):408-22. Epub 2008/01/25.

67. Luo AH, Aston-Jones G. Circuit projection from suprachiasmatic nucleus to ventral tegmental area: a novel circadian output pathway. The European journal of neuroscience. 2009;29(4):748-60. Epub 2009/02/10.

68. Roybal K, Theobold D, Graham A, DiNieri JA, Russo SJ, Krishnan V, et al. Mania-like behavior induced by disruption of CLOCK. Proceedings of the National Academy of Sciences of the United States of America. 2007;104(15):6406-11. Epub 2007/03/24.

69. McClung CA, Sidiropoulou K, Vitaterna M, Takahashi JS, White FJ, Cooper DC, et al. Regulation of dopaminergic transmission and cocaine reward by the Clock gene. Proceedings of the National Academy of Sciences of the United States of America. 2005;102(26):9377-81. Epub 2005/06/22.

70. Abarca C, Albrecht U, Spanagel R. Cocaine sensitization and reward are under the influence of circadian genes and rhythm. Proceedings of the National Academy of Sciences of the United States of America. 2002;99(13):9026-30. Epub 2002/06/27.

71. Spanagel R, Pendyala G, Abarca C, Zghoul T, Sanchis-Segura C, Magnone MC, et al. The clock gene Per2 influences the glutamatergic system and modulates alcohol consumption. Nature medicine. 2005;11(1):35-42. Epub 2004/12/21.

72. Stokkan KA, Yamazaki S, Tei H, Sakaki Y, Menaker M. Entrainment of the circadian clock in the liver by feeding. Science. 2001;291(5503):490-3. Epub 2001/02/13.

73. Mendoza J, Challet E. Circadian insights into dopamine mechanisms. Neuroscience. 2014;282:230-42. Epub 2014/10/05.

74. Aston-Jones G, Smith RJ, Moorman DE, Richardson KA. Role of lateral hypothalamic orexin neurons in reward processing and addiction. Neuropharmacology. 2009;56 Suppl 1:112-21. Epub 2008/07/29.

75. Morin LP. Neuroanatomy of the extended circadian rhythm system. Experimental neurology. 2013;243:4-20. Epub 2012/07/07.

76. Wang HL, Zhang S, Qi J, Wang H, Cachope R, Mejias-Aponte CA, et al. Dorsal Raphe Dual Serotonin-Glutamate Neurons Drive Reward by Establishing Excitatory Synapses on VTA Mesoaccumbens Dopamine Neurons. Cell reports. 2019;26(5):1128-42 e7. Epub 2019/01/31.

77. Guilding C, Hughes AT, Piggins HD. Circadian oscillators in the epithalamus. Neuroscience. 2010;169(4):1630-9. Epub 2010/06/16.

78. Mendoza J. Circadian neurons in the lateral habenula: Clocking motivated behaviors. Pharmacology, biochemistry, and behavior. 2017;162:55-61. Epub 2017/07/02.

79. Lightman S. Rhythms Within Rhythms: The Importance of Oscillations for Glucocorticoid Hormones. In: Sassone-Corsi P, Christen Y, editors. A Time for Metabolism and Hormones. Cham (CH)2016. p. 87-99.

80. Prendergast BJ, Zucker I. Ultradian rhythms in mammalian physiology and behavior. Current opinion in neurobiology. 2016;40:150-4. Epub 2016/08/30.

81. Laje R, Agostino PV, Golombek DA. The Times of Our Lives: Interaction Among Different Biological Periodicities. Frontiers in integrative neuroscience. 2018;12:10. Epub 2018/03/30.

82. Agostino PV, Acosta, J., \& Meck, W. H. Neurobiology of Circadian and Interval Timing. eLS. 2017:1-11. 


\section{From the ruler to the smartphone tasks applied to identify sleep deprivation}

Giannina J. Bellone $e^{1,2,3^{*}}$ Malena Mul Fedele ${ }^{1}$ Carolina Abulafia 1,4 Santiago A. Plano ${ }^{1,2}$ Diego A. Golombek ${ }^{2}$ Daniel E. Vigo ${ }^{1,5}$

${ }^{1}$ Institute for Biomedical Research, Catholic University of Argentina (UCA) - National Scientific and Technical Research Council (CONICET).

${ }^{2}$ Chronobiology Lab, National University of Quilmes (UNQ) and National Scientific and Technical Research Council (CONICET).

${ }^{3}$ Faculty of Psychology, Catholic University of Argentina (UCA).

${ }^{4}$ Fundación para la Lucha contra las Enfermedades Neurológicas de la Infancia (FLENI).

${ }^{5}$ Faculty of Psychology and Educational Sciences, KU Leuven.

\begin{abstract}
Sleep-related health disorders and fatigue represent a major public health issue worldwide. It is estimated that $13 \%$ of work-related injuries are attributed to sleep problems. Consequences of sleep deprivation and fatigue not only impact the person, but also can have social impact. Numerous objective tests have been designed to assess whether a person is properly rested to safely carry out his/her job. These techniques are focused mainly on the measurement of response time, alertness and sustained attention proxy for sleep deprivation and fatigue, and they should be properly validated. Over the last 35 years, the Psychomotor Vigilance Test has been the gold standard widely used because it can be performed in different environments and due it operational characteristics. However, validity procedures do not report detailed information about specificity, sensitivity, negative and positive predictive value. These data are essential to determine if the test is optimal for it implementation. The purpose of this review is to go over the evolution of the techniques applied to identify sleep deprivation, starting from the basic and analog reaction test to the most current portable and digital techniques.
\end{abstract}

Keywords: PVT; Sleep Deprivation; Alertness; Fatigue; Performance; Validity
Corresponding author:

Giannina J. Bellone

E-mail: giannina.bellone@gmail.com 


\section{INTRODUCTION}

Sleep-related health disorders and fatigue represent a major public health issue worldwide ${ }^{1,2}$. After an extensive analysis on a complete description of fatigue, Phillips $(2015)^{3}$ defines fatigue as: "A suboptimal psychophysiological condition caused by exertion [...]. The context of exertion is described by the value and meaning of performance to the individual; rest and sleep history; circadian effects; psychosocial factors spanning work and home life; individual traits; diet; health, fitness and other individual states; and environmental conditions. The fatigue condition results in changes in strategies or resource use such that original levels of mental processing or physical activity are maintained or reduced." Insufficient sleep has a negative impact on different aspects of wakefulness ${ }^{4}$.

It is estimated that $13 \%$ of work-related injuries are attributed to sleep problems, and workers with sleep problems had a 1.62 times higher risk of being injured than workers without them $^{5}$. In the road safety field, effects of sleep deprivation are responsible for approximately $20 \%$ of all serious motor vehicle accidents and up to $43 \%$ of commuter train accidents are thought to be due to this problem ${ }^{2}$.

In the last 50 years, numerous objective tests have been designed to assess whether a person is properly rested to safely carry out his/her job. These tests have been included in occupational or military safety protocols in order to avoid accidents or catastrophes related to fatigue ${ }^{6}$.

Objective tests designed to assess whether a worker is well rested have focused mainly on the measurement of: response time (the minimal time needed to respond to a stimulus), alertness (a cognitive capacity characterized by being fully aware of the self and the surroundings), and attention, specifically sustained attention (the ability to keep the focus of attention on a task or event for a prolonged period of time $)^{7-9}$.

Many of the techniques used to measure response time, alertness and sustained attention, as a proxy for sleep deprivation and fatigue, have been widely used and thoroughly validated in experimental and clinical environments. As sleep-related health disorders and fatigue are increasing worldwide and generating occupational and social impact, it is imperative to perform these tests in operational environments to effectively predict sleep deprivation and prevent incidents and accidents ${ }^{10,11}$. Rapidly growing technological developments are constantly producing readily available commercial devices that are increasingly portable and easily implemented in the operational environment; however, it is imperative that these new devices are valid for their application ${ }^{12}$ ).

The purpose of this non-systematic review is to go over the evolution of the techniques applied to identify sleep deprivation, starting from the basic and analog reaction test to the most current portable and digital techniques.

\section{THEORETICAL BACKGROUND}

Alertness is the state of a healthy, well-rested person that allows an individual to sustain his/her attention and give a timely response to any situation ${ }^{13}$. Lack of alertness is one of the most specific symptoms of fatigue or sleep deprivation ${ }^{8}$.
Banks et al. (2019) define fatigue as the inability to function at the optimal level, because the physical and mental effort (of all waking activities, not just work) exceeds the existing capacity ${ }^{14}$. The level of fatigue depends on the task performed by the individual (for example, for pilots, their ability to safely operate an aircraft; for cabin crew, their ability to perform safety-related duties) ${ }^{4}$.

Sleep deprivation (partial or total) leads to neurobehavioral consequences. Some of them are: degradation of alertness, slower reaction time, decreased vigilance, lapsing, slower problemsolving and reasoning abilities, impaired accuracy and decisionmaking skills, increased omission and commission errors, reduced psychomotor skills ${ }^{7,8,15,16}$. For example, when sleep is limited to 4-6 hours per night, effects on cognitive performance occur, producing a progressive cognitive dysfunction ${ }^{12,17,18}$.

An adequate performance refers to achievement of an optimal and effective functioning of cognitive abilities and executive functions like psychomotor vigilance, alertness, memory, reasoning or decision-making among others ${ }^{13}$. One night of sleep deprivation can generate more damage on health than sleep throughout several nights, generating detrimental effects on performance ${ }^{17}$. Cognitive impairment can be measured within the first several minutes of performance, especially on a boring monotonous task ${ }^{7,19}$.

The effects of sleep deprivation on performance are comparable with the effects produced by alcohol consumption; 17 hours awake is equivalent to a blood alcohol content of 0.05 , the legal limit for driving in most countries ${ }^{20}$. Specifically, similarities have been observed in the reduction of psychomotor skills ${ }^{15}$. This similarity in effects between fatigue and alcohol highlights the need to develop and implement tools for the detection of sleep deprivation.

The degradation of performance represents the most dangerous effect of sleep loss in the workplace, increasing the possibility of near-accidents and accidents in workers, especially in those with non-traditional shifts (nocturnal or shift workers), which include drivers of professional vehicles, train operators or health personnel ${ }^{7,15,18}$. Shift work includes working evenings, nights, or rotating shifts and is often associated with shorter and disrupted sleep periods ${ }^{21}$. Surveys, observational data, and anecdotal incident reports reveal that shift workers often experience sleep episodes ${ }^{18}$ and they frequently complain about excessive daytime sleepiness. ${ }^{10}$

Therefore, consequences of sleep deprivation and fatigue not only impact the person, but also can have social consequences such as industrial catastrophes, medical errors, transportation accidents, and security breaches ${ }^{22}$. The relationship between work accidents and the decrease in operational productivity caused by drowsiness or fatigue is difficult to measure ${ }^{6}$, yet the effects of sleep deprivation must be studied and quantified to take preventive actions?

Subjective evaluation tools like sleep and alertness measurement techniques can provide useful clinical information. However, the lack of sensitivity and specificity make these techniques subject to many influences which can mask the real information ${ }^{13}$. 
This could be due to unintended bias, motivational factors, demands inherent to the experiment, distractions by environmental stimulation, stress, food intake, posture and activity, room temperature, lighting conditions, drug intake and even purposeful falsification, among others. Also, it has been shown that sleepy subjects cannot reliably self-assess their impairment when they are in a state of drowsiness ${ }^{10,13,23}$. The multiple limitations of subjective techniques highlight the dire need for brief, validated and objective measures to evaluate fatigue?

A fitness for duty test is optimal when it measures with criterion validity for both risk factors (like fatigue) and job performance. It must have certain characteristics: be valid (measure what it intends to measure as a fatigue-sensitive behavior), reliable (measure the same consistently), specific (minimizing false alarms), generalizable (for all users, taking into account individual differences) and sensitive (predicting unacceptable fatigue levels and minimizing lost events) $)^{12,24}$.

It is essential for that test to have the same sensitivity as the laboratory test and to be feasible to apply in the workplace and comply with scientific and operational properties. In particular, it should be easy to use, portable, brief, without effects due to practice or learning and the obtained results must be readily available ${ }^{24}$. These tests must provide feedback to operator about his/her alertness level, that is, if the subject being assessed is able to perform a given task. ${ }^{18,24-26}$.

Reaction Time tests, in which subjects simply respond as fast as possible to a stimulus, are sensitive to asses sleep deprivation because they can evaluate changes in the alertness state caused by inadequate sleep, and have proved to be useful to understand the effects of sleep deprivation", 26-28. Woods et all (2015) define the Simple Reaction Time (SRT) as the minimal time needed to respond to a stimulus ${ }^{29}$. A slow response time affects performance, for example by increasing risk for accidental falls and motor vehicle accidents ${ }^{11}$.

Sleep deprivation leads to a general slowing of response times. Restricting sleep to 6 hours throughout few nights leads to a slower response time during the day ${ }^{12}$. The same level of sleep restriction sustained for two weeks generates a degree of impairment comparable to two nights of total sleep deprivation $(\mathrm{TSD})^{17}$; and chronic partial sleep deprivation (CPSL) of approximately 5 hours every night results in decreased performance which can lead to accidents ${ }^{21}$. Even more so, chronic sleep deprivation of 4 hours per night may generate a continuous performance impairment and would most likely lead to personal or work-related accidents ${ }^{17,7-8}$.

Jafe et al. (2018) ${ }^{28}$ emphasize the value of studies focused on performance metrics - such as reaction time - for the understanding of the specific effects sleep duration and sleep deprivation.

\section{RELEVANT TECHNIQUES FOR MEASURING REACTION TIME}

The Ruler Drop Method (RDM): It consist in grab a ruler when someone else throws it at an unexpected moment. The falling ruler is the stimulus and grabbing it is the motor (voluntary) response. Shorter the time, the faster is the reaction ${ }^{30}$.
This is one of the first analog method to assess reaction time and has also been field-expedient and widely accepted as a valid method for testing reaction time. Aranha et al. (2017), developed a study in school going children comparing the RDM to a mobile-based software application and found that the RDM is a moderate to good method for determining reaction time ${ }^{28}$. It has been used in clinical environment for testing speed visual reaction time in people with and without diabetes ${ }^{30}$.

RTclin (University of Michigan, USA, 2010): A similar method to the RDM is the RTclin, a clinical reaction time apparatus designed to emulate a ruler, that measures reaction time. It quantifies the time required to catch a suspended vertical shaft by pinch grip released at random intervals. The subject has to open his hands and catch the device as soon as he perceives it being released and grasps it quickly as possible ${ }^{28}$. It has been used in experimental environments. A study in which 65 healthy adults performed clinical and computerized reaction time tasks (RTclin and RTcomp) under simple and dual-task conditions confirmed that RTclin is a reliable and valid measure of reaction time $^{31}$. Further work is needed before recognition RTclin can be applied in the clinical setting ${ }^{11}$.

The Auditory Vigilance Task (Wilkinson, 1970): The stimulus in this task is an auditory tone ${ }^{32}$. The original task, is a one-hour auditory technique in which subjects must listen to spaced tones of $500 \mathrm{~ms}$ of duration every two seconds ${ }^{25}$. In a shorter duration task (10 minutes), the auditory tone is turned on for $475 \mathrm{~ms}$ and off for $48 \mathrm{~ms}$ If no response occurred the counter was reset after $30000 \mathrm{~ms}^{32}$. During the Auditory Vigilance Task, subjects have to look at a paper located on the computer screen and no response time feedback is given. The response box had double pole double throw buttons which gave two electrical outputs when pressed: one to the computer to stop the stimulus and one to the digital recorder. It has been demonstrated that the $1 \mathrm{~h}$ Wilkinson Auditory Vigilance Task is sensitive to one night of sleep deprivation ${ }^{25}$. The 10 minutes Auditory Vigilance Task is sensitive to sleep deprivation, to performance at an adverse circadian phase, and to time on task decrements ${ }^{32}$.

The four choice portable cassette recording apparatus (Wilkinson \& Houghton, 1975): It is the reaction time task of choice (10 minutes of duration). It consists of four lights arranged in a square and four keys arranged in a similar way. When one of the four lights comes on, the subject must press the button that corresponds geometrically to the activated light. The light goes out and after $120 \mathrm{~ms}$ any of the four lights come on again, independently of the response. The cycle is repeated in a randomly fashion ${ }^{25}$.

Wilkinson Simple Visual Reaction Time (VRT) Task (KE Developments, Ltd., Cambridge, England, 1982): It is a ten-minute auditory task carried out with a portable cassette recording device and a modified tape. It initiates with a visual stimulus, and a four digits clock in milliseconds visually displayed. The subject has to press a microswich that stops the burst of tones on the audio tape and the digital clock, allowing the subject 1.5 seconds to read the value. 
The start of the stimulus of the next series of numbers occurs randomly between 1 and 10 seconds after the previous response ${ }^{26}$.

It was found that the Wilkinson VRT Task and The four choice portable cassette recording apparatus are particularly sensitive to the effects of sleep deprivation after only five minutes of testing ${ }^{25}$. Performance on Wilkinson VRT Task was sensitive to as little as one night without sleep ${ }^{25,26}$.

Occupational safety performance assessment test (OSPAT) (Romteck, Western Australia, 1998): The test consists in a software task with a duration of 60 seconds. It measures reaction time, sustained attention and motor coordination. ${ }^{33}$ OSPAT has been validated for sleep deprivation and it shows to be sensitive to the detection of TSD from a single night (24 hours of prolonged wakefulness ${ }^{33}$.

\section{The gold standard: Psychomotor Vigilance Task}

The PVT is one of the most sensitive measures of performance impairment by sleep deprivation ${ }^{22}$. It consists in a visual simple reaction time test based in a sustained attention task for fatigue detection ${ }^{7,12,34}$. The objective of the PVT is to motor response as quickly as possible by clicking a button to the visual stimuli presented on a screen with a random inter-trial interval. ${ }^{16,35}$. Average reaction time in PVT increases in length overall after a period of sleep deprivation and is associated with eye closure and micro-sleep ${ }^{7,36}$. It has proven to be a valid test to measure alert reduction as a result of PSD or TSD ${ }^{34,37,38}$.

Over the last 35 years, the Psychomotor Vigilance Test has been the gold standard widely used because of its advantages over other tests ${ }^{24,37,39}$. Some of the most important advantages are that: it can be used not only in experimental and clinical studies, but also in operational environments ${ }^{35-37}$; it is useful for repeated use in within-subject designs ${ }^{35}$; It is a brief test, different versions of PVT can last from 2 minutes to 10 minutes ${ }^{35,36,40}$; and it can measure and estimate differences in the aptitude between different subjects ${ }^{35}$.
The most used outcomes that the PVT give back are: mean Response Time (RT) (which are valid if they are $\geq 100 \mathrm{~ms}$ and $\leq 500 \mathrm{~ms}$ ); false starts (when the RTs are less than $100 \mathrm{~ms}$ ) and lapses (reaction times greater than $500 \mathrm{~ms}$ ), ${ }^{97,39}$. Some others outcomes are: mean1/RT; fastest 10\% RT; fastest $10 \%$ 1/RT; median RT; slowest 10\% RT; slowest 10\% 1/RT; lapse probability and other particular outcomes.

PVT-192 (Ambulatory Monitoring Inc., Model PVT-192): The first PVT (Ambulatory Monitoring Inc., Model PVT-192) was designed by Dinges and Powell in 1984 as an evolution of the Wilkinson Visual Reaction Test ${ }^{39,41}$. It is a hand-held, self-contained system which consists in a simple reaction time task of 10 minutes of duration and inter-stimulus interval (ISI) that ranges between 2 and 10 seconds ${ }^{16,40}$. The stimulus is composed of a counter that has four digits and can be seen on the screen. To answer the subject has to press the button when the stimulus is received. Each RT is stored on the device and then loaded on a $\mathrm{PC}^{42}$. Inter-stimulus intervals, time on task and ISI parameterization represent the "vigilance" aspect of the PVT".

It has been demonstrated that the 10min PVT version is valid and highly sensitive to the effects of sleep deprivation (TSD and CPSD) and fatigue because the performance deteriorates faster in sleep deprived than in alert subjects with time on $\operatorname{task}^{7,9,26,43}$. This can be seen in the higher RT, number of lapses, mean RT, inverse of the mean RT, the mean of the $10 \%$ of the fastest and slowest RT, and in the increase of omission errors and commission errors $8,9,44$.

In recent years, shorter variants of the PVT have been developed which have been useful to measure the decrease in performance due to sleep deprivation ${ }^{8}$. Some of these variants are described below:

PVT-A (Basner \& Dinges, 2012): This computer version of the PVT has an average duration of 6.5 minutes, which makes it more feasible to use it in operating and clinical settings. ${ }^{21}$

Table 1. Relevant techniques for reaction time and sleep deprivation detection

\begin{tabular}{|c|c|c|c|c|c|c|c|c|c|}
\hline Task/ Test & Author & Year & Country & Device & Measure & Duration & Enviroment & Validity & Objective \\
\hline The Ruler Drop Method & Unknown & $\mathrm{n} / \mathrm{s}$ & $\mathrm{n} / \mathrm{s}$ & Ruler & Reaction Time & Random & $\begin{array}{c}\text { Experimental \& Clinical: } \\
\text { school going children/ } \\
\text { Diabetes and non- } \\
\text { diabetes patients }\end{array}$ & No & $\begin{array}{l}\text { Reaction time. } \\
\text { Speed visual } \\
\text { reaction }\end{array}$ \\
\hline RTclin & $\begin{array}{l}\text { University of } \\
\text { Michigan }\end{array}$ & 2010 & USA & $\begin{array}{l}\text { Shaft and } \\
\text { Pinch Grip }\end{array}$ & Reaction Time & Random & $\begin{array}{l}\text { Experimental \& Sport } \\
\text { related }\end{array}$ & Yes & Reaction time \\
\hline Auditory Vigilance Task & Wilkinson & 1970 & England & $\begin{array}{l}\text { Auditory } \\
\text { technique }\end{array}$ & Reaction Time & $1 \mathrm{~h}$ & Experimental & Yes & $\begin{array}{c}\text { Sleep } \\
\text { deprivation }\end{array}$ \\
\hline $\begin{array}{l}\text { The four choice } \\
\text { portable cassette } \\
\text { recording apparatus }\end{array}$ & $\begin{array}{l}\text { Wilkinson and } \\
\text { Houghton }\end{array}$ & 1975 & England & $\begin{array}{l}\text { Lights and } \\
\text { buttons }\end{array}$ & Reaction Time & $10 \mathrm{~m}$ & Experimental & Yes & $\begin{array}{c}\text { Sleep } \\
\text { deprivation }\end{array}$ \\
\hline $\begin{array}{l}\text { Wilkinson Simple } \\
\text { Visual Reaction Time } \\
\text { (VRT) Task }\end{array}$ & Wilkinson & 1982 & England & $\begin{array}{l}\text { Cassette } \\
\text { recording } \\
\text { device and } \\
\text { microswitch }\end{array}$ & Reaction Time & $10 \mathrm{~m}$ & Experimental & Yes & $\begin{array}{c}\text { Sleep } \\
\text { deprivation }\end{array}$ \\
\hline $\begin{array}{l}\text { Occupational } \\
\text { safety performance } \\
\text { assessment test }\end{array}$ & Romteck & 1998 & Australia & $\begin{array}{c}\text { Portable } \\
\text { device }\end{array}$ & $\begin{array}{l}\text { Reaction Time; } \\
\text { sustained } \\
\text { attention, } \\
\text { motor } \\
\text { coordination }\end{array}$ & 60 seconds & Sleep deprivation & Yes & $\begin{array}{l}\text { Total sleep } \\
\text { deprivation }\end{array}$ \\
\hline
\end{tabular}


Looking at a computer screen, subjects should respond as quickly as possible to a yellow counter on it; which then shows the response time for 1s. The PVT-A has been validated in experimental environment demonstrating to be highly accurate, sensitive and specific ${ }^{16,21}$.

PC-PVT (Khitrov, 2013): In order to improve the traditional PVT interface, a version of PVT for PC, which runs in Windows 7 operating system, was developed consisting of two separate programs, an administrator and a tester. The tester consists of a PVT session that can last from 5 to 10 minutes and uses a five-digit millisecond counter as a stimulus. The answer is granted with the click of the mouse and, after each response, the RT is displayed on the screen for $500 \mathrm{~ms}$. The PC-PVT has been validated as a technique for measuring neurobehavioral performance and as a sleep deprivation detection ${ }^{42}$.

The emergence of new technologies allowed the development of more portable PVT. This type of devices facilitatesthe use of this technique in operational environments of remote conditions ${ }^{44}$.

PalmPVT (Walter Reed Army Institute of Research, 2005): This version of the PVT was developed and validated on personal data assistant devices (PDAs) and runs on a Palm-Os. In a 5-minute duration task, the stimuli are presented on the device screen and the subject must respond on the same screen by pressing a specific button ${ }^{41}$. This version responds as a valid and reliable device for measuring sleep deprivation and fatigue. Regarding results are comparable to the longer values of the PVT-192 despite the different stimulus characteristics ${ }^{8,41}$.

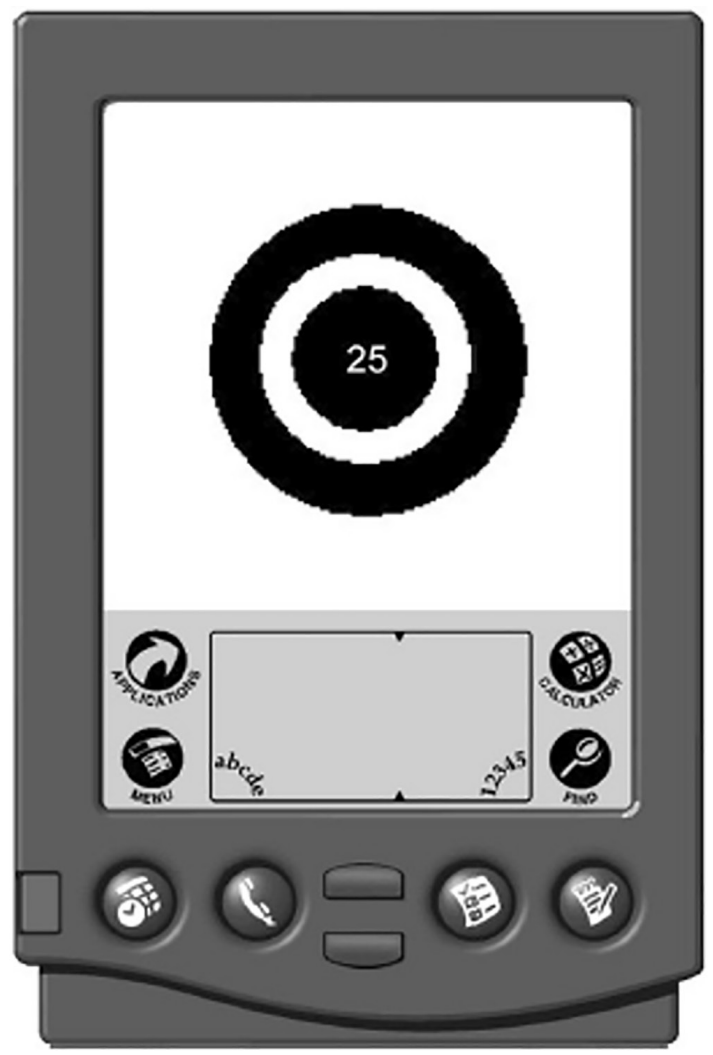

Figure 1. PalmPVT (Walter Reed Army Institute of Research, 2005). Subjects should look at a screen and press a button as soon as the counter stimulus appears.
PVT-B (Basner et. al, 2011): This is the first version of the 10-minute PVT in a 3-minute mode. As in the 10-min version, subjects should look at a screen and press a button as soon as the counter stimulus appears, which stops the counter and the response time can be observed ${ }^{45}$. It was validated in controlled laboratory studies and had demonstrated to be less sensitive to the detection of sleep deprivation due to its short duration ${ }^{45}$. Likewise, the PVT-B grants faster response times, more false starts and less lapses than the traditional PVT performance. Although in general the tool is sensitive and specific for detecting sleep deprivation, especially in environments where it is not possible to run longer tests, a validation process is required in this type of environment ${ }^{19,45}$.

Fitness For Duty (FFD)-PVT (Basner \& Rubinstein, 2011): In this 3-minute version, the signal speed is increased (interval between stimuli from 1 to 4 seconds) and the definition of lapse is reduced from the standard definition of $\geq 500 \mathrm{~ms}$ to $\geq 355 \mathrm{~ms}$. It was validated against the standard 10-minute PVT in total and partial sleep deprivation paradigms, and it was shown that it reaches similar levels of sensitivity and specificity. It was able to predict performance

on a simulated luggage-screening task. Fitness-forduty feasibility should be tested in professional screeners and operational environments ${ }^{24}$.

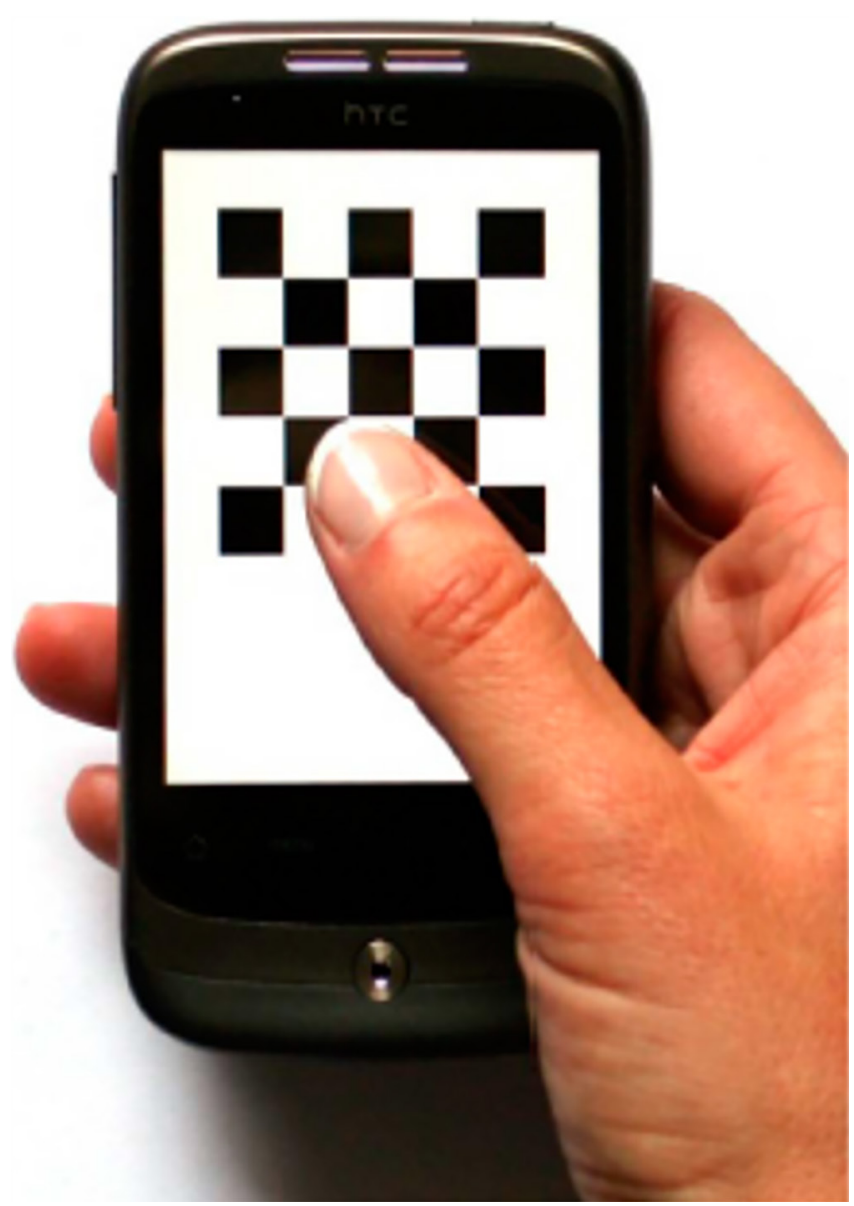

Figure 2. Pvt Touch (Kay et al., 2013) The stimulus and response occur on the same screen. In each trial, the screen starts out blank (white). After a random delay, a high contrast checkerboard pattern appears, at which point the participant provides a response touching the screen). 
PVT Touch (Kay et al., 2013): One of the newest and portable adaptations of the PVT is based on touch screen devices because it is currently more familiar and convenient. The stimulus and response occur on the same screen. The test consists of a 5-minute PVT version with random foreperiods from 1 to 10s. In each trial, the screen starts out blank (white). After a random delay, a high contrast checkerboard pattern appears, at which point the participant provides a response touching the screen. It has been compared to traditional PVT, and although the sensitivity is not as high as in the 10-minute tests, it was determined that it is valid for measuring alertness and detecting a deterioration of the performance induced by TSD, with an increase in the number of lapses, average RTs and false starts ${ }^{38}$.

PVT for touch screen devices (Arsintescu et al, NASA Ames Research, 2017): Under carefully controlled laboratory conditions, touch screen versions of the PVT yield changes in RT consistent with those recorded by computer versions of the test ${ }^{44}$. A PVT has developed in a touch screen device (fifth-generation iPod) and thirteen participants completed a 5-min PVT in three positions (on a table with index finger, handheld portrait with index finger, handheld landscape with thumb). First session has recorded with a high speed video camera ${ }^{44}$. RTs differed depending on the orientation of the device and the finger used to respond to the stimulus and it was found a substantial response latency between the actual time of an individual's touch response and the time recorded by the touch screen device ${ }^{44}$. About the PVT duration, studies show that, in those PVT of 10 minutes, the performance decreases significantly in the first 2 minutes and in the first 5 minutes. This decrease in performance is observed in the means of the RTs, in the optimal responses and the responses in the span of time. This shows that tests under 10 minutes are sensitive for the detection of sleep loss ${ }^{18}$.

It is noteworthy that although some sleep tests (such as Maintenance of Wakefulness Test and Multiple Sleep Latency
Test, not described in this review ${ }^{10,23}$ ) report sensitivity and specificity values, no such values were found on the PVT validation studies reviewed. In addition, we did not find reports of positive predictive values (to what extent a classifier or diagnostic test is able to detect) or negative predictive values (how many positive results are incorrect among all the negative cases available).

\section{CONCLUSION}

The measurement of reaction time is very useful to determine the sleep deprivation and prevent declines in the performance ${ }^{28}$.

The PVT is the gold standard test for the detection of TSD and PSL in its different versions with respect to the duration and characteristics of devices ${ }^{7}$. It has evolved from being a 10-minute test developed in large equipment to a 3-minute test that can be performed with a mobile device. These new features make it more feasible to be used in operating environments ${ }^{24}$.

Nowadays there are numerous technologies that are used for sleep deprivation assessment, but many lack scientific support that support their use. PVT validation works report to have optimal sensitivity (for the purpose of sleep deprivation), operational validity, predictive validity (ability to predict the performance capacity that is operationally relevant at a future time); reliability; specificity and generalization to be used in clinical and operational settings where tests must be brief, with minimal interference, portable and not intrusive, among other features ${ }^{6,12}$. Although the validity procedures asseverate that the tests meet all these criteria, the exact values are not informed. This detailed data is essential to determine if the test is optimal for it implementation on an specific operational environment ${ }^{12}$.

Future developments of PVT task in more portable and practical technologies should report detailed information about specificity, sensitivity, negative and positive predictive value for the detection of sleep deprivation and fatigue in operational environments to avoid accidents ${ }^{12}$.

Table 2. Comparison of the characteristics of the different PVT

\begin{tabular}{|c|c|c|c|c|c|c|c|}
\hline Task/ Test & Author & Year & Device & Duration & Background & Validity & Objective \\
\hline PVT-192 & Ambulatory Monitoring Inc. & 1984 & $\begin{array}{l}\text { Hand held self- } \\
\text { contained system }\end{array}$ & $10 \mathrm{~m}$ & $\begin{array}{l}\text { Experimental, clinical } \\
\text { \& operational }\end{array}$ & Yes & Sleep deprivation and fatigue \\
\hline Palm PVT & $\begin{array}{l}\text { Walter Reed Army Institute } \\
\text { of Research }\end{array}$ & 2005 & PDAs, Palm-Os & $5 \mathrm{~m}$ & $\begin{array}{l}\text { Experimental \& } \\
\text { Operational }\end{array}$ & Yes & Sleep deprivation \& Fatigue \\
\hline PVT-B & Basner et al. & 2011 & Computer & $3 \mathrm{~m}$ & Experimental & Yes & Sleep deprivation \\
\hline Fitness for duty & Basner \& Rubinstein & 2011 & Computer & $3 \mathrm{~m}$ & $\begin{array}{l}\text { Experimental \& } \\
\text { Operational }\end{array}$ & Yes & $\begin{array}{c}\text { Predict performance on a simulated } \\
\text { luggage-screening task }\end{array}$ \\
\hline PVT-A & Basner \& Dinges & 2012 & Computer & $6.5 \mathrm{~m}$ & Experimental & Yes & \\
\hline PC-PVT & Khitrov & 2013 & $\begin{array}{l}\text { Computer, Windows } \\
7\end{array}$ & $\begin{array}{c}5 \mathrm{~m} \text { to } \\
10 \mathrm{~m}\end{array}$ & Experimental & Yes & $\begin{array}{c}\text { Sleep deprivation \& } \\
\text { Neurobehavioral performance }\end{array}$ \\
\hline PVT Touch & Kay et al. & 2013 & Touch screen devices & $5 \mathrm{~m}$ & Experimental & Yes & $\begin{array}{l}\text { Alertness, deterioration of } \\
\text { performance induced by TSD }\end{array}$ \\
\hline $\begin{array}{l}\text { PVT for touch } \\
\text { screen devices }\end{array}$ & NASA Ames Research & 2017 & iPod & $5 \mathrm{~m}$ & Experimental & No & Fatigue \\
\hline
\end{tabular}

10 minutes are sensitive for the detection of sleep $\operatorname{loss}^{18}$. 


\section{ACKNOWLEDGMENTS}

This study was supported by a grant from the Agencia Nacional de Promoción Científica y Tecnológica (PICT Start-Up 2013-0710) and the 4th Research Project Accreditation Program (PROAPI), from the Catholic University of Argentina (UCA).

\section{REFERENCES}

1. Chattu VK, Manzar MD, Kumary S, Burman D, Spence DW, PandiPerumal SR. The Global Problem of Insufficient Sleep and Its Serious Public Health Implications. Healthcare. 2018;7(1).

2. Redeker NS, Caruso CC, Hashmi SD, Mullington JM, Grandner M, Morgenthaler TI. Workplace Interventions to Promote Sleep Health and an Alert, Healthy Workforce. Journal of clinical sleep medicine : JCSM : official publication of the American Academy of Sleep Medicine. 2019;15(4):649-57.

3. Phillips RO. A review of definitions of fatigue - And a step towards a whole definition. Transportation Research Part F: Traffic Psychology and Behaviour. 2015;29:48-56

4. Gander PH, Wu LJ, van den Berg M, Lamp A, Hoeg L, Belenky G. Fatigue Risk Management Systems. In: Kryger M, Roth T, Dement WC, editors. Principles and Practice of Sleep Medicine (Sixth Edition): Elsevier; 2017. p. 697-707.e4.

5. Uehli K, Mehta AJ, Miedinger D, Hug K, Schindler C, Holsboer-Trachsler E, et al. Sleep problems and work injuries: a systematic review and metaanalysis. Sleep Med Rev. 2014;18(1):61-73.

6. Balkin T, Bliese P, Belenky G, Sing H, Thorne D, Thomas M, et al. Comparative utility of instruments for monitoring sleepiness-related performance decrements in the operational environment. Journal of sleep research. 2004;13:219-27.

7. Lim J, Dinges D. Sleep Deprivation and Vigilant Attention. Annals of the New York Academy of Sciences. 2008;1129:305-22.

8. Killgore WDS. Effects of sleep deprivation on cognition. Progress in brain research. 2010;185:105-29.

9. Basner M, Dinges D. Maximizing Sensitivity of the Psychomotor Vigilance Test (PVT) to Sleep Loss. Sleep. 2011;34:581-91.

10. Coelho F, Narayansingh M, Murray B. Testing sleepiness and vigilance in the sleep laboratory. Current opinion in pulmonary medicine. 2011;17:406-11.

11. Eckner J, Richardson J, Kim H, Lipps D, Ashton-Miller J. A Novel Clinical Test of Recognition Reaction Time in Healthy Adults. Psychological assessment. 2012;24:249-54.

12. Dawson D, Searle A, Paterson J. Look before you (s)leep: Evaluating the use of fatigue detection technologies within a fatigue risk management system for the road transport industry. Sleep medicine reviews. 2013;18.

13. Gabehart RJ, Van Dongen HPA. Circadian Rhythms in Sleepiness, Alertness, and Performance. In: Kryger M, Roth T, Dement WC, editors. Principles and Practice of Sleep Medicine (Sixth Edition)2017. p. 388-95.e5.

14. Banks S, Landon LB, Dorrian J, Waggoner LB, Centofanti SA, Roma PG, et al. Effects of fatigue on teams and their role in 24/7 operations. Sleep Med Sleep Medicine Reviews. 2019;48:101216.

15. Durmer J, Dinges D. Neurocognitive Consequences of Sleep Deprivation. Seminars in neurology. 2005;25:117-29.

16. Jones M, Dunican IC, Murray K, Peeling P, Dawson B, Halson S, et al. The psychomotor vigilance test: a comparison of different test durations in elite athletes. Journal of Sports Sciences. 2018;36:1-5.

17. Van Dongen H, Maislin G, Mullington J, Dinges D. The Cumulative Cost of Additional Wakefulness: Dose-Response Effects on Neurobehavioral Functions and Sleep Physiology From Chronic Sleep Restriction and Total Sleep Deprivation. Sleep. 2003;26:117-26.

18. Loh S, Lamond N, Dorrian J, Roach G, Dawson D. The validity of psychomotor vigilance tasks of less than 10-minute duration. Behavior research methods, instruments, \& computers: a journal of the Psychonomic Society, Inc. 2004:36:339-46.

19. Hansen D, Layton M, Riedy S, Dongen H. Psychomotor Vigilance Impairment During Total Sleep Deprivation Is Exacerbated in SleepOnset Insomnia. Nature and Science of Sleep. 2019;Volume 11:401-10.

20. Dawson D, Reid K. Fatigue, alcohol and performance impairment. Nature. 1997;388:235.

21. Basner M, Dinges D. An Adaptive-Duration Version of the PVT Accurately Tracks Changes in Psychomotor Vigilance Induced by Sleep Restriction. Sleep. 2012;35:193-202.

22. Patanaik A, Kwoh C-K, Chua E, Gooley J, Chee M. Classifying Vulnerability to Sleep Deprivation Using Baseline Measures of Psychomotor Vigilance. Sleep. 2014;38.
23. Shahid A, Shen J, Shapiro CM. Measurements of sleepiness and fatigue Journal of psychosomatic research. 2010;69(1):81-9.

24. Basner M, Rubinstein J. Fitness for duty: a 3-minute version of the Psychomotor Vigilance Test predicts fatigue-related declines in luggagescreening performance. Journal of occupational and environmental medicine. 2011;53(10):1146-54.

25. Glenville M, Broughton R, Wing A, Wilkinson R. Effects of sleep deprivation on short duration performance compared to the Wilkinson Auditory Vigilance Task. Sleep. 1978;1:169-76.

26. Dinges DF, Powell JW. Microcomputer analyses of performance on a portable, simple visual RT task during sustained operations. Behavior Research Methods, Instruments \& Computers. 1985;17(6):652-5.

27. Crocetta TB, Viana RL, Silva DE, Monteiro CBdM, Arab C, Andrade A. Validity of Software for Measurement of Total Reaction Time with Simple Stimulus -Trt_S 2012. Journal of Human Growth and Development. 2014;24(3):295

28. Jaffe D, Hewit J, Comstock K, Bedard A. Effects of Sleep on Balance Control and Reaction Time to Visual Stimuli. COJ Technical \& Scientific Research. 2018;1(1):1-5.

29. Woods D, Wyma J, Yund E, Herron T, Reed B. Factors influencing the latency of simple reaction time. Frontiers in human neuroscience. 2015;9:131.

30. Pramanik T, Dhakal R, Pandit R. Visual Reaction Time in People with and without Diabetes - A Comparative Study. Nepal Medical College Journal. 2019;21:100-3.

31. Eckner JT, Whitacre RD, Kirsch NL, Richardson JK. Evaluating a clinical measure of reaction time: an observational study. Perceptual and motor skills. 2009;108(3):717-20.

32. Jung CM, Ronda JM, Czeisler CA, Wright KP, Jr. Comparison of sustained attention assessed by auditory and visual psychomotor vigilance tasks prior to and during sleep deprivation. J Sleep Res. 2011;20(2):348-55.

33. Petrilli R, Jay S, Dawson D, Lamond N. The Impact of Sustained Wakefulness and Time-of-day on OSPAT Performance. Industrial health 2005;43:186-92.

34. Matsangas P, Shattuck N. Agreement between the 3-minute Psychomotor Vigilance Task (PVT) Embedded in a Wrist-worn Device and the Laptopbased PVT. Human Factors and Ergonomics Society Annual Meeting Proceedings. 2018;62:666-70.

35. Hoermann H. The Psychomotor Vigilance Test: Sources of State and Trait Variance. 17th International Symposium on Aviation Psychology; Ohio, USA2013. p. 657-62.

36. Zhang C, Varvarigou V, Parks P, Gautam S, Bueno A, Malhotra A, et al. Psychomotor Vigilance Testing of Professional Drivers in the Occupational Health Clinic A Potential Objective Screen for Daytime Sleepiness. Journal of occupational and environmental medicine. 2011;54:296-302.

37. Dorrian J, Lamond N, Dawson D. The ability to self-monitor performance when fatigued. Journal of sleep research. 2000;9:137-44.

38. Kay M, Rector K, Consolvo S, Greenstein B, Wobbrock J, Watson NF, et al. PVT-Touch: Adapting a Reaction Time Test for Touchscreen Devices. 7th International Conference on Pervasive Computing Technologies for Healthcare and Workshops; 2017; Venice, Italy: Pervasive Health; 2013. p. 248-51.

39. Matthews R, Ferguson S, Sargent C, Zhou X, Kosmadopoulos A, Roach G. Using interstimulus interval to maximise sensitivity of the Psychomotor Vigilance Test to fatigue. Accident Analysis \& Prevention. 2017;99:406-10.

40. Grant D, Honn K, Layton M, Riedy S, Dongen H. 3-minute smartphonebased and tablet-based psychomotor vigilance tests for the assessment of reduced alertness due to sleep deprivation. Behavior Research Methods. 2016;49.

41. Thorne D, Johnson D, Redmond D, Sing H, Belenky G, Shapiro J. The Walter Reed palm-held psychomotor vigilance test. Behavior research methods. 2005;37:111-8.

42. Khitrov M, Laxminarayan S, Thorsley D, Ramakrishnan S, Rajaraman S, Wesensten N, et al. PC-PVT: A platform for psychomotor vigilance task testing, analysis, and prediction. Behavior research methods. 2013;46.

43. Drake C, Roehrs T, Burduvali E, Bonahoom A, Rosekind M, Roth T Effects of rapid versus slow accumulation of eight hours of sleep loss Psychophysiology. 2001;38:979-87.

44. Arsintescu L, Mulligan J, Flynn-Evans E. Evaluation of a Psychomotor Vigilance Task for Touch Screen Devices. Human factors. 2017;59:18720816688394.

45. Basner M, Mollicone D, Dinges DF. Validity and sensitivity of a brief psychomotor vigilance test (PVT-B) to total and partial sleep deprivation. Acta Astronautica. 2011;69(11-12):949-59. 


\section{The effect of pubertal development on the sleep-wake cycle}

Jorge Borrani *

Gonzalo Guerra

Aída García

Candelaria Ramírez

Pablo Valdez

Universidad Autónoma de Nuevo León, Laboratory of Psychophysiology. Monterrey, Nuevo León, México.

\begin{abstract}
Objective: To analyze if the delay of the sleep-wake cycle is the effect of pubertal development, independently of age or school grade. Methods: Girls and boys between 4th and 6th grade were divided into a low pubertal stage and a high pubertal stage group. Age and school grade were controlled to isolate the effect of puberty by pairing the groups on these variables. The regular bedtime, waking time, sleep duration and daytime sleepiness were obtained through questionnaires. A Pubertal Developmental Scale with questions about bodily changes was used to determine pubertal stage. Results: Girls in high pubertal stages reported weekend bedtimes an hour later than girls in low puberty stages (low pubertal stage: $22: 36 \pm 2: 11 \mathrm{~h}$, high pubertal stage: $23: 37 \pm 1: 27$ $\mathrm{h}, \mathrm{U}=215, \mathrm{p}<0.05)$; there were no differences on waking time or sleep duration. Boys of different pubertal development did not have differences on any sleep parameter. Girls had a longer sleep duration compared to boys on weekends, but not on weekdays. Discussion: The bedtime delay in girls is the effect of pubertal development, not of age or school grade. There was no effect on boys due to their lower pubertal development at this age. Variability in pubertal development creates disparities in the sleep patterns of pubescent girls, even of the same age and school grade. Acknowledging this delay can help to create school and sports schedules in which young people are fully awake and alert. In research, it is crucial to consider pubertal development while studying sleep in children and adolescents.
\end{abstract}

Keywords: Adolescent; Puberty; Sleep

\author{
Corresponding author: \\ Jorge Borrani \\ E-mail: jorgeborrani@gmail.com \\ jorgeborrani@yahoo.com.mx
}




\section{INTRODUCTION}

The phase and period of the sleep-wake cycle change considerably during the human lifespan, one notable change is that young people tend to stay up later during the night and wake up later in the day ${ }^{1,2}$.

Even though this change in the sleep-wake cycle is usually related to adolescence, the physiological phenomenon driving the delay of the sleep phase is puberty. Other mammalian species also experience these changes in sleep habits ${ }^{3}$, which implies that they are not just a consequence of an increase in the use of technology, or a reduction in parental control ${ }^{4}$.

Puberty is a period of massive physiological and psychological changes, caused by the activation of the hypothalamic-pituitary-adrenal axis and the hypothalamicpituitary-gonadal axis, which increases the production of androgens, estrogens and progesterones, among other hormones. These hormones are usually related to sexual maturation and their most evident effects are the appearance of secondary sex characteristics, but they also have important effects on the nervous system and sleep ${ }^{5,6}$.

Sleep is produced and maintained by brain structures that are thought to be susceptible to pubertal hormones, for example, a characteristic that defines the sleep state in the brain is the appearance of EEG slow-wave activity, which starts declining when pubertal hormones initiate a synaptic pruning of the brain? Similarly, there is also a reduction of nighttime melatonin release that negatively relates with the pubertal increase in luteinizing hormone ${ }^{8}$. The increase in hormone production during puberty is thought to delay the sleep-wake cycle in adolescents through one, or a combination of the following mechanisms: by changing the period of the circadian clock, by changing the sensitivity of the system to light, or by reducing homeostatic sleep pressure?.

Although the delay of the sleep-wake cycle in adolescents is attributed to the effect of pubertal hormones ${ }^{10}$, there is scarce behavioral evidence showing that adolescents, in fact, delay their sleep as their puberty advances. This lack of evidence is because most studies document this delay by comparing age groups of younger and older adolescents, which proves the effect of age but not the effect of puberty? To analyze the effect of puberty it is necessary to determine the advance of this developmental process, one way to do this is to measure the amount of pubertal hormones in the body, and the other is to measure the effects of such hormones.

The Tanner Scale is the most common evaluation system to determine the advance of pubertal development, without using blood or saliva samples. This scale is completed by a professional via visual inspection, it classifies growth of axillary and pubic hair and the development of breast and testes in five stages, from prepubertal to post-puberal ${ }^{11}$. Medical inspection of secondary sexual characteristics is difficult to implement in school settings, therefore self-report scales have been developed to assess a great number of participants in a non-invasive fashion ${ }^{12}$. Self-report scales of pubertal development also classify body, skin and voice changes in similar stages, ranging from a moment of no pubertal change to when changes are complete ${ }^{13}$.
Although puberty and age correlate, that is, older children tend to be more advanced in their pubertal development than younger ones, puberty onset in humans has a normal window of appearance of around six years, even in children under similar environmental factors ${ }^{14}$. For example, in Mexico the mean age for menarche is 12.5 years of age, but it may appear from 9.5 until 15.5 years of age, and still be considered a normal pubertal onset $^{15}$. Boys have a similar variability in pubertal onset, but it is delayed approximately one year in comparison to girls. In consequence, if the delay and extension of the sleep-wake cycle is due to puberty, there is no fixed age for these changes to begin.

This means that even among children of the same age and school grade, children with a more advanced puberty could have a more delayed sleep phase, making it harder for them to comply with early morning activities. It is well documented that school schedules interfere with adolescent sleep schedules ${ }^{16}$, and that there is a marked delay from weekdays to weekends in high school and college students, which is thought to be a recovery of the weekday sleep restriction ${ }^{17}$. Again, this restriction and recovery has been studied through age groups and not through pubertal development.

To analyze these changes on the sleep-wake cycle of adolescents, it is crucial to dissociate puberty from other factors that may influence sleep habits. For example, age drives many other developmental processes that happen independently of puberty, therefore it is always important to control this factor in adolescents. Also, as children advance in school grade there is a documented reduction of sleep time, probably due to an increase in academic workload ${ }^{18}$. The advance from one grade to the next may also change school schedules and social activity, which are important influences on the sleep-wake cycle of adolescents. Therefore, it is important to control age and school grade when analyzing changes in the adolescent sleep-wake cycle.

Only a few of the studies that analyze the adolescent sleep-wake cycle take into account pubertal development and of those that do, not all control age or school grade. In a longitudinal study by Andrade, Benedito-Silva, Domenice, Arnhold and Menna-Barreto ${ }^{1}$ participants advanced Tanner stages every semester that was surveyed, similar to their delay in sleep onset. Although this suggests a relationship between pubertal development and sleep habits, there were no analysis to link these variables.

In a classic study by Carskadon, Vieira and Acebo ${ }^{4}$ five hundred and fifty 6 th graders were recruited. This sample criteria partially controlled age, since it left a relatively small range of 11 to 12 years of age, and it effectively controlled school grade. Pubertal development was classified in two pubertal stages for boys ("no changes yet" and "incomplete changes"), and three stages for girls ("no changes", "incomplete changes" and "marked complete changes"); bedtimes were divided into early and late bedtimes. A relationship was found between later bedtimes and a higher stage of puberty. Nevertheless, it is not clear if this relation is independent of age. During puberty, a difference of one year can cause great changes, so it could have happened that participants with earlier bedtimes were all 11 years old and those with later bedtimes were all 12 years old. 
It is not clear how much later were the bedtimes of participants in higher puberty stages, from the bedtimes of participants with lower pubertal development. It was also not clear how much more advanced in pubertal development were the participants with late bedtimes, compared to the participants with early bedtimes.

Another longitudinal study showed, through actigraphy, that participants with more pubertal advance between two moments of the study, were the ones that delayed their sleep onset the most. This study also found a decrease in sleep duration, which could happen if bedtime is delayed and waking time is not delayed as well. Age in this study was also in a tight range from 9.9 to 11.2 years of age, and it was further controlled statistically by partialling age out of the correlation model, nevertheless, school grade was not controlled ${ }^{19}$.

Even though there is evidence to support the hypothesis that the delay of the sleep-wake cycle in adolescents is the effect of pubertal development, there are no studies that isolate the effect of puberty by directly controlling age or school grade. Therefore, the objective of this study is to analyze if the delay of the sleep-wake cycle is the effect of pubertal development, independently of age or school grade.

\section{MATERIAL AND METHODS}

\section{Participants}

For this study, eighty-two children between 9 and 12 years of age were recruited from fourth, fifth and sixth grade of elementary school (age: $10.32 \pm 0.9$ years; school grade: $5.0 \pm 0.8$ years; mean \pm standard deviation). This sample was divided into a group of 52 girls (age: $10.52 \pm 0.8$ years; school grade: $4.7 \pm 0.7$ years) and a group of 30 boys (age: $11.26 \pm 0.9$ years; education: $5.4 \pm 0.7$ school years). Participants attended school from 8:30 h to $12: 30 \mathrm{~h}$.

Girls were divided into two groups of different pubertal development (low pubertal stage $1.5 \pm 0.46$; high pubertal stage: $3.6 \pm 0.57 ; \mathrm{U}=7.0, \mathrm{p}<0.001$ ), as well as boys (low pubertal stage: $1.6 \pm 0.24$; high pubertal stage: $2.2 \pm 0.45 ; \mathrm{U}=23, \mathrm{p}<0.001)$. In order to isolate the effect of puberty from age and school grade, the low and high pubertal stage groups were paired by age (Girls: low pubertal stage $10.39 \pm 0.79$ years old, high pubertal stage $10.65 \pm 0.81$ years old; $U=316.50$, NS; Boys: low pubertal stage $11.25 \pm 1.05$ years old, high pubertal stage $11.27 \pm 0.83$ years old; $\mathrm{U}=112.50, \mathrm{NS})$ and school grade (Girls: low pubertal stage 4.65 \pm 0.74 school years, high pubertal stage $4.92 \pm 0.79$ school years; $\mathrm{U}=274.50$, NS; Boys: low pubertal stage $5.4 \pm 0.73$ school years, high pubertal stage $5.4 \pm 0.73$ school years; $U=112.50$, NS).

Participants were paired case by case, for example, a 10 -year-old girl in the 5 th grade, with a puberty stage score of 2 , was paired with another 10-year-old girl that is also in the 5 th grade, but that had the highest possible puberty stage, in this case a score of 4 . The average difference in puberty stage between pairs of girls was $2.0 \pm 0.69$, but for pairs of boys the average difference was $0.6 \pm 0.55$. This difference is very small, so pubertal stage differences were not expected in boys.
Participants slept 8:56 \pm 1:33 h on weekdays and 10:19 $\pm 2: 25 \mathrm{~h}$ on weekends, they had no sleep disorders or other sleep complaints at the moment of the application. All were without risk of having suffered brain damage, and were not using medications that could affect sleep or the nervous system in general.

\section{Instruments and procedures}

A general data questionnaire was used to collect information about health, brain damage risk, gender and age. The Sleep Timing Questionnaire ${ }^{20}$ was used to collect the usual bedtime and waking time of the children, during weekdays and weekends. The Epworth Sleepiness Scale ${ }^{21}$ is a questionnaire about the probability of falling asleep in different situations during the day, it was used to measure sleepiness that may occur because of sleep deprivation.

The Pubertal Development Scale (PDS) ${ }^{12}$ is a Likerttype questionnaire that is answered by the participant, it assesses the physical development of participants in the areas of height, changes in skin (appearance of acne vulgaris), pubic hair growth and axillary hair growth. Male participants were also asked about changes in their voice and facial hair growth; female participants were also asked about breast growth and the appearance of their menstrual period. Through these questions each characteristic is scored as follows, 1: no change yet, 2: change has barely begun, 3: change is definitely underway, and 4: change is completed; menarche is scored 1: menarche has not appeared or 4: menarche has already appeared. These scores were then averaged to obtain a final score of pubertal development.

All tasks and questionnaires were administered as an interview and were applied individually at the school the participants attended, during their regular schedule.

\section{Data Analysis}

A Mann-Whitney U test was used to compare daily sleepiness and sleep parameters such as bedtime, waking time and sleep duration, between low and high pubertal stage groups and also between girls and boys. A Wilcoxon $\mathrm{T}$ test was used to compare the same sleep parameters between weekdays and weekends.

\section{RESULTS}

The girls in the high pubertal stage group went to sleep an hour later on weekends than the girls in the low pubertal stage group (low pubertal stage: $22: 36 \pm 2: 11 \mathrm{~h}$, high pubertal stage: $23: 37 \pm 1: 27 \mathrm{~h}, \mathrm{U}=215, \mathrm{p}<0.05)$. Waking time and sleep duration did not show significant differences during weekends. During weekdays, girls showed no pubertal stage differences on sleep parameters (Figure 1). Among boys there were no differences between low and high pubertal stage groups on any sleep parameter, either on weekdays or on weekends (Figure 2). 
Girls

Low pubertal stage

High pubertal stage
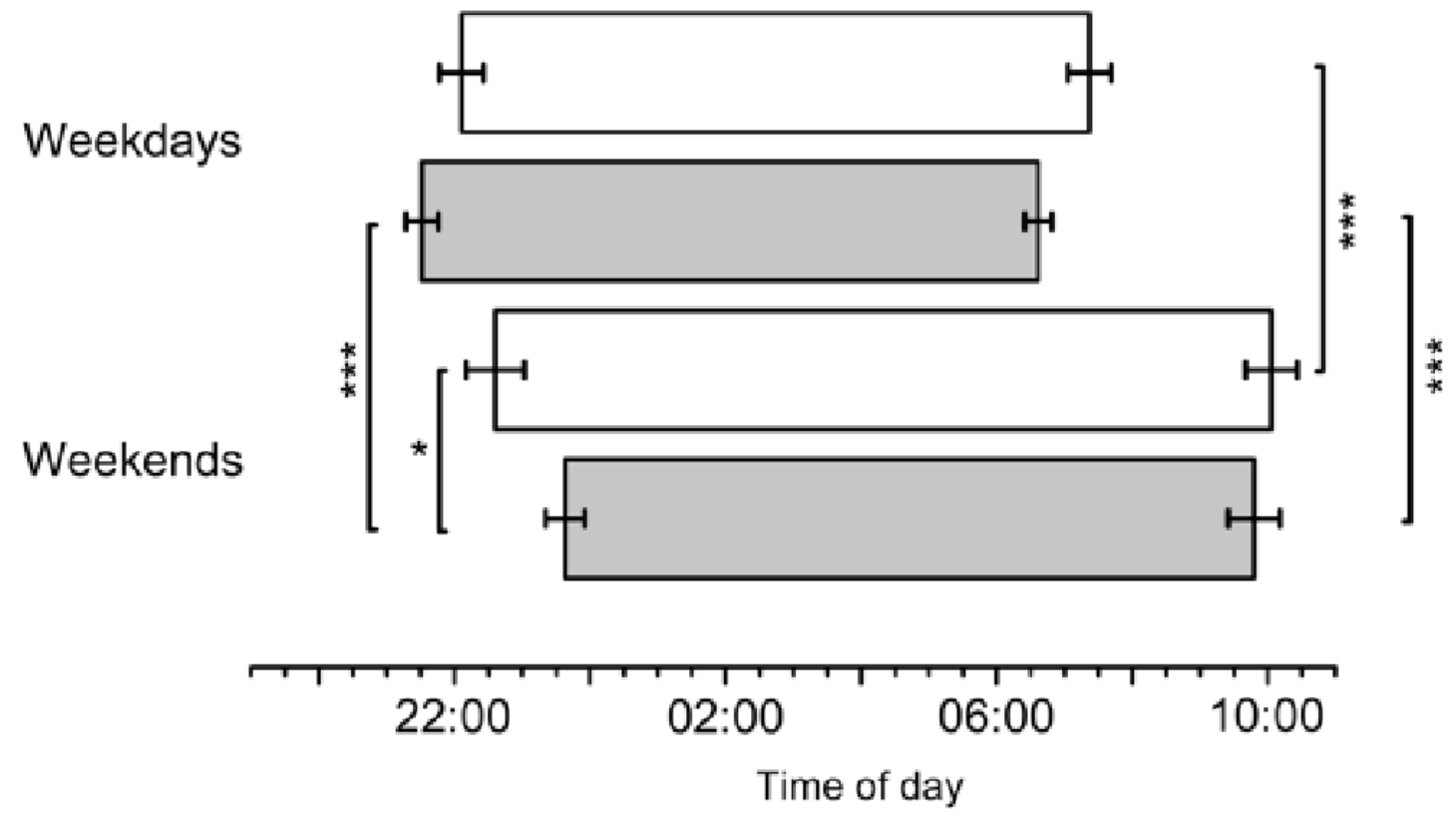

Figure 1. Comparison of the sleep-wake cycle parameters in girls between the low pubertal stage group and the high pubertal stage group, during weekdays and weekends. ${ }^{*} \mathrm{p}<0.05{ }^{* * *} \mathrm{p}<0.001$

\section{Boys}
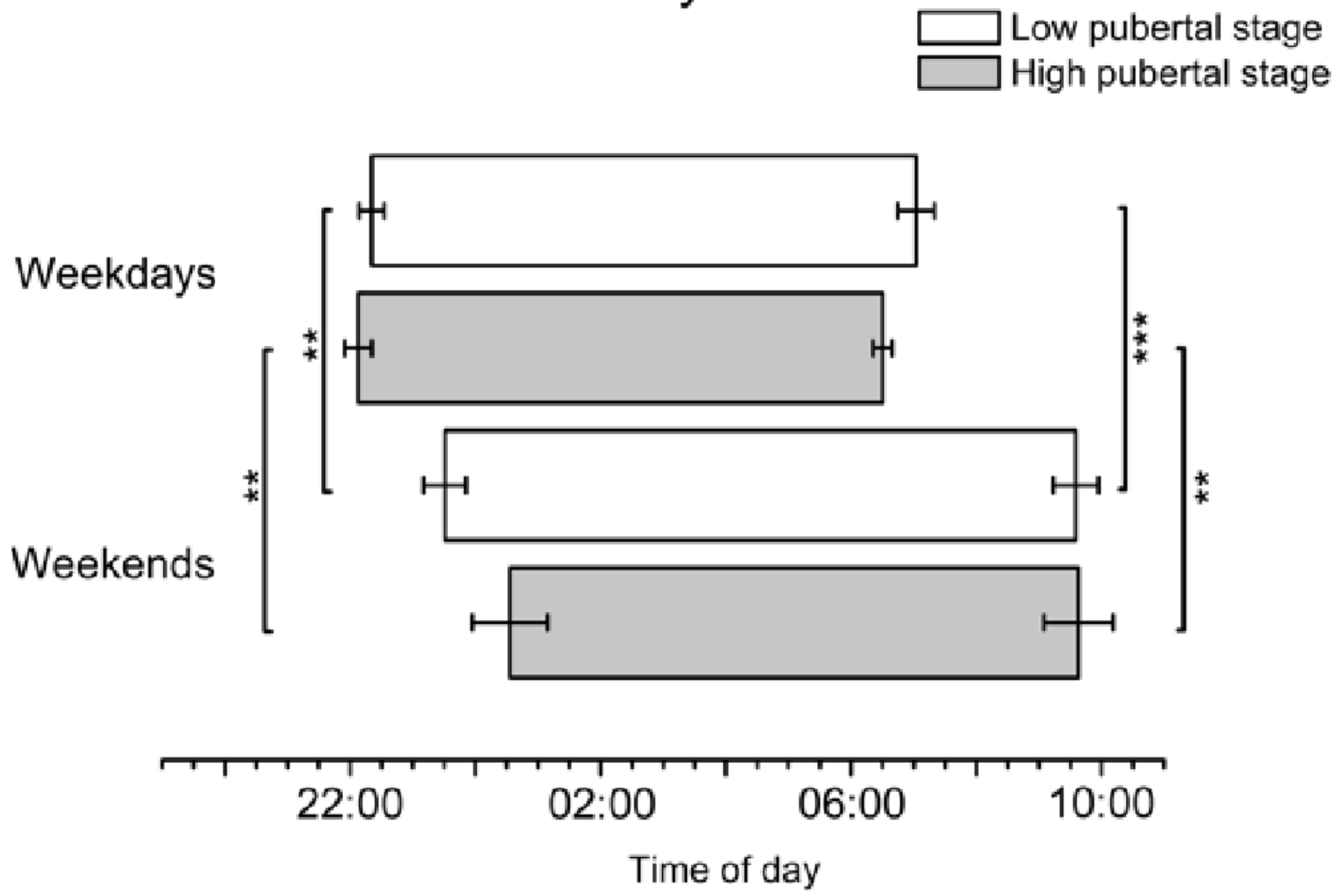

Figure 2. Comparison of the sleep-wake cycle parameters in boys between the low pubertal stage group and the high pubertal stage group, during weekdays and weekends. ${ }^{* *} \mathrm{p}<0.01{ }^{* * *} \mathrm{p}<0.001$ 
Comparing weekdays (WD) to weekends (WE), girls in the low pubertal stage group delayed their waking time (WD: 7:21 \pm 1:64 h, WE: 10:03 \pm 1:56 h, $T=6.0, \mathrm{p}<0.001)$ and had a longer sleep duration on the weekends (WD: 9:15 \pm 2:00 $\mathrm{h}$, WE: 11:27 \pm 2:16 h, $\mathrm{T}=13.5, \mathrm{p}<0.001$ ), without differences in bedtime. Girls in the high pubertal stage group delayed their bedtime (WD: 21:30 \pm 1:12 h, WE: 23:37 \pm 1:27 h, T = $0.0, \mathrm{p}<0.001$ ) and their waking time (WD: 6:36 \pm 1:01 h, WE: $9: 47 \pm 1: 56 \mathrm{~h}, \mathrm{~T}=0.0, \mathrm{p}<0.001)$ during weekends, and had no differences in sleep duration (Figure 1).

In boys, these same comparisons showed that low pubertal stage boys delayed their bedtime (WD: 22:20 \pm 0:46 h, WE: 23:30 \pm 1:18 $\mathrm{h}, \mathrm{T}=0.0, \mathrm{p}<0.01$ ), their waking time (WD: 7:01 \pm 1:07 h, WE: 9:35 $\pm 1: 23 \mathrm{~h}, \mathrm{~T}=1.0, \mathrm{p}<0.001$ ), and had longer sleep durations on the weekend (WD: 8:41 \pm 0:57 h, WE: 10:02 \pm 1:40 h, $\mathrm{T}=9.0, \mathrm{p}<0.05)$. High pubertal stage boys also delayed their bedtime (WD: 22:07 \pm 0:50 h, WE: 00:33 $\pm 2: 19 \mathrm{~h}, \mathrm{~T}=0.0$, $\mathrm{p}<0.001$ ) and waking time (WD: 6:30 \pm 0:34 h, WE: 9:37 \pm 2:08 h, $\mathrm{T}=0.0, \mathrm{p}<0.001$ ), but had no change in sleep duration (Figure 2).

When comparing participants by gender, regardless of pubertal stage, there were no differences in bedtime or waking time between boys and girls. Nevertheless, girls had a longer sleep duration on weekends in comparison to boys (girls: 10:45 \pm 2:18 h, boys: 9:31 $\pm 2: 25 \mathrm{~h}, \mathrm{U}=541, \mathrm{p}<0.05)$; this difference disappears when comparing sleep parameters on weekdays. Also, there were no differences when gender comparisons were made between girls and boys of low pubertal stage, and the same happened when comparing girls and boys of high pubertal stage.

The average score for the whole sample in daily sleepiness was $9.03 \pm 3.93$, which is three points above the normal limit of $\mathrm{six}^{21}$. Nevertheless, there were no differences when comparing sleepiness by pubertal development or by gender.

\section{DISCUSSION}

The results show that, at least in girls, the delay in bedtime that is characteristic of adolescence is the effect of pubertal development, independently of age and school year. This bedtime delay means that girls in the same age and in the same school grade can be two pubertal stages apart, and thus have a difference of about one hour in their bedtime, which is a considerable delay $^{22}$. Girls, regardless of pubertal stage, also sleep longer on the weekend compared to boys, this could be interpreted as a greater need for sleep in the gender that is typically more advanced in pubertal development, but it could also reflect other gender differences that increase their sleep on weekends.

The effect of pubertal development was not strong enough to be evident on waking time. Other studies report changes in waking time when analyzing high school or college students $^{23}$, but in this study participants are barely entering puberty, therefore the effect on waking time is probably not yet manifested. The lack of differences on sleep duration is expected, since other studies ${ }^{24,17}$ have already established that, although there is a delay in the sleep-wake cycle of adolescents, there is no overall change in sleep duration when weekdays and weekends are considered.
These results support what other reports had suggested, that the delay of the sleep phase is due to puberty. Nevertheless, the improved control of age and school year in this study further confirms that this delay in the sleep-wake cycle of pubescent girls is independent of other age-related changes, and also independent of changes related to school grade advance, such as an increased academic workload.

On boys, there was no significant effect of pubertal development on their sleep-wake cycle, probably due to the fact that boys start puberty later, and therefore show a smaller range of pubertal stages during these ages, which is a common issue in these studies ${ }^{25,26}$. The effect of this small range of pubertal stages was that comparisons were made between participants in very similar stages of puberty. To obtain a wider range of pubertal stages in boys it is necessary to recruit participants in middle school. Also, boys have been found to be less accurate when reporting their pubertal status ${ }^{12}$, which could also confound the pubertal stage comparisons made here. The present results should be replicated taking into account other variables, such as body mass index, sibling order, the presence of siblings in the same room, and the use of light-emitting screens ${ }^{27}$.

In sum, these results indicate that the bedtime delay of pubescent girls is the effect of puberty, and not of age or school grade. Therefore, girls that are more advanced in their pubertal development delay their bedtime sleep schedule, compared to other girls of the same age and in the same school year. This effect was observed only during weekends, days when they can choose their bedtime without the constraint of school schedules.

In this study, all participants had a delay in bedtime from weekdays to weekends, similar to that reported in the literature ${ }^{1,28}$. Low puberty girls and boys showed an increase in sleep duration from weekdays to weekends, while the other groups did not show a sleep extension. This suggests that there is no pubertal effect on the weekend extension of sleep. The index for sleep deprivation of this study was daytime sleepiness, but no differences were found with pubertal development, probably related to the absence of differences in sleep duration.

It is crucial that everyone in touch with children and adolescents (educators, coaches, parents and public policy makers) acknowledges that there are individual differences in pubertal development, and thus, different sleep patterns even within the same age group and school grade. Through this understanding, better decisions can be taken about school and sport training schedules, in order to ensure young people are awake and alert during their activities. Educating children and adolescents about the changes in their sleep-wake cycle has been related to the development of healthy sleep habits, which can help them to better cope with the conflict between their sleep pattern and socially imposed schedules for school and entertainment ${ }^{29}$. Similarly, it is crucial that researchers consider puberty as a central factor in sleep-wake cycle changes, and hence take it into consideration when investigating sleep in children and adolescents. The measurement of puberty is a complex subject in itself, and innovative approaches need to be made to fully integrate it into sleep research. 


\section{CONCLUSION}

The delay of bedtime in pubescent girls is the effect of pubertal development, independently of age and school year.

\section{REFERENCES}

1. Andrade M, Benedito-Silva A, Domenice S, Arnhold I, Menna-Barreto L. Sleep characteristics of adolescents: A longitudinal study. J Psychosom Res. 1993;14(5):401-6.

2. Thorleifsdottir B, Björnsson JK, Benediktsdottir B, Gislason T, Kristbjarnarson H. Sleep and sleep habits from childhood to young adulthood over a 10-year period. J Psychosom Res. 2002;53(1):529-37.

3. Hagenauer MH, Lee TM. Adolescent sleep patterns in humans and laboratory animals. Horm Behav. 2013;64(2):270-9.

4. Carskadon MA, Vieira C, Acebo C. Association between Puberty and Delayed Phase Preference. Sleep. 1993;16(3):258-62.

5. Blakemore S-J, Burnett S, Dahl RE. The role of puberty in the developing adolescent brain. Hum Brain Mapp. 2010;31(6):926-33.

6. Goddings A-L, Mills KL, Clasen LS, Giedd JN, Viner RM, Blakemore S-J. The influence of puberty on subcortical brain development. NeuroImage. 2014;88:242-51.

7. Campbell IG, Grimm KJ, de Bie E, Feinberg I. Sex, puberty, and the timing of sleep EEG measured adolescent brain maturation. Proc Natl Acad Sci U S A. 2012;109(15):5740-3.

8. Waldhauser F, Frisch H, Waldhauser M, Weiszenbacher G, Zeitlhuber U, Wurtman, R. Fall in nocturnal serum melatonin during prepuberty and pubescence. Lancet. 1984;323(8373):362-365.

9. Colrain IM, Baker FC. Changes in Sleep as a Function of Adolescent Development. Neuropsychol Rev. 2011;21(1):5-21.

10. Hagenauer MH, Perryman JI, Lee TM, Carskadon MA. Adolescent Changes in the Homeostatic and Circadian Regulation of Sleep. Dev Neurosci. 2009;31(4):276-84.

11. Dorn LD, Biro FM. Puberty and Its Measurement: A Decade in Review. J Res Adolescence. 2011;21(1):180-9.

12. Petersen AC, Crockett L, Richards M, Boxer A. A self-report measure of pubertal status: Reliability, validity, and initial norms. J Youth Adolescence. 1988;17(2):117-33.

13. Coleman L, Coleman J. The measurement of puberty: a review. J Adolescence. 2002;25(5):535-50.

14. Parent A-S, Teilmann G, Juul A, Skakkebaek NE, Toppari J, Bourguignon J-P. The Timing of Normal Puberty and the Age Limits of Sexual
Precocity: Variations around the World, Secular Trends, and Changes after Migration. Endocr Rev. 2003;24(5):668-93.

15. Robles C, Altamirano N. Pubertad precoz y pubertad retrasada. Cuándo y cómo tratarlas. Acta Pediatr Mex. 2003;1524(2):130-44.

16. Crowley SJ, Carskadon MA. Modifications to Weekends Recovery Sleep Delay Circadian Phase in Older Adolescents. Chronobiol Inter. 2010;27(7):1469-92.

17. Valdez P, Ramírez C, García A. Delaying and Extending Sleep During Weekends: Sleep Recovery or Circadian Effect? Chronobiol Inter. 1996;13(3):191-8.

18. Shinkoda H, Matsumoto K, Park YM, Nagashima H. Sleep-wake habits of schoolchildren according to grade. Psychiat Clin Neuros. 2000;54(3):287-9.

19. Sadeh A, Dahl RE, Shahar G, Rosenblat-Stein S. Sleep and the Transition to Adolescence: A Longitudinal Study. Sleep. 2009;32(12):1602-9.

20. Monk TH, Buysse DJ, Kennedy KS, Potts JM, DeGrazia JM, Miewald JM. Measuring Sleep Habits Without Using a Diary: The Sleep Timing Questionnaire. Sleep. 2003;26(2):208-12.

21. Johns MW. A New Method for Measuring Daytime Sleepiness: The Epworth Sleepiness Scale. Sleep. 1991;14(6):540-5.

22. Sadeh A, Gruber R, Raviv A. The Effects of Sleep Restriction and Extension on School-Age Children: What a Difference an Hour Makes. Child Dev. 2003;74(2):444-55.

23. Crowley SJ, Acebo C, Carskadon MA. Sleep, circadian rhythms, and delayed phase in adolescence. Sleep Med. 2007;8(6):602-12.

24. Anders TF, Carskadon MA, Dement WC, Harvey K. Sleep habits of children and the identification of pathologically sleepy children. Child Psych Hum Dev. 1978;9(1):56-63.

25. Carskadon, MA \& Acebo, C. A Self-Administered Rating Scale for Pubertal Development. J Adolescent Health. 1993;14:190-195.

26. Knutson KL. The association between pubertal status and sleep duration and quality among a nationally representative sample of U. S. Adolescents. Am J Hum Biol. 2005;17(4):418-24.

27. Carskadon MA. Sleep in Adolescents: The Perfect Storm. Pediatr Clin N Am. 2011;58(3):637-47.

28. National Sleep Foundation. Sleep in America Poll: Summary of findings. 2006. [cited 2020 January 16] Available from: https://www.sleepfoundation. org/sites/default/files/inline-files/2006_summary_of_findings.pdf

29. Sousa IC, Souza JC, Louzada FM, Azevedo CVM. Changes in sleep habits and knowledge after an educational sleep program in 12th grade students: Sleep education program for adolescents. Sleep Biol Rhythms. 2013;11(3):144-53. 


\section{Shift work-related health problems: causes, effects, and light-based interventions}

Liliana Bustos-González *

Centro Interdisciplinario de Neurociencia de Valparaíso, Facultad de Ciencias, Universidad de Valparaíso, Gran Bretaña 1111, Valparaíso 2360102, Chile.

\begin{abstract}
Our modern society and global economy require individuals to work under non-standard schedules to provide twenty-four-hour coverage for a large number of essential services. Despite the importance of these non-standard work schedules for production and logistical demands, they have a significant negative impact on the health of workers. Shift work and night work are recognized as risk factors for the development of a myriad of health-related problems due to the misalignment of circadian rhythms, chronic sleep deprivation, and exposure to light at night. The aim of this review is to provide an overview of the factors contributing to the detrimental effects of shift work on health and to highlight targeted interventions aimed at re-synchronizing the circadian system of those who work under shift schedules.
\end{abstract}

Keywords: Circadian Rhythms; Circadian Misalignment; Sleep; Light At Night; Light Therapy 


\section{INTRODUCTION}

Modern society and the global economy demand twentyfour-hour services. Today, shift-work allows industries, businesses, and essential services to operate continuously to provide twentyfour-hour availability of almost everything. Therefore, shift work, defined as any form of work arrangement in which workers succeed each other to perform the same job at different times throughout the day (Council Directive 2003/88/EC, 2003), is an essential and necessary part of our current society. European and American work surveys suggest that between $17 \%$ and $27 \%$ of the workforce in Europe and America is engaged in shift work, respectively, which includes night and rotating shifts ${ }^{2,3}$. In Chile, a survey conducted by the government's Labor Bureau (2014) indicated that $11 \%$ of men and $5 \%$ of women in the private industry work under such schedules, with indications that these rates will increase in future years. Despite the importance of this work arrangement for production and logistical demands, shift work significantly and negatively impacts the health of workers. Compared to daytime work, shift work and night work are recognized as risks factors for the development of cardiovascular diseases, metabolic diseases such as type-2 diabetes, cancer, and mental health problems, due to the misalignment of circadian rhythms, chronic sleep deprivation, and exposure to light at night $^{4.8}$. Moreover, the National Safety Council (2019) estimates that safety incidents are 30\% higher during night shifts. In addition, shift-workers with extended work periods $(>12 \mathrm{~h}$ ) and people with irregular shifts or fast rotating schedules have a higher risk of suffering accidents due to fatigue, which costs employers between USD $\$ 1,200$ and USD $\$ 3,100$ annually per employee in North America. As it is unlikely that the number of workers involved in atypical work schedules will decrease in the future, it is urgent to understand how shift work increases disease risk and to develop strategies that limit the adverse effects of working at times that are at odds with our biological time. The aim of this review is to provide an overview of the factors contributing to the detrimental effects of shift work on health and to highlight targeted interventions aimed at re-synchronizing the circadian clocks of those who need to remain on shift work schedules.

\section{NEGATIVE HEALTH IMPACT OF SHIFT WORK: CAUSES AND EFFECTS}

The three main factors that are thought to produce adverse effects on performance and health in shift workers are circadian misalignment, sleep deprivation, and exposure to light at night ${ }^{9}$. To better understand how shift work increases disease risk, it is necessary to first understand what circadian rhythms are and how they are regulated.

Circadian rhythms are near 24-hour oscillations, that regulate processes from gene expression to behavior ${ }^{4}$. Circadian rhythmicity is produced by a system consisting of a central pacemaker located in the brain and peripheral clocks located in different cells, tissues, and organs, which act in concert to produce daily rhythmicity. In mammals, circadian rhythmicity is orchestrated by the Suprachiasmatic Nucleus (SCN) of the hypothalamus, which acts as a "master peacemaker".
This peacemaker receives photic information from the retina through intrinsically photosensitive melanopsin containing ganglion cells (ipRGC) ${ }^{10}$. This signal synchronizes the SCN's network of neurons, and then transmits the rhythmic information to other brain areas and peripheral organs ${ }^{11,12}$. Clocks normally synchronize or entrain to the 24-hour light/dark cycle through environmental signals or zeitgebers. Light exposure is the strongest zeitgeber, but many social cues, such as meal times, school and work schedules, can also entrain the clock $^{13}$. Depending on an individual's internal time (given by the circadian clock) and their light environment, synchronization to the $24 \mathrm{~h}$ light/dark cycle can occur within a range of different relationships or phases of entrainment. These different relationships give rise to different cbronotypes, early (typically called "larks") or late (typically called "owls") depending, on when this happens within the light/dark cycle ${ }^{13}$.

\section{Circadian misalignment}

If the internal time of an individual and the timing of the physical (light/dark) and social (school or work time) environment are synchronized, it is said that they are aligned. On the other hand, circadian misalignment refers to the opposite situation, that is to say when the internal and external environment adopt an abnormal relative phase of entrainment ${ }^{12,14,15}$.

To determine the degree of circadian alignment, the relationship between the sleep/wake cycle and physiological parameters is normally measured. The time of lowest body temperature, of maximum and minimum levels of cortisol, and of the onset of melatonin increases measured under dim light conditions (DLMO), are usually used for this purpose ${ }^{15}$; pituitary hormones such as the thyroid stimulating hormone (TSH) and prolactin, known to possess a circadian component, have also been useful for this purpose. Among shift workers, a lack of entrainment of cortisol rhythms under a night schedule has been reported ${ }^{16}$. In addition, different measurements of anomalous cortisol levels have been described for night shift workers, although the results are inconclusive among different studies. For example, one investigation found high cortisol levels during daytime sleep in chronic night shift workers, in contrast to what occurs during the normal nighttime sleep of daytime workers. Moreover, night shift workers showed lower levels of cortisol and transient high levels of prolactin during work time, which contrasts with what is observed during the night in daytime workers ${ }^{16}$. On the other hand, using simulated laboratory conditions, another investigation found lower cortisol levels regardless of the state of alertness ${ }^{17}$. Inconclusive results of anomalous cortisol levels have also been described for early shift workers. For example, a Brazilian study conducted in male shift workers, showed that those working on early morning shifts (from 6 am to $2 \mathrm{pm}$ ) had higher levels of cortisol during a $24 \mathrm{~h}$ period when compared to night shift and daytime workers ${ }^{18}$. In contrast, one Argentinian study described a flattening in the morning to evening difference of cortisol levels for early shift male workers (with a mixed range of schedules, starting from 5:30 am to 8:30 am) in comparison with the differences observed in evening shift workers ${ }^{19}$. 
They could also be attributed to the experimental design, which avoided fast changes in sleep/wake schedules. Moreover, these inconclusive results could also be attributed to differences in shift length, shift schedules and the numbers of days off before the measurements were taken, which differs between studies. TSH hormone titer is also affected by shift work. Indeed, a retrospective study evaluated the levels of TSH in employees from a hospital in Korea and found that, compared to non-shift work, night shifts were associated with increased TSH levels and an increased risk of subclinical hypothyroidism among female workers ${ }^{20}$.

Food is another strong zeitgeber for peripheral organs and the timing of food intake can disrupt the coordinated clock system with adverse effects on energy metabolism ${ }^{21}$. During night shifts, individuals are active during the normally inactive rest phase, and work is commonly accompanied by mistimed food intake. Epidemiological studies reveal that shift work, especially night work, is a risk factor for type 2 diabetes. A large study that included 272.214 participants from the UK, examined the association of current and lifetime night shift work and the risk of developing type 2 diabetes. Their findings show that rotating night shift workers were more prone to develop type 2 diabetes in comparison to daytime workers, with the risk being even higher if they frequently performed night shifts within a given month ${ }^{22}$. Circadian misalignment has been proposed as possible explanation for this increased risk ${ }^{6}$. Glucose metabolism exhibits a diurnal pattern, with lower levels of postprandial glucose tolerance occurring after breakfast and diminished $\beta$-cell responsiveness and insulin action occurring after lunch and dinner in healthy humans ${ }^{23}$. Yet, after simulated night shift conditions, individuals exhibit lower glucose tolerance and a decrease insulin sensitivity after a meal ${ }^{24,25}$, two strong determinants of type 2 diabetes.

Chronic circadian misalignment has also been proposed as an underlying cause for the development of cardiovascular diseases (CVD) in shift workers. Indeed, under laboratory simulated conditions, one study found higher levels in mean arterial blood pressure after circadian misalignment ${ }^{26}$. A Brazilian cross-sectional study reported that, compared to daytime nurses, night shift nurses had a higher likelihood of reporting self-perceived hypertension, using the self-reported physician diagnosis of hypertension based on the question "Has a doctor ever told you that your blood pressure is or was high?" 27 . Moreover, a growing body of studies had associated shift work with an elevated risk of CVD, with the risk increasing the longer the person worked in shift work. For example, a meta-analysis found that among shift workers the risk of any CVD was $17 \%$ compared to that for daytime workers; furthermore after the first year of shift work, the risk of a CVD event increased $7.1 \%$ for every additional five years of shift work ${ }^{8}$. Likewise, a prospective cohort study that analyzed data from the National Health Studies I and II showed similar results. They found that a history of five or more years of shift work was associated with an increased risk of coronary heart disease, although the absolute increase was small ${ }^{28}$.
Taken together these results show that night shift workers experience a deep and complex state of desynchrony because they are constantly exposed to external synchronizers during the "wrong phases". Therefore, it is important to develop interventions aimed at re-aligning their internal and external environments in order to improve their health. Furthermore, additional interventions must be taken into account to limit the development of metabolic and cardiovascular diseases. These efforts should include the promotion of a healthy body weight and diet, no smoking and physical activity, among these workers ${ }^{29}$.

\section{Sleep Deprivation}

As mentioned above, shift workers, especially night workers, experience misalignments between their internal clocks and their sleep/wake schedules, including the REM and REM/non-REM segments of sleep, resulting in reduced sleep quality and duration ${ }^{30}$. As a consequence, when workers attempt to sleep and work out of phase with their internal rhythms, they commonly experience acutely disrupted daytime sleep and insomnia when they try to sleep at home, as well as excessive sleepiness at work ${ }^{31,32}$. A phone-conducted survey assessed the effects of work arrangements on sleep duration and excessive sleepiness in 3.345 New York citizens. This study found that night workers and rotating shift workers (morning to evening and to night shifts) are most likely to sleep less than 6 hours. They also reported higher rates of sleepiness at work compared to daytime workers ${ }^{32}$. Another study conducted on 5400 Norwegian nurses found that those with current or previous night work history experienced more cases of insomnia than did nurses with no night work experience ${ }^{33}$. In addition, a Korean study found that among 660 nurses, 33\% presented clinically significant insomnia and $46 \%$ presented sub-threshold insomnia. They also reported that insomnia was positively correlated with the number of night shift episodes per month ${ }^{34}$. A study conducted on a sample of shift workers from Southern Brazil identified a significant association between night shift work and sleep-related problems. Night shift workers reported poor sleep quality when compared to daytime workers, which was also associated with poor mental health ${ }^{35}$. Likewise, a Chilean study suggested that in comparison to daytime work, shift work worsened sleep quality and increased anxiety and depression ${ }^{36}$.

Although most night shift workers experience some degree of sleep desynchrony, misalignment is usually temporary, with recovery occurring within a few days after the return to a normal sleep/wake schedule. However, for some workers, shift work impairs their ability to sleep, resulting in a more prolonged recovery period, which is known as shift work sleep disorder (SWSD). According with the International Classification of Sleep Disorders: Diagnostic and Coding Manual (2001), this disorder includes "symptoms of insomnia and/or excessive sleepiness temporally associated with work schedules resulting in a reduction in total sleep time, unsatisfactory sleep or impaired alertness over the course of at least one month"31,37,38. In this regard, a study determined the prevalence associated with SWSD in a working population in Detroit. Approximately 10\% of the 2.570 workers interviewed, whose age ranged between 18- and 65-years old, experienced sleep disturbances and levels of sleepiness severe enough to meet the criteria of SWSD. 
These workers also had significantly higher rates of gastric ulcers, sleepiness-related accidents, absenteeism, depression, and altered familiar relationships ${ }^{39}$. Similarly, a sample of Japanese nurses engaged in shift work showed a prevalence of $24,4 \%$ of SWSD. Consistent with what was observed in the Detroit sample, the nurses that met the criteria for SWSD were more prone to experience traffic accidents, procedural errors at work, and work injuries, compared to those without SWSD ${ }^{40}$. Moreover, in a sample of shift workers from four different hospitals, those who obtained a positive result in the sleep disorder screening questionnaire were associated with almost twice the incidence of adverse safety outcomes over the following six months ${ }^{41}$.

These findings show that, although shift work is a major risk factor for the development of many health problems, the presence of SWSD in some workers also exposes them to a higher health-related morbidity associated with their sleep-wake symptomatology. There is therefore a need for investigations specifically aimed at identifying the characteristics that make some workers more vulnerable than others to the adverse effects of shift work under similar work conditions and schedules. In addition, it may be advisable to conduct more screening to identify individuals vulnerable to adverse health and safety outcomes.

\section{Exposure to light at night}

In 2007, The International Agency for Research on Cancer (IARC), a multidisciplinary and specialized cancer research agency of the World Health Organization, classified shift work with circadian disruption as a "probable buman carcinogen" Since then, a large number of additional epidemiologic studies have reported an association between shift work with circadian misalignment and the development of breast, colorectal, and prostate cancer ${ }^{5}$. In 2019, this classification was revisited by the IARC and night shift work was again declared to be a probable carcinogen to humans ${ }^{5}$. The largest group of studies considered during this update is related to breast cancer and included the results from two large studies: a cohort study that included data from the Nurses' Health Study I and II $(n=78.516$ and $n=114.559$, respectively) and a case-control study of 6.093 breast cancer cases and 6.933 controls, which examined the association between rotating night work and breast cancer. Both studies found that long-term rotating night-shift work was positively associated with a higher risk of breast cancer and that this risk was elevated in pre-menopausal women ${ }^{43,44}$. The epidemiological evidence for the light at night hypothesis makes a strong connection between lower melatonin levels and the development of breast cancer ${ }^{45}$. Melatonin is a pineal hormone that exerts a cyto-protective role by regulating oxidative stress, apoptosis processes, and mitochondrial homeostasis ${ }^{46}$. This hormone is entrained to the 24-hour cycle and under normal conditions reaches high levels at night and is acutely suppressed by light. Thus, this hypothesis proposes that light at night causes melatonin suppression. This reduction in melatonin levels in turn causes a decrease in the levels of circulating estrogen, which slows down the development and turnover of breast epithelial stem cells, and could cause them to become cancerous ${ }^{47}$.
In support for this hypothesis, a study assessed correlations between average urinary melatonin and plasma steroid hormone levels found that night work within the last two weeks was associated with a $56 \%$ reduction of 6-sulfatoxymelatonin (aMT6s), which is the major urinary metabolite of melatonin. Likewise, aMT6s levels were inversely associated with the levels of estradiol ${ }^{48}$. Finally, one recent study measured urinary levels of aMT6s in rotating-shift and daytime nurses for 3 days and found that during night shift the levels of aMT6s were lower compared to those of day-shift workers ${ }^{49}$.

Chronic and acute exposure to light at night has also been linked to abnormal mood and to deficits in cognitive function in shift workers and in rodent models ${ }^{4,33,50,51}$. In mice, two studies report how light can influence mood and learning through the circadian system. In the first study, the investigators found that light directly regulated mood related behaviors and cognitive functions through ipRGCs ${ }^{52}$. Years later another study showed that learning is regulated by ipRGCs that project to the SCN, but that this regulation was independent of the peacemaker function. They proposed that the effects of light on mood are mediated by ipRGCs that project to the perihabenular nucleus of the thalamus. This nucleus in turn projects to mood-regulating centers, completing the regulatory loop ${ }^{53}$. The understanding of the extent to which circadian rhythms are involved in normal physiological functioning and in pathological process will help to develop novel circadianbased interventions. Although the use of this approach is currently overlooked, in many cases its incorporation into classical clinical treatments has helped ease the symptoms even in cases where pharmacological approaches have not been successful ${ }^{5,55}$.

\section{FEATURES OF SHIFT WORK AND THEIR IMPACT IN THE HEALTH OF SHIFT WORKERS}

A wide number of sectors in our society have adopted shift work in order to provide $24 / 7$ services. In addition, a great diversity of shift working systems exists because every productive sector has adopted working schedules that may be preferred for their particular and complex organizational characteristics. However, the characteristics of these shift work systems, such as permanent night shifts, shift of different lengths, etc, combined with the individual differences in natural waking time may influence employee's performance and wellbeing. Here we discuss features of shift work that employers and employees should take into account in order to improve job performance and workers' wellbeing.

\section{Fixed v/s rotating shifts}

A long-standing debate in the field has been whether fixed or rotating shift schedules are preferable. During the initial years, the trend was to favor the use of fixed shift schedules claiming that permanent shifts were advantageous because they extended the duration of day sleep for night workers ${ }^{56}$. However, working several consecutive night shifts may simply increase sleep pressure and hence prolong daytime sleep ${ }^{57}$. 
More recently, studies assessing the degree of adjustment of endogenous rhythms and performance have brought into question the advantages of permanent night shifts. Indeed, current evidence suggest that less than $3 \%$ of permanent night workers present a correct phase in their endogenous melatonin rhythm and "less than one in four permanent night workers evidence sufficiently "substantial" adjustment to derive any benefit from it" ${ }^{58}$. With regard to permanent night workers' performance, recent studies show that cognitive performance is increasingly impaired as the night work schedule is carried out. Using a simulated night-shift protocol a study assessed how performance varies across consecutive night shifts, and found little evidence for performance adaptation during subsequent night shift periods ${ }^{59}$. In addition, a field study concluded that alertness and performance are impaired equally during the first night vs. subsequent nights among health workers $^{60}$. Moreover, another study showed that chronic shift workers under circadian misalignment show a deteriorated performance in tasks associated with sustained attention, information processing, and visual motor performance, when they were compared to daytime workers under the same protocol $^{51}$. Furthermore, a within-subject study in a sample of female health care workers in Germany observed a reduced psychomotor vigilance after a night shift in comparison to the results obtained after a day shift ${ }^{61}$. In summary, these results strongly suggest that fixed or permanent schedules should be avoided. It could also be said that complete adjustment (if there were any) may occur but only in workers who remain permanently under night shift schedules including during days off, which would be unusual given workers' social and domestic activities during their days off.

\section{Shift extension}

Among employers, especially from forestry, it is believed that extended working hours results in an increased production efficiency and harvesting, since it maximizes forest machinery use. In Chile, a leader in the forestry industry in South America, one study investigated the effects of extended shift hours and production. They found that even though production did increase as the working hours increased, the productivity per hour fell after 9 hour of shift length ${ }^{62}$, which contrasts with previous beliefs.

Twelve-hour shift systems have gained popularity among employees. They often report benefits such as having more free days, decreased number of work shift and shift changes ${ }^{63}$. In Finland, 599 industrial workers were exposed to three different shift schedules $[12 \mathrm{~h}$ fast rotating shifts (DDNN-----), $8 \mathrm{~h}$ fast rotating shifts (MMEENN----) and $8 \mathrm{~h}$ slow shifts (MMMMEEEENNNN-----) ( $\mathrm{D}=$ day, $\mathrm{N}=$ night, $\mathrm{M}=$ morning, $\mathrm{E}=$ evening,--=days off)] and were then consulted about their sleep quality and work satisfaction. Those engaged in $12 \mathrm{~h}$ fast rotating shift self-reported longer sleep duration, used less sleep promoting medication, and were more satisfied than their colleagues in 8 hour shifts ${ }^{64}$. Although $12 \mathrm{~h}$ fast rotating shift system seems to increase the self-satisfaction and self-perception of sleep, long work shift is consistently associated with negative effects.
For example, an association between the duration of the shift and disturbed sleep has been reported ${ }^{63,65}$ along with a higher risk of dementia ${ }^{66}$. Likewise, work periods of 12 hours carry twice the risk of accidents than do 8 -hour periods ${ }^{67}$. Importantly, in health professionals, the extended shift periods can negatively affect the health outcomes of patients. For example, a survey conducted in 12 European countries reported that nurses working $>12 \mathrm{~h}$ were more likely to provide reduced quality of care, left more work unfinished, their patients were more prone to suffer adverse events related to hospital interventions ${ }^{68}$, and tended to take longer to read vital signs ${ }^{69}$.

Whereas longer shifts are thought to be convenient for employees and employers because they compress workdays and use fewer staff resources, any benefit may be lost if the dangers for workers and customers is higher; cheaper can be expensive in the long run.

\section{Chronotype and work schedules}

Chronotype (i.e., when the person is naturally awake under a normal light:dark schedule) is another important feature that should be taken into account if any attempt to optimize work schedules is to be achieved. A field study that used modified surveys to assess sleep/wake behavior under different shift schedules (the Munich ChronoType Questionaire (MCTQ $\left.{ }^{\text {shift}}\right)^{13}$ and the psychomotor vigilance test), suggested that chronotype influences performance by interacting with sleep. Performance was affected by the time awake during night shifts and by the time awake during morning shift in a sample of workers classified as later chronotypes ${ }^{70}$. Using the same questionnaire other studies found that early chronotypes showed reduced sleep duration and higher levels of sleep disturbance during night shifts. The same results were found for later chronotypes for morning shifts, along with a higher need for recovery after morning shift periods, independent of age ${ }^{71,72}$. But chronotype may not only influence performance and sleep quality; it has also been associated with a higher risk of depression in middle- to older-aged women, according to a prospective analysis including 32.740 women from the Nurses’ Health Study $\mathrm{II}^{73}$. Moreover, the interaction between chronotype and work schedules is associated with an increased or reduced risk for type 2 diabetes for early and late chronotypes, respectively ${ }^{74}$.

Including chronotype in the design of shift schedules could represent an opportunity to obtain the best performance from workers, as it would tailor their work schedule to their individual characteristics. In this regard, one investigation designed a chronotype-adjusted (CTA) schedule. For this they used the MCTQ ${ }^{\text {shift }}$ to guide the suppression of those shifts that contributed to increased stress for extreme chronotypes (morning shifts for late chronotypes and night shifts for morning chronotypes). This intervention increased sleep duration and quality, reduced circadian misalignment, and produced an increase in perceived wellbeing ${ }^{75}$. Finally, it is important to mention that any intervention regarding chronotype should be reassessed periodically, since chronotype changes with age ${ }^{76}$. 
LIGHT-BASED INTERVENTIONS TO IMPROVE CIRCADIAN ADAPTATION TO SHIFT WORK

Our work-centered culture requires that individuals work under nonstandard schedules. To cope with this lifestyle, many depend on a stimulant/sedative loop. Naps and stimulants such as caffeine and modafinil are used to increase alertness during night shifts, but they do not restore alertness and performance to daytime levels ${ }^{77,78}$. Moreover, sedative medication is used to increase daytime sleep levels but is ineffective against the nadir in alertness and performance that occurs during night work ${ }^{51,60}$. By contrast, interventions that phase shift the circadian clock to re-align the physiology to night work can be a more useful measure to counter the adverse effects of night work.

\section{Beyond caffeine: Light based interventions}

The aim of the light-based interventions is to produce a gradual phase delay of the worker's circadian rhythm, based on measurements of their minimum temperature $\left(T_{\min }\right)$, which is considered to be the period with the lowest alertness. Thus, such interventions push $\mathrm{T}_{\min }$ to the daytime period and therefore, move the period of alertness into the night ${ }^{79}$. As light is the most powerful zeitgeber ${ }^{13}$, scheduled exposure to bright light before the $\mathrm{T}_{\min }$ and scheduled dark/sleep episodes during the following day to avoid an advancing of the circadian clock, have been used for this purpose in field and in laboratory studies. In this regard different experimental designs have been used. Using a combination of dark environment, sunglasses (SG) for the commute home, melatonin $(\mathrm{M})$, and a moving pattern of bright light pulses (BL) ( 5000 lux/20 min) one study demonstrated that BL was the most effective measure to phase delay DLMO into daytime sleep ${ }^{79}$. Another investigation combining SG, a static pattern of BL, and exposure to sunlight after daytime sleep in simulated night shift workers, found the same results ${ }^{80}$. In a field study nurses exposed intermittently to bright light during the first 6 hours of their night shifts in combination with SG were able to delay their DLMO into the day dark/sleep episodes and increase the length of daytime sleep, even when the length of exposure to intermittent BL during work was not evenly controlled for every nurse ${ }^{81}$. Using the same approach, one study aligned the peak of cortisol with night work and the nadir with daytime sleep ${ }^{82}$. Phase shifting circadian rhythms to completely align to night work and daytime sleep is the major goal of all the interventions described. However, these approaches imply that people sleep during the day and remain awake during the night in a permanent manner, which is not useful for workers under rotating schedules, since they would then need to spend part of their days off re-synchronizing their clocks to a daytime regime. For this reason, investigators have developed new strategies that produce a compromise phase position in which the circadian clock is delayed to only partially align with the day sleep period.
This partial entrainment is designed to attenuate the fall in performance and alertness that would normally occur during night shifts while permitting sufficient daytime sleep after night shifts ${ }^{83}$. Using a combination of brief bright light pulses during night shifts ( 4100 lux/15 min every hour from midnight until 4 am), sunglasses, scheduled sleep in dark bedrooms as soon as possible after the end of the shift, and exposure to sunlight by the afternoon, a series of studies reported delayed DLMO to the compromise phase position, larger daytime sleep, and improved performance and alertness ${ }^{83-85}$. Although this arrangement was designed for permanent night workers, the same elements but in a more complex combination have also been used to help rotating shift workers ${ }^{86}$.

In summary, even when these recommendations have been available almost for two decades, they are underused in the real world, despite their low economical cost. The most common complaint for these treatments is that they are time consuming, which could impact the compliance with the treatment ${ }^{87}$. Thus, the obvious challenge is to develop portable devices that allow workers to self-administer pulses of BL while on duty. Indeed, even when these devices exist (https://sadlampsusa.com/ best-light-therapy-glasses/) we are still far from the target. It is necessary to continue improving the quality of theses glasses and make them more "job friendly" and economically accessible than they currently are.

\section{CONCLUSION}

Global modern economy depends nowadays on nontraditional work arrangements. However, working against our body clock is strongly associated with a broad range of chronic diseases. It is not by chance that the rapid increase in diseases such type- 2 diabetes, cancer, and neurological disorders, parallels the epidemic of circadian disruption. As was reviewed here, the negative effects on the health of an increasing proportion of the work force are indeed due to circadian misalignment and exposure to light at odd hours. Hopefully, our knowledge in the field of circadian rhythms is increasingly being translated into measures that improve human health ${ }^{88}$. This knowledge should be oriented to the development of strategies that allow public health workers, employers, governments, and any other affected stakeholder in our society, to deal most effectively with the consequences of our $24 / 7$ culture.

\section{ACKNOWLEDGEMENTS}

Grateful thanks are due to the organizing committee of the XV Latin American Symposium on Chronobiology who awarded me the Sleep Science prize based on my presentation. My thanks are also due to John Ewer, who reviewed earlier drafts of this manuscript and contributed to its improvement. 


\section{REFERENCES}

1. Foster RG, Wulff K. The rhythm of rest and excess. Nat Rev Neurosci. 2005;6(5):407-14.

2. Eurofound. Fifth European working conditions survey. 2012. 151 p.

3. Maestas N, Mullen K, Powell D, von Wachter T, Wenger J. Working Conditions in the United States. Rand. 2015;

4. Logan RW, McClung CA. Rhythms of life: circadian disruption and brain disorders across the lifespan. Nat Rev Neurosci. 2019;20(1):49-65.

5. Ward EM, Germolec D, Kogevinas M, McCormick D, Vermeulen R, Anisimov VN, et al. Carcinogenicity of night shift work. Lancet Oncol [Internet]. 2019;1-2. Available from: https://linkinghub.elsevier.com/ retrieve/pii/S1470204519304553

6. Kolbe I, Oster H. and the Regulation of Inflammation in Adipose Tissues. Yalle J Biol Med. 2019;92:317-25.

7. Gan Y, Yang C, Tong X, Sun H, Cong Y, Yin X, et al. Shift work and diabetes mellitus: A meta-analysis of observational studies. Occup Environ Med. 2015;72(1):72-8.

8. Torquati L, Mielke GI, Brown WJ, Kolbe-Alexander T. Shift work and the risk of cardiovascular disease. A systematic review and meta-analysis including dose-response relationship. Scand J Work Environ Heal. 2018;44(3):229-38.

9. Evans JA, Davidson AJ. Health consequences of circadian disruption in humans and animal models [Internet]. 1st ed. Vol. 119, Progress in Molecular Biology and Translational Science. Elsevier Inc.; 2013. 283323 p. Available from: http://dx.doi.org/10.1016/B978-0-12-3969712.00010-5

10. Hattar S, Lucas R, Mrosovsky N, Thompson S, Douglas R, Hankis M, et al. Melanopsin and rod-cone photoreceptive systems account for all major accessory visual functions in mice. Nature. 2003;424(6944):76-81.

11. Dibner C, Schibler U, Albrecht U. The Mammalian Circadian Timing System: Organization and Coordination of Central and Peripheral Clocks. Vol. 72, Annual Review of Physiology. 2010. 517-549 p.

12. Roenneberg T, Merrow M. The circadian clock and human health. Curr Biol [Internet]. 2016;26(10):R432-43. Available from: http://dx.doi org/10.1016/j.cub.2016.04.011

13. Roenneberg T, Kumar CJ, Merrow M. The human circadian clock entrains to sun time. 2007;17(2):4-6.

14. Vetter C. Circadian disruption: What do we actually mean? Eur J Neurosci. 2018;

15. Baron KG, Reid KJ. Circadian misalignment and health. Int Rev Psychiatry. 2014;26(2):139-54.

16. Weibel L, Branderberger G. Disturbances in Hormonal Profiles of Night Workers during Their Usual Sleep and Work Times. J Biol Rhythms. 1998;13(3):202-8.

17. Wright KP, Drake AL, Frey DJ, Fleshner M, Desouza CA, Gronfier C, et al. Influence of sleep deprivation and circadian misalignment on cortisol, inflammatory markers, and cytokine balance. Brain Behav Immun [Internet]. 2015;47(January):24-34. Available from: http://dx.doi org/10.1016/j.bbi.2015.01.004

18. Padilha HG, Crispim CA, Zimberg IZ, Folkard S, Tufik S, De Mello MT. Metabolic responses on the early shift. Chronobiol Int. 2010;27(5):1080-92.

19. Diez JJ, Vigo DE, Lloret SP, Rigters S, Role N, Cardinali DP, et al. Sleep habits, alertness, cortisol levels, and cardiac autonomic activity in shortdistance bus drivers: Differences between morning and afternoon shifts. J Occup Environ Med. 2011;53(7):806-11.

20. Moon SH, Lee BJ, Kim SJ, Kim HC. Relationship between thyroid stimulating hormone and night shift work. Ann Occup Environ Med [nternet]. 2016;28(1):1-5. Available from: http://dx.doi.org/10.1186/ s40557-016-0141-0

21. Hatori M, Vollmers C, Zarrinpar A, DiTacchio L, Bushong EA, Gill S, et al. Time-restricted feeding without reducing caloric intake prevents metabolic diseases in mice fed a high-fat diet. Cell Metab. 2012;15(6):848-60

22. Vetter C, Dashti HS, Lane JM, Anderson SG, Schernhammer ES, Rutter MK, et al. Night shift work, genetic risk, and type 2 diabetes in the UK biobank. Diabetes Care. 2018;41(4):762-9.

23. Saad A, Man CD, Nandy DK, Levine JA, Bharucha AE, Rizza RA, et al. Diurnal pattern to insulin secretion and insulin action in healthy individuals. Diabetes. 2012;61(11):2691-700.

24. Morris CJ, Purvis TE, Mistretta J, Scheer FAJL. Effects of the internal circadian system and circadian misalignment on glucose tolerance in chronic shift workers. J Clin Endocrinol Metab. 2016;101(3):1066-74.

25. Qian J, Dalla Man C, Morris CJ, Cobelli C, Scheer FAJL. Differential effects of the circadian system and circadian misalignment on insulin sensitivity and insulin secretion in humans. Diabetes, Obes Metab. 2018;20(10):2481-5.
26. Scheer FAJL, Hilton MF, Mantzoros CS, Shea SA. Adverse metabolic and cardiovascular consequences of circadian misalignment. Proc Natl Acad Sci U S A. 2009;106(11):4453-8.

27. Rotenberg L, Silva-Costa A, Vasconcellos-Silva PR, Griep RH. Work schedule and self-reported hypertension - the potential beneficial role of on-shift naps for night workers. Chronobiol Int. 2016;33(6):697-705.

28. Vetter C, Devore EE, Wegrzyn LR, Massa J, Speizer FE, Kawachi I, et al. Association between rotating night shiftwork and risk of coronary heart disease among women. JAMA - J Am Med Assoc. 2016;315(16):1726-34.

29. Vetter C, Scheer FAJL. A healthy lifestyle — reducing T2DM risk in shift workers? Nat Rev Endocrinol. 2019;15(4):194-6.

30. Boivin DB, Boudreau P. Impacts of shift work on sleep and circadian rhythms. Pathol Biol [Internet]. 2014;62(5):292-301. Available from: http://dx.doi.org/10.1016/j.patbio.2014.08.001

31. Cheng P, Drake C. Shift Work Disorder. Neurol Clin [Internet]. 2019;37(3):563-77. Available from: https://doi.org/10.1016/j. ncl.2019.03.003

32. Ohayon, M; Smolensky, M; Roht T. Consequences of Shiftworking on Sleep Duration, Sleepiness, and Sleep Attacks. Chronobiol Int. 2010;27(3):575-89.

33. Øyane NMF, Pallesen S, Moen BE, Åkerstedt T, Bjorvatn B. Associations Between Night Work and Anxiety, Depression, Insomnia, Sleepiness and Fatigue in a Sample of Norwegian Nurses. PLoS One. 2013;8(8):1-7.

34. Jung HS, Lee B. Contributors to shift work tolerance in South Korean nurses working rotating shift. Appl Nurs Res. 2015;28(2):150-5.

35. Olinto MTA, Garcez A, Henn RL, Macagnan JBA, Paniz VMV, Pattussi MP. Sleep-related problems and minor psychiatric disorders among Brazilian shift workers. Psychiatry Res [Internet]. 2017:257:412-7. Available from: http://dx.doi.org/10.1016/j.psychres.2017.08.018

36. Carrillo J, Becerra M. Anxiety and depression associated with sleep disturbances in shift workers. Sleep Med. 2013;14(2013):e91.

37. ICSD. The International Clasification of Sleep Disorders, Revised American Academy of Sleep Medicine. International Classification. 2001.

38. Booker LA, Magee M, Rajaratnam SMW, Sletten TL, Howard ME. Individual vulnerability to insomnia, excessive sleepiness and shift work disorder amongst healthcare shift workers. A systematic review. Sleep Med Rev [Internet]. 2018;41:220-33. Available from: https://doi. org/10.1016/j.smrv.2018.03.005

39. Drake C, Roehrs T, Richardson T, Walsh J, Roth T. Shift work sleep disorder: Prevalence and consequences beyond that of symptomatic day workers. Sleep [Internet]. 2004;27(8):1453-62. Available from: http:// ovidsp.ovid.com/ovidweb.coi? $\mathrm{T}=\mathrm{JS} \& \mathrm{PAGE}=$ reference $\& \mathrm{D}=$ emed6\&N EWS $=\mathrm{N} \& A \mathrm{~N}=2005013075$

40. Asaoka S, Aritake S, Komada Y, Ozaki A, Odagiri Y, Inoue S, et al Factors associated with shift work disorder in nurses working with rapidrotation schedules in Japan: The nurses' sleep health project. Chronobiol Int. 2013;30(4):628-36.

41. Weaver MD, Vetter C, Rajaratnam SMW, O’Brien CS, Qadri S, Benca RM, et al. Sleep disorders, depression and anxiety are associated with adverse safety outcomes in healthcare workers: A prospective cohort study. J Sleep Res. 2018;27(6):1-9.

42. Straif K, Baan R, Grosse Y, Secretan B, Ghissassi F El, Bouvard V, et al Carcinogenicity of shift-work, painting, and fi re-fi ghting. 2008;1065-6.

43. Wegrzyn LR, Tamimi RM, Rosner BA, Brown SB, Stevens RG, Eliassen $\mathrm{AH}$, et al. Rotating night-shift work and the risk of breast Cancer in the nurses' health studies. Am J Epidemiol. 2017;186(5):532-40.

44. Cordina-Duverger E, Menegaux F, Popa A, Rabstein S, Harth V, Pesch B, et al. Night shift work and breast cancer: a pooled analysis of populationbased case-control studies with complete work history. Eur J Epidemiol. 2018;33(4):369-79.

45. Hill SM, Belancio VP, Dauchy RT, Xiang S, Brimer S, Mao L, et al. Melatonin: An inhibitor of breast cancer. Endocr Relat Cancer. 2015;22(3):R183-204

46. Carrillo-vico A, Lardone PJ, Álvarez-sánchez N. Melatonin : Buffering the Immune System. 2013;8638-83.

47. Hunter CM, Figueiro MG. Measuring Light at Night and Melatonin Levels in Shift Workers: A Review of the Literature. Biol Res Nurs. 2017;19(4):365-74.

48. Stevens RG, Schernhammer E. Epidemiology of urinary melatonin in women and its relation to other hormones and night work. SO - Cancer Epidemiol Biomarkers Prev 2005 Feb;14(2):551; author reply 551. Cancer Epidemiol Biomarkers Prev. 2005;14(2):551; author reply 551551; author reply 551 .

49. Razavi P, Devore EE, Bajaj A, Lockley SW, Figueiro MG, Ricchiuti V, et al. Shift Work, Chronotype, and Melatonin Rhythm in Nurses. Cancer Epidemiol Biomarkers Prev [Internet]. 2019;cebp.1018.2018. Available from: http://cebp.aacrjournals.org/lookup/doi/10.1158/1055-9965.EPI-18-1018 
50. Walker WH, Borniger JC, Gaudier-Diaz MM, Hecmarie MeléndezFernández O, Pascoe JL, Courtney DeVries A, et al. Acute exposure to low-level light at night is sufficient to induce neurological changes and depressive-like behavior. Mol Psychiatry. 2019;

51. Chellappa SL, Morris CJ, Scheer FAJL. Effects of circadian misalignment on cognition in chronic shift workers. Sci Rep [Internet]. 2019;9(1):1-9. Available from: http://dx.doi.org/10.1038/s41598-018-36762-w

52. Legates TA, Altimus CM, Wang H, Lee HK, Yang S, Zhao H, et al. Aberrant light directly impairs mood and learning through melanopsinexpressing neurons. Nature. 2012;491(7425):594-8.

53. Fernandez DC, Fogerson PM, Lazzerini Ospri L, Thomsen MB, Layne RM, Severin D, et al. Light Affects Mood and Learning through Distinct Retina-Brain Pathways. Cell [Internet]. 2018;175(1):71-84.e18. Available from: https://doi.org/10.1016/j.cell.2018.08.004

54. Wu JC, Kelsoe JR, Schachat C, Bunney BG, DeModena A, Golshan S, et al. Rapid and Sustained Antidepressant Response with Sleep Deprivation and Chronotherapy in Bipolar Disorder. Biol Psychiatry. 2009;66(3):298301.

55. Sikkens D, Riemersma - Van der Lek RF, Meesters Y, Schoevers RA, Haarman BCM. Combined sleep deprivation and light therapy: Clinical treatment outcomes in patients with complex unipolar and bipolar depression. J Affect Disord [Internet]. 2019;246(November 2018):72730. Available from: https://doi.org/10.1016/j.jad.2018.12.117

56. Wilkinson RT. How fast should the night shift rotate? Ergonomics. 1992;35(12):1425-46.

57. Pilcher JJ, Lambert BJ, Huffcutt AI. Differential Effects of Permanent and Rotating Shifts on Self-Report Sleep Length: A Meta-Analytic Review. Sleep [nternet]. 2000;23(2):1-9. Available from: https://academic.oup. $\mathrm{com} / \mathrm{sleep} /$ article-lookup/doi/10.1093/sleep/23.2.1b

58. Folkard S. Do permanent night workers show circadian adjustment? A review based on the endogenous melatonin rhythm. Chronobiol Int. 2008;25(2-3):215-24.

59. McHill AW, Wright KP. Cognitive Impairments during the Transition to Working at Night and on Subsequent Night Shifts. J Biol Rhythms. 2019;1-15.

60. Ganesan S, Magee M, Stone JE, Mulhall MD, Collins A, Howard ME, et al. The Impact of Shift Work on Sleep, Alertness and Performance in Healthcare Workers. Sci Rep. 2019;9(1):1-13.

61. Behrens T, Burek K, Pallapies D, Kösters L, Lehnert M, Beine A, et al. Decreased psychomotor vigilance of female shift workers after working night shifts. PLoS One. 2019;14(7):1-17.

62. Pasicott P, Murphy GE. Effect of work schedule design on productivity of mechanised harvesting operations in Chile. New Zeal J For Sci. 2013;43(Celone 2007):1-10.

63. Sallinen M, Kecklund G. Shift work, sleep, and sleepiness - Differences between shift schedules and systems. Scand J Work Environ Heal. 2010;36(2):121-33.

64. Karhula K, Härmä M, Ropponen A, Hakola T, Sallinen M, Puttonen S. Sleep and satisfaction in 8- and 12 -h forward-rotating shift systems: Industrial employees prefer 12-h shifts. Chronobiol Int. 2016;33(6):768-75.

65. Iskera-golec I, Folkard S. Work \& Stress : An International Journal of Work, Health \& Organisations Health, well-being and burnout of ICU nurses on 12- and 8-h shifts Health, well-being and burnout of ICU nurses on 12- and 8-h shifts. Work Stress. 1996; (June 2013):37-41.

66. Nabe-Nielsen K, Hansen ÅM, Ishtiak-Ahmed K, Grynderup MB, Gyntelberg F, Islamoska S, et al. Night shift work, long working hours and dementia: A longitudinal study of the Danish Work Environment Cohort Study. BMJ Open. 2019;9(5):1-9.

67. Wagstaff AS, Lie JAS. Shift and night work and long working hours - a systematic review of safety implications. Scand J Work Environ Heal. 2011;37(3):173-85.

68. Griffiths P, Dall'Ora C, Simon M, Ball J, Lindqvist R, Rafferty A-M, et al. Nurses' Shift Length and Overtime Working in 12 European Countries. Med Care. 2014;52(11):975-81.
69. Dall'ora C, Griffiths P, Redfern O, Recio-Saucedo A, Meredith P, Ball J. Nurses' 12-hour shifts and missed or delayed vital signs observations on hospital wards: Retrospective observational study. BMJ Open. 2019;9(1):1-6.

70. Vetter C, Juda M, Roenneberg T. The influence of internal time, time awake, and sleep duration on cognitive performance in shiftworkers. Chronobiol Int. 2012;29(8):1127-38.

71. Juda M, Vetter C, Roenneberg T. Chronotype modulates sleep duration, sleep quality, and social jet lag in shift-workers. J Biol Rhythms. 2013;28(2):141-51.

72. van de Ven HA, van der Klink JJL, Vetter C, Roenneberg T, Gordijn M, Koolhaas W, et al. Sleep and need for recovery in shift workers: do chronotype and age matter? Ergonomics [Internet]. 2016;59(2):310-24. Available from: http://dx.doi.org/10.1080/00140139.2015.1058426

73. Vetter C, Chang SC, Devore EE, Rohrer F, Okereke OI, Schernhammer ES. Prospective study of chronotype and incident depression among middle- and older-aged women in the Nurses' Health Study II. J Psychiatr Res [Internet]. 2018;103:156-60. Available from: https://doi. org/10.1016/j.jpsychires.2018.05.022

74. Vetter C, Devore EE, Ramin CA, Speizer FE, Willett WC, Schernhammer ES. Mismatch of sleep and work timing and risk of type 2 diabetes. Diabetes Care. 2015;38(9):1707-13.

75. Vetter C, Fischer D, Matera JL, Roenneberg T. Aligning work and circadian time in shift workers improves sleep and reduces circadian disruption. Curr Biol. 2015;25(7):907-11.

76. Foster RG, Roenneberg T. Human Responses to the Geophysical Daily, Annual and Lunar Cycles. Curr Biol. 2008;18(17):784-94.

77. Czeisler CA, Walsh JK, Roth T, Hughes RJ, Wright KP, Kingsbury L, et al. Modafinil for Excessive Sleepiness Associated with Shift-Work Sleep Disorder. N Engl J Med. 2005;353(5):476-86.

78. Schweitzer PK, Randazzo AC, Stone K, Erman M, Walsh JK. Laboratory and field studies of naps and caffeine as practical countermeasures for sleep-wake problems associated with night work. Sleep [Internet] 2006;29(1):39-50. Available from: http://www.embase.com/search/res ults? subaction $=$ viewrecord $\&$ from $=$ export\&id $=\mathrm{L} 44019029 \% 0 \mathrm{Ahttp}: / /$ dx.doi.org/10.1093/sleep/29.1.39

79. Crowley SJ, Lee C, Tseng CY, Fogg LF, Eastman CI. Combinations of Bright Light, Scheduled Dark, Sunglasses, and Melatonin to Facilitate Circadian Entrainment to Night Shift Work. J Biol Rhythms. 2003;18(6):513-23.

80. Lee C, Smith M, Eastman C. A compromise phase position for permanent night shift workers: Circadian phase after two night shifts with scheduled sleep and light/dark exposure. Chronobiol Int. 2006;23(4):859-75.

81. Boivin DB, Boudreau P, James FO, Kin NMKNY. Photic resetting in nightshift work: Impact on nurses' sleep. Chronobiol Int. 2012;29(5):619-28.

82. James FO, Walker CD, Boivin DB. Controlled exposure to light and darkness realigns the salivary cortisol rhythm in night shift workers. Chronobiol Int. 2004;21(6):961-72.

83. Smith MR, Eastman CI. Night Shift Performance is Improved by a Compromise Circadian Phase Position. Sleep. 2008;31(12):1639-45.

84. Smith MR, Fogg LF, Eastman CI. Practical interventions to promote circadian adaptation to permanent night shift work: Study 4. J Biol Rhythms. 2009;24(2):161-72.

85. Smith MR, Fogg LF, Eastman CI. A compromise circadian phase position for permanent night work improves mood, fatigue, and performance. Sleep. 2009;32(11):1481-9.

86. Eastman C. How to reduce circadian misalignment in rotating shift workers. ChronoPhysiology Ther. 2016;Volume 6:41-6.

87. Crowley SJ, Eastman CI. Phase advancing human circadian rhythms with morning bright light, afternoon melatonin, and gradually shifted sleep: CAN we reduce morning bright-light duration? Sleep Med [Internet] 2015;16(2):288-97. Available from: http://dx.doi.org/10.1016/j. sleep.2014.12.004

88. Panda S. The arrival of circadian medicine. Year Rev. 2019;63(3-4):67-9. 


\section{The impact of training shifts in dancers' chronotype and sleep patterns}

Natalia Coirolo ${ }^{1,2}$

Ana Silva ${ }^{1}$

Bettina Tassino ${ }^{2 *}$

\footnotetext{
${ }^{1}$ Laboratorio de Neurociencias, Facultad de Ciencias, Universidad de la República, Montevideo, Uruguay.

${ }^{2}$ Sección Etología, Facultad de Ciencias, Universidad de la República, Montevideo, Uruguay.
}

\begin{abstract}
Circadian preferences (chronotypes) as well as human sleep patterns depend on internal and environmental factors including social demands. School and work shifts are advantageous tools for studying the way social pressures impact on the biological clock. We took advantage of the Uruguayan public professional training in dance organized in two different shifts (morning, 8:30 to 12:30, and night, 20:00 to 24:00) to evaluate the influence of shifts on sleep timing and individual circadian preferences of dancing trainees $(n=56)$ from data obtained by questionnaires (Munich Chronotype Questionnaire, MCTQ, and Morningness-Eveningness Questionnaire, MEQ) and sleep logs (SL). Although the outputs of MEQ and MCTQ significantly correlated, nocturnal dancers reported later chronotypes (measured by MCTQ) than morning dancers, but no differences in their circadian preferences measured by MEQ. Both MCTQ and SL showed that nocturnal dancers scheduled their sleep significantly later than morning ones during work and free days.
\end{abstract}

Keywords: Chronotypes; Sleep Patterns; Circadian Preferences; Questionnaires; Sleep Logs Training Shift 


\section{INTRODUCTION}

In humans, the intrinsic period of the circadian cycle is slightly greater than $24 \mathrm{~h}$ on average with individual variations that depend on both genetic and environmental factors ${ }^{1}$. Individual differences in the phase of the circadian rhythms are known as circadian preferences or chronotypes ${ }^{1}$. Chronotypes depend on the expression of several genes ${ }^{2}$, and vary with other biological factors such as age and $\operatorname{sex}^{3}$. Chronotypes also depend on the intensity, quality and timing of light exposure ${ }^{4}$ and on a diversity of social demands such as school, work, or entertainment schedules ${ }^{5}$.

The sleep-wake cycle is the most conspicuous human circadian rhythm, which is well-known to depend on social demands. Latin American high school students attending school in the morning shift have advanced and shorter sleep compared to those attending the afternoon-shift ${ }^{6-9}$. Night shift work has also been associated to sleep disorders ${ }^{10}$. Shifts not only affect sleep timing but also circadian preferences. For example, nightshift nurses have significantly later chronotypes than day-shift nurses ${ }^{11}$. Moreover, afternoon-shift high school students from Mexico and Uruguay have significantly later chronotypes than morning-shift ones ${ }^{7,12}$

Individual circadian preferences can be inferred by universally validated questionnaires ${ }^{13,14}$. However, different questionnaires survey different aspects of sleep habits and might not be consistent in typifying chronotype. MCTQ, for example explores sleep timing and assumes that the mid sleep point on free days corrected for sleep debt on workdays (MSFsc) is a good proxy of individual chronotype ${ }^{3}$. MEQ score, in turn, represents the self-reported time preference to perform different activities ${ }^{13}$. Therefore, while MEQ and MSFsc usually correlate, it is not surprising to find discrepancies as both questionnaires have different aims and are not interchangeable. A more objective way of evaluating sleep habits is provided by sleep logs (SL), which despite being self-reported, are more accurate, providing information about actual daily sleep timing, which in turn, might be (or not) in accordance with circadian preferences ${ }^{15}$. An integration of all these instruments is required to have a reliable picture of individual sleep habits and circadian preferences of a given population.

In people with demanding physical or athletic training, sleep patterns and rest times as well as the time in which training is scheduled, are relevant to their performance.
Athletes with long training days, extended working periods, and irregular rest in weekends, frequently have impaired sleep duration and efficiency ${ }^{16}$. As a particular case, dancers are competitive athletes who undergo extreme physical and mental stress and usually work according to an irregular schedule. However, the relationship between circadian preferences, sleep patterns, and performance in dancers has not been thoroughly evaluated so far. To our knowledge, only one previous study reports the decrease in sleep quality and the cognitive impairment ballet dancers suffer during training ${ }^{17}$.

In this study, we took advantage of the Uruguayan public professional training in dance which is organized in two different shifts (morning and night). We aimed to evaluate the influence of these contrasting shifts on sleep timing and individual circadian preferences of dancing trainees from data obtained by questionnaires and SL. Both types of instruments showed that nocturnal dancers scheduled their sleep later than morning ones. In addition, nocturnal dancers reported later circadian chronotypes (measured by MSFsc) than morning dancers, with no differences in their circadian preferences measured by MEQ.

\section{MATERIAL AND METHODS}

Dancers from the Uruguayan public school for professional training in contemporary and folkloric dance (Escuela Nacional de Danza, END-SODRE, Ministerio de Educación y Cultura) were recruited to participate in this study (Table 1). To maximize school infrastructure usage, the END-SODRE is organized as a 4-year training program with classes taught from Monday through Friday in two shifts. First and second grade students attend the night shift (20:00 to 24:00) while students of the third and fourth grade attend the morning shift (8:30 to 12:30). Fiftysix dancers (29 from the morning shift and 27 from the night shift), mostly females, with age ranging from 18 to 30 years old met the inclusion criteria as participants of this study (Table 1). Dancers under self-reported treatment with psycho-active drugs, with missing data in questionnaires, and reporting the use of alarm clock during weekends were excluded from this study. Data were globally analyzed with no distinction among genders.

During August 2019, informational flyers and informed consent forms were distributed . Enrolled participants answered questionnaires during school-time. This study was evaluated by the Ethics Committee of the School of Psychology, Universidad de la República, and complied with the principles outlined by the Declaration of Helsinki (World Medical Association, 2013).

Table 1. Number of participants, gender, age and chronobiological characterization of the dancers training in morning-shift and night-shift.

\begin{tabular}{|c|c|c|c|c|c|}
\hline & & Total & Morning-shift & Night-shift & \\
\hline & & & & & $\mathrm{p}$ \\
\hline Participants (n) & & 56 & 29 & 27 & \\
\hline \multirow[t]{3}{*}{ Gender (n) } & Female & 45 & 24 & 21 & \\
\hline & Male & 7 & 3 & 4 & \\
\hline & Other & 4 & 2 & 2 & \\
\hline Age (mean $\pm \mathrm{SD})$ & & $22.07 \pm 2.49$ & $22.55 \pm 2.69$ & $21.56 \pm 2.19$ & 0.1785 \\
\hline MSFsc (mean $\pm S D$ ) & & $6: 10 \pm 1: 52$ & $5: 43 \pm 1: 47$ & $6: 40 \pm 1: 52$ & 0.0472 \\
\hline MEQ score (mean $\pm \mathrm{SD})$ & & $46.91 \pm 8,88$ & $48.83 \pm 8.51$ & $44.85 \pm 8.96$ & 0.1092 \\
\hline
\end{tabular}


The chronobiological characterization was assessed using the Spanish version of both the Munich Chronotype Questionnaire (MCTQ, ${ }^{14}$ and the Morningness-Eveningness (MEQ, $\left.{ }^{13}\right)$. Validated MCTQ reports were used to assess the mid-sleep point on free days corrected for sleep debt on workdays (MSFsc) as a proxy of individual chronotype ${ }^{3}$, and the social jetlag as the absolute difference between the midpoints of sleep on work and free days ${ }^{5}$. The MEQ score, calculated from the answers about preferred sleep time and daily performance inquired in the MEQ, was also considered as a proxy for individual circadian preference, with higher scores indicating greater morningness tendencies ${ }^{13}$. Participants were also instructed to answer daily WhatsApp messages every morning for 19 days (August 10-28, 2019, 13 workdays and 6 free days) to record their actual sleep timing. Sleep logs (SL) allowed us to measure the average individual midsleep point of work (MSW) and free days (MSF) (Table 2).

Data are expressed as mean values \pm standard deviation throughout. As data did not comply with normality and/ or homoscedasticity, statistical comparisons were analyzed by non-parametric tests: the Wilcoxon signed-rank test for comparisons between work and free days in the same individuals, the Mann-Whitney $U$ test for comparisons across participants between shifts.

\section{RESULTS}

Twenty-nine dancers of the END-SODRE trained in the morning shift and 27 dancers trained in the night shift fulfilled the inclusion criteria to participate in this study (Table 1). Although earlier grades of the END-SODRE are scheduled in the night shift and last grades in the morning shift, the age of participants did not differ significantly across shifts ( $p=0.17$; Mann-Whitney $U$ test, Table 1 ).

The chronobiological characterization of the studied population was achieved using two largely validated questionnaires (MEQ and MCTQ), whose outputs were significantly correlated $(\mathrm{R}=-0.415, \mathrm{p}=0.0014$; Fig. 1A). Average chronotype corresponded to an MSFsc of 6:10 \pm 1:52, being significantly later in dancers attending the night shift $(6: 40 \pm 1: 52)$ than in morning-shift dancers $(5: 43 \pm 1: 47$ Table 1). Mean social jetlag was $2.03 \pm 1.71 \mathrm{~h}$ and correlated with MSFsc as expected $(\mathrm{R}=0.464, \mathrm{p}=0.0003$; Fig. 1B).
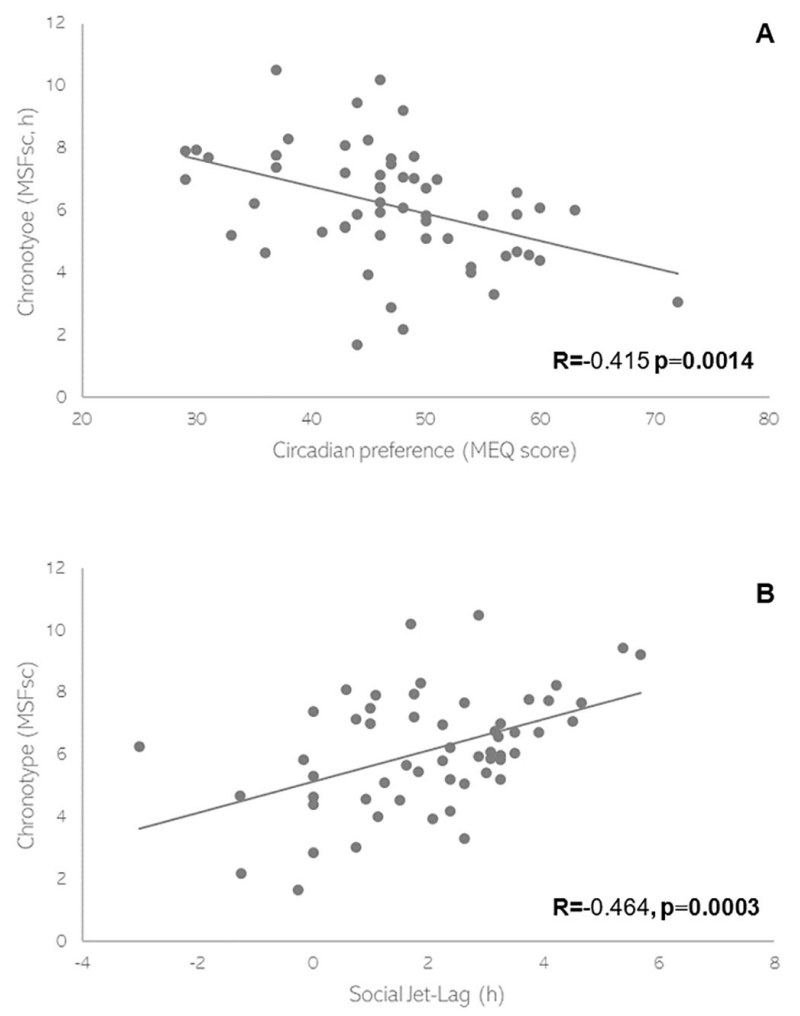

Figure 1. Linear regressions between midsleep point on free days corrected for sleep debt on workdays (MSFsc) and A) the score obtained from Morningness-Eveningness Questionnaire (MEQ); B) the social jet lag (SJL).

On the other hand, average circadian preferences corresponded to a MEQ score of $46.91 \pm 8.88$, with no significant differences between students attending the morning shift $(48.83 \pm 8.51)$ and the night shift $(44.85 \pm 8.96$; Table 1$)$.

Sleep timing was evaluated from the midsleep point calculated from data reported in MCTQ and SL, whose values were significantly correlated for both work $(\mathrm{R}=0.889, \mathrm{p}<$ 0.0001; Fig. 2A) and free days $(\mathrm{R}=0.534, \mathrm{p}<0.0001$; Fig. $2 \mathrm{~B})$. Both approaches consistently showed that sleep timing was significantly delayed in the free days respect to workdays in all the participants, being this delay longer in morning-shift dancers than in night-shift ones (Table 2). In addition, both MCTQ and SL data show that sleep is scheduled significantly later in nightshift dancers than in morning-shift ones in both work and free days (Table 2).

Table 2. Mid sleep point calculated using Munich Chronotype Questionnaire (MCTQ) and Sleep Logs for work (MSW) and free days (MSF), for dancers who attended morning-shift and night-shift.

\begin{tabular}{|c|c|c|c|c|c|c|}
\hline & & TTQ & & & Logs & \\
\hline & MSW & MSF & $\mathrm{p}^{1}$ & MSW & MSF & $\mathrm{p}^{1}$ \\
\hline \multirow[t]{2}{*}{ Morning-shift } & $3: 38 \pm 0: 34$ & $6: 26 \pm 1: 40$ & $<0.0001$ & $3: 34 \pm 0: 34$ & $6: 33 \pm 1: 26$ & $<0.0001$ \\
\hline & & & $\mathrm{n}=29$ & & & $\mathrm{n}=25$ \\
\hline \multirow[t]{3}{*}{ Night-shift } & $6: 08 \pm 1: 21$ & $7: 20 \pm 1: 29$ & 0.0027 & $5: 55 \pm 1: 05$ & $7: 07 \pm 1: 08$ & $<0.0001$ \\
\hline & & & $\mathrm{n}=27$ & & & $\mathrm{n}=25$ \\
\hline & $<0.0001$ & 0.0221 & & $<0.0001$ & 0.0407 & \\
\hline
\end{tabular}

\footnotetext{
Wilcoxon Matched-Pairs test
}

${ }^{2}$ Mann-Whitney U test 


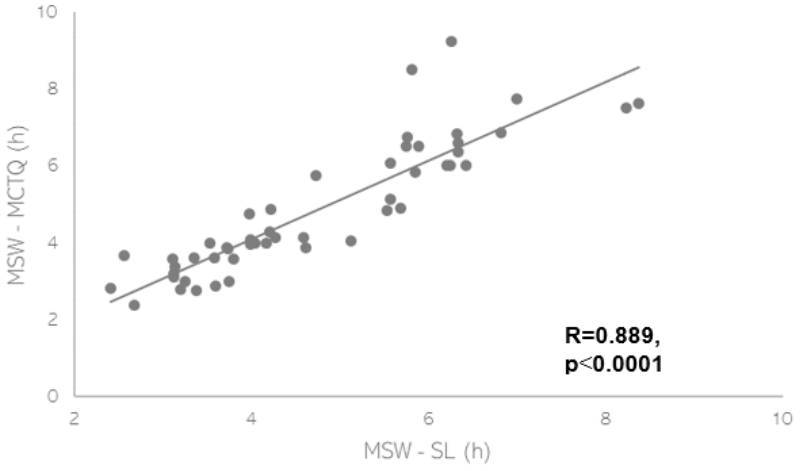

B

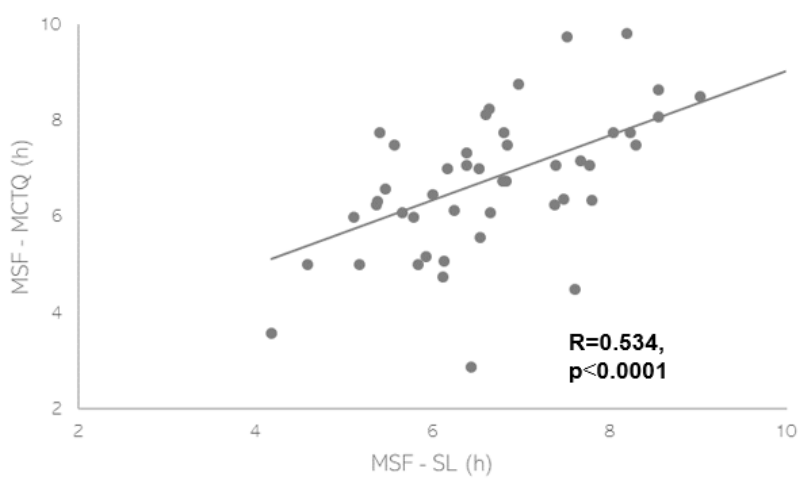

Figure 2. A) Workdays. Linear regression between midsleep point obtained from Munich Chronotype Questionnaire (MSW-MCTQ) and midsleep point obtained from Sleep logs (MSW-SL). B) Free days. Linear regression between midsleep point obtained from Munich Chronotype Questionnaire (MSF-MCTQ) and midsleep point obtained from Sleep logs (MSF-SL).

\section{DISCUSSION}

We present the chronobiological characterization of a group of young Uruguayan dancers being trained at the END-SODRE in two shifts, morning and night. Interestingly, although morning and night-shift dancers did not differ in their circadian preferences (measured by the MEQ score), individual chronotypes (estimated by MSFsc) were later in night shiftdancers respect to morning-shift ones.

The high quality of our data allows us to support our conclusions. First, as an internal validation of MCTQ, we found a significative correlation between MSFsc and social jet lag (Fig. 1A), indicating that later chronotypes are subjected to a significantly higher desynchronization as expected ${ }^{5}$. Secondly, we used two standard validated questionnaires (MCTQ and $\mathrm{MEQ}^{13,14}$ to do the chronobiological characterization of the study population, whose results were, as expected, significantly correlated (Fig. 1B $)^{18}$. Moreover, comparable data obtained from either the MCTQ questionnaire or the 19-days SL significantly correlated (Fig. 2), indicating the reliability of the self-reported information provided by the participants. Although age and gender differences in MSFsc have been previously reported ${ }^{3}$, we did not attempt to discriminate these effects given that all participants were over 18 years old, their age was constrained into a narrow range, and the study population was mostly composed by females.
As previously reported in Uruguayan youngsters, ${ }^{6,19}$, chronotypes measured by MSFsc were very late in average while MEQ scores were not suggestive of lateness. This discrepancy is not surprising as both questionnaires explore different aspects of circadian preferences and was also evinced in a similarage Uruguayan population ${ }^{20}$. Therefore, as the classification of circadian typologies depends on age, geographic, and cultural differences ${ }^{3}$, it is important to combine the use of different instruments to actually assess the chronobiological characterization of a given population.

Self-reported data either from MCTQ forms or from daily WhatsApp messages (SL), were very consistent in showing differences between the sleep patterns of morning and night shift dancers (Table 2; Fig. 2). Differences in dancers' sleep schedules between the morning and the night shift resemble those observed in Latin American adolescents attending different high school shifts ${ }^{6-9}$. In particular, this study is in accordance with these previous reports by showing that chronotypes (measured by MCTQ) are affected by the training shift, being later in the night shift than in the morning one; and that sleep is more advanced during workdays in morning-shift dancers compared to night-shift ones. Interestingly, to our knowledge, no previous studies have taken advantage of training in shifts to explore its chronobiological impact in young adults as most studies of this kind have been focused on high school adolescents.

Social demands impose a chronic misalignment between the inner and social clocks, particularly in adolescents and young adults, resulting in sleep deficiency during workdays and sleep compensation during weekends ${ }^{5,14}$. Although sleep duration was not analyzed in this study, it is evident that both morning and night-shift dancers followed the expected changes between work and free days, delaying the occurrence of sleep during weekends.

In conclusion, dancers being trained in morning and night shifts offer the opportunity to test the impact of these contrasting shifts on circadian preferences and sleep patterns. In this first study of this advantageous population, we confirm that sleep schedules show the expected differences between shifts and between work and free days. More interestingly, the indicator of chronotype that relies on self-reported sleep patterns is extremely late and delayed in night-shift dancers with respect to morning-shift ones; while the indicator of circadian preference does not report differences across shifts.

\section{ACKNOWLEDGMENTS}

We wish to thank very specially the dancers of the END-SODRE who kindly volunteered as participants of this study. We are specially thankful to the Director of the School of Performing Arts-SODRE, Martín Inthamoussou, and to the Coordinators of the END-SODRE, Andrea Salazar and Emiliano D'Agostino for their support and logistic help. We also thank Micaela De Mori, Agustín Laguardia, Betania Martínez, and Luna Perla for their help in data collection and Adriana Migliaro for her suggestion and comments that help us to improve the manuscript. 


\section{REFERENCES}

1. Adan A, Archer SN, Hidalgo MP, Di Milia L, Natale V, Randler C. Circadian typology: A comprehensive review. Chronobiol Int. 2012;29(9):1153-75.

2. Vink JM, Groot AS, Kerkhof GA, Boomsma DI. Genetic analysis of morningness and eveningness. Chronobiol Int. 2001;18(5):809-22.

3. Roenneberg T, Kuehnle T, Pramstaller PP, Ricken J, Havel M, Guth A, et al. A marker for the end of adolescence. Curr Biol. 2004:14(24):1038-9.

4. Roenneberg T, Kantermann T, Juda M, Céline V, Allebrandt K. Light and the Human Circadian Clock. In: Kramer A, Merrow M, editors. Circadian Clocks, Handbook of Experimental Pharmacology. Springer-Verlag Berlin Heidelberg; 2013. p. 311-31.

5. Wittmann M, Dinich J, Merrow M, Roenneberg T. Social jetlag: Misalignment of biological and social time. Chronobiol Int. 2006;23(1-2):497-509.

6. Estevan I, Silva A, Vetter C, Tassino B. Short sleep duration and extremely delayed chronotypes in Uruguayan youth: The role of school start times and social constraints. J Biol Rhythm. 2020;

7. Arrona-Palacios A, García A, Valdez P. Sleep-wake habits and circadian preference in Mexican secondary school. Sleep Med. 2015;16(10):1259-64.

8. Anacleto TS, Adamowicz T, Simões da Costa Pinto L, Louzada FM. School Schedules Affect Sleep Timing in Children and Contribute to Partial Sleep Deprivation. Mind, Brain, Educ. 2014;8(4):169-74.

9. Valdez P, Ramírez C, García A. Delaying and extending sleep during weekends: Sleep recovery or circadian effect? Chronobiol Int. 1996;13(3):191-8.

10. Hittle BM, Gillespie GL. Identifying Shift Worker Chronotype Implications for Health. Ind Health. 2018;56(6):512-23.

11. Gamble KL, Motsinger-Reif AA, Hida A, Borsetti HM, Servick S V., Ciarleglio CM, et al. Shift work in nurses: Contribution of phenotypes and genotypes to adaptation. PLoS One. 2011;6(4):1-12.
12. Estevan I, Silva A, Tassino B. School start times matter, eveningness does not. Chronobiol Int. 2018;35(12):1753-7.

13. Horne JA, Ostberg O. A self assessment questionnaire to determine Morningness Eveningness in human circadian rhythms. Int J Chronobiol. 1976;4(2):97-110.

14. Roenneberg T, Wirz-Justice A, Merrow M. Life between clocks: Daily temporal patterns of human chronotypes. J Biol Rhythms. 2003;18(1):80-90.

15. Genzel L, Ahrberg K, Roselli C, Niedermaier S, Steiger A, Dresler M, et al. Sleep timing is more important than sleep length or quality for medical school performance. Chronobiol Int. 2013;30(6):766-71.

16. Fischer FM, Nagai R, Teixeira LR. Explaining sleep duration in adolescents: The impact of socio-demographic and lifestyle factors and working status. Chronobiol Int. 2008;25(2-3):359-72.

17. Fietze I, Strauch J, Holzhausen M, Glos M, Theobald C, Lehnkering $\mathrm{H}$, et al. Sleep quality in professional ballet dancers. Chronobiol Int 2009;26(6):1249-62

18. Zavada A, Gordijn MCM, Beersma DGM, Daan S, Roenneberg T. Comparison of the Munich Chronotype Questionnaire with the Horne-Östberg's morningness-eveningness score. Chronobiol Int. 2005;22(2):267-78.

19. Tassino B, Horta S, Santana N, Levandovski R, Silva A. Extreme late chronotypes and social jetlag challenged by antarctic conditions in a population of university students from Uruguay. Sleep Sci. 2016;9(1):20-8

20. Silva A, Simón D, Pannunzio B, Casaravilla C, Díaz Á, Tassino B. Chronotype-Dependent Changes in Sleep Habits Associated with Dim Light Melatonin Onset in the Antarctic Summer. Clocks \& Sleep. 2019;1(3):352-66 


\section{Characterization of the circadian expression profile of clock genes in Aag2 cell line infected and uninfected by Dengue 2 virus (DENV2)}

Andresa Borges de Araujo Fonseca

Simone Morais da Costa ${ }^{2}$

Marcio Mantuano-Barradas ${ }^{3}$

Ada Maria de Barcelos Alves ${ }^{2}$

Rafaela Vieira Bruno ${ }^{1,4^{*}}$

\footnotetext{
${ }^{1}$ Laboratório de Biologia Molecular de Insetos, IOC, Fundação Oswaldo Cruz, Brazil.

${ }^{2}$ Laboratório de Biotecnologia e Fisiologia de Infecções Virais, IOC, Fundação Oswaldo Cruz, Brazil.

${ }^{3}$ Laboratório de Bioquímica de

Tripanosomatídeos, IOC, Fundação Oswaldo Cruz, Brazil.

${ }^{4}$ Instituto Nacional de Ciência e Tecnologia em Entomologia Molecular-CNPq/Brazil.
}

\begin{abstract}
Aedes aegypti is a mosquito vector of pathogens that cause important diseases. However, we don't know much about the molecular control of circadian rhythms in these insects. To understand how clock genes interact, we sought to establish the cell culture model for a detailed circadian study. The embryonic cell line Aag2 expresses the major clock genes. Thus, we characterized the circadian expression profile of clock genes in the Aag2 cells by qPCR in uninfected cells in LD12:12 and DD and in infected cells with DENV2 in LD12:12. Among the analyzed genes, only per showed a cyclic expression profile in these cells in both photoperiods. In cells infected with DENV2, we observed that no gene had a cyclic expression profile, although the expression pattern in infected cells is different from control ones, suggesting that for a gene cycling profile to occur, cell synchronization may be necessary.
\end{abstract}

Keywords: Aag2 Cells; Aedes Aegypti; Dengue Virus; Circadian Clock

\footnotetext{
Corresponding author:

Rafaela Vieira Bruno

E-mail: rafaelav@ioc.fiocruz.br

rafaelabruno@gmail.com
}

DOI: $10.5935 / 1984-0063.20200011$ 


\section{INTRODUCTION}

Aedes aegypti mosquitoes (Diptera: Culicidae) are vectors of several pathogens that cause important diseases such as Dengue Fever ${ }^{1}$. Aspects of mosquito behavior play a fundamental role in the dynamics of the diseases transmitted by them ${ }^{2}$. Despite its medical importance, we don't know much about the molecular control of circadian rhythms of these vectors. These rhythms, that have a period close to 24 hours under constant conditions (for example, in the absence of light/dark cycles), are controlled by an internal biological clock, that is under genetic control ${ }^{3,4}$. In insects, the molecular clock is well elucidated in model species Drosophila melanogaster and it is controlled by several genes interconnected in three negative feedback loops ${ }^{5}$.

On the other hand, Gentile et al. described the mRNA expression profile of the seven major genes of the first and second regulatory loops in Ae. aegypti. In general, an expression profile similar to that seen in Drosophila can be observed: period (per) and timeless (tim) genes have their peak expression in scotophase, while the peaks of Clock (Clk) and cycle (cyc) are in antiphase. The latter, in turn, presents a cyclic expression pattern, unlike Drosophila ${ }^{6}$. In addition, in mosquitoes of the Aedes genus, there are two forms of the cryptocrome gene: one similar to Drosophila's photoreceptor (cry1) and other (cry2), which is orthologous to the transcriptional repressor found in vertebrates ${ }^{6,7}$. The $c r y 1$ gene expression is constitutive, but cry2 expression has a bimodal pattern with two peaks of gene expression ${ }^{6}$. However, the importance of these two peaks in regulating the circadian clock is unknown.

Although the expression profile of these genes is already known, it's not clear how they interact with each other to regulate the circadian clock and other aspects of the biology of this important vector. Thus, the use of cell lines has been an alternative to the study of the circadian clock, especially in non-model organisms, as already seen for monarch butterflies ${ }^{8}$.

Aag2 cells ${ }^{9}$ are an embryonic lineage from Ae. aegypti and express the major clock genes, besides being immunocompetent cells, which makes these cells a good model for studying arbovirus infection, for example ${ }^{10}$. Therefore, this study aimed to establish the expression profile of the main clock genes in this cell line. We also infected these cells with DENV2, in order to obtain initial information on how the interaction between the clock and dengue virus infection could occur in this cell culture system, since it has been seen that female $A$ e. aegypti mosquitoes infected with DENV2 increase their locomotor activity pattern, which could suggest a relation between virus and clock genes ${ }^{11}$.

\section{MATERIAL AND METHODS}

\section{Cell culture}

Aedes aegypti Aag2 cell line was kindly provided by Dr. Marcos Sorgine from Universidade Federal do Rio de Janeiro (UFRJ) and were cultured in Schneider's Drosophila medium (Life Technologies) supplemented with 10\% fetal bovine serum (Life Technologies). The cells were maintained at $28{ }^{\circ} \mathrm{C}$ in $25 \mathrm{~cm}^{2}$ tissue culture flasks and passaged at 1:5 dilution every 3-4 days.

\section{Collection points of infected and non-infected cells}

For collection points, the cells were plated in $87 \mathrm{~mm}$ Petri dishes in Schneider's Drosophila medium with 10\% fetal bovine serum at appropriate dilution for each experiment. To establish the clock gene expression profile over 24 hours, a 1:3 dilution (for assays without infection) or a concentration of $24 \times 10^{5}$ cells $/ 10 \mathrm{~mL}$ (infection assays) was used. The cells were kept in a B.O.D incubator (Forlab - Eletrolab) at $28{ }^{\circ} \mathrm{C}$ without $\mathrm{CO}_{2}$ at LD12:12 (12 hours of light and 12 hours of dark) or DD (constant darkness). After 3 or 4 days at the desired photoperiod, the cells were collected every 4 hours until complete 24 hours and material was stored at $-80^{\circ} \mathrm{C}$ until RNA extraction.

\section{RNA extraction, cDNA synthesis and Real Time PCR}

Total RNA from cells was extracted using TRIzol reagent (Invitrogen), following the protocol described in Gentile et $a l^{6}$. cDNA was synthetized using TaqMan Reverse Transcriptase kit (Thermo Fisher) with oligo-dT according to the manufacture's protocol. After synthesis, cDNA was diluted 10X with milli-Q water. Quantitative Real Time PCRs (qPCRs) were performed in StepOne ${ }^{\mathrm{TM}}$ Real-Time PCR System (Applied Biosystems), using Power SYBR Green PCR Master Mix (Applied Biosystems), $4.0 \mu \mathrm{L}$ of cDNA and oligonucleotides at a concentration of $0.5 \mu \mathrm{M}$ in a final reaction volume of $15 \mu \mathrm{L}$. For the analyzed genes, we used the same pairs of oligonucleotides described in Gentile et al ${ }^{6}$, except for Clk (F: 5'- TCCTCCGGACTCGTCAACCGG-3'; R: 5'- TGCGCTGGAGTTAGCACGACG-3'). The reactions were performed in three technical replicates for each sample and independently for the analyzed genes, and data obtained from amplifications were analyzed using $\Delta \Delta \mathrm{Ct}$ method. RP49 gene was used as endogenous control of the reactions ${ }^{12}$.

\section{DENV2 infection in Aag2 cells}

A concentration of $24 \times 10^{5}$ cells was plated in Petri dishes in Schneider's Drosophila medium with 10\% fetal bovine serum 24 hours before infection. Given this time, cells were washed twice with phosphate-buffered saline (PBS) 1X and a serum free medium was replaced in all Petri dishes. The cells were incubated with $6,5 \times 10^{10} \mathrm{TCID}_{50}$ (tissue culture infectious dose $_{50}$ ) of DENV2, strain BR/RJ66985/2000 (GenBank register number: \#HQ012518) in $10 \mathrm{~mL}$, for an hour at $28^{\circ} \mathrm{C}$. The control group was incubated only with serum free medium. After that, the medium was changed again by culture medium with $10 \%$ fetal bovine serum and cells were placed in incubator at LD12:12 for 4 days until collection points.

\section{Statistical analysis}

Data from qPCRs were statistically analyzed by ANOVA followed by Tukey's test, using GraphPad Prism statistical software package (Prism 5.0; GraphPad Software, Inc., San Diego, CA). Asterisks indicate significant differences $\left({ }^{*} \mathrm{p}<0.05\right.$; $\left.{ }^{* *} \mathrm{p}<0.01 ; * * * \mathrm{p}<0.001\right)$. 


\section{RESULTS}

Because there were no available data about clock genes expression in Aag2 cell line in literature, we firstly performed Reverse Transcriptase PCR (RT-PCR) using cDNA from these cells, collected in two points throughout the day: ZT5 and ZT17. These points were chosen because, according to Gentile et at, these are the two moments that most of the clock genes present their peak expression in Ae. aegypti mosquitoes. The results showed that, the main genes described in Ae. aegypti: per, tim, Clk, cyc, cry1, cry2, vri and Pdp1, are expressed in Aag2 cells (data not shown).

Thus, we investigated whether the expression of these genes had a circadian profile. We performed qPCRs assays in two photoperiods: LD12:12 and DD. The experiments were carried out in four biological replicates for both analyzed photoperiods.

And finally, after establishing the expression profile of clock genes in LD (in ZTs 1, 5, 9, 13, 17 and 21) and DD (Cts 1, $5,9,13,17$ and 21), we investigated if DENV2 infection could modulate the expression of clock genes. The experiments were performed in three biological replicates in the photoperiod of 12 hours of light and 12 hours of dark.

\section{Clock gene expression profile in Aag2 cells maintained} at LD12:12 and DD.

In LD12:12, of the eight genes analyzed, only per gene is cyclically expressed, with a peak expression in ZT1 $(p=0.0066)$ (Figure 1). The other genes analyzed didn't show a cyclic expression profile in these cells. In other words, they are arrhythmic, although they appear to be widely expressed in Aag2 cells (Figure 2).

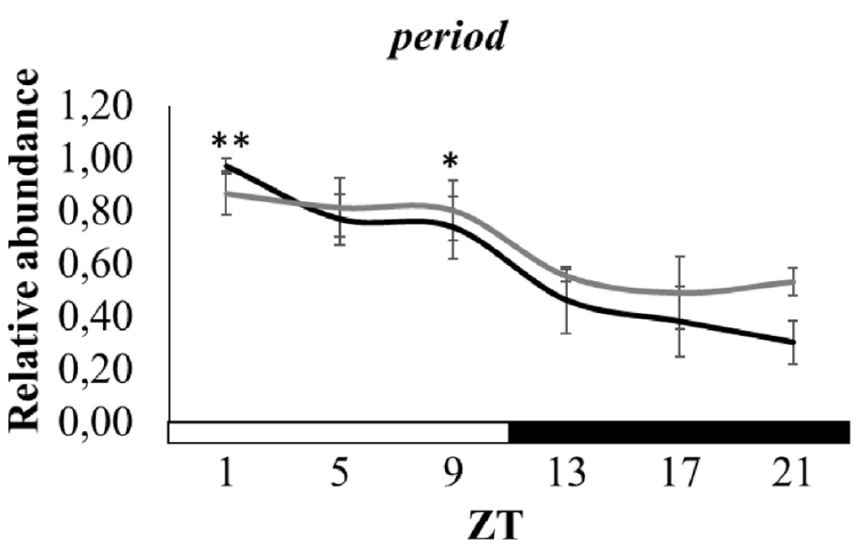

Figure 1. Circadian expression profile of clock genes by qPCR. The graph shows the expression of period gene in LD12:12 (black line) and DD (grey line). The y-axis indicates the relative mRNA abundance and the x-axis the time points (ZT). The graph was obtained from the average of four independent experiments. Asterisks represent statistically significant differences $\left({ }^{*} \mathrm{p}<0.01\right.$ in $\mathrm{LD} ;{ }^{*} \mathrm{p}<0.05$ in DD).

The next step was to investigate the circadian expression profile in the absence of environmental synchronizer (cells kept in constant darkness). In DD, per gene again showed a cyclic expression profile, however, without a defined expression peak, maintaining high expression levels between ZTs1 to $9(p=0.03)$ (Figure 1). The other genes remained arrhythmic in these cells (Figure 3).

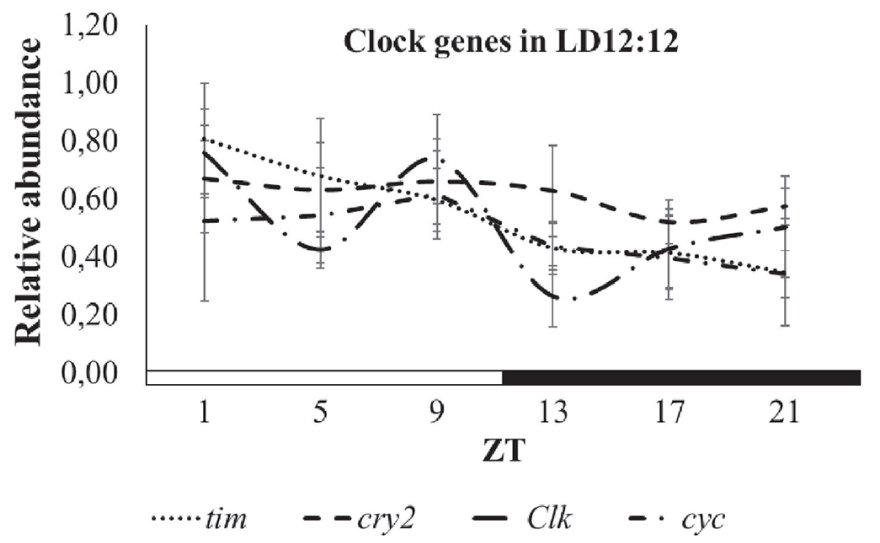

Figure 2. Circadian expression profile of clock genes by qPCR. The graph shows the expression of tim, cry2, Clk and $c y c$ genes in LD12:12. The $y$-axis indicates the relative mRNA abundance and the $\mathrm{x}$-axis the time points (ZT). The graph was obtained from the average of four independent experiments. Each gene is represented by a different line format as shown in the figure. The other analyzed genes (cry1, vrille and $P d p 1)$ are not represented in this figure. No significant difference was observed in any of the analyzed genes.

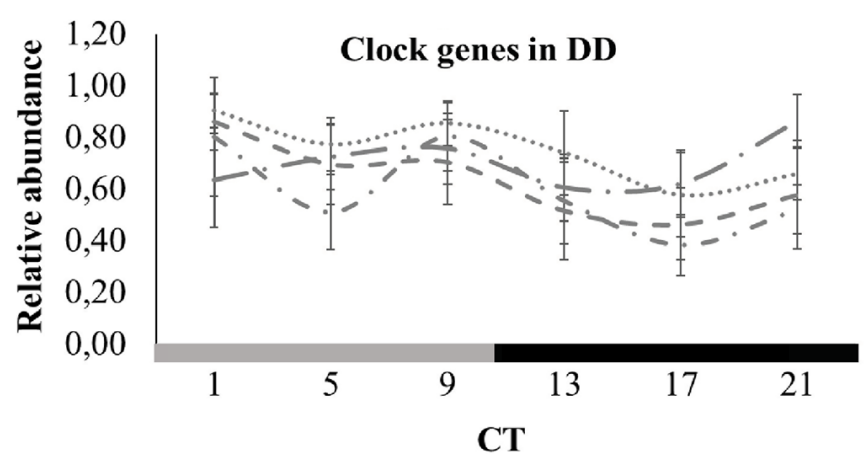

Figure 3. Circadian expression profile of clock genes by qPCR. The graph shows the expression of tim, cry2, Clk and $c y c$ genes in DD. The y-axis indicates the relative mRNA abundance and the $\mathrm{x}$-axis the time points (CT). The graph was obtained from the average of four independent experiments. Each gene is represented by a different line format as shown in the figure. The other analyzed genes $($ rry 1 , vrille and $P d p 1)$ are not represented in this figure. No significant difference was observed in any of the analyzed genes.

\section{Clock gene expression profile in DENV2-infected Aag2 cells in LD12:12}

Of the analyzed genes, none showed a cyclic expression profile in cells infected with DENV2. Moreover, in the uninfected control cells of the experiment, the clock genes didn't show a cyclic profile either. However, although arrhythmic, period showed a different expression profile between infected and uninfected cells (Figure 4).

As infection control, the percentage of cells infected by DENV2 was evaluated by flow cytometry. The overall mean infection was approximately $29 \%$ of the cells in the Petri dishes (data not shown). The results are different from those observed previously in LD12:12 because, in this second set of experiments, the cells were incubated for $1 \mathrm{~h}$ with serum-free medium, as it was part of the infection protocol. This most likely resulted in this loss of period gene cycling. 
period expression in infected cells

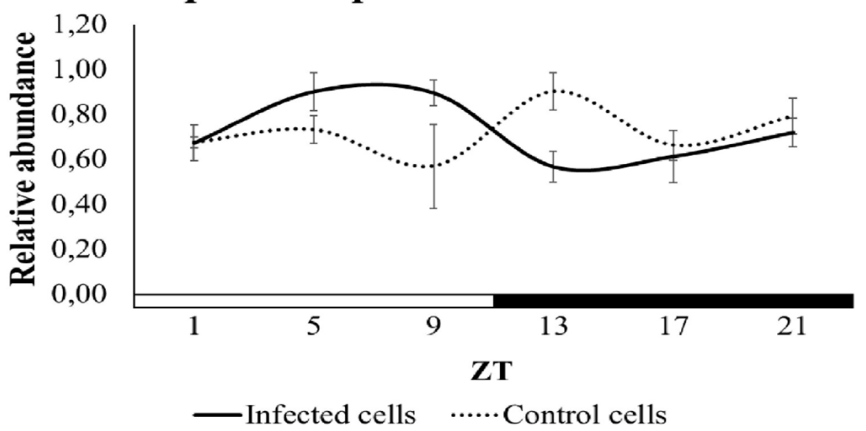

Figure 4. Circadian expression profile of period gene by qPCR. The graph shows per expression in cells infected with DENV2 in LD12:12. The y-axis indicates the relative mRNA abundance and the $\mathrm{x}$-axis the time points (ZT). The continuous line represents the gene expression in infected cells and the dotted line represents the gene expression in control cells. The graph was obtained from the average of three independent experiments. No significant difference was observed in any of the analyzed genes.

\section{DISCUSSION}

The expression profile of clock genes in heads of virgin females of Ae. aegypti was described by Gentile $e t a^{6}{ }^{6}$, and differs from the $D$. melanogaster model in some aspects, such as the cyclic expression of cycgene and presence of a second form of cryptocbrome gene (cry2). However, the absence of known mutant alleles for these genes makes research on the molecular clock of mosquitoes a descriptive rather than functional task. Therefore, an alternative found by our group to perform functional studies on the circadian clock in Ae. aegypti was to use the Aag2 cell line, derived from the species itself. We began characterizing the clock gene expression profile by qPCR, synchronizing to light/dark cycles.

When Aag2 cells are submitted a light/dark cycles, only one gene is cyclically expressed: period, with a peak expression during the light phase. The other clock genes, which in mosquito female heads are rhythmic ${ }^{6}$, in this lineage are arrhythmic. Interestingly, whereas in Aag2 cells the per gene expression peak is in ZT1, in mosquitoes, this expression peak occurs only in ZT17, during the dark phase of a light/dark cycle.

The Aag2 lineage is derived from embryonic cells, so it's possible that the expression profile of clock genes is different from that found in adults. This arrhythmic pattern of clock genes expression is not surprising in insect's cell lines. D. melanogaster S2 embryonic cells also have an arrhythmic expression profile, and don't even express some of the canonical circadian clock genes, such as per and $c y c^{13,14}$.

Studies with $D$. melanogaster embryos show that during embryogenesis, from embryonic stage 12 (ES12), PER protein is already expressed in the ventral nerve chord and in ES15, it's expressed in brain cells. However, at these stages no CLOCK protein is expressed, indicating that transcription of per gene at this time of embryogenesis isn't being activated by CLK-CYC transcription factors, as in adult $\mathrm{fly}^{15}$. These data suggest that per may not be regulated by canonical activators (CLK-CYC) of the circadian clock in D. melanogaster embryos.
This could explain the results seen in Aag2 cells, suggesting that per gene may be regulated independently of Clk and $c y c$.

In $\mathrm{DD}$, per gene also showed a cyclic expression profile during the subjective day, and the other genes presented an arrhythmic expression profile as we observed in LD12:12. These data are not surprising since it's known that constant conditions lead to decreased rhythm amplitude or even arrhythmicity. For example, DpN1 embryonic cells from the monarch butterfly Danaus plexippus also express various circadian clock genes, showing rhythmicity in LD12:12, but all lose the cycling profile in DD as seen in Aag2 cells8.

However, the fact that the circadian clock is not functional doesn't mean that the cell isn't under oscillatory control. Rey et al. showed that $D$. melanogaster $\mathrm{S} 2$ cells that don't express several canonical circadian clock genes have proteins, in particular metabolic enzymes, which are expressed rhythmically over a 24hour period ${ }^{14}$. This strongly suggests that there is a circadian clock acting. The hypothesis is that the cyclic expression of these enzymes could be being regulated at post-translational level, which could also be happening with Aag2 cells.

Although we didn't see a cyclic expression profile of most clock genes in these cells, we infected them with DENV2, because there are data in the literature showing that pathogen infection may modulate the circadian clock, as seen in D. melanogaster, where, mutant flies to clock genes are more susceptible to pathogenic bacterial infections when compared to wild flies, for example ${ }^{16}$.

However, no gene had a cyclic expression profile (cells infected with DENV2 and control cells), not even the per gene, which presented rhythmicity in both analyzed photoperiods. One hypothesis for the loss of per gene rhythmicity is that during virus adsorption, cells are subjected to $1 \mathrm{~h}$ serum free medium (control cells were incubated only by serum free medium, that is, without virus). This absence of serum could interfere in per gene cycling, as according to standardized protocols, serum shock is an efficient way to synchronize the cell cycle and consequently promote the cycling of clock genes in mammalian cells, for example ${ }^{17}$. In this work, we chose not to synchronize the cell cycle, since studies with insect cells didn't use this approach to analyze the clock gene expression profile ${ }^{8,12}$. However, given the results obtained, synchronization may be a methodology to be used, so that the clock genes present a cyclic expression profile in these cells.

Although it is not possible to affirm that Aag2 cells have a functional circadian clock, these cells express all canonical clock genes, indicating that further studies are still needed to understand if these cells can be a model for initial and complementary studies on circadian clock in Ae. aegypti.

\section{ACKNOWLEDGEMENTS}

We thank Paolla Pinto for laboratorial assistance and Robson Costa for technical support. 


\section{REFERENCES}

1. Ruckert C, Weger - Lucarelli J, Garcia - Luna SM, Young MC, Byas AD, Murrieta RA, et al. Impact of simultaneous exposure to arboviruses on infection and transmission by Aedes aegypti mosquitoes. Nat. Commun. 2017; 8, p.15412.

2. Klowden MJ, Zwiebel L. Vector Olfaction and Behavior. In Biology of Disease Vectors 2nd Edition. ed. Marquardt W.C. et al. Elsevier Academic Press. Amsterdam. 2004.

3. Saunders DS. Insect Clocks.3rd edition Elsevier Science. Amsterdam. 2002.

4. Hall J.C. Genetics and molecular biology of rhythms in Drosophila and other insects. Adv. Genet. 2003; 48:1-280.

5. Hardin P.E. Molecular genetic analysis of circadian timekeeping in Drosophila. Adv. Genet. 2011; 74, 141-73.

6. Gentile C, Rivas GBS, Meireles-Filho ACA, Lima JBP, Peixoto AA. Circadian expression of clock genes in two mosquito disease vectors: cry2 is different. J Biol Rhythms. 2009; 24: 444-51.

7. Yuan Q, Metterville D, Briscoe AD, Reppert SM. Insect cryptochromes: gene duplication and loss define diverse ways to construct insect circadian clocks. Molec Biol Evol. 2007; 24 (4), 948-55.

8. Zhu H, Sauman I, Yuan Q, Casselman A, Emery-Le M, Emery P, et al. Cryptochromes define a novel circadian clock mechanism in monarch butterflies that may underlie sun compass navigation. Plos Biology. 2008; $6(1), 138-155$
9. Peleg J. Growth of arboviruses in monolayers from subcultured mosquito embryo cells. Virology. 1968; 35 (4):617-9

10. Barletta ABF, Silva MCN, Sorgine MHF. Validation of Aedes aegypti Aag2 cells as a model for insect immune studies. Parasit. Vectors. 2012; 5(1): 1-9.

11. Lima-Camara TN, Bruno RV, Luz PM, Castro MG, Lourenço-de-Oliveira $\mathrm{R}$, Sorgine, $\mathrm{MHF}$, et al. Dengue infection increases the locomotor activity of Aedes aegypti females. PLoS One. 2011; 8, e17690, 2011.

12. Gentile C, Lima JBP, Peixoto AA. Isolation of a fragment homologous to the $r p 49$ constitutive gene of Drosophila in the Neotropical malaria vector Anopheles aquasalis (Diptera: Culicidae). Mem. Inst. Oswaldo Cruz. 2005; Vol.1000 (6): 545-7.

13. Nawathean P, Menet JS, Rosbash M. Assaying the Drosophila negative feedback loop with RNA interference in S2 cells. Methods Enzymol. 2007; 393. 610-22.

14. Rey G, Milev NB, Valekunja UK, Ch R, Ray S, Silva Dos Santos M, et al. Metabolic oscillations on the circadian time scale in Drosophila cells lacking clock genes. Mol. Syst. Biol. 2018; 14(8):1-11

15. Houl JH, Ng F, Taylor P, Hardin P. CLOCK expression identifies developing circadian oscillator neurons in the brains of Drosophila embryos. BMC Neurosci. 2008; 9: 1-13.

16. Lee J, Edery I. Circadian regulation in the ability of Drosophila to combat pathogenic infections. Curr Biol. 2008; 18(3): 195-9.

17. Balsalobre A, Damiola F, Schibler U. A Serum Shock Induces Circadian Gene Expression in Mammalian Tissue Culture Cells. Cell. 1998; 93:929_937. 


\section{Social modulation on daily variability in electric behavior}

Valentina Gascue

Ana Silva

Adriana Migliaro *

Laboratorio de Neurociencias, Facultad de Ciencias, Universidad de la República, Montevideo, Uruguay.

\begin{abstract}
Daily rhythms of behavior often result from the expression of a circadian rhythm modulated and synchronized by abiotic and biotic cues from the environment. Circadian rhythms allow living organisms to anticipate changes and allocate energy in order to cope with predicted events as well as to time behavioral displays in social contexts. Understanding the complexity of behavioral rhythmicity requires a more comprehensive analysis that takes into consideration the intricacy of natural environmental constrains, in the wide range of modulating factors operating in nature. Gymnotus omarorum is a pulse type gymnotiform widely distributed in Uruguay, which constantly displays an electric behavior, with a circadian rhythm of nocturnal increases in its rate of emission, that serves communicative and perceptual purposes. Given its fundamental role, the electric behavior needs to be a reliable signal especially in social contexts. In this report, we aim at analyzing the daily changes in the variability of the electric behavior as well as the modulatory effect of the social context on this variability.
\end{abstract}

Keywords: Circadian Rhythms; Electric Behavior; Electric Fish; Social Synchronization 


\section{INTRODUCTION}

Daily rhythms of behavior often result from the expression of a circadian rhythm modulated and synchronized by abiotic and biotic cues from the environment. Light and temperature, but also predatory activity and conspecific interactions, are important synchronizers of endogenous rhythms ${ }^{1,2}$. Circadian rhythms allow living organisms to anticipate changes and allocate energy in order to cope with predicted events as well as to time behavioral displays in social contexts. Classical studies in precisely controlled laboratory conditions have led to a simplified, dichotomic view, of either nocturnal or diurnal patterns. This is especially so when reducing behavior to a single variable, the most commonly used being locomotor activity. Understanding the complexity of behavioral rhythmicity requires a more comprehensive analysis that takes in into consideration the intricacy of natural environmental constrains in the wide range of modulating factors operating in nature, as well as the different expressions of behavior displayed by individuals of the species ${ }^{3}$.

Nocturnal fishes of the South American order Gymnotiformes are characterized by the emission of speciesspecific weak electric discharges that serve electrosensory and electrocommunication purposes ${ }^{4,5}$. These typical electric behavior consists of pulse discharges (the "electric organ discharge", EOD) continuously emitted by a specialized electric organ. A medullary pacemaker nucleus commands these emissions, setting the basal rate of the EOD (EOD-BR $)^{6}$ while modulated by central connections from pre-pacemaker structures. The EOD is a behavioral display that encodes information (in the waveform and frequency domains) about an individual's species identity, sex, and physiological state 7 . In addition, the EOD is the physical carrier of perceptually relevant sensory information ${ }^{8}$. Arousal state in weakly electric fish implies an increase in EOD basal rate hence increasing the availability of sensory information per unit of time. Exploratory movements, novelty detection, escape responses and even volition are associated with increases in EOD-BR ${ }^{1}$. As arousal coincides with the active phase of the day, the EOD-BR in these nocturnal animals increases during the night ${ }^{1,9-12}$.

Gymnotus omarorum is a pulse type gymnotiform widely distributed in Uruguay ${ }^{13}$. The nocturnal increase in EODBR is a circadian rhythm ${ }^{14}$, depends on melatonin ${ }^{15}$ and is expressed in the natural habitat even though the surrounding vegetation generates a constantly dark environment ${ }^{1}$. Moreover, the rhythmic nocturnal rise in EOD-BR is precisely timed to the daily maximum temperature of the water, rendering this cue the most likely environmental zitegeber. Social context is a fundamental circadian synchronizer in natural populations, however overlooked when analyzing the rhythmic expression of natural behaviors. The nocturnal increase in EOD-BR is indeed strongly influenced by the social context, which exerts a potent synchronization effect among animals in the same population ${ }^{1}$.

Given its fundamental role the EOD needs to be a reliable signal, yet modifiable to accommodate the aforementioned necessary changes. Previous reports for this species have shown that the EOD-BR has an intrinsic low variability when measured in isolated animals in a resting condition at laboratory settings.
Moreover, EOD-BR variability shows an exact correlation with the variability of the spontaneous discharge of the central pacemaker that triggers de $\mathrm{EOD}^{16}$. It is interesting to consider how is this system behaving in the natural habitat, in which individuals are challenged by a more complex and changing environment. A high variability in the rate of emission of the EOD likely increases its uncertainty as electrosensory signal, and hinders the performance of both, communicative and perceptual channels. In this report, we aim at analyzing the daily changes in the variability of the electric behavior and the modulatory effect of the social context on this variability.

\section{MATERIAL AND METHODS}

Adult G. omarorum ${ }^{13}$ ( $\left.\mathrm{n}=11\right)$ were used in natural settings including the recording of 6 individuals in natural conditions and 5 individuals isolated in shelters within the natural habitat (seminatural condition). All specimens were collected in Laguna del Sauce, Maldonado, Uruguay ( $34^{\circ} 48^{\prime} \mathrm{S}, 55^{\circ} 18^{\prime} \mathrm{W}$ ). Fish were located using a fish detector, consisting in an electronic audio amplifier connected to a pair of electrodes, as described elsewhere ${ }^{17}$ ).

The experiments were conducted during the nonbreeding season at the peri-equinox period, under a natural lightdark cycle of 12:12. Periodic light and temperature measures were taken each 30 minutes: a) inside the water under the natural vegetation and $\mathrm{b}$ ) outside the water (HOBO-MicroDAQ: UA002-08). Measurements range: Temperature: $-20^{\circ}$ to $70^{\circ} \mathrm{C}\left(-4^{\circ}\right.$ to $158^{\circ} \mathrm{F}$ ); Light: 0 to 320,000 lux (0 to 30,000 lumens/ft2).

All research procedures complied with ASAP/ABS Guidelines for the Use of Animals in

Research and were approved by the Institutional Ethical Committee (Comisión de Ética en el Uso de Animales, Instituto Clemente Estable, MEC, 008/11).

\section{EOD-BR recordings in social context}

EOD-BR was recorded during 72 hs from fish $(n=6)$ placed in individual plastic nets with electrodes, under the natural vegetation, in their natural habitat. 30 s recordings were made once an hour. Fish in these conditions are almost always detectable. As fish can move around while the electrodes in the plastic nets remain in a fixed position, the amplitude and waveform of their EOD usually changes. The natural social context is preserved as plastic meshes are electrically transparent, enabling the perception of conspecifics' electric signals. Conspecifics in this setting might be freely moving fish or other enclosed fish placed nearby.

\section{EOD-BR recordings in isolated animals}

Fish ( $\mathrm{n}=5)$ were placed in $50 \mathrm{~L}$ individual plastic tanks containing a shelter and equipped with a pair of electrodes attached to its sides. Given the size of the shelters, when recordings show stable EOD amplitudes, it can be reliably assumed that fish are still while sheltered and hence in a voluntary locomotor rest. EOD-BR was recorded for 30 seconds per hour during $24 \mathrm{~h}$, only if fish were sheltered and hence still. Fish in this condition are isolated from the influence of conspecifics. Light and temperature were monitored as described earlier. 


\section{Data processing and statistical analysis}

The EOD was recorded through electrodes placed in the water, digitalized using standard computer soundcards and recorded with a custom developed Matlab (The MathWorks, Inc.) program which detects the moment of EOD occurrence.

EOD-BR was calculated as the inverse of the inter EOD intervals in the recordings and expressed in terms of the median \pm MAD values. As fish differ in their individual EOD-BR an index (BRIn) was calculated to determine the increase between the EOD-BR measured an hour before sunset $\left(\mathrm{BR}_{-60}\right)$ and the values measured at sunset $\left(\mathrm{BR}_{\text {sunset }}\right)$, regardless absolute values for each fish. The global BRIn for the whole group was calculated as the median \pm MAD value of individual indexes.

BRIn $=($ BR-60-BRsunset $) / B R-60$

In order to normalize the effect of water temperature on EOD-BR, values were corrected to a constant $20^{\circ} \mathrm{C}$ temperature by using the $Q_{10}$ value of 1.5 as calculated for electric fish ${ }^{11} . Q_{10}$ is a unitless quantity calculated as the factor by which the rate increases when the temperature $(\mathrm{T})$ is raised by ten Celsius degrees.

$\mathrm{Q} 10=\mathrm{EOD}-\mathrm{BR} *(\mathrm{~T}) / \mathrm{EOD}-\mathrm{BR} *(\mathrm{~T}+10)$

Paired non-parametrical two-tailed Wilcoxon test was used for statistical analysis. Data are shown as median \pm MAD.

We studied the differences in variability between day and night for all fish (social and isolated). Qualitative and quantitative analysis were performed to each set of data by implementing an ad hoc routine in Matlab. We calculated variance, and coefficient of variation according to the following equations:

Variance

$$
\sigma^{2}=\frac{1}{N-1} \sum_{i=1}^{N}\left(x_{i}-\overline{X_{N}}\right)^{2}
$$

Coeficient of variation

$$
C V=\frac{\sigma}{\overline{X_{N}}}
$$

Poincaré diagrams were plotted as a means to obtain a qualitative analysis of dispersion. Each observed value of a set of data is plotted against the following, i.e. each EOD's frequency value $(\mathrm{BR})$ against the following $(\mathrm{BR}+1)$. These types of diagrams are intimately related to the system's variability and can be quantified. To mathematically characterize the information given by this diagram, an ellipse if fitted to the graph and the longitudinal and perpendicular axes (SD1 and SD2) of such ellipse are measured. The perpendicular (SD1) and longitudinal (SD2) radius of this ellipse represent long and short term variability of the system respectively. Poincaré diagrams were plotted separately for each individuals' day and night values of EOD-BR. Longitudinal and perpendicular axes were measured separately for each diagram giving a value for long term variability during night and day $\left(\mathrm{SD} 1_{\text {night }}, \mathrm{SD} 1_{\mathrm{dar}}\right)$ and for short term variability during night and day $\left(\mathrm{SD} 2_{\text {night }}, \mathrm{SD} 2_{\mathrm{day}}\right)$.

\section{RESULTS}

The EOD-BR of G. omarorurm consistently increases during the night in the natural habitat, with a mean EOD-BR $12 \%$ higher at sunset than $60 \mathrm{~min}$ before (EOD-BrIn=0,12 \pm $0,05)\left(\mathrm{FB}_{\text {sunset }}\right.$ vs FB-60; $\mathrm{n}=6$; Wilcoxon test, $\left.\mathrm{p}=0.02\right)$. Figure $1 \mathrm{a}$ shows the nocturnal increase EOD-BR for a single individual along the 72 hs recorded. This increase is paired with a decrease in variability as shown in the Poincare plot in $1 \mathrm{~b}$. Ellipses are fitted at the mean EOD-BR both for the night (29.3) and the day hours (27.1). Long and short term variability are measured by the longitudinal (SD1) and perpendicular (SD2) axes of the ellipses. SD1 values are is lower during the night (SD1 ${ }_{\text {night }}$ vs SD1 $1_{\text {day }}$, Wilcoxon $n=6, p=0.02$ ) and the same holds true for $\mathrm{SD} 2$ axes (SD2 $2_{\text {night }}$ vs SD2 $2_{\text {day }}$, Wilcoxon $\left.\mathrm{n}=6, \mathrm{p}=0,05\right)$ as shown in figure 1c. Night and day variance was calculated in order to quantify variability. Variance is also significantly lower at night across the population (Wilcoxon $n=6, p=0.02$ ) (figure 1d).

When individuals are recorded in the natural habitat inside plastic tanks social context is removed, not affecting the nocturnal increase in EOD-BR. Animals in this group show a mean EOD-BR 17.5\% higher at sunset than in the previous hour $(\mathrm{EOD}-\mathrm{BrIn}=0,175 \pm 0.09)\left(\mathrm{FB}_{\text {sunset }}\right.$ vs $\mathrm{FB}_{-60}$; $\mathrm{n}=5$; Wilcoxon test; $\mathrm{p}=0.04)$. Figure $2 \mathrm{a}$ shows the nocturnal increase EOD-BR for a single isolated individual along $24 \mathrm{hs}$. Poincare plots for isolated individuals show the aforementioned difference in EOD-BR, since the ellipse fitted to day values is centered at a lower (31.4) position that the ellipse fitted to the night values (34.1). Figure $1 \mathrm{~b}$ shows an example individual. However, an analysis of both axes of the ellipses shows no difference in variability in these two conditions (figure 1c). Variance calculated for all the individuals shows no difference between day and night (figure $2 \mathrm{~d}$ ).

The EOD-BR variability decreases during the night exclusively in animals in social context. In order to compare this set of data, which do not have the same mean, we calculated the coefficient of variation. Figure 3 shows the variation coefficient for each condition both during the night and the day. In social animals the coefficient of variation is significantly lower during the night than during the day (Wilcoxon, $\mathrm{n}=6, \mathrm{p}=0.02$ ). Moreover, variability during the night is significantly lower in social animals than in isolated animals (Mann-Whintney, $\mathrm{p}=0.04$ ).

\section{DISCUSSION}

Natural behavior relays on a precise timing that allows synchronization among conspecifics and with the natural cycles of the environment. Circadian clocks alone are not enough to sustain these adaptive rhythms and need to be successfully modulated by the environment and social context. In nature, the behavioral repertoire has a wider range $^{3}$ and social interactions are a fundamental source of behavioral diversity. Social context exerts a synchronization effect on the dynamics of the daily cycle of electric behavior ${ }^{1}$ 
A

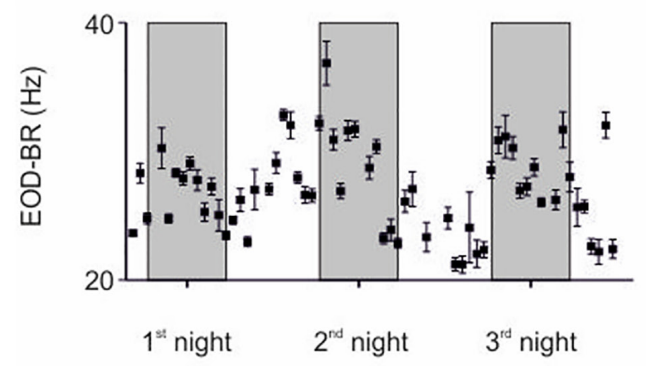

C

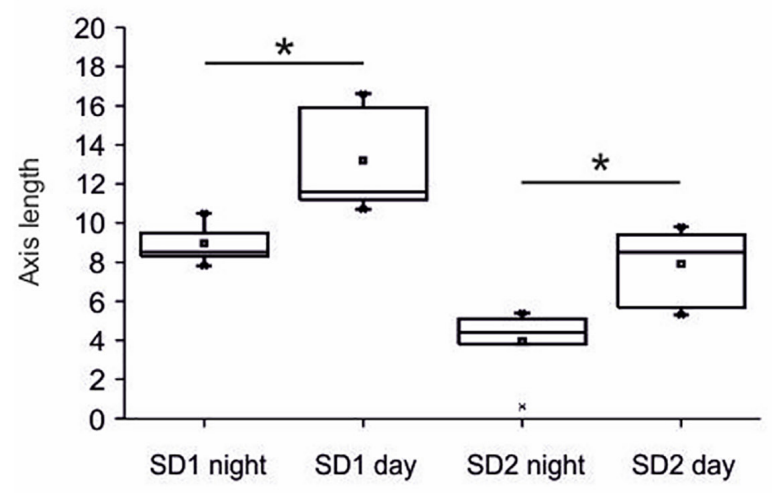

B

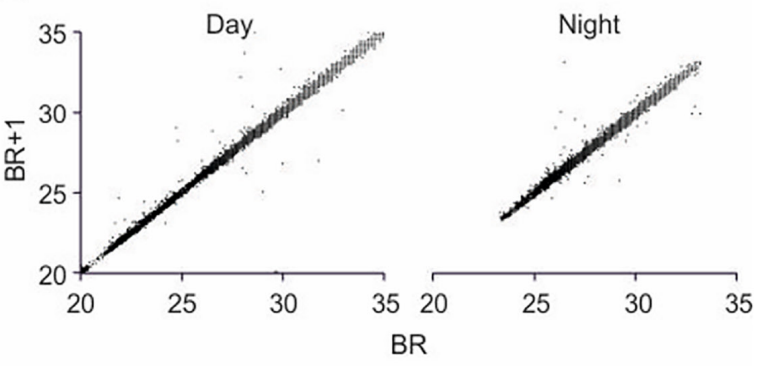

D

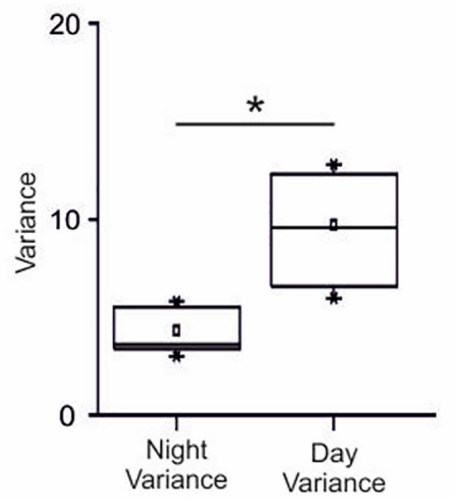

Figure 1. Daily analysis of the variability of EOD-BR in social fish. A) EOD-BR of a fish recorded in its natural habitat for 72 hs. Mean frequency values calculated for each hour swing daily with higher values at night (dark rectangles, sunset $7 \mathrm{pm}$, sunrise $7 \mathrm{am}$ ). B) Poincaré plots for the day and night EOD-BR values of a single fish, showing the higher dispersion of daily values. C) Quantitative analysis of Poincare plots based on the comparison of the longitudinal (SD1) and perpendicular (SD2) axes of an ellipse fitted around the points of the graphical representation shown in B. Variability in significantly lower during the night regardless the axis considered (SD1night vs SD1day, Wilcoxon $\mathrm{n}=6$, $\mathrm{p}=0.02$; SD2night vs SD2day, Wilcoxon $\mathrm{n}=6, \mathrm{p}=0,05)$. D) Variance, a quantitative measure of variability also shows the lower night time variability (Wilcoxon $\mathrm{n}=6$, $\mathrm{p}=0.02)$.

A

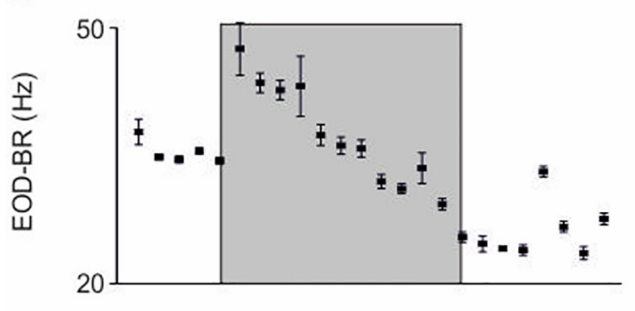

B

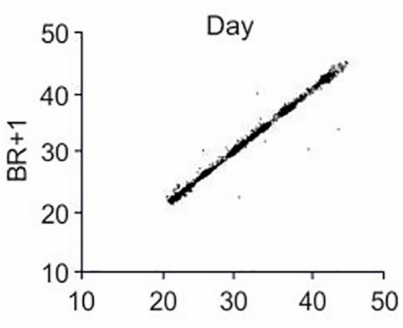

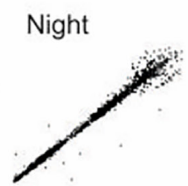

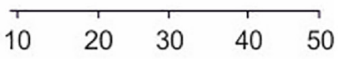

BR
C

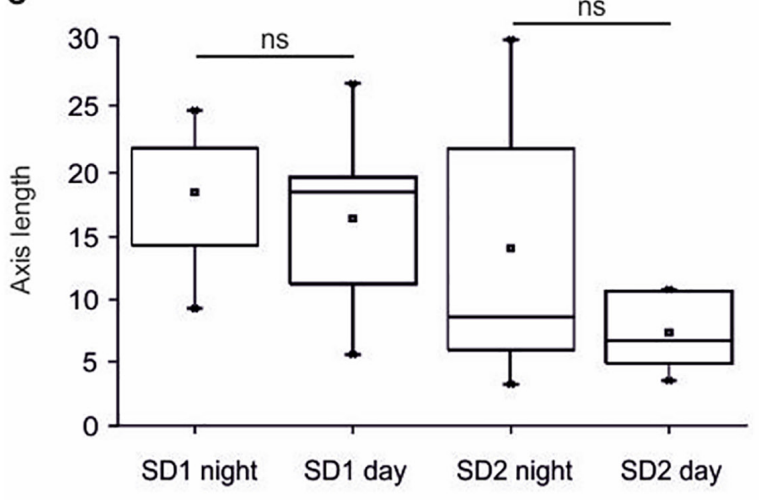

D

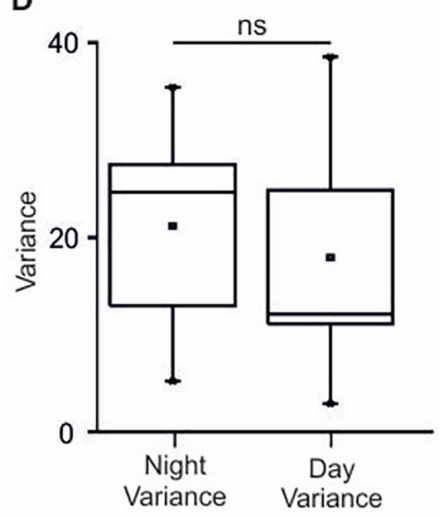

Figure 2. Daily analysis of the variability of EOD-BR in isolated fish. A) EOD-BR of a fish recorded in its natural habitat in isolation conditions for 24 hs. Mean frequency values calculated for each hour swing daily with higher values at night (dark rectangle, sunset 7pm, sunrise 7 am). B) Poincaré plots for the day and night EOD-BR values of a single fish, showing the relative dispersion of frequency values. C) Quantitative analysis of Poincaré plots based on the comparison of the longitudinal (SD1) and perpendicular (SD2) axes of an ellipse fitted around the points of the graphical representation shown in B. Variability is not significantly during the different phases of the day regardless the axis considered. D) Variance, a quantitative measure of variability also shows same variability during day and night. 


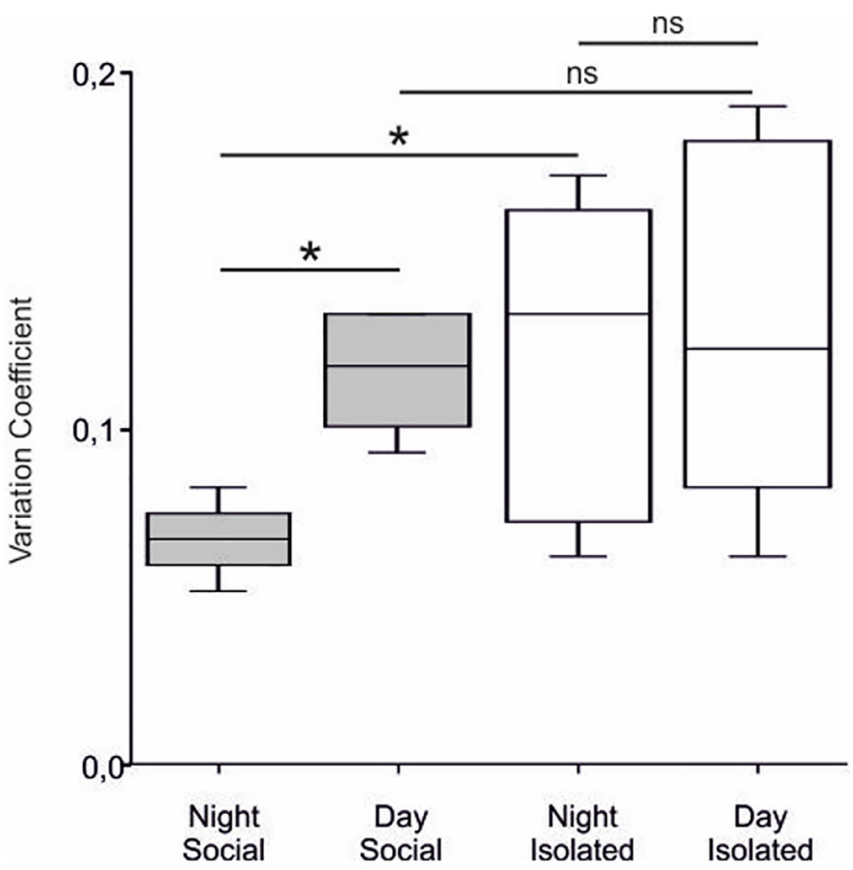

Figure 3. Coefficients of variation for night and day values of EOD-BR in social and isolated individuals. Social context lowers variability during the night (Night social vs Night isolated, Mann-Whintney, $\mathrm{p}=0.04$ ) and allows the emergence of a daily difference of this trait (Night social vs Day social, Wilcoxon, $n=6, p=0.02$ ).

We show in this work that synchronization is paired by a reduction in variability which is particularly evident at night, when social interactions and exploratory activity tend to be more frequent'. At night, animals inhabit an enriched environment where information needs to be conveyed in a reliable and efficient way.

The EOD-BR of G. omarorurm increases during the night in both social and isolated animals. This increase, which has also been reported for animals in laboratory conditions ${ }^{15}$, is sustained by a circadian rhythm of nocturnal increase ${ }^{14}$. The nocturnal increase in EOD-BR in laboratory settings is milder and shorter lasting than the one recorded in nature, evidencing the enhancing effect exerted by this enriched natural context. Moreover, social interactions, even the ones occurring in laboratory settings, have been shown to potentiate the nocturnal increase in electric behavior on the related species Brachybypopomus gauderio $0^{11}$.

A more detailed analysis of electric behavior reflects further effects of social context on its expression. When EOD-BR variability is assessed in a natural context there is a clear daily rhythm, with a significant nocturnal reduction in variability accompanying nocturnal increase in EOD-BR. However, when animals in the natural habitat are isolated from their conspecifics, time intervals between EODs became more variable regardless of daytime. This finding adds new evidence to the claim for the importance of taking into account different behavioral parameters for temporal analysis, as well as to establish the role of the multiple different factors that play a relevant timing role in the natural habitat.
The EOD is a complex signal that carries information serving communicative and perceptual goals. Since the variability of a signal correlates with its entropy ${ }^{18}$ or uncertainty, a greater regularity in nocturnal EOD-BR might be ensuring a more reliable scenario for electrocommunication and electroreception. Moreover, social context in other teleosts, as well as in mammals, has been shown to induce a reduction in variability and quantity of locomotor and exploratory activity ${ }^{19,20}$, suggesting a common effect of conspecific presence on motivation for behavioral displays. Unpublished data from our group shows the same effect of social context on locomotor activity, in the annual fish Austrolebias reicherti. Behavior, in order to provide an advantage for the species displaying it, needs to be adequately timed, a challenging task in a variable environment. However, the natural variability of environmental clues and social context yields a coordinating and timing effect on behavioral displays, which, when expressed in nature, tend to be more organized and less variable giving rise to a more adaptive repertoire.

\section{ACKNOWLEDGEMENTS.}

The authors wish to thank the organization of the XV Latin American Symposium on Chronobiology for including this work in the Symposium's special issue

\section{REFERENCES}

1. Migliaro, A., et al., Daily changes in the electric behavior of weakly electric fish naturally persist in constant darkness and are socially synchronized. Biol Open, 2018. 7(12).

2. Fuchikawa, T., et al., Potent social synchronization can override photic entrainment of circadian rhythms. Nature Communications, 2016. 7(1): p. 11662.

3. Hut, R.A., et al., In search of a temporal niche: environmental factors. Prog Brain Res, 2012. 199: p. 281-304.

4. Lissmann, H., On the function and evolution of electric organs in fish. Journal of Experimental Biology, 1958. 35(1): p. 156-191.

5. Stoddard, P.K., The evolutionary origins of electric signal complexity. J Physiol Paris, 2002. 96(5-6): p. 485-91.

6. Bennett, M.V., et al., Physiology and ultrastructure of electrotonic junctions. II. Spinal and medullary electromotor nuclei in mormyrid fish. Journal of Neurophysiology, 1967. 30(2): p. 180-208.

7. Bullock, T., et al., Electric Organs and Their Control, in Electroreception. 2005, Springer New York. p. 410-451.

8. Aguilera, P.A., M.E. Castello, and A.A. Caputi, Electroreception in Gymnotus carapo: differences between self-generated and conspecific-generated signal carriers. J Exp Biol, 2001. 204(Pt 2): p. 185-98.

9. Black-Cleworth, P., The role of electrical discharges in the non-reproductive social behaviour of Gymnotus carapo (Gymnotidae, Pisces). Animal Behaviour Monographs, 1970. 3: p. 1IN1-77.

10. Stoddard, P.K., et al., Circadian rhythms in electric waveform structure and rate in the electric fish Brachybypopomus pinnicaudatus. Physiol Behav, 2007. 90(1): p. $11-20$.

11. Silva, A., R. Perrone, and O. Macadar, Environmental, seasonal, and social modulations of basal activity in a weakly electric fish. Physiol Behav, 2007. 90(2-3): p. 525-36.

12. Jun, J.J., A. Longtin, and L. Maler, Enhanced sensory sampling precedes self-initiated locomotion in an electric fish. J Exp Biol, 2014. 217( $\mathrm{Pt} 20)$ : p. 3615-28.

13. Richer-de-Forges, M.M., W.G.R. Crampton, and J.S. Albert, A New Species of Gymnotus (Gymnotiformes, Gymnotidae) from Uruguay: Description of a Model Species in Neurophysiological Research. Copeia, 2009. 2009(3): p. 538-544.

14. Migliaro, A., Modulación ambiental y bormonal del ritmo circadiano de la conducta eléctrica, in PEDECIBA. 2018, Universidad de la Republica, Uruguay.

15. Migliaro, A. and A. Silva, Melatonin Regulates Daily Variations in Electric Behavior Arousal in Two Species of Weakly Electric Fish with Different Social Structures. Brain Behav Evol, 2016. 87(4): p. 232-41. 
16. Vitar, M., El Núcleo Marcapaso de Gymnotus omarorum: Caracterización de la Variabilidad de un Modelo de Oscilador Neural en Vertebrados., in Facultad de Ciencias. 2019, Universidad de la República.

17. Silva, A., et al., Biogeography and Breeding in Gymnotiformes from Uruguay. Environmental Biology of Fishes, 2003. 66(4): p. 329-338.

18. Chen, B., et al., Insights into Entropy as a Measure of Multivariate Variability. Entropy, 2016. 18(5): p. 196.
19. Gómez-Laplaza, L.M. and E. Morgan, Effects of short-term isolation on the locomotor activity of the angelfish (Pterophyllum scalare). Journal of Comparative Psychology, 1991. 105(4): p. 366.

20. Pasquarelli, N., et al., Effect of a change in housing conditions on body weight, behavior and brain neurotransmitters in male C57BL/6J mice. Behavioural brain research, 2017. 333: p. 35-42. 


\section{Electrocortical temporal complexity during wakefulness and sleep: an updated account}

Joaquín González ${ }^{1}$

Matias Cavelli ${ }^{1,2}$

Alejandra Mondino ${ }^{1,3}$

Claudia Pascovich ${ }^{1}$

Santiago Castro-Zaballa ${ }^{1}$

Nicolás Rubido ${ }^{4,5}$

Pablo Torterolo ${ }^{1^{*}}$

\footnotetext{
${ }^{1}$ Departamento de Fisiología, Facultad de Medicina, Universidad de la República, Montevideo, 11200, Uruguay.

${ }^{2}$ Department of Psychiatry, University

of Wisconsin, Madison, WI, USA.

${ }^{3}$ Department of Anesthesiology,

University of Michigan, Ann Arbor, MI, USA.

${ }^{4}$ Instituto de Física de Facultad de Ciencias, Universidad de la República, Montevideo, 11400, Uruguay.

${ }^{5}$ Institute for Complex Systems and Mathematical Biology, University of Aberdeen, Aberdeen, AB24 3UE, United Kingdom.
}

\begin{abstract}
The states of sleep and wakefulness are critical physiological processes associated with different brain patterns of activity. The intracranial electroencephalogram allows us to measure these changes, thus, it is a critical tool for its study. Recently, we showed that the electrocortical temporal complexity decreased from wakefulness to sleep. Nevertheless, the origin of this complex activity remains a controversial topic due to the existence of possible artifacts contaminating the brain signals. In this work, we showed that complexity decreases during sleep, independently of the electrode configuration employed. This fact strongly suggests that the basis for the behavioral-state differences in complexity does not have an extracranial origin; i.e., generated from the brain.
\end{abstract}

Keywords: NREM; REM; Entropy; Ordinal Patterns 


\section{INTRODUCTION}

The sleep-wake cycle is a critical physiological process and one of the most preserved biological rhythms through evolution. It is composed by the states of wakefulness (W), nonrapid eye movement (NREM) sleep, and rapid eye movement (REM) sleep ${ }^{1}$. These states are associated with different dynamical patterns of electric activity, which can be recorded accurately through the intracranial electroencephalogram, also known as electrocorticogram (ECoG).

In our previous work ${ }^{2}$, we found that the ECoGs' temporal complexity decreased from wakefulness to sleep; i.e., the repertoire of dynamical motifs was reduced when the animals fell asleep (Figure 1A, B and C). Interestingly, we observed this result in several cortical locations independent of its function (motor, olfactory, somatosensorial and visual), which suggested that the loss in temporal complexity was a global motif developed in the passage from W to sleep. Nevertheless, whether this result originated because a genuine change in brain dynamics happened or was a consequence of an artefactual measurement common to all recording electrodes, remained unanswered. It is important to consider this possibility because our previous recordings ${ }^{2}$ employed a common reference in the cerebellum, which is in close proximity to the neck muscles and could be contaminated by the muscular activity.

In order to discard this possibility, we re-referenced our data to obtain bipolar recordings, and then we measured their temporal complexity employing the same method as our previous work. Therefore, this approach removes the influence of the reference electrode and all common signals from our ECoGs, allowing us to investigate whether our previous results arise from a common background noise or were truly reflecting a global neural pattern which shifted from W to sleep.

\section{MATERIAL AND METHODS}

In this report, we re-analyzed our previous data, therefore, the methods will be explained briefly and should be consulted $i^{2}$ for a detailed description. We employed $12 \mathrm{Wistar}$ adult rats maintained in a $12 \mathrm{~h}$ light/dark cycle. All experimental procedures
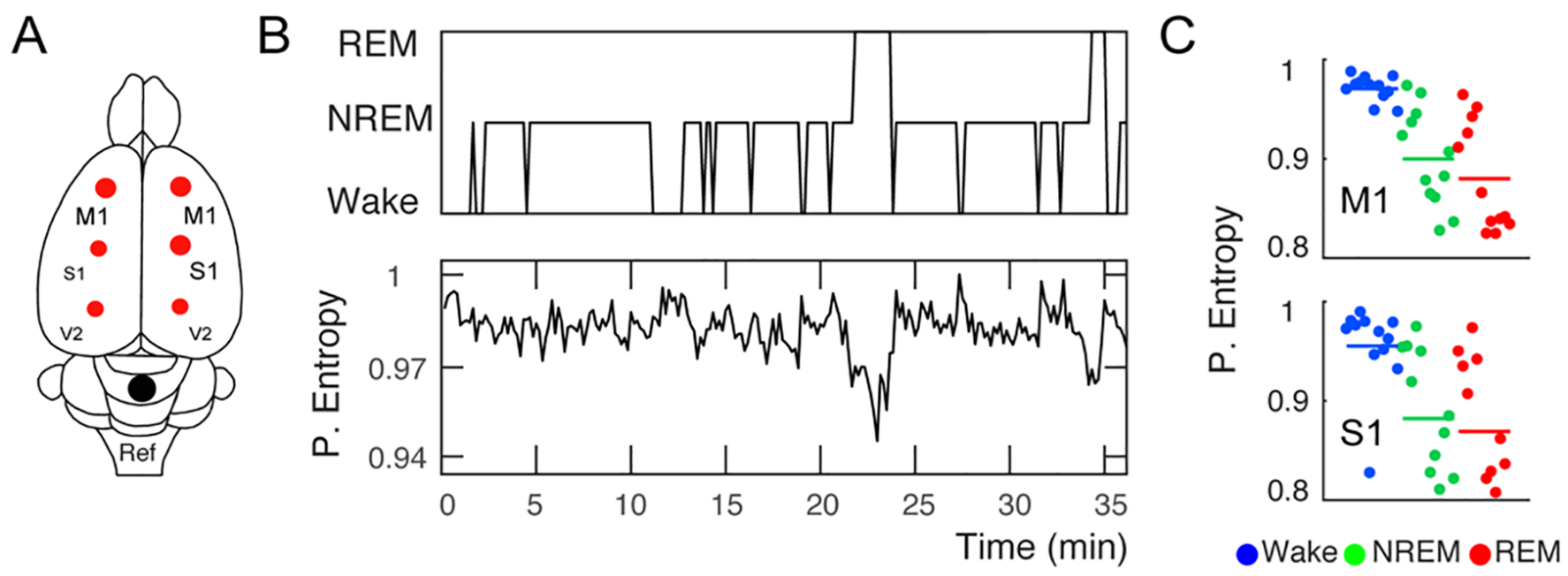

D

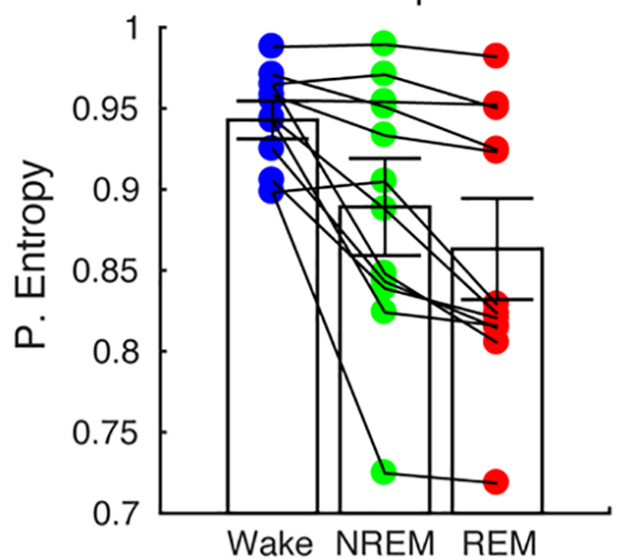

Intrahemispheric

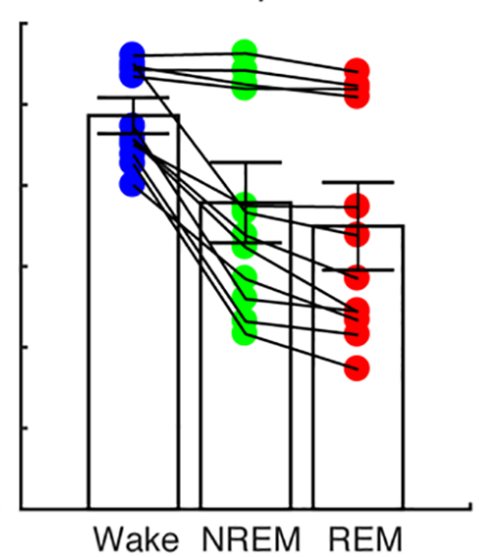

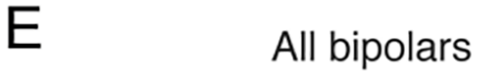

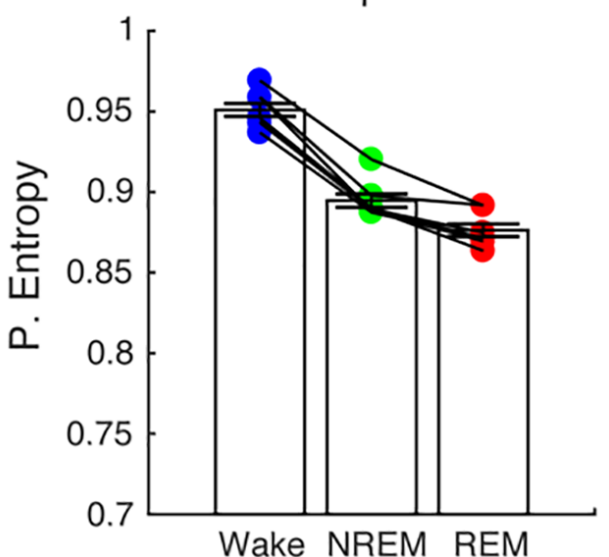

Figure 1. The ECoGs temporal complexity is independent of the electrode configuration. A Electrode localization in the rats cortex. The primary motor (M1; $r$ and l, right and left) and right somatosensory (S1) cortex are shown together with the reference electrode placed above the cerebellum. B The hypnogram (top) from a representative animal is plotted simultaneously with the permutation entropy (bottom) from the M1r cortex as a function of time. C Scatter plots showing the time-average permutation entropy for each animal (12 rats) in each sleep state, blue W, green NREM and red REM. D The same scatter plots are now shown obtained from the bipolar configuration, interhemispheric (M1r-M1l) and intrahemispheric (M1r-S1r). E Permutation entropy decreases through sleep in all the bipolar configurations studied. Each dot depicts a bipolar electrode in each sleep and wake state (averaged from all the animals). 7 bipolars are plotted: M1r-M11, M1r-S1r, M11-S11, S1r-S11, S1r-V2r, S11-V21, V2r-V21. 
were conducted in agreement with the National Animal Care Law (No. 18611) and with the "Guide to the care and use of laboratory animals" (8th edition, National Academy Press, Washington DC, 2010). Furthermore, the Institutional Animal Care Committee (Comisión de Ética en el Uso de Animales) approved the experiments (Exp. No 070153-000332-16).

The animals were chronically implanted with electrodes to monitor the states of sleep and W. To record the ECoG, stainless steel screw electrodes were placed on the skull above motor (bilateral), somatosensory (bilateral), visual cortices (bilateral), the right olfactory bulb, and cerebellum, which was the reference electrode (see Fig. 1a and Table 2 in González et al. 2019). A neck bipolar electrode was employed to record the EMG. Experimental sessions were conducted during the light period, between $10 \mathrm{AM}$ and 4 PM in a sound-attenuated chamber with Faraday shield. The recordings were performed through a rotating connector, to allow the rats to move freely within the recording box. Polysomnographic data were amplified $\mathrm{x} 1000$, acquired and stored in a computer using Dasy Lab Software employing 1024 as a sampling frequency and a 16 bits $\mathrm{AD}$ converter. The states of sleep and $\mathrm{W}$ were determined in $10 \mathrm{~s}$ epochs. W was defined as low voltage fast waves in the motor cortex, a strong theta rhythm $(4-7 \mathrm{~Hz})$ in the visual cortices, and relatively high EMG activity. NREM sleep was determined by the presence of high voltage slow cortical waves together with sleep spindles in motor, somatosensory, and visual cortices associated with a reduced EMG amplitude; while REM sleep as low voltage fast frontal waves, a regular theta rhythm in the visual cortex, and a silent EMG except for occasional twitches. An additional visual scoring was performed to discard artifacts and transitional states.

To assess the ECoGs temporal complexity, we employed the measure known as Permutation Entropy, which has been employed widely ${ }^{3-7}$. This metric is robust to noise and it is computationally efficient. The permutation entropy is calculated as follows: we encoded the ECoGs time-series into ordinal patterns (OPs) by dividing the time-series into sequences of non-overlapping vectors (each containing 3 time stamps), and

A

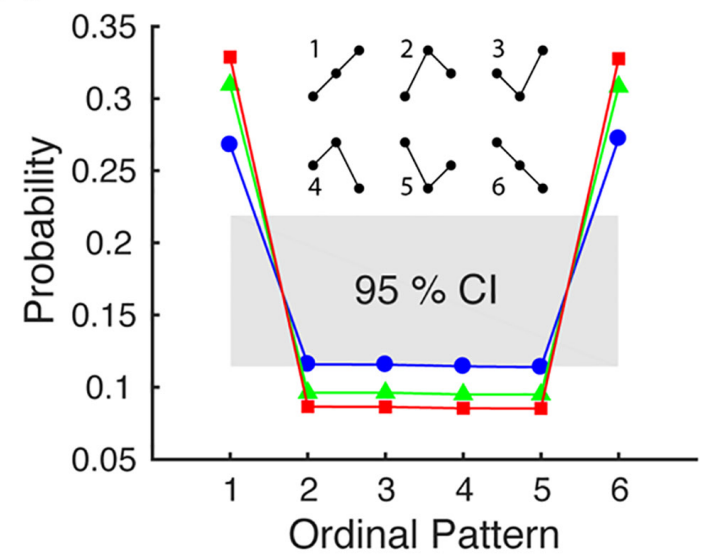

classifying them according to the relative magnitude of its elements. This transforms the graded ECoG time-series into a symbolic one, which can only contain up to six symbols maximum (factorial of the number of elements in a vector). Each symbol then represents a dynamical motif found in the ECoGs.

We note that small noise fluctuations are always introduced into the time-series in order to remove degeneracies; i.e., avoid the cases where, for example $x(t)=x(t+1)$. After the symbolic timeseries is obtained, the permutation entropy is calculated applying the Shannon Entropy ${ }^{8}\left(\mathrm{SE}=-\sum p(\alpha) \log [p(\alpha)]\right)$ to the probability distribution. Where $p(\alpha)$ is the probability (relative frequency of alpha in the symbolic time-series) of the $\alpha$ symbol. For the statistical analysis, we employed the repeated measures ANOVA and set $\mathrm{p}<0.05$ to be considered significant.

\section{RESULTS}

In order to discard the contribution of extracranial noise to the complexity decrease during sleep, we generated bipolar recordings by subtracting two active electrodes. As our original data came as a differential recording to a common reference, the bipolar configuration eliminates the contribution of this electrode ${ }^{9-11}$, in our case, the cerebellum. This is especially important because of the close proximity between our reference electrode and the neck muscles.

Figure 1D shows the results we obtained employing two anatomically relevant configurations: one an interhemispheric (M1r - M1l) and the other an intrahemispheric (M1r - S1r) combination. When we analyzed this new data, we found that the temporal complexity still decreased from W to NREM sleep and reached its lowest values during REM sleep. Furthermore, this result was observed in all the bipolar recordings analyzed (Figure 1D), irrespective of being inter or intrahemispheric combinations; notice that the complexity decrease during sleep is seen in every bipolar configuration employed (Figure $1 \mathrm{E})$. When we investigated the origin of this complex activity, we found that the predominant temporal patterns were the monotonically increasing or decreasing ones (Figure 2A).

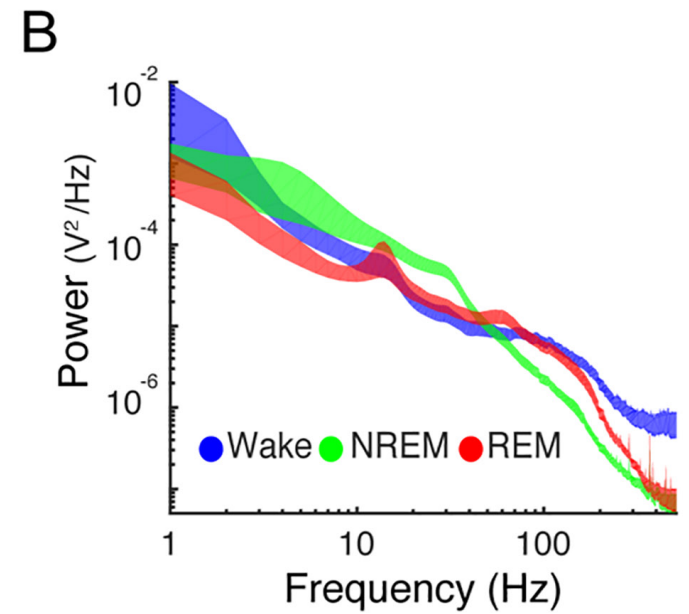

Figure 2. The dynamical characteristics of the ECoGs are preserved in the bipolar configuration . A Ordinal pattern probability distribution from the interhemispheric combination (M1r-M1l). The shaded area depicts the 95 percent confidence interval of the mean. The color code employed is the same as in panel B. B Average power spectral density (12 animals) during wakefulness and sleep, for the M1r-M11 bipolar configuration. The shaded areas depict the mean +/- the standard error. 
This happened during $\mathrm{W}$ and was further overexpressed during sleep. It is worth noting that the frequency distribution of the bipolar ECoGs showed a power-law distribution which was steepened during the sleep states (Fig.2B), similar to our previous result found in monopolar electrodes (see Figure 2.B in (2)).

\section{DISCUSSION}

In the present study, we show that the loss in temporal complexity during sleep is not a consequence of a common noise entering through our reference electrode. This was evidenced by generating bipolar recordings, thus severely reducing the background noise common to all ECoG electrodes ${ }^{9-12}$. This is particularly relevant because our reference electrode was closely located to the neck muscles and thus could be contaminated by the changes in muscle tone during sleep. In contrast, all bipolar recordings showed a significant complexity decrease as sleep progressed and reached its lowest values during REM. This means that our initial findings were independent on the electrode configuration employed (bipolar vs monopolar), and are less likely to simply reflect the changes in muscle activity during the sleep-wake cycle. Furthermore, the bipolar recordings retained a similar frequency and ordinal pattern distribution to what we had observed by the monopolar configuration, implying that these new changes in complexity arise from the same dynamic profile as in the monopolar case. Taken together, our results confirm that the electrocortical temporal complexity decreases from $\mathrm{W}$ to sleep, and this fact is not a consequence of a muscle artifact recorded through the reference electrode.

\section{REFERENCES}

1. Carskadon MA, Dement WC. Chapter 2 - Normal Human Sleep: An Overview. 2011;21.

2. González J, Cavelli M, Mondino A, Pascovich C, Castro-Zaballa S, Torterolo P, et al. Decreased electrocortical temporal complexity distinguishes sleep from wakefulness. Sci Rep. 2019 Dec 5;9(1):18457.

3. Bandt C, Pompe B. Permutation entropy: a natural complexity measure for time series. Phys Rev Lett. 2002 Apr 29;88(17):174102.

4. Bandt C. A New Kind of Permutation Entropy Used to Classify Sleep Stages from Invisible EEG Microstructure. Entropy. 2017 May;19(5):197.

5. Parlitz U, Berg S, Luther S, Schirdewan A, Kurths J, Wessel N. Classifying cardiac biosignals using ordinal pattern statistics and symbolic dynamics. Comput Biol Med. 2012 Mar 1;42(3):319-27.

6. Keller K, Unakafov AM, Unakafova VA. Ordinal Patterns, Entropy, and EEG. Entropy. 2014 Dec;16(12):6212-39.

7. Amigó JM, Keller K, Unakafova VA. Ordinal symbolic analysis and its application to biomedical recordings. Philos Trans R Soc Math Phys Eng Sci. 2015 Feb 13;373(2034):20140091.

8. Shannon CE. A Mathematical Theory of Communication. Bell Syst Tech J. 1948;27(3):379-423.

9. Nunez PL, Srinivasan R. Electric fields of the brain: the neurophysics of EEG. 2nd ed. Oxford; New York: Oxford University Press; 2006. $611 \mathrm{p}$.

10. Nunez PL, Srinivasan R, Westdorp AF, Wijesinghe RS, Tucker DM, Silberstein RB, et al. EEG coherency: I: statistics, reference electrode, volume conduction, Laplacians, cortical imaging, and interpretation at multiple scales. Electroencephalogr Clin Neurophysiol. 1997 Nov 1;103(5):499-515.

11. Nunez PL, Silberstein RB, Shi Z, Carpenter MR, Srinivasan R, Tucker DM, et al. EEG coherency II: experimental comparisons of multiple measures. Clin Neurophysiol Off J Int Fed Clin Neurophysiol. 1999 Mar;110(3):469-86.

12. Li G, Jiang S, Paraskevopoulou SE, Wang M, Xu Y, Wu Z, et al. Optimal referencing for stereo-electroencephalographic (SEEG) recordings. NeuroImage. 2018 Dec 1;183:327-35. 


\section{Fetal programming of adipose tissue function by gestational chronodisruption}

Diego Halabi ${ }^{1,4}$

Pamela Ehrenfeld 1,2,3,5

Natalia Mendez ${ }^{1,3}$

Georg Hans Richter Hans ${ }^{1,3}$

Claudia Torres-Farfan ${ }^{1,3,5^{*}}$

\footnotetext{
${ }^{1}$ Laboratory of Developmental Chronobiology, Faculty of Medicine, Universidad Austral de Chile, Valdivia, Chile.

${ }^{2}$ Laboratory of Cellular Pathology, Institute of Anatomy, Histology and Pathology, Universidad Austral de Chile, Valdivia, Chile.

${ }^{3}$ Institute of Anatomy, Histology and Pathology, Universidad Austral de Chile, Valdivia, Chile.

${ }^{4}$ Institute of Dentistry, Universidad Austral de Chile, Valdivia, Chile.

${ }^{5}$ Centro Interdisciplinario de Estudios del Sistema Nervioso (CISNe),

Universidad Austral de Chile, Valdivia, Chile.
}

\begin{abstract}
The interaction of the maternal and fetal circadian systems is recognized as a crucial crosstalk for fetal development, which may be a key factor determining fitting health in adulthood. Unfortunately, in our modern societies the relevance of regular light/dark cycles has been dangerously disregarded, generating a disarray of the internal temporal order of circadian physiological functions (i.e. chronodisruption). In fact, it is well established that gestational circadian misalignment negatively affects gestation progression; probably through alteration of maternal melatonin transfer to the fetus, given that melatonin is inhibited by light at night, therefore depriving the fetus of a maternal light/dark signal. Mounting evidence supports that the intermittent circadian rhythm of melatonin induced by gestational chronodisruption provides an anomalous adaptive frame to the fetus, programming abnormal metabolic function in the adult. Indeed, adult male offspring gestated under chronodisruption displays a circadian disarray and several metabolic abnormalities, like increased body weight and adipose tissue mass, together with abnormal responses to glucose and insulin; suggestive of white adipose tissue dysfunction. Thus, gestational chronodisruption is akin to other human and animal models of Developmental Origin of Health and Disease.

In the current review we will discuss the role played by maternal circadian rhythms in fetal development and the impact of fetal-maternal desynchronization on offspring's health and disease; with special focus on metabolic disorders and the role played by adipose tissue in the metabolic unbalance induced by chronodisruption.
\end{abstract}

Keywords: Chronodisruption; Fetal Programming; Obesity; Adipose Tissue; Dohad; Circadian Rhythms

\author{
Corresponding author: \\ Claudia Torres-Farfan \\ E-mail: claudia.torres@uach.cl
}




\section{INTRODUCTION}

The World Health Organization defines obesity as a condition in which body mass index is equal to or greater than $30^{1}$. It is one of the leading public health issues in the whole world, with the incidence of obesity being duplicated from 1980, and more than 200 million men and nearly 300 million women were classified as obese in $2008^{1}$. In addition, obesity is strongly associated with many concomitant diseases, such as type 2 diabetes, insulin resistance, hypertension, cardiovascular disease and some types of cancer ${ }^{2,3}$. The direct economic cost linked to obesity in USA is between $0.7 \%$ and $2.8 \%$ of the total health expenditure in the country, with medical costs $30 \%$ higher in obese individuals compared to those with a healthy weight ${ }^{4}$. In Chile, according to the National Health Survey 2016$17^{5}$, there are $39.8 \%$ overweight and $31.2 \%$ obese adults, which is on an upward trend compared to the National Health Survey conducted in 2006 (39.3\% and $25.1 \%$ respectively) and 2003 $\left(37.8 \%\right.$ and $23.2 \%$ respectively) ${ }^{6}$. Obesity is clinically observed as an abnormal and excessive accumulation of adipose tissue, linked to a state of chronic low-grade inflammation?

Current strategies to treat or prevent overweight and obesity, with their related complications, are focused on managing postnatal lifestyles; exercise, balanced sugar consumption and healthy diet. These interventions, although individually seem to be effective, have questionable success as public health measures, so it is not surprising that in industrialized countries there is a steady increase in obesity rates ${ }^{8}$. This leads to the exploration of new strategies for prevention and management of obesity, focusing on the etiology.

There is strong evidence supporting that gestational chronodisruption has negative effects on the offspring, as reported by us and others in animal models ${ }^{9-16}$. Thus, gestational chronodisruption is akin other human and animal models of Developmental Origen of Health and Disease (DOHaD) ${ }^{17-21}$. In the current review we will discuss the role played by maternal circadian rhythms on fetal development, and the impact of fetalmaternal desynchronization on offspring's health and disease; with special focus on metabolic disorders and the role played by adipose tissue in the metabolic unbalance induced by chronodisruption.

\section{Adipose tissue, a white and brown balance}

White adipose tissue (WAT) is the main responsible for energy storage as triglycerides and plays an important endocrine function through secretion of hormones and pro-inflammatory cytokines, while the accumulation of WAT is directly related to obesity onset. White adipocytes are spherical cells of highly variable size, which is determined by a single lipid droplet pushing the nucleus towards the cell membrane and occupying about $90 \%$ of the cell volume, while the mitochondria are thin and elongated ${ }^{22}$. In mammals, there are different WAT depots distributed throughout the whole body, and the accumulation/ pathogenicity varies depending on each depot. The most harmful is the accumulation of abdominal subcutaneous adipose tissue, while some visceral depots even act as protective factors ${ }^{23}$.
Besides its role in energy storage, WAT is also an active secretory organ producing many molecules termed adipokines. Adipokines participate in the modulation of glucose and lipid homeostasis via central effects of leptin. Furthermore, adipokines include proinflammatory factors and chemokines, which are increased in obesity. Although obesity has been associated with increased accumulation of macrophages within fat mass and WAT in rodents, it remains unclear how the crosstalk between macrophages and white adipocytes triggers dysfunction of these cells in metabolic disorders. Recently, it has been demonstrated that metabolic syndrome and chronodisruption are linked by early onset of lowgrade inflammation ${ }^{24}$. Chronic low-grade inflammatory state is a pathological feature of a wide range of chronic conditions, such as the metabolic syndrome, type 2 diabetes mellitus and cardiovascular disease ${ }^{25}$. Interestingly, adipose tissue hypertrophy is associated with immune cell infiltration, in particular that of macrophages and $\mathrm{T}$ cells, and a local proinflammatory milieu wherein key cytokines including TNF- $\alpha$, IL-6, IL-1 $\beta$ IL-10 and C reactive protein (CRP), associated with insulin resistance ${ }^{26}$ (Figure 1).

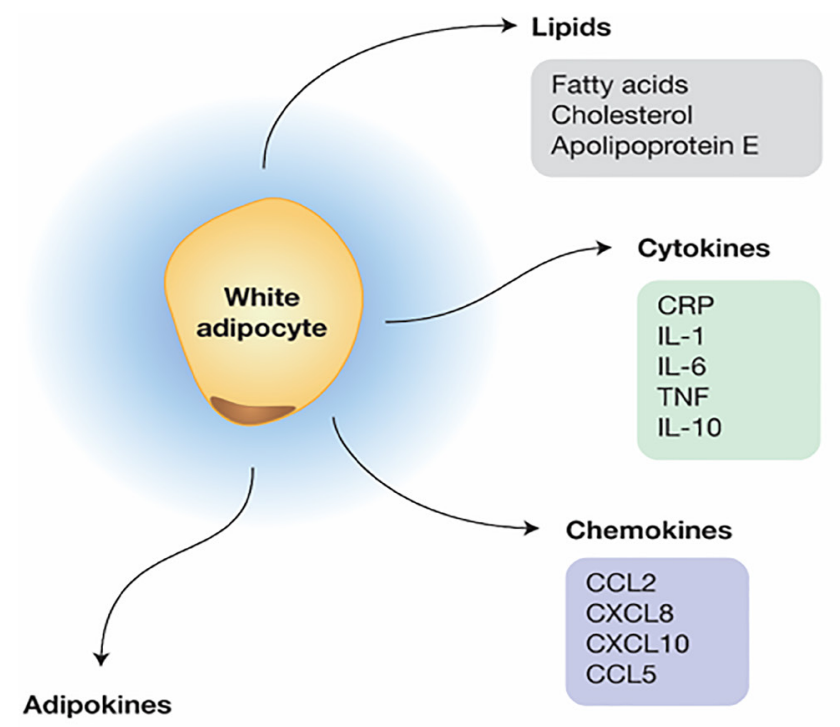

\begin{tabular}{|l|l|}
\hline Resistin & Visfatin \\
$\begin{array}{l}\uparrow \text { Insulin resistance } \\
\uparrow \text { Inflammation } \\
\text { Obesity }\end{array}$ & $\downarrow$ Plasma glucose \\
& $\downarrow$ Inflammation \\
& Adipocyte maturation \\
Leptin & Adiponectin \\
$\begin{array}{l}\downarrow \text { Insulin sensitivity } \\
\downarrow \text { Hungry }\end{array}$ & $\uparrow$ Insulin Sensitivity \\
$\uparrow$ Inflammation & $\downarrow$ Inflammation \\
Type II diabetes mellitus & Other \\
Atherosclerosis & Apelin \\
& Vaspin \\
& Omentin \\
& Hepcidin \\
\hline
\end{tabular}

Figure 1. The multiple endocrine functions of white adipose tissue (WAT). The WAT functions include adipokine synthesis and secretion, lipid uptake, storage and synthesis, glucose homeostasis and inflammation status. Leptin, resistin and adiponectin are named adipokines, because are only synthetized and released by adipocytes. Abbreviations: CRP, C-reactive protein; IL, interleukin; TNF, tumor necrosis factor; CCL, CC-chemokine ligand; CXCL, C-X-C motif chemokine ligand. 
On the other hand, brown adipose tissue (BAT) is structurally composed by brown adipocytes, which are polyhedral cells with multiple lipid droplets of small size (compared to white adipocytes), and have numerous mitochondria, large and spherical. The vascularization of BAT is higher than WAT, which together with the great density of mitochondria gives it the characteristic brown $\operatorname{color}^{27}$. The only known function of BAT is the dissipation of energy as heat, which is produced from triglycerides stored in brown adipocytes, a physiological process called thermogenesis ${ }^{28,29}$.

Until 15 years ago, BAT was considered to disappear after birth, since it generates a great thermogenic activity to protect the human newborn from hypothermia. Therefore, for a long time BAT seemed an unimportant organ for adult metabolism. But the recent discovery of active depots of BAT in adults by positron emission tomography/computed tomography (PET/CT) ${ }^{30}$ has renewed the interest in the potential relevance of this tissue for adult metabolism. Through these studies, anatomical locations have been determined mainly in the interscapular and cervicalsupraclavicular region, observed throughout all age ranges in humans ${ }^{31}$ and rodents ${ }^{32}$.

BAT activation (thermogenesis) is mainly regulated by the stimulation of noradrenaline, which activates the signaling of $\beta 3$ Adrenoceptors located in the plasma membrane of mature brown adipocytes (Figure 2). The activation of this receptor increases the cytoplasmic levels of cAMP, which activates protein kinase A, which in turn phosphorylates the enzyme hormone-sensitive lipase (HSL) and perilipin. HSL induces the lipolysis of triglycerides in fatty acids (essential substrate for thermogenesis) and glycerol, which can be measured as an indirect marker of lipolysis ${ }^{33}$.

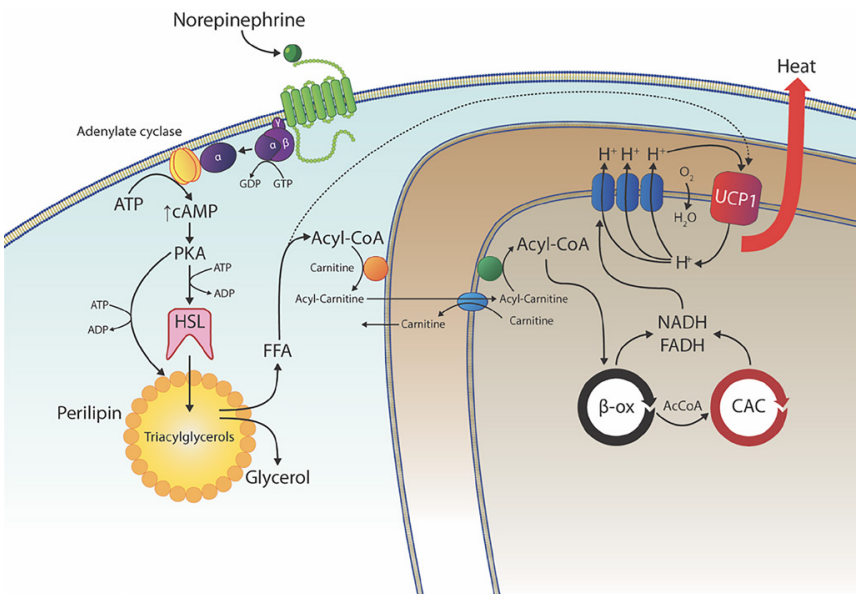

Figure 2. Schematic representation of thermogenesis mechanism in a brown adipocyte. Stimulation of $\beta 3$-adrenergic receptors by norepinephrine from the nerves of the sympathetic nervous system triggers an intracellular signaling pathway that breaks the lipid droplets in the adipocyte (lipolysis) by phosphorylation of the hormone-sensitive lipase (HSL), and mobilizes the fatty acids (FFA) released into the mitochondria These triglycerides are $\beta$-oxidized ( $\beta$-ox), releasing acetyl-CoA that enters the citric acid cycle (CAC). The mitochondria of the brown adipocytes are unique by expressing the uncoupling protein 1 (UCP1) in the inner membrane, which allows the respiratory chain to be uncoupled, pumping back the protons of the intermembrane space. This nonsense process generates heat (thermogenesis).
On the other hand, perilipin protects these triglycerides located in lipid drops from the adipocyte activity of HSL, and upon phosphorylation, it is dissociated from these triglycerides, leaving them exposed. Therefore, lipolysis induced by noradrenaline is essential for thermogenesis; while any mechanism inducing lipolysis in brown adipocytes leads to thermogenesis; conversely, thermogenesis does not occur if lipolysis is inhibited ${ }^{22}$.

Because of lipolysis, most of the fatty acids remain in the cytosol, coupled to binding proteins such as A-FABP (adipocyte form of the fatty acid-binding protein) or H-FABP (heart form of the fatty acid-binding protein). Whereas a lower fraction of fatty acids is transported to the peroxisome, most are transported to mitochondria, which are abundant in brown adipocytes. In this cell compartment fatty acids are $\beta$-oxidized, releasing acetyl-CoA and entering the citric acid cycle. As in other mitochondria of any cell type, electrons flux released through the respiratory chain results in proton pumping from the mitochondrial matrix, establishing a membrane potential that results in the production of ATP. Interestingly, brown adipocytes are the distinctive cell type possessing UCP1 (uncoupling protein 1), which uncouples the respiratory chain and returns the protons to the mitochondrial matrix, generating large amounts of energy that is released as heat (thermogenesis).

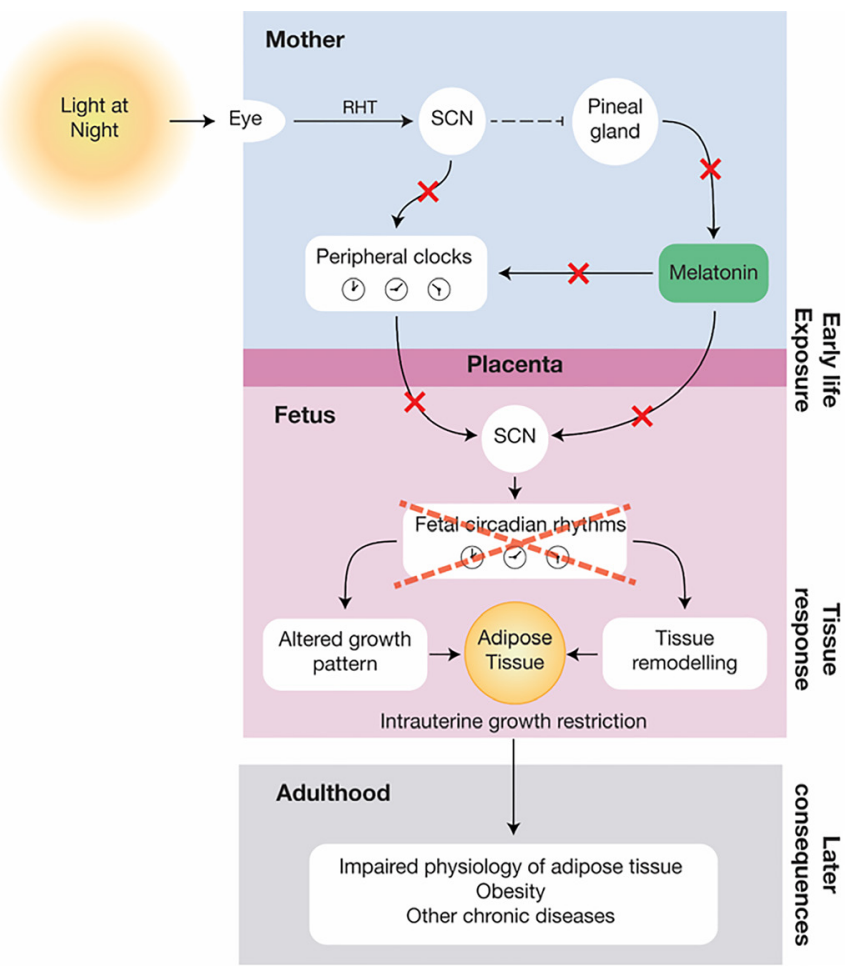

Figure 3. A hypothetical schematic representation of fetal programming in adult adipose tissue induced by gestational chronodisruption. Exposure of a pregnant mother to light at night commands her suprachiasmatic nucleus $(\mathrm{SCN})$, through the retinohypothalamic tract (RHT), to inhibit the production of melatonin in the pineal gland. Since the fetus is unable to produce its own melatonin and fetal SCN is not functional, it cannot entrain fetal peripheral clocks. Whereas the mechanisms are not clear, peripheral tissues modify and remodel their growth pattern in order to allow the development of vital organs such as the brain, a tradeoff know as intrauterine growth restriction (IUGR). As a consequence, there is a higher risk of obesity at adulthood due to adipose tissue programming. 
This senseless use of stored fatty acids seems to be beneficial in the control of obesity, as BAT can dissipate excess of energy as heat ${ }^{34}$. Indeed, obesity is characterized by an increased white/ brown adipose tissue ratio compared to lean subjects, while the increase of BAT activity is positively associated with wholebody energy expenditure ${ }^{35,36}$.

\section{Adipose tissue development}

Compared to WAT, development of BAT begins early (by mid-gestation) and it persists throughout life in a continuous process $^{37}$. Although both tissues are fatty tissues, the origin of WAT and BAT are completely different.

White adipocytes are originated from adipocyte precursors (or pre-adipocytes), which derive from mesenchymal stem cells (MSCs) and differentiate into mature and functional adipocytes $^{38}$. The development of human fetal adipose tissue was schematically divided into 5 morphogenic phases (Poissonnet et al., 1983) that occur between weeks 14 and 23 of gestation: (A) the emergence of loose connective tissue, (B) appearance of aggregates of mesenchymal cells surrounding proliferating vessels, (C) mesenchymal cells differentiating into pre-adipocytes, (D) appearance of lipid droplets in the adipocyte cytoplasm surrounding capillaries, and (E) lobes of adipose tissue with clearly differentiated adipocytes. This process is guided by the expression of various transcription factors, highlighting the $\operatorname{PPR} \gamma$ (peroxisome proliferator-activated receptor- $\gamma$ ); PPR $\delta$; C/ $\mathrm{EBP} \alpha, \beta, \gamma$ (CCAAT/enhancer-binding proteins) and SREBP1 (sterol regulatory element-binding protein 1 ), in addition to the expression of specific enzymes that metabolize lipids, such as FAS $^{39-42}$.

In contrast, brown adipocytes share a common origin with muscle cells. Since both cell types are specialized in lipid catabolism but not in their storage, both are innervated by the sympathetic nervous system, contain a high density of mitochondria, and participate in thermogenesis ${ }^{43}$. In rats, brown pre-adipocytes are derived from mesenchymal cells. These precursors are organized into lobes separated by septa of connective tissue, which begin to accumulate lipids and display an unilocular shape very similar to white adipocytes. Later, these cells accumulating a higher amount of lipids take on their characteristic multilocular morphology. In rats, BAT can be observed from 15 to 17 days of gestation and continue to develop throughout whole life ${ }^{44}$. In humans, brown adipose tissue can be observed from gestational week 23 in the cervical, thoracic and peripheral areas to abdominal viscera ${ }^{45}$.

\section{Is chronodisruption a critical risk factor for obesity?}

Almost all our physiological, metabolic, endocrine and behavioral processes are influenced by internal, daily and recurrent rhythms -named circadian rhythms- that represent an adaptation to the day/night cycle. However, over the last century, disorganized and even constant artificial light schedules have become a dominant feature in or modern industrial and technological society.
This is very different from the natural light conditions to which humans have been exposed throughout most humankind evolution, which were governed almost exclusively by the 24-hour oscillations of day and night. This artificial condition has been named "chronodisruption", which leads to a significant alteration of the temporal organization of endocrinology, physiology, metabolism and behavior ${ }^{46,47}$. In other words, exposure to artificial light at night disorganizes our internal biological clocks, with consequences that we are just beginning to know.

Shift work schedule is one of the most frequent ways of exposure to chronodisruption. In 2006 there were 931 articles indexed in PubMed for the term "shift work" 2020, 3930 articles were published. This significant number of scientific publications, both epidemiological and basic research, allow us to asseverate that the alteration of the circadian system -which occurs, for example, in work under rotating shift schedules-contributes to the development of several pathologies associated with modern life, including obesity ${ }^{49}$.

To understand why this happens, we must delve into the circadian system that regulates our internal clocks, that are susceptible to desynchronization by chronodisruption. There are different external signals that entrain (synchronize) the biological clocks that regulate the periodicity of the circadian system, for example; food intake, social cues, etc. The most important external signal in mammals is the light perceived by the retina, which signals to the hypothalamic suprachiasmatic nucleus (SNC) in the anterior hypothalamus through the optic nerve carries. In turn, the SCN regulates different peripheral clocks in practically all the organs of the body, giving rise to temporal cues synchronizing the rhythms of diverse biological functions throughout the body. The SNC projects neurons to the paraventricular nucleus, which projects neurons to the lateral intermediate nucleus of the spinal cord (thoracic portion) and, through the superior cervical ganglion, communicates with the pineal gland that secretes the hormone melatonin. Melatonin is secreted with a characteristic circadian pattern, with high levels during the night while almost depleted levels during daylight hours; being an important transducer of the light / dark cycle from the brain to the rest of the whole organism ${ }^{50}$.

Biological rhythms appear very early in development. Studies in humans, non-human primates, and sheep have shown that there are 24-hour circadian rhythms in fetal heartbeat, respiratory movements, and hormones release. However, the fetal and adult circadian systems are different; in the fetus, the SNC is not functional and its pineal gland does not secrete melatonin. However, a circulating fetal melatonin rhythm has been well described, secondary to circulating maternal melatonin crossing the placental barrier and synchronizing peripheral clocks in the developing fetus ${ }^{51}$. The maternal-fetal relationship of the circadian system not only has the function of being a replacement while the fetal SCN develops, but also fulfills the important function of entraining the circadian system of the fetus for an adequate transition to the environmental conditions that he will face at birth. 
Furthermore, sheep studies carried out by our group have shown that melatonin directly inhibits the noradrenaline response in brown adipose tissue ${ }^{52}$. It must be kept in mind that the thermogenic activity would become effective only at birth, when the fetus is transferred from a stable intrauterine temperature to extrauterine variable environmental temperature, coinciding with loss of the rhythmic maternal melatonin stimuli. Hence, the abrupt suppression of maternal melatonin signaling at birth alters the regulation of fetal biological rhythms and physiology, which might have important consequences at this life stage.

Chronodisruption is an emergent risk factor for adipose tissue dysfunction and therefore obesity. In humans, shift work increases 1.3 to 1.5 times the risk of being obese ${ }^{53,54}$, which is directly correlated with the length of the rotating shift work schedule ${ }^{55}$. Analysis of global gene expression in adipose tissue has shown presence of robust 24-hour circadian rhythms. This gene expression rhythmicity is correlated with SNC intrinsic rhythms, indicating an inbuilt activity of the adipose tissue as a peripheral $\operatorname{clock}^{56,57}$. Moreover, mutant mice in which SCN rhythmicity was blunted showed a desynchronization in the expression of clock genes in epididymal tissue, demonstrating its strong dependence on the $\mathrm{SCN}$ as a master pacemaker ${ }^{58}$.

Microarray analysis suggests that more than $20 \%$ of the transcriptome in mouse adipose tissue is expressed under a circadian rhythmic pattern, while the genes displaying stronger differential expression are involved in metabolic pathways ${ }^{5,59}$. Other authors reported similar results using microarray of subcutaneous human white adipose tissue ${ }^{60,61}$. The rhythmicity in human WAT explants was confirmed by Gómez-Santos et al. in $2009^{62}$, who removed visceral and subcutaneous WAT from obese subjects and analyzed them every 6 hours for 24 hours, obtaining results consistent with the rhythms observed in other organs which act as peripheral clocks.

Then, adipose tissue is an organ that works rhythmically, synchronized by light/dark cycles, and therefore susceptible to being altered by chronodisruption. Epidemiological evidence of this statement has an origin in the 50s, when the direct association between nighttime food intake and obesity was reported ${ }^{63}$. Subsequently, several investigations confirmed the direct association between feeding schedules and the risk of weight gain in humans and animal models ${ }^{64-67}$. Studies conducted between the 80s and 90s showed that glucose and insulin metabolism have a 24-hour rhythm in healthy people, as well as in people with diabetes mellitus ${ }^{68-70}$. On the other hand, sleep deprivation was found to increase serum glucose levels and insulin secretion in healthy patients ${ }^{71}$. Moreover, studies in animal models have shown circadian oscillations in mRNA levels that encode for important adipogenic regulators, such as PPAR $\alpha$, SREBP1 and NR1D172-75. Regarding these investigations, endocrine studies in healthy people have determined that serum levels of the enzyme lipoprotein lipase (secreted by adipocytes) and adipokines -such as leptin and adiponectin- display 24-hour circadian oscillations ${ }^{76-81}$. Furthermore, a growing amount of scientific evidence shows that obesity can attenuate the amplitude and change the phase of circadian measurements in adipose tissue function ${ }^{82-84}$.

\section{Adipose tissue can be programmed by maternal chronodisruption}

Several epidemiological and experimental studies support the early origin of adult diseases due to adverse environmental conditions that occurred during fetal life. Gestational nutrition restriction ${ }^{85}$, stress $^{86}$, exposure to drugs for medical or recreational use $^{87}$, and chronodisruption ${ }^{911-13,47}$, have been linked to adverse effects on health at adulthood.

This concept, named "fetal programming," was proposed by David Barker in the 1980s, particularly with nutrient restriction as an insult during early life. In his studies, he observed that a phenotype of cardiovascular disease is programmed in adulthood, and the consequent premature death in response to a pregnancy carried out with nutrient restriction ${ }^{88}$. Fetal programming has been proposed as an adaptive response to an adverse fetal environment, to protect the growth of key organs -such as the brain, for example- in detriment of others -such as adipose tissue- resulting in impaired postnatal metabolism. These studies were the starting point for several clinical, epidemiological and animal studies throughout the world that supported the concept of fetal programming as the origin of numerous adult diseases, such as obesity, insulin resistance, type 2 diabetes, cardiovascular diseases $^{89-92}$ or intrauterine growth restriction (IUGR) ${ }^{93}$.

Early studies showed that exposure to poor nutrition before delivery alters development of adipocytes in utero, resulting in a permanent increase in the ability to form new adipocytes in adipose tissue depots, and increase lipid storage in existing adipocytes ${ }^{94}$. Numerous conditions during pregnancy associated with obesity in adult offspring in humans and rodents have been described, such as caloric restriction ${ }^{95,96}$, maternal obesity and type 2 diabetes ${ }^{97}$.

When men over 65 years old were studied, 30\% of those who were born with $\leq 2.95 \mathrm{~kg}$ had metabolic syndrome, in contrast to $6 \%$ of those born with $\geq 4.31 \mathrm{~kg}^{98}$. Besides, it has been observed that children born with low weight, and who quickly gain the weight considered adequate, have a higher risk of obesity, insulin resistance and hypertension in adulthood ${ }^{98,99}$. There are several lines of evidence linking this association of low weight at birth with the onset of central obesity in adulthood ${ }^{100-102}$, insulin resistance ${ }^{103,104}$ and metabolic syndrome $e^{105,106}$.

A recent observation by our group, indicated that maternal chronodisruption throughout pregnancy induces intrauterine growth restriction in the fetus at gestational day 18, and increases the body weight of newborns ${ }^{9,13}$. Similar results were found when maternal melatonin of pregnant Wistar rats was suppressed by pinealectomy ${ }^{107}$. As mentioned previously, adipose tissue is the main component of weight gain. In addition, we know that maternal melatonin during pregnancy may also play an important role in the development of brown adipose tissue and the thermoregulation of the newborn. Newborns of Capuchin monkeys from mothers chronically exposed to constant light during pregnancy (treatment that suppresses maternal melatonin) had lower body temperature. This was normalized in newborns whose mothers also exposed to constant light received a daily dose of melatonin ${ }^{108}$. 
A series of cases reported by Nahme et al. in $2019^{109}$ suggests that these findings can be extrapolated to human health. These authors, for the first time, observed a direct relationship between misalignment of the melatonin rhythm during pregnancy, and gestational problems together with a low Apgar score in the newborns from these mothers. While we must be careful to overinterpret a series of cases, there are already many studies suggesting an increased risk of adverse pregnancy outcomes secondary to maternal shift work throughout pregnancy ${ }^{110}$, and in their offspring ${ }^{111,112}$.

\section{CONCLUDING PERSPECTIVES}

WAT and BAT play key roles in both, metabolic regulation and obesogenic processes. Surprisingly, there is still limited data accounting for fetal programming of adult disease where these highly specialized tissues may be considered as targets of adverse prenatal conditions, such as gestational chronodisruption. During the last century, this environmental challenge has turned into a main disruptor of normal physiological balance impinging on the large share of the worldwide working force submitted to night shift schedules, including several million pregnant women.

In this context, further research of WAT and BAT is needed to fully unveil the role of rhythmic maternal melatonin transfer to the fetus, chronic low-grade inflammation, circadian and endocrine overt disruption, changes of normal developmental trajectory, functional genomics and epigenomics, among other tissue-specific features. Such an integrative approach would address the actual impact of gestational chronodisruption as a prime candidate affecting fetal WAT and BAT physiology and therefore triggering adult obesity.

\section{REFERENCES}

1. World Health Organization. Obesity and overweight, Fact Sheet. 2016.

2. Prospective Studies Collaboration. Body-mass index and cause-specific mortality in 900000 adults: collaborative analyses of 57 prospective studies. The Lancet. 2009 Mar;373(9669):1083-96.

3. Kahn SE, Hull RL, Utzschneider KM. Mechanisms linking obesity to insulin resistance and type 2 diabetes. Nature. 2006 Dec 14;444(7121):840-6.

4. Tremmel M, Gerdtham U-G, Nilsson P, Saha S. Economic Burden of Obesity: A Systematic Literature Review. International Journal of Environmental Research and Public Health. 2017 Apr 19;14(4):435.

5. Ministerio de Salud, Chile. Encuesta Nacional de Salud 2016-2017. 2017.

6. Ministerio de Salud, Chile. Encuesta Nacional de Salud 2006. 2016.

7. Gregor MF, Hotamisligil GS. Inflammatory mechanisms in obesity. Annu Rev Immunol. 2011;29:415-45.

8. Gluckman PD, Hanson MA. Fat, fate $\&$ disease: why exercise and diet are not enough. Oxford ; New York: Oxford University Press; 2012. 288 p.

9. Mendez N, Halabi D, Spichiger C, Salazar ER, Vergara K, AlonsoVasquez P, et al. Gestational Chronodisruption Impairs Circadian Physiology in Rat Male Offspring, Increasing the Risk of Chronic Disease. Endocrinology. 2016 Dec;157(12):4654-68.

10. Vilches N, Spichiger C, Mendez N, Abarzua-Catalan L, Galdames HA, Hazlerigg DG, et al. Gestational Chronodisruption Impairs Hippocampal Expression of NMDA Receptor Subunits Grin1b/Grin3a and Spatial Memory in the Adult Offspring. Foulkes NS, editor. PLoS ONE. 2014 Mar 24;9(3):e91313.

11. Galdames HA, Torres-Farfan C, Spichiger C, Mendez N, Abarzua-Catalan $\mathrm{L}$, Alonso-Vazquez $\mathrm{P}$, et al. Impact of gestational chronodisruption on fetal cardiac genomics. Journal of Molecular and Cellular Cardiology. 2014 Jan;66:1-11.

12. Spichiger C, Torres-Farfan C, Galdames HA, Mendez N, Alonso-Vazquez P, Richter HG. Gestation under chronic constant light leads to extensive gene expression changes in the fetal rat liver. Physiological Genomics. 2015 Dec;47(12):621-33.
13. Salazar ER, Richter HG, Spichiger C, Mendez N, Halabi D, Vergara K, et al. Gestational chronodisruption leads to persistent changes in the rat fetal and adult adrenal clock and function: Fetal programming of adrenal dysfunction by photoperiod shifting. The Journal of Physiology. 2018 Dec;596(23):5839-57.

14. Richter HG, Mendez N, Abarzua-Catalan L, Valenzuela GJ, SeronFerre M, Torres-Farfan C. Developmental Programming of Capuchin Monkey Adrenal Dysfunction by Gestational Chronodisruption. BioMed Research International. 2018 Aug 13;2018:1-11.

15. Varcoe TJ, Wight N, Voultsios A, Salkeld MD, Kennaway DJ. Chronic Phase Shifts of the Photoperiod throughout Pregnancy Programs Glucose Intolerance and Insulin Resistance in the Rat. Yamazaki S, editor. PLoS ONE. 2011 Apr 6;6(4):e18504.

16. Ferreira DS, Amaral FG, Mesquita CC, Barbosa APL, Lellis-Santos C, Turati AO, et al. Maternal Melatonin Programs the Daily Pattern of Energy Metabolism in Adult Offspring. Yamazaki S, editor. PLoS ONE. 2012 Jun 12;7(6):e38795.

17. Osmond C, Barker DJ. Fetal, infant, and childhood growth are predictors of coronary heart disease, diabetes, and hypertension in adult men and women. Environmental Health Perspectives. 2000 Jun;108(suppl 3):545-53.

18. Fowden AL, Giussani DA, Forhead AJ. Intrauterine Programming of Physiological Systems: Causes and Consequences. Physiology. 2006 Feb;21(1):29-37.

19. Nathanielsz PW. Animal Models That Elucidate Basic Principles of the Developmental Origins of Adult Diseases. ILAR Journal. 2006 Jan $1 ; 47(1): 73-82$.

20. Nuyt AM. Mechanisms underlying developmental programming of elevated blood pressure and vascular dysfunction: evidence from human studies and experimental animal models. Clinical Science. 2008 Jan 1;114(1):1-17.

21. Richter V, Briffa J, Moritz K, Wlodek M, Hryciw D. The role of maternal nutrition, metabolic function and the placenta in developmental programming of renal dysfunction. Clinical and Experimental Pharmacology and Physiology. 2016 Jan;43(1):135-41.

22. Cannon B, Nedergaard J. Brown adipose tissue: function and physiological significance. Physiol Rev. 2004 Jan;84(1):277-359.

23. Karpe F, Pinnick KE. Biology of upper-body and lower-body adipose tissue-link to whole-body phenotypes. Nature Reviews Endocrinology. 2015 Feb;11(2):90-100.

24. Agil A, Reiter RJ, Jiménez-Aranda A, Ibán-Arias R, Navarro-Alarcón M, Marchal JA, et al. Melatonin ameliorates low-grade inflammation and oxidative stress in young Zucker diabetic fatty rats. Journal of Pineal Research. 2013 May;54(4):381-8.

25. Antonelli M, Kushner I. It's time to redefine inflammation. The FASEB Journal. 2017 Mav;31(5):1787-91.

26. Minihane AM, Vinoy S, Russell WR, Baka A, Roche HM, Tuohy KM, et al. Low-grade inflammation, diet composition and health: current research evidence and its translation. British Journal of Nutrition. 2015 Oct 14;114(7):999-1012.

27. Cinti S. Transdifferentiation properties of adipocytes in the adipose organ. American Journal of Physiology-Endocrinology and Metabolism. 2009 Nov;297(5):E977-86.

28. Nedergaard J, Golozoubova V, Matthias A, Asadi A, Jacobsson A, Cannon B. UCP1: the only protein able to mediate adaptive non-shivering thermogenesis and metabolic inefficiency. Biochim Biophys Acta. 2001 Mar 1;1504(1):82-106.

29. Saito M, Okamatsu-Ogura Y, Matsushita M, Watanabe K, Yoneshiro T, Nio-Kobayashi J, et al. High Incidence of Metabolically Active Brown Adipose Tissue in Healthy Adult Humans: Effects of Cold Exposure and Adiposity. Diabetes. 2009 Jul 1;58(7):1526-31.

30. Christensen CR, Clark PB, Morton KA. Reversal of Hypermetabolic Brown Adipose Tissuein F-18 FDG PET Imaging: Clinical Nuclear Medicine. 2006 Apr;31(4):193-6.

31. Leitner BP, Huang S, Brychta RJ, Duckworth CJ, Baskin AS, McGehee S, et al. Mapping of human brown adipose tissue in lean and obese young men. Proceedings of the National Academy of Sciences. 2017 Aug 8;114(32):8649-54.

32. Berry DC, Stenesen D, Zeve D, Graff JM. The developmental origins of adipose tissue. Development. 2013 Oct;140(19):3939-49.

33. Seron-Ferre M, Reynolds H, Mendez NA, Mondaca M, Valenzuela F, Ebensperger R, et al. Impact of Maternal Melatonin Suppression on Amount and Functionality of Brown Adipose Tissue (BAT) in the Newborn Sheep. Frontiers in Endocrinology [Internet]. 2015 Jan 6 [cited $2020 \mathrm{Feb}$ 1];5. Available from: http://journal.frontiersin.org/ article/10.3389/fendo.2014.00232/abstract

34. Wang Q, Zhang M, Xu M, Gu W, Xi Y, Qi L, et al. Brown adipose tissue activation is inversely related to central obesity and metabolic parameters in adult human. PLoS ONE. 2015;10(4):e0123795. 
35. Orava J, Nuutila P, Lidell ME, Oikonen V, Noponen T, Viljanen T, et al. Different Metabolic Responses of Human Brown Adipose Tissue to Activation by Cold and Insulin. Cell Metabolism. 2011 Aug;14(2):272-9.

36. Ouellet V, Labbé SM, Blondin DP, Phoenix S, Guérin B, Haman F, et al. Brown adipose tissue oxidative metabolism contributes to energy expenditure during acute cold exposure in humans. Journal of Clinical Investigation. 2012 Feb 1;122(2):545-52.

37. Gesta S, Tseng Y-H, Kahn CR. Developmental Origin of Fat: Tracking Obesity to Its Source. Cell. 2007 Oct;131(2):242-56.

38. Billon N, Monteiro MC, Dani C. Developmental origin of adipocytes: new insights into a pending question. Biology of the Cell. 2008 Oct;100(10):563-75.

39. Ailhaud G, Grimaldi P, Négrel R. Cellular and Molecular Aspects of Adipose Tissue Development. Annual Review of Nutrition. 1992 Jul;12(1):207-33.

40. MacDougald OA, Lane MD. Transcriptional Regulation of Gene Expression During Adipocyte Differentiation. Annual Review of Biochemistry. 1995 Jun;64(1):345-73.

41. Wang P, Mariman E, Keijer J, Bouwman F, Noben J-P, Robben J, et al. Profiling of the secreted proteins during 3T3-L1 adipocyte differentiation leads to the identification of novel adipokines. Cellular and Molecular Life Sciences [Internet]. 2004 Sep [cited 2020 Jan 26];61(18). Available from: http://link.springer.com/10.1007/s00018-004-4256-z

42. Cartwright M, Tchkonia T, Kirkland J. Aging in adipocytes: Potential impact of inherent, depot-specific mechanisms. Experimental Gerontology. 2007 Jun;42(6):463-71.

43. Farmer SR. Molecular determinants of brown adipocyte formation and function. Genes \& Development. 2008 May 15;22(10):1269-75.

44. Nnodim JO, Lever JD. The pre- and postnatal development and ageing of interscapular brown adipose tissue in the rat. Anatomy and Embryology. 1985 Dec;173(2):215-23

45. Merklin RJ. Growth and distribution of human fetal brown fat. The Anatomical Record. 1974 Mar;178(3):637-45.

46. Richter HG, Torres-Farfan C, Garcia-Sesnich J, Abarzua-Catalan L, Henriquez MG, Alvarez-Felmer M, et al. Rhythmic Expression of Functional MT1 Melatonin Receptors in the Rat Adrenal Gland. Endocrinology. 2008 Mar;149(3):995-1003.

47. Mendez N, Abarzua-Catalan L, Vilches N, Galdames HA, Spichiger C, Richter HG, et al. Timed Maternal Melatonin Treatment Reverses Circadian Disruption of the Fetal Adrenal Clock Imposed by Exposure to Constant Light. Foulkes NS, editor. PLoS ONE. 2012 Aug 13;7(8):e42713.

48. van Mark A, Spallek M, Kessel R, Brinkmann E. Shift work and pathological conditions. J Occup Med Toxicol. 2006 Dec 11;1:25.

49. Erren TC, Reiter RJ. Defining chronodisruption. J Pineal Res. 2009 Apr;46(3):245-7.

50. Richter HG, Torres-Farfán C, Rojas-García PP, Campino C, Torrealba F, Serón-Ferré M. The circadian timing system: making sense of day/night gene expression. Biol Res. 2004;37(1):11-28.

51. Serón-Ferré M, Mendez N, Abarzua-Catalan L, Vilches N, Valenzuela FJ, Reynolds HE, et al. Circadian rhythms in the fetus. Molecular and Cellular Endocrinology. 2012 Feb;349(1):68-75.

52. Torres-Farfan C, Valenzuela FJ, Mondaca M, Valenzuela GJ, Krause B, Herrera EA, et al. Evidence of a role for melatonin in fetal sheep physiology: direct actions of melatonin on fetal cerebral artery, brown adipose tissue and adrenal gland: Functional melatonin receptor in fetal sheep tissues. The Journal of Physiology. 2008 Aug 15;586(16):4017-27.

53. Sun M, Feng W, Wang F, Li P, Li Z, Li M, et al. Meta-analysis on shift work and risks of specific obesity types. Obes Rev. 2018 Jan;19(1):28-40.

54. Liu Q, Shi J, Duan P, Liu B, Li T, Wang C, et al. Is shift work associated with a higher risk of overweight or obesity? A systematic review of observational studies with meta-analysis. Int J Epidemiol. 2018 01:47(6):1956-71.

55. Grundy A, Cotterchio M, Kirsh VA, Nadalin V, Lightfoot N, Kreiger N. Rotating shift work associated with obesity in men from northeastern Ontario. Health Promot Chronic Dis Prev Can. 2017 Aug;37(8):238-47.

56. Zvonic S, Ptitsyn AA, Conrad SA, Scott LK, Floyd ZE, Kilroy G, et al. Characterization of peripheral circadian clocks in adipose tissues. Diabetes. 2006 Apr;55(4):962-70.

57. Christou S, Wehrens SMT, Isherwood C, Möller-Levet CS, Wu H, Revell VL, et al. Circadian regulation in human white adipose tissue revealed by transcriptome and metabolic network analysis. Scientific Reports [Internet]. 2019 Dec [cited 2019 Apr 1];9(1). Available from: http:/ /www. nature.com/articles/s41598-019-39668-3

58. Kolbe I, Husse J, Salinas G, Lingner T, Astiz M, Oster H. The SCN Clock Governs Circadian Transcription Rhythms in Murine Epididymal White Adipose Tissue. Journal of Biological Rhythms. 2016 Dec;31(6):577-87.

59. Ptitsyn AA, Zvonic S, Conrad SA, Scott LK, Mynatt RL, Gimble JM. Circadian clocks are resounding in peripheral tissues. PLoS Comput Biol. 2006 Mar;2(3):e16.
60. Loboda A, Kraft WK, Fine B, Joseph J, Nebozhyn M, Zhang C, et al Diurnal variation of the human adipose transcriptome and the link to metabolic disease. BMC Med Genomics. 2009 Feb 9;2:7.

61. Stenvers DJ, Jongejan A, Atiqi S, Vreijling JP, Limonard EJ, Endert E, et al. Diurnal rhythms in the white adipose tissue transcriptome are disturbed in obese individuals with type 2 diabetes compared with lean control individuals. Diabetologia. 2019 Apr;62(4):704-16.

62. Gómez-Santos C, Gómez-Abellán P, Madrid JA, Hernández-Morante JJ, Lujan JA, Ordovas JM, et al. Circadian Rhythm of Clock Genes in Human Adipose Explants. Obesity. 2009 Aug;17(8):1481-5.

63. Stunkard AJ, Grace WJ, Wolff HG. The night-eating syndrome. The American Journal of Medicine. 1955 Jul;19(1):78-86.

64. Wade GN. Dietary obesity in golden hamsters: Reversibility and effects of sex and photoperiod. Physiology \& Behavior. 1983 Jan;30(1):131-7.

65. Bartness TJ, Wade GN. Photoperiodic Control of Body Weight and Energy Metabolism in Syrian Hamsters (Mesocricetus auratus): Role of Pineal Gland, Melatonin, Gonads, and Diet*. Endocrinology. 1984 Feb;114(2):492-8.

66. Wade GN, Bartness TJ. Effects of photoperiod and gonadectomy on food intake, body weight, and body composition in Siberian hamsters. American Journal of Physiology-Regulatory, Integrative and Comparative Physiology. 1984 Jan 1;246(1):R26-30.

67. Stunkard AJ, Allison KC, Lundgren JD, O'Reardon JP. A biobehavioural model of the night eating syndrome. Obesity Reviews. 2009 Nov;10:69-77.

68. Polonsky KS, Given BD, Van Cauter E. Twenty-four-hour profiles and pulsatile patterns of insulin secretion in normal and obese subjects. Journal of Clinical Investigation. 1988 Feb 1;81(2):442-8.

69. Van Cauter EV, Polonsky KS, Blackman JD, Roland D, Sturis J, Byrne $\mathrm{MM}$, et al. Abnormal temporal patterns of glucose tolerance in obesity: relationship to sleep-related growth hormone secretion and circadian cortisol rhythmicity. The Journal of Clinical Endocrinology \& Metabolism. 1994 Dec;79(6):1797-805.

70. Van Cauter E, Polonsky KS, Scheen AJ. Roles of circadian rhythmicity and sleep in human glucose regulation. Endocr Rev. 1997 Oct;18(5):716-38.

71. Van Cauter E, Blackman JD, Roland D, Spire JP, Refetoff S, Polonsky KS. Modulation of glucose regulation and insulin secretion by circadian rhythmicity and sleep. Journal of Clinical Investigation. 1991 Sep 1;88(3):934-42.

72. Lemberger'T, Desvergne B, Wahli W. PEROXISOME PROLIFERATORACTIVATED RECEPTORS: A Nuclear Receptor Signaling Pathway in Lipid Physiology. Annual Review of Cell and Developmental Biology. 1996 Nov;12(1):335-63

73. Brewer M, Lange D, Baler R, Anzulovich A. SREBP-1 as a Transcriptional Integrator of Circadian and Nutritional Cues in the Liver. Journal of Biological Rhythms. 2005 Jun;20(3):195-205.

74. Canaple L, Rambaud J, Dkhissi-Benyahya O, Rayet B, Tan NS, Michalik L, et al. Reciprocal Regulation of Brain and Muscle Arnt-Like Protein 1 and Peroxisome Proliferator-Activated Receptor $\alpha$ Defines a Novel Positive Feedback Loop in the Rodent Liver Circadian Clock. Molecular Endocrinology. 2006 Aug;20(8):1715-27.

75. Yin L, Wu N, Curtin JC, Qatanani M, Szwergold NR, Reid RA, et al. Rev-erb , a Heme Sensor That Coordinates Metabolic and Circadian Pathways. Science. 2007 Dec 14;318(5857):1786-9

76. Sinha MK, Ohannesian JP, Heiman ML, Kriauciunas A, Stephens TW, Magosin S, et al. Nocturnal rise of leptin in lean, obese, and non-insulindependent diabetes mellitus subjects. Journal of Clinical Investigation. 1996 Mar 1;97(5):1344-7.

77. Ahmad AM, Guzder R, Wallace AM, Thomas J, Fraser WD, Vora JP. Circadian and Ultradian Rhythm and Leptin Pulsatility in Adult GH Deficiency: Effects of GH Replacement. The Journal of Clinical Endocrinology \& Metabolism. 2001 Aug;86(8):3499-506.

78. Arasaradnam M, Morgan L, Wright J, Gama R. Diurnal variation in lipoprotein lipase activity. Annals of Clinical Biochemistry. 2002 Mar 1;39(2):136-9.

79. Kok SW, Meinders AE, Overeem S, Lammers GJ, Roelfsema F, Frölich $\mathrm{M}$, et al. Reduction of Plasma Leptin Levels and Loss of Its Circadian Rhythmicity in Hypocretin (Orexin)-Deficient Narcoleptic Humans. The Journal of Clinical Endocrinology \& Metabolism. 2002 Feb;87(2):805-9.

80. Gavrila A, Peng C-K, Chan JL, Mietus JE, Goldberger AL, Mantzoros CS. Diurnal and Ultradian Dynamics of Serum Adiponectin in Healthy Men: Comparison with Leptin, Circulating Soluble Leptin Receptor, and Cortisol Patterns. The Journal of Clinical Endocrinology \& Metabolism. 2003 Jun;88(6):2838-43.

81. Yildiz BO, Suchard MA, Wong M-L, McCann SM, Licinio J. Alterations in the dynamics of circulating ghrelin, adiponectin, and leptin in human obesity. Proceedings of the National Academy of Sciences. $2004 \mathrm{Jul}$ 13;101(28):10434-9.

82. Bray MS, Young ME. The role of cell-specific circadian clocks in metabolism and disease. Obesity Reviews. 2009 Nov;10:6-13. 
83. Cermakian N, Boivin DB. The regulation of central and peripheral circadian clocks in humans. Obesity Reviews. 2009 Nov;10:25-36.

84. Johnston JD, Frost G, Otway DT. Adipose tissue, adipocytes and the circadian timing system. Obesity Reviews. 2009 Nov;10:52-60.

85. Langley-Evans SC, McMullen S. Developmental Origins of Adult Disease. Medical Principles and Practice. 2010;19(2):87-98.

86. Entringer S, Buss C, Wadhwa PD. Prenatal stress and developmental programming of human health and disease risk: concepts and integration of empirical findings: Current Opinion in Endocrinology, Diabetes and Obesity. 2010 Dec;17(6):507-16.

87. Thompson BL, Levitt P, Stanwood GD. Prenatal exposure to drugs: effects on brain development and implications for policy and education. Nature Reviews Neuroscience. 2009 Apr;10(4):303-12.

88. Barker D. INFANT MORTALITY, CHILDHOOD NUTRITION, AND ISCHAEMIC HEART DISEASE IN ENGLAND AND WALES. The Lancet. 1986 May;327(8489):1077-81.

89. Ravelli AC, van der Meulen JH, Osmond C, Barker DJ, Bleker OP. Obesity at the age of $50 \mathrm{y}$ in men and women exposed to famine prenatally. The American Journal of Clinical Nutrition. 1999 Nov 1;70(5):811-6.

90. Ford SP, Hess BW, Schwope MM, Nijland MJ, Gilbert JS, Vonnahme $\mathrm{KA}$, et al. Maternal undernutrition during early to mid-gestation in the ewe results in altered growth, adiposity, and glucose tolerance in male offspring1. Journal of Animal Science. 2007 May 1;85(5):1285-94.

91. Pinheiro AR, Salvucci IDM, Aguila MB, Mandarim-de-Lacerda CA. Protein restriction during gestation and/or lactation causes adverse transgenerational effects on biometry and glucose metabolism in F1 and F2 progenies of rats. Clinical Science. 2008 Mar 1;114(5):381-92.

92. Bouanane S, Benkalfat NB, Baba Ahmed F-Z, Merzouk H, Mokhtari NS, Merzouk S-A, et al. Time course of changes in serum oxidant/antioxidant status in overfed obese rats and their offspring. Clinical Science. $2009 \mathrm{Apr}$ 1;116(8):669-80.

93. Crispi F, Miranda J, Gratacós E. Long-term cardiovascular consequences of fetal growth restriction: biology, clinical implications, and opportunities for prevention of adult disease. American Journal of Obstetrics and Gynecology. 2018 Feb;218(2):S869-79.

94. Martin RJ, Hausman GJ, Hausman DB. Regulation of Adipose Cell Development In Utero. Experimental Biology and Medicine. 1998 Dec 1;219(3):200-10.

95. Barker M, Robinson S, Osmond C, Barker DJP. Birth weight and body fat distribution in adolescent girls. Archives of Disease in Childhood. 1997 Nov 1;77(5):381-3.

96. Vickers MH, Breier BH, Cutfield WS, Hofman PL, Gluckman PD. Fetal origins of hyperphagia, obesity, and hypertension and postnatal amplification by hypercaloric nutrition. American Journal of PhysiologyEndocrinology and Metabolism. 2000 Jul 1;279(1):E83-7.

97. Perseghin G, Ghosh S, Gerow K, Shulman GI. Metabolic defects in lean nondiabetic offspring of NIDDM parents: a cross-sectional study. Diabetes. 1997 Jun 1;46(6):1001-9.

98. Valsamakis G, Kanaka-Gantenbein C, Malamitsi-Puchner A, Mastorakos G. Causes of Intrauterine Growth Restriction and the Postnatal Development of the Metabolic Syndrome. Annals of the New York Academy of Sciences. 2006 Dec 1;1092(1):138-47.
99. Barker DJP, Hales CN, Fall CHD, Osmond C, Phipps K, Clark PMS Type 2 (non-insulin-dependent) diabetes mellitus, hypertension and hyperlipidaemia (syndrome $\mathrm{X}$ ): relation to reduced fetal growth. Diabetologia. 1993 Jan;36(1):62-7.

100. Andrew R, Phillips DIW, Walker BR. Obesity and Gender Influence Cortisol Secretion and Metabolism in Man. The Journal of Clinical Endocrinology \& Metabolism. 1998 May;83(5):1806-1806.

101. Ong KKL. Association between postnatal catch-up growth and obesity in childhood: prospective cohort study. BMJ. 2000 Apr 8;320(7240):967-71.

102. the EURO-BLCS Study Group, Rogers I. The influence of birthweight and intrauterine environment on adiposity and fat distribution in later life. International Journal of Obesity. 2003 Jul;27(7):755-77.

103. Yajnik C. Interactions of perturbations in intrauterine growth and growth during childhood on the risk of adult-onset disease. Proceedings of the Nutrition Society. 2000 May;59(2):257-65.

104. Stocker CJ, Arch JRS, Cawthorne MA. Fetal origins of insulin resistance and obesity. Proceedings of the Nutrition Society. 2005 Mav;64(2):143-51.

105. Jaquet D, Deghmoun S, Chevenne D, Collin D, Czernichow P, LévyMarchal C. Dynamic change in adiposity from fetal to postnatal life is involved in the metabolic syndrome associated with reduced fetal growth. Diabetologia. 2005 May;48(5):849-55.

106. Silveira VMF da, Horta BL. Peso ao nascer e síndrome metabólica em adultos: meta-análise. Revista de Saúde Pública. 2008 Feb;42(1):10-8.

107. Motta-Teixeira LC, Machado-Nils AV, Battagello DS, Diniz GB, Andrade-Silva J, Silva S, et al. The absence of maternal pineal melatonin rhythm during pregnancy and lactation impairs offspring physical growth, neurodevelopment, and behavior. Hormones and Behavior. 2018 Sep;105:146-56.

108. Serón-Ferré M, Forcelledo ML, Torres-Farfan C, Valenzuela FJ, Rojas A, Vergara $\mathrm{M}$, et al. Impact of chronodisruption during primate pregnancy on the maternal and newborn temperature rhythms. PLoS ONE. 2013;8(2):e57710.

109. Nehme PA, Amaral F, Lowden A, Skene DJ, Cipolla-Neto J, Moreno CRC. Reduced melatonin synthesis in pregnant night workers: Metabolic implications for offspring. Medical Hypotheses. 2019 Nov;132:109353.

110. Cai C, Vandermeer B, Khurana R, Nerenberg K, Featherstone R, Sebastianski M, et al. The impact of occupational shift work and working hours during pregnancy on health outcomes: a systematic review and meta-analysis. American Journal of Obstetrics and Gynecology. 2019 Dec;221(6):563-76.

111. Bonzini M, Palmer K, Coggon D, Carugno M, Cromi A, Ferrario M. Shift work and pregnancy outcomes: a systematic review with metaanalysis of currently available epidemiological studies: Shift work and pregnancy outcomes. BJOG: An International Journal of Obstetrics \& Gynaecology. 2011 Nov;118(12):1429-37.

112. Specht IO, Hammer PEC, Flachs EM, Begtrup LM, Larsen AD, Hougaard KS, et al. Night work during pregnancy and preterm birth-A large register-based cohort study. Szecsi PB, editor. PLOS ONE. 2019 Apr 18;14(4):e0215748. 


\section{Annual variation of daily activity patterns and its dependence on photoperiod: a quick overview}

Giovane Carreira Improta

Laboratorio Binacional Argentina-Brasil de Cronobiologia, Departamento de Fisiologia, Instituto de Biociências, Universidade de São Paulo, São Paulo, Brazil.

\begin{abstract}
Earth's environmental cycles, such as daily light/dark (LD) and annual photoperiodic variation (ratio between day/night duration), can synchronize endogenous rhythms with periods of $24 \mathrm{~h}$ and 1 year, respectively. Here some evidence pointing to an interconnectivity between the synchronization of circadian and annual rhythms will be briefly presented, most notably presenting the annual modulation of the activity/rest daily rhythms in field and lab conditions.
\end{abstract}

Keywords: Annual Rhythms; Activity Rhythm; Seasonality; Photoperiodism; Mammal; Infradian

Corresponding author:

Giovane Carreira Improta

E-mail: giovane.improta@usp.br

DOI: $10.5935 / 1984-0063.20200015$ 
Life on Earth is subject to the influence of several regular natural cycles, most notably astronomical and geophysical ones. Synchronization of internally generated rhythms by environmental cues has conferred a great selective advantage on living organisms in our cyclic environment.

Among the endogenous biological rhythms, the best known are the circadian thythms, called in this way (from the latin circa+diem) because they have a period of "about a day" (period of the Earth's day $=24 \mathrm{~h}$ ). The persistence of rhythms, with a period close to $24 \mathrm{~h}$ under constant darkness (DD) was the central evidence that triggered the study of these rhythms. The structures responsible for the generation and regulation of endogenous circadian rhythms are called circadian oscillators ${ }^{1}$ and, in mammals, they are anatomically located in the suprachiasmatic nuclei $(\mathrm{SCN})$ of the hypothalamus ${ }^{2}$. Circadian oscillators are synchronizable by environmental cycles called Zeitgebers. Zeitgebers' signals are able to induce adjustments in the dynamics of the oscillator thus attuning their timing in a process called entrainment.

Among the many rhythms that organisms can express in a circadian manner, the activity/rest rhythm is especially interesting because the temporal niche, associated with the daily light/dark (LD) cycle, allocates a set of behaviors in time thus optimizing energy expenditure and, essentially, the chance of individual survival and continuity of the lineage. Being active at a particular phase of day or night has enormous ecological significance, and it is through this timing system that organisms can anticipate various cyclical environmental challenges ${ }^{3,4}$.

Under isolated conditions, some properties of selfsustained endogenous rhythms can be better studied, one of the most important being the period of oscillation $(\tau)$ in the absence of Zeitgebers. Biological rhythms with periods other than $24 \mathrm{~h}$ are known as ultradian when their period is shorter than a day's duration $(\tau<24 \mathrm{~h})$, and infradian in the case of rhythms with a period longer than one day $(\tau>24 \mathrm{~h})$, as is the case with the circalunar and circannual rhythms. The latter display $\tau$ close to a year and is often synchronized by environmental cycles with periods of 1 year.

Despite the great reliability of the daily natural light/ dark cycle generated by the Earth's rotational motion around its axis, the ratio between the duration of the two phases of this cycle (photophase - light phase, i.e. "day" and scotophase dark phase, i.e. "night") is not the same all year round. The ratio between photophase and scotophase of the daily cycle is called photoperiod.

The annual variation in the photoperiod is caused by a tilt of about $23.5^{\circ}$ - also known as obliquity - in Earth's rotation axis $($ period $=24 \mathrm{~h})$ from the plane in which it orbits the Sun (period $=1$ year). Given this tilt, the planet receives uneven solar radiation and as the planet keeps orbiting the Sun, the side that is tilted towards the sun changes regularly: At the solstices, the hemisphere that is tilted towards the Sun enters summer and presents a longer photophase and shorter scotophase ("long days"), and the opposite hemisphere enters winter, with shorter photophase and longer scotophase ("short days").
This is followed by an equinox in which photophase and scotophase have a similar duration (fall and spring) and then another solstice switches the hemisphere tilted towards the Sun.

Several annual biological rhythms have been studied and these events are related to adopting a temporal strategy to overcome an environmental challenge imposed by seasons. These strategies are usually divided into 3 categories 5 :

(i) Dormancy in which the organism physiologically regulates a state of metabolic depression, as in the case of estivation (quite common avoiding high temperatures and desiccation in summer, especially in equatorial and tropical areas), hibernation (during winter in temperate and polar zones) and diapause;

(ii) Seasonal polyphenism refers to the programmed change in morphological phenotype to a more suitable form for a given season, as in the case of changing the color and thickness of the layer of fur or feathers;

(iii) Migration, which involves spatial dislocation. Instead of adjusting physiology and morphology while remaining in their habitat, populations move annually to specific areas with proper climatic conditions and improved food availability.

Whatever the strategy adopted to overcome seasonal environmental challenges, radical changes in metabolism, behavior and a considerable amount of energy are required, therefore, a series of preparatory actions need to be taken in anticipation of the occurrence of these phenomena. Additionally, reproduction is usually also associated with a specific phase of the year, thus increasing the need for efficient timing between all these events ${ }^{6}$.

Just as the daily LD cycle synchronizes most of circadian rhythms, the annual variation of the photoperiod is currently viewed as the most important synchronizer for several annual rhythms $s^{7}$. The photoperiodic cue is involved in preparations that trigger some annual phenomena, and many authors call it a "proximate factor" - i.e., the cue is transduced and initiates preparations for changes (e.g. pre-migratory hyperphagia), but not necessarily triggers the strategy (e.g. migration) by itself.

The role of photoperiod as an annual Zeitgeber found experimental evidence when Gwinner ${ }^{8}$ demonstrated synchronized circannual rhythms synchronized to "annual" photoperiods with periods other than 12 months. To some extent, this is equivalent to the synchronization of individuals to LD cycles with periods other than 24 hours in the circadian scale.

Back to the daily scale, some properties of an animal's daily activity/rest rhythm are closely associated with a specific phase of the LD cycle, for example, the activity onset. Wildlife monitoring studies in bears have shown, for instance, that the onset of daily activity already occurs before being exposed to dawn light, indicating that before daily light exposure, there is physiological and behavioral anticipation conferred by internal rhythms synchronized by environmental cycles ${ }^{9}$. 
Importantly the activity onset followed the changes in photoperiod all year long, delaying or advancing its timing accordingly, to later or earlier times of dawn in winter and summer, respectively. Studies on captive brown bears ${ }^{10}$ exposed to controlled photoperiod changes showed that daily activity is highly modifiable by food availability and photoperiod in a "stereotypical seasonal fashion", especially at some phases of the year.

In nature, as shown by Daan and Aschoff ${ }^{11}$ with daily activity monitoring on captive birds and rodents under natural photoperiodic changes, there is variation in the onset phase, and also in activity duration $(\alpha)$ of the daily activity/rest cycle of various species throughout the year. The annual activity pattern and its degree of change are strongly associated with the annual variation of the photoperiod (being more marked the greater the latitude, given the obliquity).

In parallel to the records of animals under the natural annual variation of the photoperiod, laboratory studies are conducted focusing on activity patterns under different photoperiods. In captivity, it is possible to manipulate the photoperiod simply by changing the times of lights on and off of artificial light/dark cycles in the confined environment while other variables are kept at desired levels. Despite the loss of the natural environment elements and the reduced ecological value of measurements, important aspects of rhythms are elucidated when the system is investigated in controlled environments.

By setting lights on and off times, important aspects of the activity pattern become quantifiable as in the study conducted by DeCoursey and collaborators ${ }^{12}$ in which they found that despite changes in $\alpha$ under different photoperiods, the phase relationship $(\psi)$ - i.e. the time difference between a phase of the LD cycle (for instance, lights-off) and of the activity onset - remains stable, that is, the time of activity onset in a nocturnal animal, for instance, changes throughout the year but the interval between dusk and activity onset remains the same. The stability of the phase relationship was so pronounced that it was even proposed to use it to distinguish between nocturnal and diurnal species.

However, it was found that $\psi$ remained fixed only within a specific range of photoperiods (those photoperiods close to $\mathrm{LD}$ 12:12). Out of this limit, unusual phase responses appeared and it became difficult to model exactly how daily activity patterns change in response to different photoperiods throughout all photoperiod ranges $^{13}$. In addition to the modulation of $\psi$, another important aspect of the annual modulation of daily activity rhythm by photoperiod is its effects on the daily duration of activity $(\alpha)$ with longer activity during summer for a diurnal animal, for instance. To understand the mechanism in which the duration of activity $(\alpha)$ varied throughout the year, it was proposed that the circadian oscillator is composed of two coupled oscillators (i.e., E - evening and M - morning). In 1976, Pittendrigh and Daan ${ }^{14}$, proposed and indicated that this model explained more sparingly "seasonal patterns" such as those found by Daan \& Aschoff's work in $1975^{11}$. It is now accepted that there is an emerging annual modulation of the daily rhythm generated by two coupled oscillators. The annual modulation of daily activity rhythm can be a useful measure to further understand this dynamics and to test this model ${ }^{15}$.
Photoperiod is capable of modifying the function or even the internal arrangement of some structures responsible for daily rhythms regulation. In birds, it has been found that the pineal gland (responsible for the circadian rhythms in birds) changes the pattern in which it releases melatonin in response to photoperiodic information, even when the gland is surgically removed and maintained in vitro, meaning that this structure is able to keep a record of the photoperiodic history by itself ${ }^{16}$. The pineal gland acts as a regulatory organ in vertebrate animals by secreting melatonin, a neurohormone with a conspicuous annual variation of daily release pattern, resembling in some manner the annual modulation of the daily activity patterns.

In mammals, astounding electrophysiological evidence show the persistence of distinctive photoperiodic induced (while, in vivo) neuronal activity patterns on hamster SCN slices (this time, in vitro) ${ }^{17}$.

There is still much to be discovered, given the complexity and vastness of factors involved in the timing of annual rhythms, especially on how the photoperiodic cues are translated into modulation of daily rhythms throughout the year.

While photoperiods with natural-like photophase and scotophase ratios reveal the stability of $\psi$, with major and important ecological implications, experiments with extreme "short days" and "long days" photoperiods help us unveil the oscillator dynamics involved in the processing and expression of annual rhythmic patterns.

New investigations could benefit from studies of natural, extreme photic environments. In our group, we have studied the tuco-tuco (Ctenomys aff. knighti), a subterranean rodent that exposes itself to light in random times throughout the day ${ }^{18}$. They have shown evidence for seasonal difference in their daily activity patterns: tuco-tucos caught in winter and summer had significantly different $\alpha$ on running wheel activity under captive conditions ${ }^{19} \mathrm{It}$ is important to point out that exhibiting an annual rhythm does not necessarily mean that the organism is, undoubtedly photoperiodic. Further investigations are taking place to determine if the nature of these yearly changes are due to other seasonal cues in the subterranean, or if the photoperiod cue is sufficient to induce these modulations on activity patterns, as appears to be the case in most of the other mammals across the phylogeny.

Another interesting aspect of investigating a subterranean animal relies on modeling how much light exposure is capable of giving enough information for annual photoperiodic processing in extreme photic environments such as burrows and caves.

\section{ACKNOWLEDGEMENTS}

This work was only possible because of the inestimable discussions with Gisele Oda, Verónica Valentinuzzi, Horacio de la Iglesia, Danilo Flôres, Patricia Tachinardi, Milene Jannetti, Jefferson Silva, and Tamiris Yassumoto. I would like to especially thank Gisele Oda for the thoughtful proofreading and overall writing assistance, Johanna Barros for exceptional technical assistance on current experiments, Patrícia Komeih Romero for providing crucial language help, and the XV Latin American Symposium on Chronobiology (LASC) organizing committee. 


\section{REFERENCES}

1. Dunlap C, Loros JJ, DeCoursey PJ. Chronobiology: biological timekeeping, editors. Sinauer Associates, 2004.

2. Moore-Ede MC, Czeisler CA, Richardson GS. Circadian timekeeping in health and disease: basic properties of circadian pacemakers. New England Journal of Medicine. 1938; 309.8: 469-476.

3. Halle S, Stenseth NC, editors. Activity patterns in small mammals. Berlin, Heidelberd:Springler-Verlag; 2000. pp. 217-243.

4. Tomotani BM, Flôores DEFL, Tachinardi P, Paliza JD, Oda, GA, Valentinuzzi VS. Field and laboratory studies provide insights into the meaning of day-time activity in a subterranean rodent (Ctenomys aff. knighti), the tuco-tuco. PLoS One. 2012; 7(5), e37918.

5. Tauber MJ, Tauber CA, Masaki S. Adaptations to hazardous seasonal conditions: dormancy, migration, and polyphenism. In: Hufftaker CB, Rabb RL, editors. Ecological entomology. California: Raleigh; 1984.

6. Kenagy GJ, Bartholomew GA. Seasonal Reproductive Patterns in Five Coexisting California Desert Rodent Species: Ecological Archives M055002. Ecological Monographs. 1985; 55(4): 371-397.

7. Gwinner E, Helm B. Circannual and circadian contributions to the timing of avian migration. In: Avian migration. Springer, Berlin, Heidelberg; 2003. pp. $81-95$

8. Gwinner E. Circannual systems. In: Biological rhythms. Springer, Boston, MA; 1981, pp. 391-410

9. Fortin JK, Schwartz CC, Gunther KA, Teisberg JE, Haroldson MA, Evans MA, et al. Dietary adjustability of grizzly bears and American black bears in Yellowstone National Park. The Journal of wildlife management. 2013; 77(2):270-281.

10. Ware JV, Nelson OL, Robbins CT, Jansen HT. Temporal organization of activity in the brown bear (Ursus arctos): roles of circadian rhythms, light, and food entrainment. American Journal of Physiology-Regulatory, Integrative and Comparative Physiology. 2012; 303(9):R890-R902.

11. Daan S, Aschoff J. Circadian rhythms of locomotor activity in captive birds and mammals: their variations with season and latitude. Oecologia. 1975; 18(4):269-316.

12. DeCoursey PJ. LD ratios and the entrainment of circadian activity in a nocturnal and a diurnal rodent. Journal of comparative physiology. 1972; 78(3):221-235.

13. Elliott JA. Circadian rhythms and photoperiodic time measurement in mammals. In: Federation proceedings. 1976; Vol. 35, No. 12, pp. 2339-2346.

14. Pittendrigh CS, Daan S. A functional analysis of circadian pacemakers in nocturnal rodents. Journal of comparative physiology. 1976; 106(3):223-252.

15. Flôres DEFL, Oda GA. Quantitative study of dual circadian oscillator models under different skeleton photoperiods. Journal of Biological Rhythms. Forthcoming 2020.

16. Brandstätter R, Kumar V, Abraham U, Gwinner E. Photoperiodic information acquired and stored in vivo is retained in vitro by a circadian oscillator, the avian pineal gland. Proceedings of the National Academy of Sciences. 2000; 97(22):12324-12328.

17. Mrugala M, Zlomanczuk P, Jagota A, Schwartz WJ. Rhythmic multiunit neural activity in slices of hamster suprachiasmatic nucleus reflect prior photoperiod. American Journal of Physiology-Regulatory, Integrative and Comparative Physiology. 2000; 278(4):R987-R994.

18. Flôres DEFL, Jannetti MG, Valentinuzzi VS, Oda GA. Entrainment of circadian rhythms to irregular light/dark cycles: a subterranean perspective. Scientific reports. 2016; 6, 34264.

19. Improta GC, Oda GA, Valentinuzzi VS. The dependence of daily patterns of activity on photoperiod in a subterranean rodent (Ctenomys aff. knighti): Effects on synchronization and aftereffects of entrainment [abstract]. XV Latin American Symposium on Chronobiology. 2019. 


\section{Different latitude influences the habitat and reproductive patterns of Pinnipeds}

Brenda Morales-Pérez ${ }^{1}$

María del Carmen Miñana-Solís ${ }^{2 *}$

${ }^{1}$ Instituto Politécnico Nacional, Departamento de Pesquerías y Biología Marina, La Paz, Baja California Sur, México.

${ }^{2}$ Universidad Nacional Autónoma de México, Facultad de Ciencias, Ciudad de México, México.

\begin{abstract}
Pinnipeds are marine mammals that have a fusiform body with the anterior and posterior finshaped members that facilitate its movement in the water. They are distributed in all the seas of the world, mainly in polar, subpolar and temperate waters. Apparently diverse environmental factors have influenced their evolution of the shape and the mating system, as an adaptative strategy to ensure the optimal temporal use of niches in the environment. Reproductive patterns are specific for each species, the latitude in which they live determines the mating system, reproductive cycle, and nursing strategies. In high latitudes, sexual dimorphism and polygyny are weak, and the birthing season is short. While in low latitudes, sexual dimorphism sexual is evident, polygyny is from moderate to extreme, and the birthing season is throughout the year. So that photoperiod is the main stimulus that shapes reproductive cycles, since it influences temperature and food availability, and the physiology of the organisms.
\end{abstract}

Keywords: Geographical Latitude; Pinnipeds; Reproduction
Corresponding author:

María del Carmen Miñana-Solís

E-mail:mcms@ciencias.unam.mx 


\section{CONTENT}

Pinnipeds are marine mammals of the Carnivorous Order. They have fusiform body with anterior and posterior fin-shaped members that facilitates its movement in water. They include three families: Phocidae, true seals without visible ears; Otariidae, fur seals and sea lions, with visible ears; and Odobenidae, which encomprise walruses (Fig. 1)2.
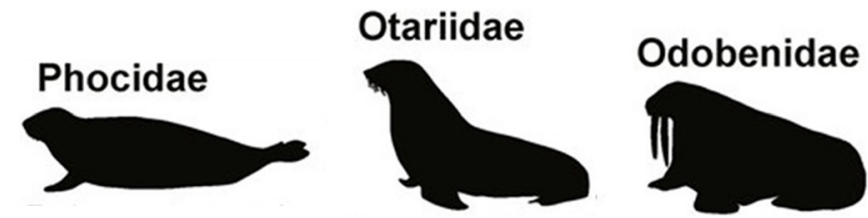

Figure 1. Schematic representation of the body shape of each group of pinnipeds Body characteristics facilitate locomotion in the environment in which they live. Modified from ${ }^{18}$.

These groups of organisms are distributed in all the seas of the world, mainly in polar, subpolar and temperate waters. Walruses present strictly Arctic distribution. The otariids form colonies in temperate latitudes ${ }^{3}$. There are two species of this group that are distributed near Ecuator, in the Galapagos Islands: The Galapagos fur seal (Arctophoca galapagoensis) and The Galapagos sea lion (Zallophus wollebaeki) ${ }^{4}$. The phocids are distributed mainly in middle and polar latitudes ${ }^{5}$, although there are two species with tropical distribution The Mediterranean monk seal (Monacbus monachus) and the Hawaiian monk seal (Monacbus schaninslandi).

Evidence shows that diverse environmental factors have influenced the evolution of the shape and the mating system of the pinnipeds ${ }^{3}$, as an adaptative strategy to ensure the optimal temporal use of niches in the environment ${ }^{7}$. None of the Antarctic colonies of pinnipeds are as dense as those colonies of species with land reproduction ${ }^{8}$. However, there are three exceptions, phocids species with behavior, and sexual dimorphism similar to otariids: the elephant seals (Mirounga angustirostris, Mirounga leonina) and the gray seal (Halichoerus grypus), which carry out their reproductive activities on land'.

Most pinnipeds have seasonal reproduction associated with food availability and environmental conditions (10); thus, optimizing the offspring survival and the reproductive synchrony. On top of that, the female receptivity to the male when they are able to copulate, maximizes the reproductive success of the individuals ${ }^{10}$.

\section{Mating systems and sexual dimorphism diversity}

Reproductive patterns are specific for each species, the latitude in which they live determines the mating system, reproductive cycle and nursing strategies ${ }^{11,12}$.

The species with preference to reproduce on land such as otariids and walruses, present polygyny from moderate (6-15 females) to extreme (16-100 females) ${ }^{9}$; due to the limited extension of the breeding $\operatorname{site}^{13}$. Terrestrial breeders show the most extreme polygyny due to the limited extent and predictable conditions of the breeding territory. The intrasexual competence of males is intense and as a consequence they have sexual dimorphism ${ }^{3}$ characterized by the bigger body size in males with respect to females ${ }^{4}$.
The degree of sexual dimorphism is related to the harem size ${ }^{14}$. Phocids tend to reproduce in water or ice floes (sites less stable than land), are monogamous or have weak polygyny (2-5 females) $)^{9}$. Aquatic breeders by contrast are predicted to show lower levels of polygyny due to the difficulties involved in defending either females or resources, as well as their weak or absent sexual dimorphism, because competition between males is not intense $e^{3}$.

All pinnipeds have embryonic diapause, or delayed blastocystimplantation, which consists in maintaining embryonic development in a latency state ${ }^{4,11}$. This characteristic is shared with the carnivorous species of the Ursidae and Mustelidae families. The pinnipeds are probably related to the ancestors of these groups and the embryonic diapause is an inheritance of the ancestral carnivorous line ${ }^{11}$. Delayed implantation ensures the birth of the pups occurs when the environmental conditions are optimal for their survival ${ }^{11}$.

The duration of embryonic diapause is specific for each species; this usually last 4 months ${ }^{10}$ and is followed by an active gestation that takes about 8 months in most species ${ }^{11}$. The mating season occurs a few days after birth. These activities are energetically expensive, so their temporal proximity during breeding season reduce the energy expenditure ${ }^{11}$. Females and males form aggregations during breeding season. Females gave birth only one offspring and are the only ones that which provide nutritional resources to their offspring, so their survival depends solely on the mother ${ }^{2,4}$.

Although pinnipeds are characterized by spending most of their time feeding at sea, their require a surface of land or ice on which to give birth ${ }^{2,11}$. In this way, nursing and food search are spatially and temporarily separated ${ }^{2}$.

In low latitudes, where the seasonal changes are less pronounced and predictable, the birth season is prolonged ${ }^{11,12}$. On the other hand, in high latitudes, seasonal changes are more predictable and the environmental conditions are more challenging. These characteristics promote the acquisition and reinforcement of seasonality in reproductive cycles ${ }^{6,14}$. In consequence, the nursing is short and migrations occur during the summer (which is brief) and the females must attend their offspring while the conditions are favorable ${ }^{16}$.

The duration of maternal care also is specific for each species: from a few days or weeks, for phocids, between 8 and 12 months for otariids, and 3 or more years for walruses. In function of this differences, three types of maternal strategies have been developed: fasting, feeding cycle or aquatic nursing ${ }^{2}$. Fasting is present in most phocids: females fast during lactation (4-50 days $)^{2,17}$. Its milk contains high fat concentrations, mainly in those species with short lactation periods ${ }^{2}$. In feeding cycle, females fast for 5-11 days after parturition, while they attend the pups and alternate land stays for nursing with trips to the sea to feed. The lactation takes 4 months to 3 years, this strategy is characteristic of otariids. Aquatic nursing is exclusive to walruses, the pups never separate from their mothers and nursing in water, where the mother is feeding, and in land or ice for 2 to 3 years $^{2,4,18}$. 
Several differences in the reproductive cycle have been shown depending on the latitude. Modifications in the environment that occur throughout the year, such ice coverage, or sites with land, productivity, light conditions, abundance and distribution of prey, are more evident in high latitudes ${ }^{5}$. In low latitudes the food availability, breeding sites and climatic conditions are optimal for reproduction throughout the year ${ }^{6}$. This has an effect in animal behavior, specially, in the amount of time they invest in the search for food and reproduction ${ }^{5}$.

Regarding phocid and otariid maternal behavior, the amount of time the offspring spends with the mother increases as latitude decreases ${ }^{6}$. In such a way, that nursing in high latitudes is intense and brief ${ }^{12}$.

The Crabeater seal (Lobodon carcinophagus), habits ice packages surrounding Antarctic. Nursing takes 17 days, 4 days later, estrous, ovulation and mating occurs ${ }^{8}$. In this species, like most of phocids, births take place in the ice package, and mating is usually in the water. The instability of the site prevents the establishment of polygynic groups, as a result, this species is monogamous ${ }^{19,20}$; in reproductive season form triads with a male, a female and pup, both sexes are similar on size $\mathrm{e}^{20}$.

The Antarctic fur seal (Arctocephalus gazella) is an otariid, with marked sexual dimorphism, males are 3-6 times bigger and heavier than females, they present polygyny with 10 females harems, and inhabit $54^{\circ} \mathrm{S}$ of latitude. The reproductive season, covers from October to December, when the males leave the site and the females nursing their pups for 4 months, in which feed trips from 3-6 days, are inserted ${ }^{16}$.

The Weddell seal (Leptonychotes weddellii), presents weak sexual dimorphism in body size, some authors reports that females are slightly larger than males ${ }^{21}$, moderate polygyny, as a result of the environment, because inhabits ice packages in Antarctic, nursing is prolonged, since the females make feeding trips during lactation ${ }^{13}$. Due the absence of terrestrial predators, predation risk is minimal, and breeding is concentrated around tidal cracks in the fast ice ${ }^{13}$

The Mediterranean monk seal (Monachus monachus) and the Hawaiian monk seal (Monachus schauinslandi) are the southernmost species of phocids, live in latitudes close to $20^{\circ} \mathrm{N}$, both have low seasonality in births and lactation. They reproduce throughout the year and nursing takes almost 4 months ${ }^{6}$. The Hawaiian monk seal are no sexually dimorphic ${ }^{22}$. While Mediterranean monk seal exhibits sexual dimorphism in pelage color (Fig. 2), copulation is in the water and the males defend aquatic territories like the Otariidae ${ }^{23}$.

The otariid California sea lion (Zalopbus californianus), inhabits along the Pacific Coast of North America and Península de Baja California, Mexico, throughout the Gulf of California (Fig. 2). ${ }^{24}$ Exhibits polygynyc mating system, notorious sexual dirmorphism and seasonal reproduction takes three months each year ${ }^{25}$.

The Galapagos fur seal (Arctocephalus galapagoensis) inhabits equatorial region, is polygynous and has sexual dimorphism. Their reproductive season covers approximately 5 months, which makes it difficult for males to monopolize females $^{26}$. Nursing in this species can take 3 years $^{12}$.

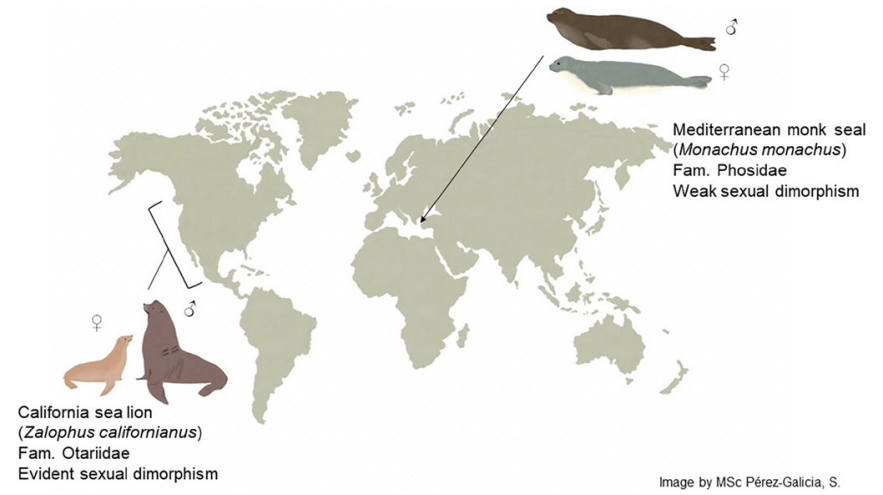

Figure 2. Schematic representation about sexual dimorphism and distribution of California sea lion and Mediterranean monk seal.

The walrus (Odobenus rosmarus) shows sexual dimorphism with polygyny for the defense of females?. Despite its circumpolar distribution, in the Arctic Atlantic from Canada to the Kara Sea in Russia, it does not present annual reproduction. Its reproductive cycle requires 2 years, embryonic diapause takes four months and the pups are weaned between 2 and 3 years ${ }^{27}$.

\section{Endocrine systems in the reproduction of Pinnipeds}

Reproduction is determined by seasonal neuroendocrine cycles that have evolved in temperate and polar latitudes ${ }^{7}$. Data on reproductive endocrinology in pinnipeds are limited due to difficulties to obtain serial samples from individuals ${ }^{11}$.

It is known that there is an increase in estrogen levels associated with the end of the embryonic diapause in northern fur seal (Callorbinus ursinus) ${ }^{10}$. However, it has been reported in the same species that is not possible to interrupt embryonic diapause when treating females with ovarian steroids, suggesting that changes in the levels of these hormones are not the cause, and it is a characteristic of implantation ${ }^{11}$. The increase in estrogen levels at the end of the embryonic diapause also has been reported in California sea lion (Zalophus californianus), without differences between pregnant and non-pregnant females ${ }^{28}$.

Another hormone involved in reproduction is the melatonin. Mammals with seasonal reproduction show changes in nocturnal secretion of melatonin that regulate gene expression in the pars tuberalis of the pituitary, so it has been proposed that this structure is involved in seasonal reproduction, because it has melatonin receptors $\mathrm{MT}^{29}$. The pars tuberalis represents the main circannual timer. It is composed of molecular machinery that is driven by external stimuli such as the photoperiod. Photoperiodic signals are perceived by retinal photoreceptors in mammals. To encode this information, mammals use the melatonin secreted for the pineal gland as a signal. This controls the molecular machinery and exit routs of pars tuberalis ${ }^{29}$.

Specifically, southern elephant seal (Mirounga leonina) adult males shows seasonal variation in plasma concentration of melatonin in response to photoperiod of circumpolar regions it inhabits $^{30}$. In Weddell seal (Leptonychotes weddellii) no diurnal variation in the concentrations of this hormone has been detected during the summer, when they are exposed to 24 hours of light $t^{11}$. 
Due to the synthesis of this hormone is inhibited by light ${ }^{30}$, the role of melatonin in reproduction of other mammals has also been demonstrated experimentally. In hamsters, which reproduction occurs during large photoperiods (more than $12 \mathrm{~h}$ of light per day), exogenous melatonin administrated to pinealectomized animals in a given phase of the circannual cycle synchronizes the endogen rhythm of reproduction ${ }^{15}$.

Progesterone has been reported to be a characteristic hormone of pregnancy. Its levels increase during this period ${ }^{31}$ and blastocyst implantation occurs when photoperiod is shortened (more hours of darkness than light during 24 hours), as has been reported for California sea $\operatorname{lion}^{32}$, so melatonin secretion has an effect on the hypothalamus-hypophysis-gonadal axis. As a result, in captive California sea lions between latitudes $21^{\circ} \mathrm{N}-43^{\circ} \mathrm{N}$, births occurs 0.6 days earlier for each degree of northward displacement, and the duration of the birthing season is more extended in low latitudes ${ }^{32}$. It has also been reported that seasonal changes in endocrine physiology, such as testosterone elevation prior to the reproductive season followed by elevation in cortisol levels induce territorial and maintenance of the harem behavior in Weddell seal males ${ }^{31}$.

Therefore a predictable environmental stimulus that may be influence the reproductive cycles of pinnipeds is the photoperiod, which determines the seasonal rhythms in most species that inhabit temperate and polar latitudes ${ }^{7}$.

\section{Photoperiod as modulating signal on reproductive cycles}

The photoperiod is defined as the proportion of light / dark hours in the 24-hour period. The behavioral and physiological patterns of organisms in response to the photoperiod are known as photoperiodism. This is a determining signal to define reproductive cycles of various species. Temte in 1985 and 1989, examined the reproductive season records of northern fur seal and the California sea lion in different latitudes and concluded that reproduction in both species is directly related to the animals' response to the photoperiod of 11.5 $12.5 \mathrm{~h}$, which occurs before implantation of the blastocyst. This suggests that the end of the inactive phase of the reproductive cycle is determined by the photoperiod ${ }^{10}$.

Another stimulus that determines the seasonality on pinnipeds is food availability, which in turn is associated to photoperiodism. The southern elephant seal feeds on free-ice pelagic waters during the summer, the dives during the day are deeper $(500-1500 \mathrm{~m})$ than at night $(150-300 \mathrm{~m})$, focusing the subsurface temperature and the maximum salinity characteristic of circumpolar deep water. This feeding pattern is probably in response to copepods vertical migration, base of the pelagic trophic chain ${ }^{33}$.

In conclusion, environmental conditions exhibit seasonal and latitudinal variations. Therefore the pinnipeds have developed various reproductive and feeding strategies to maximize resources and ensure their survival and reproductive success. The photoperiod is the main stimulus that shapes reproductive cycles of these organisms, since it has an effect on temperature and food availability.
On evolutive scale, environmental conditions also determine mating systems, if breeding site is a stable area, competition between males for females increases, consequently, they have a more marked sexual dimorphism than the species in which the competition for females is not so intense.

\section{ACKNOWLEDGMENTS}

We Thank to Biol. Roberto Velasco-González for their comments on the translation to English of this manuscript and MSc. Sergio Pérez-Galicia for the illustration of figure 2.

\section{REFERENCES}

1. Abadía-Cardoso A. Variabilidad genética del elefante marino del norte, Mirounga angustirostris, en Isla Guadalupe, Islas San Benito e Isla de Cedros, México. 2006.

2. Boness DJ, Bowen WD. The Evolution of Maternal Care in Pinnipeds: New findings raise questions about the evolution of maternal feeding strategies. Bioscience 1996;40:645-54.

3. Cullen TM, Fraser D, Rybczynski N, Schröder-Adams C. Early evolution of sexual dimorphism and polygyny in pinnipedia. Evolution (N Y) 2014;68:1469-84. https://doi.org/10.1111/evo.12360.

4. García-Aguilar MC, Elorriaga Verplancken FR. Los pinnípedos: carnívoros acuáticos altamente especializados. Ciencia 2019;70:72-9.

5. Boehme L, Baker A, Fedak M, Arthun M, Nicholls K, Robinson P, et al Bimodal winter haul-out patterns of adult Weddell seals (Leptonychotes weddellii) in the southern Weddell Sea. PLoS One 2016;11:1-19. https:/ / doi.org/10.1371/journal.pone.0155817.

6. Aguilar A, Cappozzo LH, Gazo M, Pastor T, Forcada J, Grau E. Lactation and mother-pup behaviour in the Mediterranean monk seal Monachus monachus: An unusual pattern for a phocid. J Mar Biol Assoc United Kingdom 2007;87:93-9. https://doi.org/10.1017/S0025315407056147.

7. Dardente H, Wood S, Ebling F, Sáenz de Miera C. An integrative view of mammalian seasonal neuroendocrinology. J Neuroendocrinol 2019;31:1_ 17. https://doi.org/10.1111/jne.12729.

8. Laws RM, Baird A, Bryden MM. Breeding season and embryonic diapause in crabeater seals (Lobodon carcinophagus). Reproduction 2003;126:365-70. https://doi.org/10.1530/rep.0.1260365.

9. Sjare B, Stirling I. The breeding behavior of Atlantic walruses, Odobenus rosmarus rosmarus, in the Canadian High Arctic. Can J Zool 1996;74:897-911. https://doi.org/10.1139/z96-103.

10. Kiyota Am, Tomita N, Baba N. Latitudinal Variation in Birth Dates of Northern Fur Seals (Callorhinus ursinus) in Captivity. Mammal Study 2009;34:231-5.

11. Boyd I. Environmental and physiological factors controlling the reproductive cycles of pinnipeds. Can J Zool 1991;69:1135-48. https:// doi.org/10.1139/z91-162.

12. Costa DP. Reproductive and Foraging Energetics of High Latitude Penguins, Albatrosses and Pinnipeds: Implications for Life History Patterns 1 1991;130:111-30.

13. Harcourt RG, Kingston JJ, Cameron MF, Waas JR, Hindell MA. Paternity analysis shows experience, not age, enhances mating success in an aquatically mating pinniped, the Weddell seal (Leptonychotes weddellii). Behav Ecol Sociobiol 2007;61:643-52. https://doi.org/10.1007/s00265006-0294-x.

14. Lindenfors P, Tullberg BS, Biuw M. Phylogenetic analyses of sexual selection and sexual size dimorphism in pinnipeds. Behav Ecol Sociobiol 2002;52:188-93. https://doi.org/10.1007/s00265-002-0507-x.

15. Sáenz De Miera C, Monecke S, Bartzen-Sprauer J, Laran-Chich MP, Pévet P, Hazlerigg DG, et al. A circannual clock drives expression of genes central for seasonal reproduction. Curr Biol 2014;24:1500-6. https:// doi.org/10.1016/j.cub.2014.05.024.

16. Duck CD. Annual variation in the timing of reproduction in Antarctic fur seals, Arctocephalus gazella, at Bird Island, South Georgia. J Zool 1990;222:103-16. https://doi.org/10.1111/j.1469-7998.1990. tb04032.x.

17. Pomeroy P. Reproductive cycles of marine mammals. Anim Reprod Sci 2011;124:184-93. https://doi.org/10.1016/j.anireprosci.2010.08.021.

18. Pharo EA, Cane KN, McCoey J, Buckle AM, Oosthuizen WH, Guinet $\mathrm{C}$, et al. A colostrum trypsin inhibitor gene expressed in the Cape fur seal mammary gland during lactation. Gene 2016;578:7-16. https://doi. org/10.1016/j.gene.2015.11.042. 
19. Stirling I. The social evolution of mating systems in pinnipeds. Recent Adv Study Mamm Behav 1983:489-527.

20. Shaughnessy PD, Southwell C. Are crabeater seals, Lobodon carcinophaga, sexually dimorphic for size during the breeding season? Mar Mammal Sci 2019;35:677-83. https://doi.org/10.1111/mms.12549.

21. Langley I, Fedak M, Nicholls K, Boehme L. Sex-related differences in the postmolt distribution of Weddell seals (Leptonychotes weddellii) in the southern Weddell Sea. Mar Mammal Sci 2018;34:403-19. https://doi. org/10.1111/mms.12461.

22. Baker JD, Thompson PM. Temporal and spatial variation in age-specific survival rates of a long-lived mammal, the Hawaiian monk seal. Proc R Soc B Biol Sci 2007;274:407-15. https://doi.org/10.1098/rspb.2006.3737.

23. Samaranch R, González LM. Changes in morphology with age in Mediterranean monk seals (Monachus monachus). Mar Mammal Sci 2000;16:141-57. https://doi.org/10.1111/j.1748-7692.2000.tb00909.x.

24. Szteren D, Aurioles D, Gerber LR. Population status and trends of the California sea lion (Zalophus californianus californianus) in the Gulf of California, Mexico. Sea Lions World 2006. https://doi.org/10.4027/slw.2006.25.

25. García-Aguilar M, Aurioles-Gamboa D. Breeding season of the California sea lion (Zalophus californianus) in the Gulf of California, Mexico. Aquat Mamm 2003;29:67-76. https://doi.org/10.1578/016754203101024086.

26. Pörschmann, U., Trillmich, F., Mueller, B., Wolf J. Male reproductive success and its behavioural correlates in a polygynous mammal, the Galápagos sea lion (Zalophus wollebaeki). Mol Ecol 2010:2574-86. https://doi.org/10.1111/j.1365-294X.2010.04665.x.
27. Born EW. Reproduction in female Atlantic walruses (Odobenus rosmarus) from north-west Greenland. J Zool 2001;255:165-74. https:// doi.org/10.1017/S0952836901001236.

28. Greig DJ, Mashburn KL, Rutishauser M, Gulland FMD, Williams TM, Atkinson S. Seasonal Changes in Circulating Progesterone and Estrogen Concentrations in the California Sea Lion (Zalophus californianus). J Mammal 2007;88:67-72. https://doi.org/10.1644/06-mamm-a060r2.1.

29. Horst-Werner K. Signaling pathways to and from the hypophysial pars tuberalis, an important center for the control of seasonal rhythms. Gen Comp Endocrinol 2018;258:236-43. https://doi.org/10.1016/j. ygcen.2017.05.011.

30. Bennett K, McConnell B, Fedak MA. Diurnal and seasonal variations in the duration and depth of the longest dives in Southern Elephant seals (Mirounga leonina): Possible physiological and behavioural constraints. J Exp Biol 2001;204:649-662.

31. Atkinson S. Reproductive biology of seals. Rev Reprod 2004;2:175-94. https://doi.org/10.1530/revreprod/2.3.175.

32. Temte JL, Temte J. Photoperiod defines the phenology of birth in cpative California sea lions. Mar Mammal Sci 1993;9:301-8.

33. Biuw M, Nøst OA, Stien A, Zhou Q, Lydersen C, Kovacs KM. Effects of hydrographic variability on the spatial, seasonal and diel diving patterns of Southern Elephant seals in the Eastern Weddell sea. PLoS One 2010;5. https://doi.org/10.1371/journal.pone.0013816. 


\section{EEG power spectrum daily variations in sleep and wakefulness}

Lucía Osorio $^{1}$

Alejandra Mondino ${ }^{1,2}$

Matías Cavelli ${ }^{1,3}$

Joaquín González

Pablo Torterolo ${ }^{1}$

Alicia Costa ${ }^{1 *}$

${ }^{1}$ Laboratorio de Neurobiología del Sueño, Departamento de Fisiología, Facultad de Medicina, Universidad de la República, Montevideo, Uruguay.

${ }^{2}$ Department of Anesthesiology, University of Michigan, Ann Arbor, MI, USA.

${ }^{3}$ Department of Psychiatry, University of Wisconsin, Madison, WI, USA.

\begin{abstract}
Objective: To study the differences in electrocortical activity during wakefulness (W), NREM and REM sleep throughout the subjective day and night of the rat. Methods: 24-hours of continuous polysomnographic recordings were performed on seven male rats, using neocortical superficial electrodes in a $12 \mathrm{~h}$ light $/ 12 \mathrm{~h}$ dark condition. The daily variation of the power spectrum $(0.1-200$ $\mathrm{Hz}$ ) was analyzed for each behavioral state. Results: In comparison to the dark phase, W during the day was characterized by increases in the relative power of frequencies slower than $30 \mathrm{~Hz}$ while higher frequencies were lower. NREM sleep showed marked increases in frequencies higher than $20 \mathrm{~Hz}$ during the night in comparsion with the light phase; while the delta band $(0.5-4 \mathrm{~Hz})$ was prominent in diurnal NREM. While the relative power spectrum of REM sleep was homogeneous during the day, it variates in a complex manner during the night. Discussion: Electrocortical EEG profile of $\mathrm{W}$ and sleep is highly dependent on the time of the day.
\end{abstract}

Keywords: Rem Sleep; NREM; EEG; Circadian; Rhythm; Delta; Gamma 


\section{INTRODUCTION}

The sleep-wake cycle is a critical physiological process and one of the most preserved biological rhythms throughout evolution. This cycle is composed of different behavioral states, commonly distinguished by their electrophysiological signatures and behavioral characteristics. These states correspond to wakefulness (W), non-rapid eye movement (NREM) sleep and rapid eye movement (REM) sleep. W and sleep are associated with different brain functional states, which can be captured by electroencephalographic (EEG) signals containing a broad frequency spectrum.

The EEG reflects the interrelated multicomponent activity of numerous cortical and subcortical neuronal ensembles ${ }^{1}$. EEG activity consists of various frequency bands that vary during the sleep-wake cycle in a characteristic fashion. In the rat, the EEG during W is characterized by low voltage fast waves as well as theta rhythm $(4.5-9 \mathrm{~Hz})$ in posterior cortices mainly during active W. NREM sleep EEG is recognized by the occurrence of high-amplitude slow waves $(0.5$ to $4 \mathrm{~Hz})$ and electrographic events of $0.5-2$ seconds in duration known as sleep spindles, that have an intra-event frequency of $9-15 \mathrm{~Hz}^{2,3}$. EEG during REM sleep shows a high-frequency rhythm that is nested in very regular and prominent theta activity ${ }^{4,5}$.

In contrast to humans, rodents show a strong ultradian component in their sleep, with no single period of consolidated W during the active (night) period or consolidated sleep during the rest (diurnal) period ${ }^{6}$. This polyphasic nature of their sleepwake cycle allows us to study the features of the EEG during W, NREM and REM sleep at different times of the day.

Previous works in rodents and humans have shown the existence of diurnal variations in the EEG activity at lowfrequency bands (up to $30 \mathrm{~Hz}$ ) (i.e. 7, 2, 8, 9). However, to the best of our knowledge, a continuous recording and analysis of the diurnal variations of the high-frequency bands of the EEG are lacking. Hence, the aim of this report is to convey an initial descriptive analysis of the changes in the EEG power spectra (0.1-200 Hz) of sleep and W occurring at different times of the day. For this purpose, we performed an hour-to-hour analysis of the power spectrum in each behavioral state over a $24 \mathrm{~h}$ period and illustrate it as the Z-score variation from the mean.

\section{MATERIAL AND METHODS}

\section{Experimental animals}

Seven adults male Wistar rats (275-330 g) were used in this study. The animals were obtained from and determined to be in good health by the Institutional Animal Care Facility. The experimental procedures were conducted in accordance with the Guide for the Care and Use of Laboratory Animals (8th edition, National Academy Press, Washington, DC, 2010) and approved by the Institutional Animal Care Commission (protocol No. 070153000332-16, Facultad de Medicina, Universidad de la República). Adequate measures were taken to minimize pain, discomfort or stress of the animals, and efforts were made to use the smallest number of animals necessary to obtain reliable data.

\section{Surgical procedures}

The animals were chronically implanted with electrodes to monitor the states of sleep and W. We employed surgical procedures like those used in our previous studies (e.g., 10). Anesthesia was induced with a mixture of ketamine-xylazine (90 mg/kg; $5 \mathrm{mg} / \mathrm{kg}$ i.p., respectively). The rat was positioned in a stereotaxic frame, and the skull was exposed. To record the EEG, stainless steel screw electrodes (1 $\mathrm{mm}$ diameter) were screwed on craniotomies to have their tips touching the brain's surface (above the dura mater) in different cortices. The arrangement of electrodes is depicted in Figure 1. Six electrodes were located bilaterally in the primary motor cortex (M1: $\mathrm{L} \pm 2.5 \mathrm{~mm}, \mathrm{AP}+2.5 \mathrm{~mm}$ ), the primary somatosensory cortex (S1: $\mathrm{L} \pm 2.5 \mathrm{~mm}, \mathrm{AP}-2.5 \mathrm{~mm})$ and secondary visual cortex (V2: $\mathrm{L} \pm 2.5 \mathrm{~mm}, \mathrm{AP}-7.5 \mathrm{~mm})$. The reference electrode was placed in the cerebellum (Figure $1 \mathrm{~A}$ ). Bipolar electrodes were inserted into the neck muscles in order to record the electromyogram (EMG). The electrodes were connected to a plug that was bonded to the skull with acrylic cement. At the end of the surgical procedures, an analgesic (Ketoprofen, $1 \mathrm{mg}$ / $\mathrm{kg}$, subcutaneously) was administered. Incision margins were kept clean and a topical antibiotic was applied daily. After the animals recovered from the preceding surgical procedures, they were adapted to the recording chamber for one week.

\section{Experimental sessions}

All animals were housed individually in transparent cages $(40 \times 30 \times 20 \mathrm{~cm})$ containing wood shavings in a temperaturecontrolled room $\left(21-24^{\circ} \mathrm{C}\right)$ with $12: 12 \mathrm{~h}$ light-dark cycle, light $(50$ lux) beginning at 08:00h (ZT0), with water and food ad libitum. The recordings were performed through a rotating connector, to allow the rats to move freely within the recording box.

All the data was collected continuously over a $24 \mathrm{~h}$ period recordings starting at ZTO. Bioelectric signals were amplified $(\times 1000)$, the filters were set at $0.1 \mathrm{~Hz}$ and $200 \mathrm{~Hz}$. EEG and EMG activity were captured and stored directly on a PC computer through a National Instruments data acquisition card of 16 bits with a sampling rate of $1024 \mathrm{~Hz}$ by means of DASYLab software (Measurement Computing). The animals were left undisturbed during all the recordings.

\section{Data analysis}

Behavioral states were determined in 10 s epochs. W was defined as low voltage fast waves in the frontal cortex, sometimes with theta rhythm in occipital cortex and relatively high EMG activity. Light sleep (LS) was defined as high voltage slow cortical waves interrupted by low voltage fast electroencephalographic activity, while slow-wave sleep (SWS) was identified by continuous high amplitude slow (1-4 Hz) frontal, parietal and occipital waves and sleep spindles combined with a reduced EMG activity. For the daily power spectrum analysis, LS and SWS were pooled together and classified as NREM sleep. REM sleep was defined as low voltage fast frontal waves, a regular theta rhythm in the parietal and occipital cortices, and a silent EMG except for occasional myoclonic twitching. 
A)

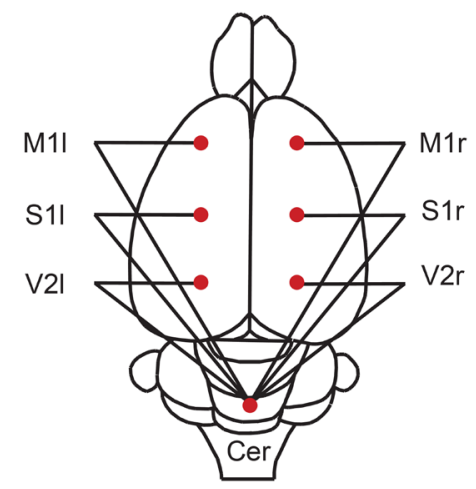

C)
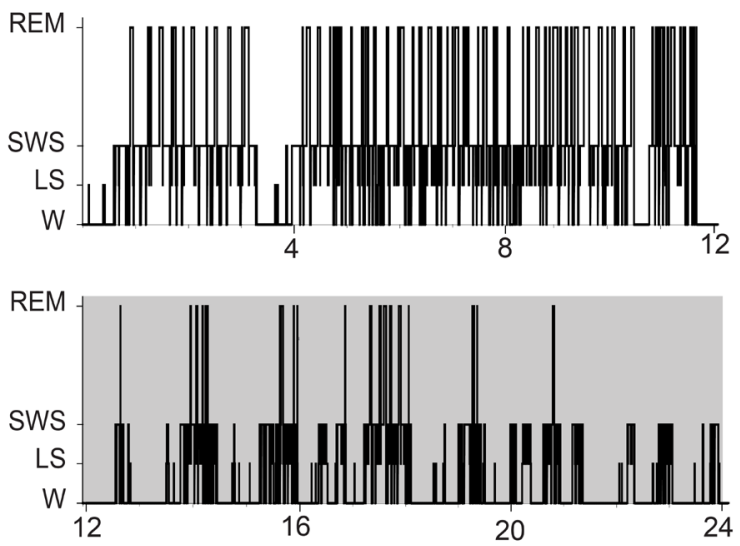

B)

Wakefulness

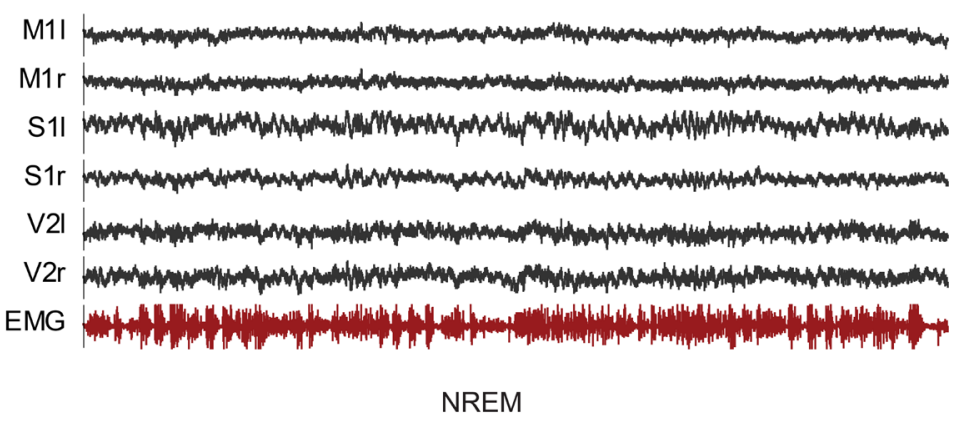

M11 fom M1r

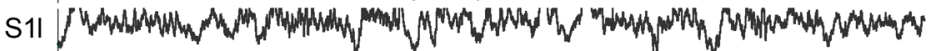

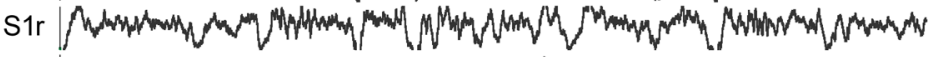
V21

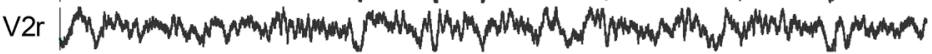
EMG

REM

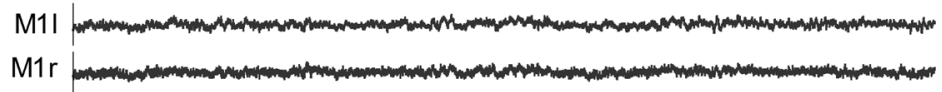

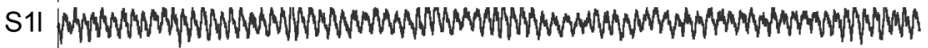

S1r Mww

V21

V2r AHW EMG

Figure 1. A) Representative scheme showing the position of the recording electrodes. The electrodes were referred to a common electrode that was located over the cerebellum (Cer). M1, primary motor cortex; S1, primary somatosensory cortex; V2, secondary visual cortex; r, right; 1, left. B) Polysomnographic recordings of a representative animal in wakefulness, NREM sleep, and REM sleep. EMG: electromyogram. C) Representative hypnogram of the 24h period (0 is 8:00 AM: ZT0). The shaded area indicates the lights-off period.

In order to analyze the power spectrum in each EEG channel, we used procedures like those applied in our previous studies (e.g., 10). The power spectrum was estimated on Matlab using the Welch function (Hamming window, window size 10 s, with an overlap of $2.5 \mathrm{~s}$, a frequency sample of $1024 \mathrm{~Hz}$ and a resolution of $0.5 \mathrm{~Hz}$ ). The mean power of all 6 electrodes and 7 rats, was used to calculate the relative power. This was determined by dividing the power value for each frequency band in each time point, by the sum of the total power for that frequency in the $24 \mathrm{hs}$. $Z$ transformations were made in order to express the values as deviations from the mean. It is important to note that in the REM sleep analysis the last hour was not computed due to a coincidental lack of this behavioral state in all animals during that period.

\section{RESULTS}

Polysomnographic recordings

The simultaneous electrocortical activity of different brain areas was recorded continuously over a $24 \mathrm{~h}$ period.
Representative recordings of these areas during W, NREM, and REM sleep are shown (Figure 1B). The polycyclic nature of the sleep-wake cycle of the rat can be observed in a representative hypnogram (Figure 1C). The animals showed a clear preference for sleep during the light period (72\% of total sleep time; $59 \%$ in NREM and $13 \%$ in REM sleep) and to be awake at night (61\% in W; $32 \%$ in NREM and $4 \%$ in REM sleep).

Daily power spectrum during wakefulness

Figure 2A shows the hour-to-hour analysis of the percentage of $\mathrm{W}$; the daily variation in the percentage of $\mathrm{W}$ is readily observed.

The EEG power spectrum during W has robust daily variations, as shown in the spectrograms (grand average of animals and channels) (Figure 2B and C). The main observation was that diurnal and nocturnal $\mathrm{W}$ differ in their electrocortical activity profiles. The relative power of the high-frequency rhythms $(30-200 \mathrm{~Hz})$ was higher both at the beginning (between ZT12 and ZT16) and at the end of the dark phase, reaching values that were 2 to 3 standard deviations (SD or Z-units) over the mean (Figure 2B). 


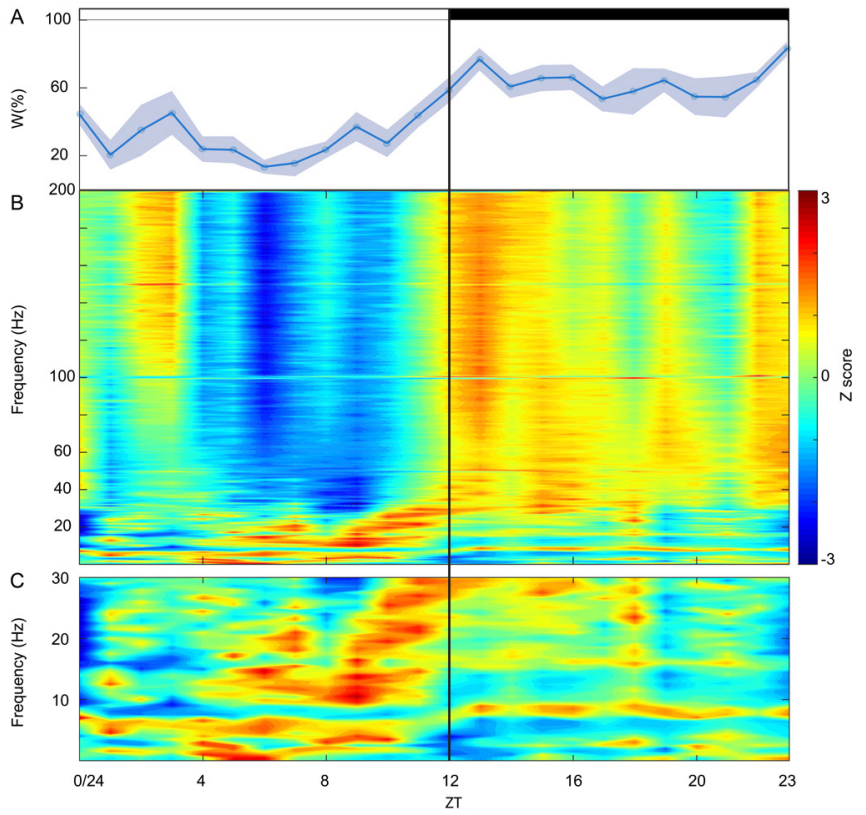

Figure 2. EEG relative power spectra during wakefulness. A) Percentage of time spent in W throughout the $24 \mathrm{~h}$ period. The shaded outline indicates $1 \mathrm{SD}$. The top bar indicates light and dark periods. B) Z-score of the relative power spectrogram of $\mathrm{W}$ during the $24 \mathrm{~h}$ period. The black vertical line indicates lights-off onset. C) Zoomed inset showing the $\mathrm{Z}$-score of the relative power spectrogram for the $0-30 \mathrm{~Hz}$ band. ZT0 $=8 \mathrm{AM}$. Panels $\mathrm{B}$ and $\mathrm{C}$ share the same $\mathrm{Z}$-score color scale. All Y-axis starting at 0 .

In addition, their lower values were observed in the ZT4-ZT10 window (light phase). Inspecting the frequencies up to $30 \mathrm{~Hz}$ we observed higher relative power values ( 2 to 3 SD over the mean) mainly from Z4 up to Z12 (Figure 2C). Note that from ZT8 to ZT12 there is a progressive increase in the relative power of frequencies in the $12-30 \mathrm{~Hz}$ range. Another interesting observation is that theta-band activity ( 5 to $9 \mathrm{~Hz}$ ) seemed to be faster during the nighttime.

\section{Daily power spectrum during NREM sleep}

As it is shown in Figure 3A NREM sleep predominates during the light phase. During the light phase, from ZT0 to ZT6, NREM was characterized by an increase in the relative power of the activity up to $5 \mathrm{~Hz}$ (mainly slow-wave activity, SWA), and a decrease in the higher frequencies (Figure $3 \mathrm{~B}$ and $\mathrm{C}$ ). From ZT8 a progressive increase in power in 9-15 Hz oscillations (sleep spindles) was observed. The SWA relative power reached a minimum value two hours before lights-off. During the dark period, NREM was characterized by an increase in the relative power of frequencies higher than 6 $\mathrm{Hz}$ and up to $200 \mathrm{~Hz}$ (Figure $3 \mathrm{~B}$ and C); however, these changes were not homogenous throughout the night.

\section{Daily power spectrum during REM sleep}

Figure 4A shows the hour-to-hour analysis of the percentage of REM sleep. This behavioral state was more prominent during the light phase, no presence of REM sleep was detected in the last hour of the recordings.

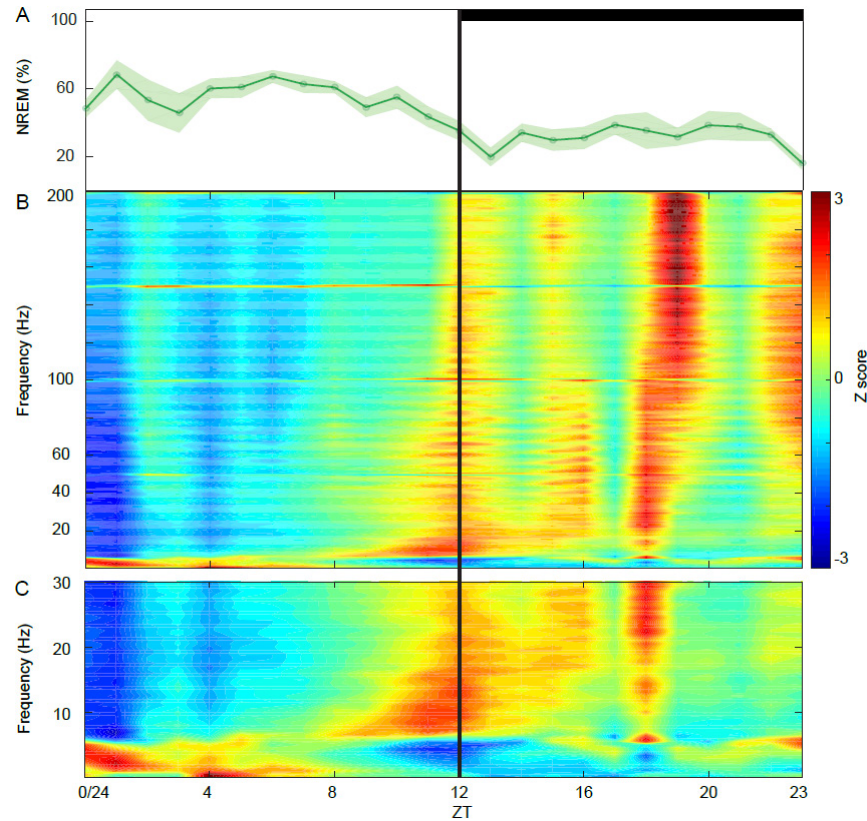

Figure 3. EEG relative power spectra during NREM sleep. A) Top panel: Percentage of time spent in NREM throughout the $24 \mathrm{~h}$ period. The shaded outline indicates $1 \mathrm{SD}$. The top bar indicates light and dark phases. B) Z-score for the relative power spectrogram of NREM during the $24 \mathrm{~h}$ period. The black vertical line indicates lights-off onset. C) Zoomed inset showing the Z-score of the relative power spectrogram for the $0-30 \mathrm{~Hz}$ band. ZT0 $=8 \mathrm{AM}$. Panels B and $\mathrm{C}$ share the same $\mathrm{z}$-score color scale. All Y-axis starting at 0 .

The relative power spectrogram during the lights-on period showed no marked changes in relation to the mean (Figure 4B); however, it was highly variable during the dark phase.

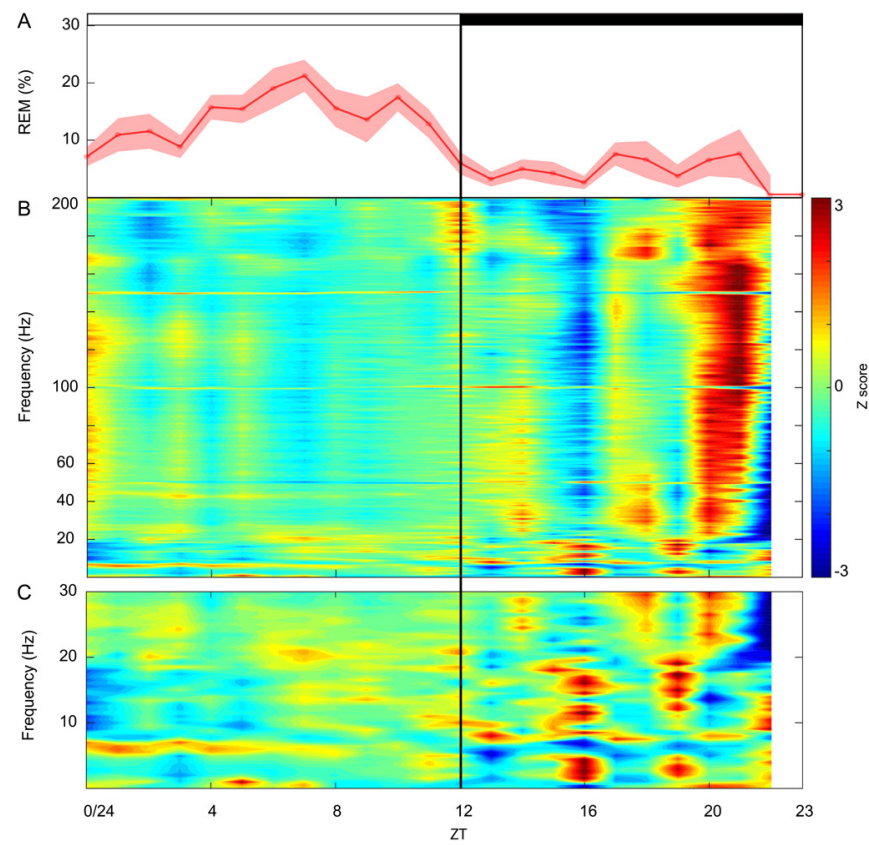

Figure 4. EEG relative power spectra during REM sleep. A) Percentage of time spent in REM throughout the $24 \mathrm{~h}$ period. The shaded outline indicates $1 \mathrm{SD}$. The top bar indicates light and dark phases. B) Z-score for the relative power spectrogram during the $24 \mathrm{~h}$ period. The black vertical line indicates lights-off onset. C) Zoomed inset showing the Z-score of the relative power spectrogram for the $0-30 \mathrm{~Hz}$ band. $\mathrm{ZT} 0=8 \mathrm{AM}$. Panels B and C share the same $\mathrm{z}$-score color scale. All Y-axis starting at 0 . 
In fact, the relative power of frequencies up to $30 \mathrm{~Hz}$ changed with short time increases and decreases over the mean. Also, the most remarkable fact was the increase in the relative power of frequencies in the $20-200 \mathrm{~Hz}$ range, at the end of the nighttime (Figure 4B). During the day, theta frequency appears to be slower than during the dark- phase. (Figure 4C). Note that the last hour in the spectrogram is blank due to the lack of REM sleep during this period.

\section{DISCUSSION}

In this preliminary study, we performed a descriptive analysis of the daily variations in the EEG power spectrum $(0.1-200 \mathrm{~Hz})$ of W, NREM and REM sleep of the rat. We demonstrated the presence of an important daily variation in the spectrographic profile of both $\mathrm{W}$ and sleep. This variation was evident both within and between the light and dark phases.

Previous studies showed diurnal variations in the EEG of the rat on frequencies up to $30 \mathrm{~Hz}^{7,4,11}$. In fact, our results support the pioneer studies of Rosenberg et al., ${ }^{12}$ and Steinfels et al. ${ }^{7}$, that showed that the highest spectral values can be found during the day in the delta band for NREM sleep and in the theta band for REM sleep. However, we chose to use relative power in order to analyze differences within each frequency band throughout the $24 \mathrm{hs}$ for each behavioral state. These previous studies were limited to study frequencies lower than $30 \mathrm{~Hz}$, while in this report we also analyze the higher frequencies (up to $200 \mathrm{~Hz}$ ).

Our results showed that diurnal W was heterogeneous with the main changes being a decrease in the relative power of high frequency oscillations $(>30 \mathrm{~Hz}$ ), and bouts of increase in the relative power of slower rhythms. Nocturnal W was characterized by a larger relative power in the high-frequency rhythms ( $>30 \mathrm{~Hz}$ ) while slower oscillations, apart from the theta band, showed not main changes. This result suggests that nocturnal W presents greater cortical arousal than diurnal W.

Human electrocortical activity has been shown to exhibit important diurnal and circadian variations. Cacot et al. ${ }^{13}$ found a clear diurnal variation in the power of EEG rhythms up to $30 \mathrm{~Hz}$ during W, with maximum achieved at noon or in the afternoon. In addition, classic studies by Kleitman have shown ultradian differences in behavior during $\mathrm{W}$, what was called basic restactivity cycle ${ }^{14}$; however, the electrophysiological counterpart of this ultradian cycle has not been studied in detail.

Regarding NREM sleep, in comparison to nighttime, diurnal activity presented a higher power in lower frequencies (mainly below $5 \mathrm{~Hz}$ ), while the power of the high frequencies ( $>$ $30 \mathrm{~Hz}$ ) was lower. Hence, suggesting that NREM sleep during the day is deeper than during the night. In accordance with Bergmann et $\mathrm{al}^{2}$, our data showed that as NREM sleep time increases, slow-wave incidence and amplitude increase. Fluctuations in the elctrocortical activity, probably reflecting the level of arousal, also occur during night NREM sleep. In humans, a cyclic alternating pattern (CAP), characterized by the regular alternation of EEG patterns that represents a complex form of periodic activity, was described during NREM sleep see ${ }^{15,16}$. In fact, Terezano et al. ${ }^{17}$, showed the existence of a CAP in NREM sleep characterized by changes at the level of arousal to sensory stimuli.
An important fact, is that during W and NREM, an opposite correlation between high frequency $(>30 \mathrm{~Hz})$ and low-frequency oscillations (mainly in the delta, 0.5-4 Hz band) was evident. This result agrees with Maloney et al. ${ }^{18}$ who showed that gamma activity was negatively correlated with delta across all behavioral states.

Regarding REM sleep, our results showed a mostly homogeneous profile during the light-phase. This is in accordance with previous results by Borbély et al. ${ }^{4}$, who showed no clear variations in the relative EEG power density of REM during the day. Hence, REM sleep is a highly stable behavioral state during the light phase. During the night REM sleep the spectrogram has a changing profile with alternations between bouts of slow $(<20 \mathrm{~Hz})$ and high $(30-200 \mathrm{~Hz})$ electrocortical activity. Our results agree with those of Steinfels et al. ${ }^{7}$ where a clear increase in the EEG spectral power was observed at the end of the dark period. Finally, it was readily observed that during the first half of the light period, the frequency of the peak of the theta band during REM sleep was lower that in the dark period; probably this fact could be related with a more active REM sleep during the night ${ }^{19}$.

\section{CONCLUSIONS AND FUTURE DIRECTIONS}

In the present report, we demonstrated a clear difference in the power spectrum profile both in W and sleep during the light and dark phases among a wide range of electrocortical oscillations (0.1-200 Hz). However, further analysis needs to be done in order to better understand if the observed variations depend on external cues, circadian and/or homeostatic process (processes C and S, respectively; see Borbély et al., 20). Sleep deprivation, as well as recording in free-running conditions, will be optimal to answer these interrogations.

In addition, another future direction is to perform a detailed analysis of the daily variation of the activity of different neocortical and archicortical (olfactory bulb) areas as well as of their functional connectivity.

\section{ACKNOWLEDGMENTS}

This work was supported by Agencia Nacional de Investigación e Innovación (ANII), (Proyecto FCE_1_2017_1_136550); Comisión Sectorial de Investigación Científica (CSIC) (Proyecto I+D 2016_589); and Programa de Desarrollo de Ciencias Básicas (PEDECIBA)-Uruguay.

\section{REFERENCES}

1. da Silva FL. Neural mechanisms underlying brain waves: from neural membranes to networks. Electroencephalography and clinical neurophysiology. 1991 Aug 1;79(2):81-93.

2. Bergmann BM, Winter JB, Rosenberg RS, Rechtschaffen A. NREM sleep with low-voltage EEG in the rat. Sleep. 1987 Jan 1;10(1):1-1.

3. Etevenon P, Giannella F. Waking and sleeping states in the rat from an EEG data analysis point of view. Waking \& Sleeping. 1980 JanMar;4(1):33-45.

4. Borbély AA, Tobler I, Hanagasioglu M. Effect of sleep deprivation on sleep and EEG power spectra in the rat. Behavioural brain research. 1984 Dec 1;14(3):171-82

5. Young GA, Steinfels GF, Khazan N, Glaser EM. Cortical EEG power spectra associated with sleep-awake behavior in the rat. Pharmacology Biochemistry and Behavior. 1978 Jan 1;8(1):89-91. 
6. Yasenkov R, Deboer T. Circadian modulation of sleep in rodents. In: Progress in brain research 2012 Jan 1 (Vol. 199, pp. 203-218). Elsevier.

7. Steinfels GF, Young GA, Khazan N. Diurnal variations in REM and NREM sleep EEG power spectra in the rat. Brain research. 1980 Jan 13;181(2):425-32.

8. Grasing K, Szeto H. Diurnal variation in continuous measures of the rat EEG power spectra. Physiology \& behavior. 1992 Feb 1;51(2):249-54.

9. Dijk DJ. Circadian variation of EEG power spectra in NREM and REM sleep in humans: dissociation from body temperature. Journal of sleep research. 1999 Sep;8(3):189-95.

10. Cavelli M, Castro-Zaballa S, Mondino A, Gonzalez J, Falconi A, Torterolo P. Absence of EEG gamma coherence in a local activated cortical state: a conserved trait of REM sleep. Translational Brain Rhythmicity. 2017;21132017.

11. Grasing K, Szeto H. Diurnal variation in continuous measures of the rat EEG power spectra. Physiology \& behavior. 1992 Feb 1;51(2):249-54.

12. Rosenberg RS, Bergmann BM, Rechtschaffen A. Variations in slow wave activity during sleep in the rat. Physiology \& behavior. 1976 Dec 1;17(6):931-8.

13. Cacot P, Tesolin B, Sebban C. Diurnal variations of EEG power in healthy adults. Electroencephalography and clinical neurophysiology. 1995 May 1;94(5):305-12
14. Kleitman N. The basic rest-activity cycle and physiological correlates of dreaming. Experimental neurology. 1967 Dec 1;19:2-4.

15. Gaches J. Activités périodiques en EEG. Revue d'Electroencéphalographie et de Neurophysiologie Clinique. 1971 Jan 1;1(1):9-33.

16. Terzano MG, Mancia D, Manzoni Gc. Periodic activities in CreutzfeldtJakob disease and epilepsy. In: Nisticb G, Di Perri R, Meinardi H, eds. Epilepsy: an update on research and therapy. New York: Alan R. Liss, 1983:199-226.

17. Terzano MG, Mancia D, Salati MR, Costani G, Decembrino A, Parrino L. The cyclic alternating pattern as a physiologic component of normal NREM sleep. Sleep. 1985 Jun 1;8(2):137-45.

18. Maloney KJ, Cape EG, Gotman J, Jones BE. High-frequency $\gamma$ electroencephalogram activity in association with sleep-wake states and spontaneous behaviors in the rat. Neuroscience. 1997 Jan $1 ; 76(2): 541-55$

19. Sei H, Morita Y. Acceleration of EEG theta wave precedes the phasic surge of arterial pressure during REM sleep in the rat. Neuroreport. 1996 Nov 25;7(18):3059-62.

20. Borbély AA, Daan S, Wirz-Justice A, Deboer T. The two-process model of sleep regulation: a reappraisal. Journal of sleep research. 2016 Apr;25(2):131-43. 


\section{Chikungunya infection modulates the locomotor/flight activity of Aedes aegypti}

Karine Pedreira Padilha ${ }^{1}$ Octavio A. C. Talyuli ${ }^{2}$

Ricardo Lourenço-de-Oliveira ${ }^{3,4}$ Luana Cristina Farnesi ${ }^{1}$

Rafaela Vieira Bruno ${ }^{* *}$

\footnotetext{
${ }^{1}$ Fundação Oswaldo Cruz - Fiocruz, Instituto Oswaldo Cruz, Laboratório de Biologia Molecular de Insetos, Rio de Janeiro, RJ, Brazil.

${ }^{2}$ Universidade Federal do Rio de Janeiro, Instituto de Bioquímica Médica Leopoldo de Meis, Laboratório de Bioquímica de Insetos Hematófagos, Rio de Janeiro, RJ, Brazil.

${ }^{3}$ Fundação Oswaldo Cruz-Fiocruz, Instituto Oswaldo Cruz, Laboratório de Mosquitos Transmissores de Hematozoários, Rio de Janeiro, RJ, Brazil. ${ }^{4}$ Conselho Nacional de Desenvolvimento Científico e Tecnológico, Instituto Nacional de Ciência e Tecnologia em Entomologia Molecular, Brazil.
}

\begin{abstract}
Aedes aegypti mosquito is involved in the transmission of arboviruses such as chikungunya virus (CHIKV). The effects of CHIKV infection on the locomotor/flight behavior of the Ae. aegypti mosquito vector was not studied so far, although it represents an essential aspect of virus epidemiology. Here, locomotion/flight activity of infected females were for the first time evaluated by monitors that captured movement by infrared light beams for seven days under light/dark regimen (12 hours of light followed by 12 hours of dark) at $25^{\circ} \mathrm{C}$ and $60-80 \%$ relative humidity $(\mathrm{RH})$. The results showed that the CHIKV infection caused a significant decrease in the locomotion/ flight pattern of $A$ e. aegypti females. It describes an important difference in $A$ e. aegypti behavior and parasite-vector interaction, which may influence CHIKV viral spread and transmission dynamics. Thus, it is of great importance further studies focused on the analysis of other aspects of potential changes on physiology and behavior in infected mosquitoes.
\end{abstract}

Keywords: Aedes Aegypti; Chikungunya Virus; Locomotor Activity; Flight Activity

\author{
Corresponding author: \\ Rafaela Vieira Bruno \\ E-mail: rafaelav@ioc.fiocruz.br \\ rafaelabruno@gmail.com
}




\section{INTRODUCTION}

Aedes aegypti is a mosquito that belongs to the Culicidae family and the Aedes genus. This species can be found mainly in tropical and subtropical regions and its life cycle is divided into four stages: egg, four larval instars, pupae and adult. Female mosquitoes are hematophagous insects, feeding preferably on human blood, which is necessary for the maturation of their eggs. Ae. aegypti is capable to transmit several pathogens during the blood meal, being the most important vector of arboviruses responsible for serious public health problems, such as dengue (DENV), Zika (ZIKV), yellow fever and chikungunya $a^{1-3}$.

Chikungunya virus (CHIKV) belongs to Togaviridae family and Alphavirus genus. This virus was isolated for the first time in 1952 in Tanzania from human serum ${ }^{4}$. Following the occurrence of several isolated outbreaks of this Alphavirus around the world, CHIKV reached the Americas in 2013 through the Caribbean and in 2014, the first records of autochthonous transmission occurred in Brazil. Since then, the virus spread throughout causing serious epidemics ${ }^{5,6}$.

There are some vaccines that are yet in initial testing phase $^{7}$, thus the only way to try to contain CHIKV epidemics is focusing efforts on vector control. For this reason, understanding aspects of the biology and behavior of Ae. aegypti is highly encouraged.

In this sense, regarding the behavioral characteristics of this vector and the insects in general, it is known that the circadian clock is involved in the control of behaviors such as locomotion, flight, hematophagy, oviposition activity, among others $^{8}$. More specifically, concerning locomotor activity, it was seen that mosquito species could be classified as having diurnal, twilight and nocturnal habits. The Ae. aegypti mosquito has a diurnal and twilight pattern ${ }^{8,9}$.

Recently, studies have revealed that infection can modulate the locomotor behavior of Ae. aegypti. Infection by DENV2 in Ae. aegypti females caused them to increase their locomotor activity during the $24 \mathrm{~h}$ period under LD 12:12 regimen (LD, which is 12 hours of light followed by 12 hours of dark $)^{10}$. However, ZIKV showed an opposite effect; our group observed that ZIKV infection caused a decrease in locomotor/ flight activity of Ae. aegypti females in a LD 12:12 and constant dark (DD) regimen ${ }^{11}$. Besides influencing the locomotor behavior, it was observed that arboviruses could modulate other aspects of behavior, such as oviposition ${ }^{11,12}$. Thus, the next step, now, is to focus efforts on studies that describe the behavioral characteristics of this vector when infected with other circulating arboviruses, such as CHIKV.

Here, we aimed to study the effect caused by CHIKV infection on the behavior of Ae. aegypti locomotor activity/ flight in the LD regimen. This knowledge may serve for better understanding the transmission dynamics of this arbovirus.

\section{METHODS}

\section{Mosquito breeding}

All assays were performed with Aedes aegypti PAEA strain (Tahiti, French Polynesia). Mosquito breeding was realized in a controlled manner in the laboratory during all stages of its life cycle in incubators (Forlab Scientific Incubator, USA $)$ at $25^{\circ} \mathrm{C}\left( \pm 1^{\circ} \mathrm{C}\right)$ and $60-80 \% \mathrm{RH}$, under $\mathrm{LD}$ 12:12 regimen, details $i^{11}$. After the emergence of the adults, the females were kept together with the males for about 5 days to ensure insemination.

\section{Blood Feed/Viral Infection}

Aedes aegypti females were kept fasted for 6 hours before blood supply (infected with CHIKV or uninfected). Artificial feeding was done for approximately $40 \mathrm{~min}$ through a membrane attached to an artificial feeder at $37^{\circ} \mathrm{C}$.

The infectious blood meal consisted of a 1:1 mixture of rabbit red blood cells and L-15 culture medium containing CHIKV (isolate BHI3745/H804709, as described in ${ }^{5}$ ) with a final concentration of $10^{7} \mathrm{PFU} / \mathrm{ml}$; ATP was also included as phage stimulant at $\mathrm{pH} 7.4$ in a final concentration of $1 \mathrm{mM}$. Uninfected control mosquitoes were fed with naïve blood supplemented with L-15 culture media. Uninfected blood meal consisted of the same mixture but deprived of CHIKV.

After feeding, females were cold anesthetized and only the fully engorged were considered for the experiment (details $\left.i^{11,13}\right)$. The entire procedure was performed within a biosafety level 2 insectary facility (BSL-2, Laboratório de Bioquímica de Insetos Hematófagos, Instituto de Bioquímica Médica, UFRJ).

\section{Locomotor/flight activity}

After blood feeding, infected and uninfected Ae. aegypti females were individually transferred into $25 \mathrm{~mm}$ glass tubes. In all tubes we added cotton soaked with $10 \%$ sugar solution to ensure mosquitoes feeding during the experiment. The tubes were then positioned within locomotor/flight activity monitors (Trikinetics Inc, Waltham, MA, USA). Each monitor has 32 channels with infrared light beams and the activity is captured every time the mosquito interrupts the beam. In general, two monitors were used for each condition (infected and uninfected) per experiment. We performed three independent experiments totaling $144 \mathrm{CHIKV}$-infected and 139 uninfected mosquitoes. The monitors were placed inside incubators (ELETROlab Scientific Incubator, Brazil), with the constant temperature $\left(25^{\circ} \mathrm{C} \pm 1{ }^{\circ} \mathrm{C}\right)$, the humidity ranged from 60 to $80 \%$, in LD12:12.

The movement of mosquitoes was captured every 5 minutes, for seven sequential days. For better representativeness, data were transformed to 30 minutes to the analyzes. Mosquitoes that had no activity during the last 24 hours of the experiment were considered dead and disregarded from analyzes. 


\section{Data analysis}

All results were analyzed with Excel software (Microsoft Office). We represented the data compiled from values of Williams' mean ${ }^{14}$ every 30-minutes of activity of each day of the experiment. The data were transformed into logarithmic due to a large variation in individual mosquito values, thus avoiding data masking (as explained by ${ }^{11}$ ).

Statistical analysis was based on the methodology described by $^{11}$. Firstly, were performed the Shapiro-Wilk test to assess whether data is parametric or nonparametric. The data were analyzed for significance through the Mann Whitney statistical test.

\section{Ethical Statement:}

All experiments performed in this work were approved by Research Ethics Committees CEUA-UFRJ 149/19 (for rabbit blood use).

\section{RESULTS}

\section{CHIKV infection decreases females Ae. aegypti locomotor/ flight activity}

We performed experiments to determine if CHIKV infection influenced Ae. aegyti behavior. Figure 1 show the locomotor/flight activity where data were represented by the values of 30 -minutes mean activity of each experiment's day. As previously reported ${ }^{9,10}$, females of Ae. aegypti concentrate most activity during the light phase. A bimodal pattern of activity with a peak during the day (between ZT8 and ZT11) and a peak in the early evening (ZT13.5) were observed in both CHIKV-infected and uninfected mosquitoes. Moreover, as expected, after ZT14, mosquito's activity is drastically reduced, remaining close to zero until late in the evening ${ }^{9-11}$.

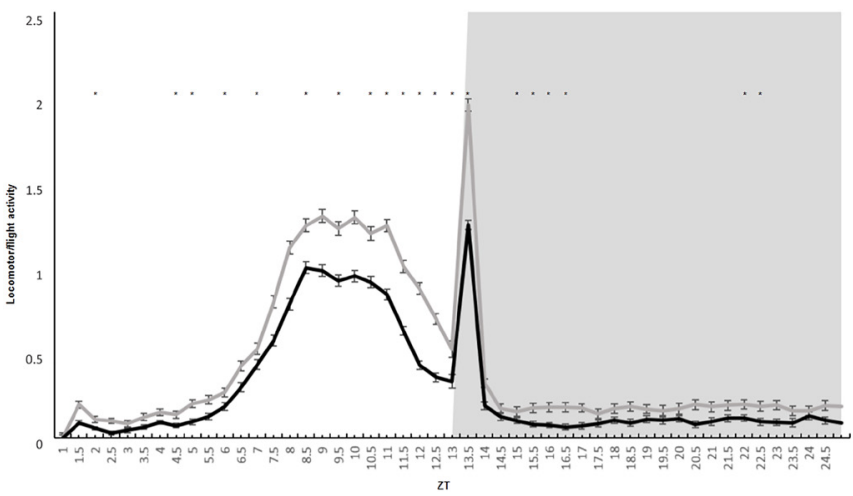

Figure 1. Average locomotor/flight activity of Aedes aegypti females infected with CHIKV ( $\mathrm{n}=144$, black line) and control females (grey line, $\mathrm{n}=139$ ) along seven days, under LD12:12. It is possible to observe a significant decrease in the locomotor flight activity of CHIKV infected females in the last hours of the light phase and in the transition between light and dark. Light area means light phase and grey area means dark phase. Error bars were shown for each $30 \mathrm{~min}$ interval. $\mathrm{X}$ axis represents the William's mean of locomotor activity and the $\mathrm{Y}$ axis represents the Zeitgeber Time. Asterisks represent the significance of the Mann-Whitney test, where $p<0.05$.
Interestingly, we observed that CHIKV infection altered the Ae. aegyti locomotor/flight behavior, where which infected females showed a significant decrease in the activity along the light phase, with a more pronounced drop during the highest peaks of activity (between ZT8 and ZT11 and ZT13.5) (MannWhitney test, where $p<0.05$; Figure 1 , black line)

\section{DISCUSSION}

Knowing that vaccines for CHIKV arbovirus are still under study and as its infection can cause debilitating symptoms, it is necessary to focus on the containment of this virus spread by controlling its $A$ e. aegypti vector ${ }^{7,15}$. For this, more studies should be carried out in order to know more the aspects of the biology and behavior of this species to improve the development of control alternatives.

Regarding the locomotor behavior and physiology of $A$ e. aegypti, recent studies have observed that these aspects could be differently modulated according to the types of arboviruses that infected the mosquitoes. For example, even though they are from the same genus (Flavivirus), DENV2 infection increased mosquito's locomotor activity while ZIKV infection caused a decreasing in its locomotor/flight activity ${ }^{10,11}$. Additionally, in relation to other features of vector physiology, it was seen that DENV2 infection decreases the number of eggs laid for females, but ZIKV does not affect this parameter ${ }^{11,12}$. Together, these interesting data show antagonistic behaviors modulated for arboviruses infection of the same genus, which led us to question how it would be the response of the vector against an arbovirus infection of a different family.

Here, we present the first study that focuses on describing the behavior of locomotion/flight of Ae. aegypti females when infected with CHIKV arbovirus. We observed a decrease in their locomotor/flight activity, similar to the effect seen when females were infected with ZIKV, despite the viruses belong to different families.

This decrease in activity itself did not negatively influence the spread of the virus in Brazil due to the very large geographical distribution and infestation rates by this mosquito recorded in the country ${ }^{6,16}$. So, although infected females move less than uninfected ones, the huge population of the CHIKV main vector in the country can ensure considerable transmission and spread in the country ${ }^{16}$. In addition to the high population density of Ae. aegypti in the Brazilian territory, at least two other factors are likely to help in compensating the lower mobility of infected females in the spread and intensity of transmission: the high vector competence of Brazilian populations to CHIKV and the short extrinsic incubation period of CHIKV (virus can be expectorated by vector within 3 days after a blood meal on a viremic individual $\left.{ }^{17,18}\right)$. As a result, a large number of infected people, reaching 47.830 reported cases in Brazil between 2014 and 2015. 
Recently, in 2019, until Entomological Week 34, the Southeast and Northeast regions had the highest values of incidence rate of probable cases, with 94.1 cases $/ 100$ thousand inhabitants and 39.3 cases/100 thousand inhabitants, respectively ${ }^{6,15,19}$. However, interestingly, the spread of CHIKV in Brazil was not as fast as expected in a country with a naive population. Despite sharing the same vector (Ae. aegypti), the spatial-temporal spread and annual number of cases due to ZIKV and CHIKV, following their invasion in Brazil was quite distinct: while ZIKV infected thousands of people and almost cover the country in one year, CHIKV inexplicably did not do so ${ }^{11,20}$. In 2014, CHIKV was firstly detected in Brazil in two states far apart: one in the north and another in the northeast. Outbreaks or clusters of cases were reported over 2014 in other four states ${ }^{5,6}$. This unexpected epidemiological profile of spread and incidence has not yet been explained. It is possible the alteration of the behavior of the CHIKV infected Ae. aegypti reported herein may have that somehow contributed to this phenomenon.

Our findings reinforce the importance of further studies focusing on analysis of the physiology and behavior of Ae. aegypti infected with CHIKV, for example, analysis of female fecundity and fertility, daily survival of the vector, aspects of hematophagy, among others. In addition, it is necessary to understand the influence of the circadian clock on this locomotor behavior of CHIKV-infected Ae. aegypti, performing experiments in constant dark conditions. Besides, it is important to investigate the effects of infection at the molecular level.

We consider that our data will support the better understanding of how the Ae. aegypti behavior is impacted by the viral infection and could highlight some important aspects of host-vector interaction, which in future may serve as a basis for control strategies of this vector mosquito.

\section{ACKNOWLEDGEMENTS}

To Maria Ignez Lima Bersot and Robson Costa da Silva for technical support; Dr. Luciana Araripe for helping with statistical analysis; João Marano for helping with the figure; We would like to dedicate this paper to Alexandre Afrânio Peixoto (in memorian), for beginning the studies on mosquito behavior and for their friendship.

\section{REFERENCES}

1. Clements A. The biology of mosquitoes: development, nutrition and reproduction. London: Chapman and Hall. 1992; P. xiv-xxii.
2. Consoli RAGB, Lourenço-de-Oliveira R. Principais mosquitos de importância sanitária no Brasil. Rio de Janeiro: Fiocruz; 1994; P. 115 117.

3. Bruno RV, Farnesi LC, Araripe LO. Current Topics in the Epidemiology of Vector-Borne Diseases. The Effects of Infection on Mosquito Rhythmic Behavior; 2019; P. 1-16.

4. Ross RW. The Newala Epidemic, III. The virus: isolation, pathogenic properties and relationship to the epidemic. J. Hyg (London).1956; 54(2): $177-191$.

5. Nunes MR, Faria NR, de Vasconcelos JM, Golding N, Kraemer MU, de Oliveira LF, et al. Emergence and potential for spread of Chikungunya virus in Brazil. BMC Med. 2015; 13:102.

6. Honório NA, Câmara DC, Calvet GA, Brasil P. Chikungunya: an arbovirus infection in the process of establishment and expansion in Brazil. Cad. Saúde Pública. 2015; 31(5):906-8.

7. Tharmarajah K, Mahalingam S, Zaid A. Chikungunya: vaccines and therapeutics. F1000 Res. 2017; 6:2114.

8. Clements AN. The biology of mosquitoes: sensory reception and behavior. Vol. II. Wallingford: CABI Publishing; 1999; P. 206-230.

9. Gentile C, Rivas GB, Meireles-Filho AC, Lima JB, Peixoto AA. Circadian expression of clock genes in two mosquito disease vectors: cry 2 is different. J Biol Rhythms. 2009; 24(6): 444-51.

10. Lima-Camara TN, Bruno RV, Luz PM, Castro MG, Lourenço-de-Oliveira $\mathrm{R}$, Sorgine $\mathrm{MH}$, et al. Dengue infection increases the locomotor activity of Aedes aegypti females. PLoS One. 2011; 6(3): e17690.

11. Padilha KP, Resck MEB, Cunha OATD, Teles-de-Freitas R, Campos SS, Sorgine HF, et al. Zika infection decreases Aedes aegypti locomotor activity but does not influence egg production or viability. Mem Inst Oswaldo Cruz. 2018; 113. e180290.

12. Maciel-de-Freitas R, Koella JC, Lourenço-de-Oliveira R. Lower survival rate, longevity and fecundity of Aedes aegypti (Diptera: Culicidae) females orally challenged with dengue virus serotype 2 . Trans R Soc Trop Med Hyg. 2011; 105(8): 452-8.

13. Oliveira JH, Talyuli OA, Goncalves RL, Paiva-Silva GO, Sorgine MH, Alvarenga PH, et al. Catalase protects Aedes aegypti from oxidative stress and increases midgut infection prevalence of Dengue but not Zika. PLoS Negl Trop Dis. 2017; 11(4): e0005525.

14. Williams C. The use of logarithms in the interpretation of certain entomological problems. Ann Appl Biol. 1937; 24:404-14.

15. Teixeira MG, Andrade AM, Costa Mda C, Castro JN, Oliveira FL, Goes $\mathrm{CS}$, et al. East/Central/South African Genotype Chikungunya Virus, Brazil, 2014. Emerg Infect Dis. 2015; 21(5):906-7.

16. Maciel-de-Freitas R, Eiras ÁE, Lourenço-de-Oliveira R. Calculating the survival rate and estimated population density of gravid Aedes aegypti (Diptera, Culicidae) in Rio de Janeiro, Brazil. Cad. Saúde Pública. 2008; 24(12): 2747-54

17. Vega-Rúa A, Zouache K, Girod R, Failloux AB, Lourenço-de-Oliveira R. High level of vector competence of Aedes aegypti and Aedes albopictus from ten American countries as a crucial factor in the spread of Chikungunya virus. J Virol. 2014; 88(11):6294-306.

18. Vega-Rúa A, Lourenço-de-Oliveira R, Mousson L, Vazeille M, Fuchs S, Yébakima A, et al. Chikungunya virus transmission potential by local Aedes mosquitoes in the Americas and Europe. PLoS Negl Trop Dis. 2015; 20;9(5):e0003780.

19. Secretaria de Vigilância em Saúde, Ministério da Saúde. Monitoramento dos casos de arboviroses urbanas transmitidas pelo Aedes (dengue, chikungunya e Zika). Semanas Epidemiológicas 1 a 34; 2019. http:// portalarquivos2.saude.gov.br/images/pdf/2019/setembro/11/BEarbovirose-22.pdf (acessado em 16/jan/2020).

20. Secretaria de Vigilância em Saúde, Ministério da Saúde. Situação epidemiológica da infecção pelo vírus Zika no Brasil, de 2015 a 2017. 2018. http://portalarquivos2.saude.gov.br/images/pdf/2018/ novembro/12/2018-034.pdf (acessado em 16/jan/2020). 


\section{Sleep and maternal behavior in the postpartum rat after haloperidol and midazolam treatments}

Florencia Peña ${ }^{1}$

Mayda Rivas ${ }^{1}$

Joaquín Gonzalez ${ }^{1}$

Natalia Schwarzkopf ${ }^{1}$

Pablo Torterolo ${ }^{1}$

Annabel Ferreira ${ }^{2}$

Luciana Benedetto ${ }^{1^{*}}$

${ }^{1}$ Departamento de Fisiología, Facultad de Medicina, Universidad de la República, Montevideo, Uruguay. ${ }^{2}$ Sección de Fisiología y Nutrición, Facultad de Ciencias, Universidad de la República, Montevideo, Uruguay.

\begin{abstract}
The postpartum period is a stage in the female's life during which the incidence of emotional and psychiatric disorders is increased. Antipsychotic drugs such as Haloperidol (HAL), as well as anxiolytic agents such as Midazolam (MID), can be prescribed during this period. However, their effects on maternal behavior and sleep are scarcely known. Therefore, we aimed to determine the effects of MID and HAL on maternal behavior and sleep of postpartum rats. For this purpose, mother rats were implanted for polysomnographic recording and treated either with HAL (0.4 $\mathrm{mg} / \mathrm{kg}$, i.p.), MID (6.0 mg/kg, i.p.) or its corresponding vehicles. Maternal behavior and sleepwakefulness states were recorded during 4 hours immediately after the reunion of the pups. HALtreated mothers showed a decrease in the number of pups' retrievals as well as deficits in the reunion of the pups into the nest. In addition, the nursing time, the duration of nursing episodes as well as the litter weight gain (LWG) increased after HAL treatment compared to control values. Compared to the control values, HAL increased the time spent in slow wave sleep (SWS) and intermediate stage (IS) without provoking changes in REM sleep. On the other hand, rats treated with MID showed deficits to reunite the entire litter into the nest. Also, MID-treated mothers showed a reduction in the number of nursing episodes and an increase in their duration, with no changes in LWG compared to control treatment. Sleep analysis revealed that MID decreased the time spent in light sleep, increased the time spent in SWS, while IS and REM parameters remained unchanged. Our findings show that both drugs affected maternal behavior and sleep, each one in a specific pattern. In addition, while most sleep characteristics resemble the ones described for males, some of the differences found could rely on the unique profile of the postpartum female, underlying the necessity to deepen the studies of the effects of drugs in this particular period.
\end{abstract}

Keywords: Benzodiazepine; Nursing; Antipsychotic; Sleep; Maternal Behavior
Corresponding author:
Luciana Benedetto
E-mail: lbenedet@fmed.edu.uy

DOI: $10.5935 / 1984-0063.20200019$ 


\section{INTRODUCTION}

The early postpartum period has been characterized as a time in women' life of particular susceptibility to emotional and psychiatric disorders, which can adversely affect the health and wellbeing of new mothers, their infants and their families. For instance, women experience 22 times more psychotic or mania episodes in the postpartum period than in any other period of their lives ${ }^{1}$. In addition, the prevalence of postpartum anxiety is high during the postpartum period, ranging from 13 to $40 \%$ depending on the type of anxiety assessment and the scale used, among other factors ${ }^{2}$.

Drugs, such as the D1/D2 antagonist Haloperidol (HAL) and the GAB-A benzodiazepine agonist Midazolam (MID) ${ }^{3,4}$ have been extensively prescribed for the treatment of psychotic episodes, mania, anxiety and sleep disorders ${ }^{5}$. Different studies show that benzodiazepines are compatible with breastfeeding ${ }^{6}$ and assigned as "good safety profile" based on the plasma/ milk ratio with very low effects on the infant ${ }^{7}$. In the same way, antipsychotic drugs, such as HAL, are also used during lactation in women with bipolar disorder or schizophrenic disease. A standing problem of these drugs are daytime drowsiness and motor and cognitive skills disruptions ${ }^{8,9}$ that could lead to deleterious maternal behavior or increase probability of accidents of the mother when interacting with the newborn.

The effects of HAL and MID on sleep have been extensively studied in male rats, but studies in females, particularly during the postpartum period, are scarce or null. Interestingly, we showed that the effects of anxiolytic drugs differ between virgin and maternal rats, stressing the uniqueness of the female physiology during the postpartum period and the necessity to deepen the study of the interaction between drugs and the particular physiological changes of this period ${ }^{10,11}$.

Therefore, the purpose of the study was to determine the effects of two drugs, commonly used in clinical practice: HAL and MID, on the maternal behavior and sleep of postpartum rats. To this end we performed polysomnographic recordings as well as a maternal behavior analyses during four hours after the systemic treatment with HAL and MID of postpartum rats.

\section{METHODS}

\section{Animals and housing}

Sixteen primiparous Wistar female rats (260-310 g) and their pups were used in this study. All animal use and experimental procedures were in strict accordance with the "Guide to the care and use of laboratory animals" (8th edition, National Academy Press, Washington D.C., 2011) and approved by the Institutional Animal Care Committee. All efforts were made to minimize the number of animals used and their suffering.

Two days before giving birth, pregnant females were housed individually in transparent cages $(40 \times 30 \times 20 \mathrm{~cm})$ containing shredded paper towels as nest-building material.
The animals were placed in a temperature-controlled $\left(22 \pm 1{ }^{\circ} \mathrm{C}\right)$, sound-proof and electromagnetically shielded recording chamber fitted with slip rings and cable connectors for bioelectrical recordings, under a 12-h light/dark cycle (lights on at 6:00 a.m.), with ad libitum access to food and water. On postpartum day 1 (PPD1, birth = day 0), litters were culled to four female and four male pups per mother.

\section{Stereotaxic surgery}

The surgical procedures were similar as the performed in previous studies of our group ${ }^{12}$. On the morning of PPD1, females were anesthetized with a mixture of ketamine/ xylazine/acepromazine maleate $(80 / 2.8 / 2.0 \mathrm{mg} / \mathrm{kg}$, i.p.). Using a stereotaxic device, female rats were implanted with cortical electroencephalogram (EEG) electrodes and dorsal neck muscle electromyogram (EMG) electrodes for the assessment of sleep and wakefulness (W) states. Recording electrodes for EEG were placed in the frontal cortex (AP $=+3.0 ; \mathrm{ML}=2.0)$, parietal cortex $(\mathrm{AP}=-4.0, \mathrm{ML}=3.0)$, occipital cortex (AP $=-7.0, \mathrm{ML}=3.0)$, and an electrode was placed over the cerebellum as a reference $(\mathrm{AP}=-11.0$, ML $=0.0)^{13}$. Two additional stainless-steel screws were implanted into the skull as anchors. All electrodes were soldered to a sixpin connector and cemented to the skull using dental acrylic.

\section{Experimental design}

All experiments were performed between PPD6-8 during the light phase of the cycle. A baseline recording was performed the day before the beginning of the experimental sessions to corroborate that all sleep parameters and maternal behaviors were adequate. Sixteen rats were assigned randomly to one of two independent groups: HAL $(\mathrm{n}=8)$ or MID $(\mathrm{n}=8)$. Within each group, each animal received two i.p. injections on PPD6 and PPD8: 1. HAL $(0.4 \mathrm{mg} / \mathrm{kg})$ and vehicle (distilled water), or 2 . MID $(6.0 \mathrm{mg} / \mathrm{kg})$ and vehicle (distilled water) in a counterbalanced design in such a way that half of the animals in each group received a drug injection on PPD6 and a vehicle injection on PPD8, while the other half received the opposite protocol injection. No drug was administered in the day between experimental sessions.

\section{Drugs}

The drugs used were HAL $(0.4 \mathrm{mg} / \mathrm{kg}$ i.p., Fármaco Uruguayo, Uruguay) and MID $(6.0 \mathrm{mg} / \mathrm{kg}$ ip, Laboratorio Roche, Uruguay). We selected HAL dose considering that most reductions in maternal behavior parameters were affected by this dose ${ }^{14}$. In addition, sleep enhancement is observed from 0.2 up to $3.0 \mathrm{mg} / \mathrm{kg} \mathrm{HAL}$ in male rats ${ }^{15-18}$. The dose of MID was selected because it represent an intermediate dose among those reported in several studies of sleep parameters in male rats, that vary from 3 to $10 \mathrm{mg} / \mathrm{kg}^{19-21}$. As far as we know, there are no studies that used MID in lactating rats. 


\section{Experimental sessions}

During each experimental day, pups were removed from the maternal cage and placed under a heat lamp at 9 a.m. for four hours. One hour (for HAL) or fifteen minutes (for MID) before the reunion with the pups, each female rat was injected with the drug or vehicle, returned to the home cage and connected to the recording system for posterior polysomnographic recording (protocol adapted from $^{22}$; because MID effects where already evident a few minutes after injection, latency was shortened to 15 minutes). When maternal separation was completed, the entire litter was weighed and scattered in the maternal cage opposite to the nest and the polysomnographic and maternal behavior recordings were initiated. After four-hour-session, each mother rat was disconnected from the recording device and the entire litter was weighed again $\left(\right.$ adapted from $\left.{ }^{23}\right)$. This procedure was repeated twice in each rat, once with vehicle and the other with the corresponding drug.

\section{Maternal behavior}

The number of retrievals of the pups into the nest and the latency to group the entire litter were measured. If the mother did not retrieve a pup into the nest after five min after placing the pups in the cage, the pup was placed in the nest by the researcher and the latency to reunite the entire litter was assigned as 300 seconds $^{24}$.

Latency to the adoption of a nursing posture and the total duration of these postures were measured. The total nursing time was calculated for the 4-h-recording session and for each hour separately from digital videos captured using a video tape recording attached to the Spike 2 software (CED, Cambridge, UK). Nursing postures included kyphotic and supine ones (adapted from ${ }^{12,25}$ ). In addition, the number of milk ejections was quantified indirectly through the stretching behavior of the pups ${ }^{26,27}$. The litter weight gain (LWG) was used as an indirect measurement of the amount of ejected milk ${ }^{14,28}$ and was calculated as the percentage of the difference between the final and initial weight of the entire litter.

\section{Sleep recording}

Bioelectric signals were amplified $(\times 1000)$, filtered (0.1-500 Hz), sampled (1024 Hz, 16 bits) and stored in a PC for further analysis using the Spike 2 software. The states of light sleep (LS), slow wave sleep (SWS), intermediate stage (IS, transition from SWS to REM sleep), REM sleep and W were determined in 5-s epochs with standard criteria ${ }^{28,29}$.

Total time spent in W, LS, SWS, no-REM sleep (NREM, LS + SWS), IS and REM sleep over the total recording time and each hour separately were analyzed. In addition, sleep latencies (first episodes $\geq 20 \mathrm{~s}$ from the beginning of the recordings), number, and duration of episodes of each state were calculated.

\section{Statistics}

Data from maternal parameters did not follow a normal distribution (Kolmogorov-Smirnov test, $\mathrm{p}<0.05$ ). Thus, maternal parameters are presented as median \pm SIQR (semi-interquartile range) and statistical differences between experimental and control groups were evaluated using a Wilcoxon test for paired samples ${ }^{30}$. As sleep data follow a normal distribution (Kolmogorov-Smirnov test, $\mathrm{p}>0.05$ ), values are presented as mean \pm S.E.M. (standard error) and comparisons between experimental and control groups were performed by means of the Student t-test for dependent samples. The criterion used to discard the null hypotheses in all cases was $\mathrm{p}<0.05$.

\section{RESULTS}

\section{Effects of i.p. injection of HAL}

\section{Maternal Behavior}

The results of the effects of HAL on maternal behavior are shown in Table 1 . The number of pups' retrievings significantly decreased and the latency to group the entire into the nest significantly increased after HAL treatment compared to control treatment.

Table 1. Effects of i.p. injections of haloperidol (HAL) on maternal behavior parameters during 4-hour sessions.

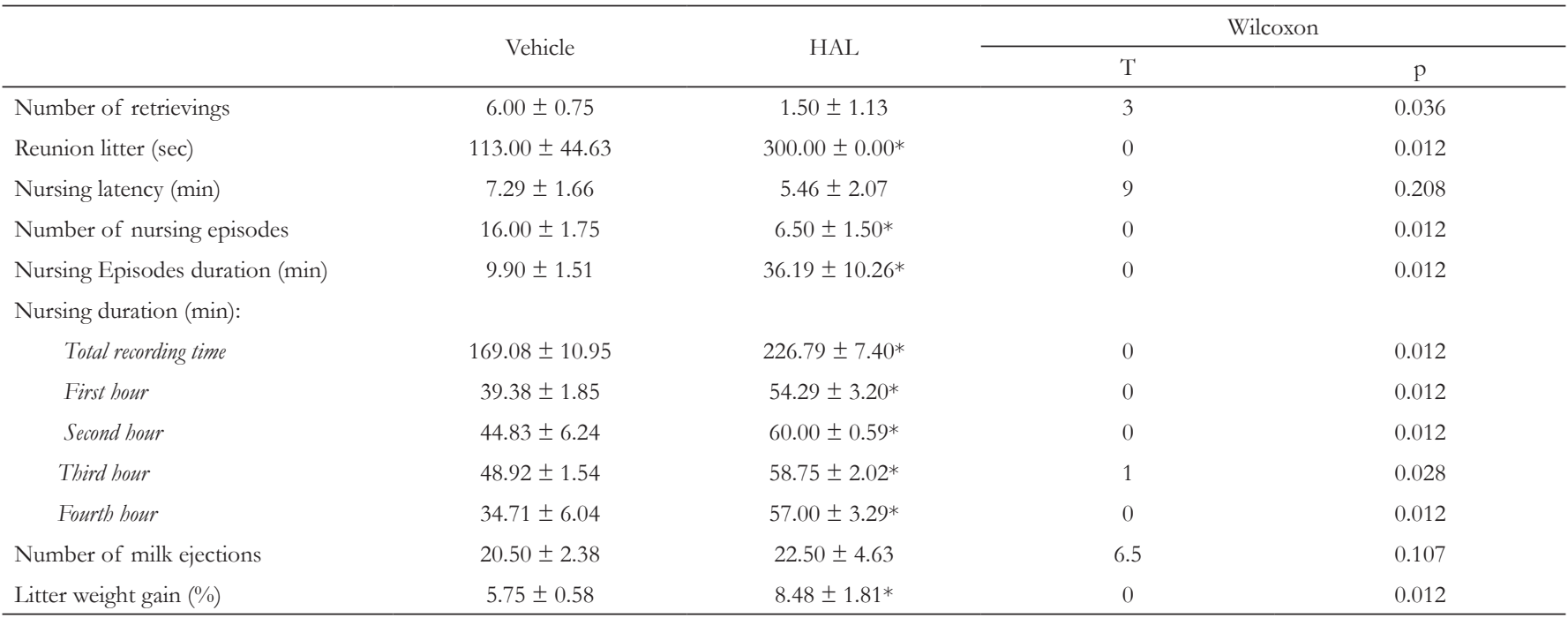

Data are presented as median \pm semi-interquartile range of eight rats. Significant differences were evaluated using a Wilcoxon test for paired samples and indicate by asterisks. 
In addition, while all rats treated with vehicle were able to group the entire litter into the nest within the five initial minutes after the reunion with the pups, none of the eight rats treated with HAL could group the entire litter into the nest within the five minutes after reunion.

Furthermore, HAL treatment produced a significant increase in females' nursing time compared to that of females treated with vehicle; this enhancement was observed both in the total recording time and in each hour independently (Table 1). Besides, HAL-treated mothers exhibited a reduced number of nursing bouts with longer duration compared to that of vehicle treated mothers, while the latency to begin nursing did not differ between groups (Table 1). In accordance, LWG increased in HAL group compared to that of control group, but the number of milk ejections did not vary between groups (Table 1).

\section{Sleep and waking states}

The effects of HAL on sleep parameters are shown in Table 2 and Figure 1. HAL treated females showed a significant reduction in the time spent in $\mathrm{W}$ compared to that of the vehicle treated group, both in the total recoding time and in each hour separately. Also, HAL produced an increase in the number but a decrease in the duration of $\mathrm{W}$ episodes compared to control values (Table 2 ).
Moreover, HAL treatment produced similar modifications in LS and SWS. Specifically, HAL-treated mothers spent more time in these two stages, both in the total recording time and the first two hours when analyzed independently (Table 2 and Figure 1). In addition, the number of LS and SWS episodes was significantly increased compared to that of control mothers but their duration remained unchanged (Table 2). The latency to NREM sleep decreased after HAL treatment compared to that of the control group (Table 2).

The time mothers spent in IS increased after HAL compared to that of vehicle treatment, both in the entire recording session and in each hour individually, except for the third hour (see Table 2 and Figure 1); this effect was at the expense of a significant increase in the number of episodes (Table 2).

None of the REM sleep parameters studied varied between HAL and vehicle treatment (Table 2 and Figure 1).

\section{Effects of i.p. injection of MID}

\section{Maternal Behavior}

Table 3 shows the results of MID on maternal behavior. The number of retrievings did not vary between groups, most MID-treated mothers (six out of eight) were unable to group the entire litter into the nest within the first five minutes after the reunion of the pups, while all control mothers grouped the entire litter within this time.

Table 2. Effects of i.p. injections of haloperidol (HAL) on sleep and waking parameters during 4-hour sessions.

\begin{tabular}{|c|c|c|c|c|}
\hline & \multirow{2}{*}{ Vehicle } & \multirow{2}{*}{ HAL } & \multicolumn{2}{|c|}{ T-Test } \\
\hline & & & $\mathrm{t}$ & $\mathrm{p}$ \\
\hline \multicolumn{5}{|l|}{ Wakefulness } \\
\hline Number of episodes & $130.25 \pm 4.91$ & $168.38 \pm 11.31 *$ & 2.908 & 0.023 \\
\hline Episodes duration (min) & $0.78 \pm 0.07$ & $0.35 \pm 0.04 *$ & 9.044 & 0.000 \\
\hline Number of episodes & $171.63 \pm 9.03$ & $240.75 \pm 18.73^{*}$ & 3.206 & 0.015 \\
\hline Episodes duration (min) & $0.14 \pm 0.01$ & $0.14 \pm 0.01$ & 0.621 & 0.554 \\
\hline \multicolumn{5}{|l|}{ Slow Wave Sleep } \\
\hline Total duration (min) & $90.84 \pm 8.31$ & $114.64 \pm 8.90 *$ & 3.528 & 0.010 \\
\hline Number of episodes & $137.38 \pm 6.71$ & $210.13 \pm 14.63^{*}$ & 4.641 & 0.002 \\
\hline Number of episodes & $26.63 \pm 2.99$ & $50.38 \pm 7.35^{*}$ & 3.082 & 0.018 \\
\hline Episodes duration (min) & $0.33 \pm 0.02$ & $0.33 \pm 0.05$ & 0.053 & 0.960 \\
\hline \multicolumn{5}{|l|}{ REM Sleep } \\
\hline Total duration (min) & $16.51 \pm 1.97$ & $16.70 \pm 1.93$ & 0.055 & 0.958 \\
\hline Number of episodes & $17.63 \pm 2.25$ & $26.63 \pm 4.34$ & 1.611 & 0.151 \\
\hline Episodes duration (min) & $0.97 \pm 0.09$ & $0.68 \pm 0.08$ & 2.293 & 0.056 \\
\hline Total NREM duration (min) & $114,15 \pm 22,88$ & $147,20 \pm 23.66^{*}$ & 6,474 & 0.000 \\
\hline Latency REM (min) & $57.44 \pm 10.16$ & $57.21 \pm 6.09$ & 0.016 & 0.987 \\
\hline Latency NREM (min) & $9.14 \pm 1.75$ & $4.91 \pm 0.70^{*}$ & 2.979 & 0.021 \\
\hline
\end{tabular}

Data are presented as mean \pm standard error of eight rats. Significant differences were evaluated using a t-test for paired samples and indicate by asterisks. 
Wakefulness

Wakefulness
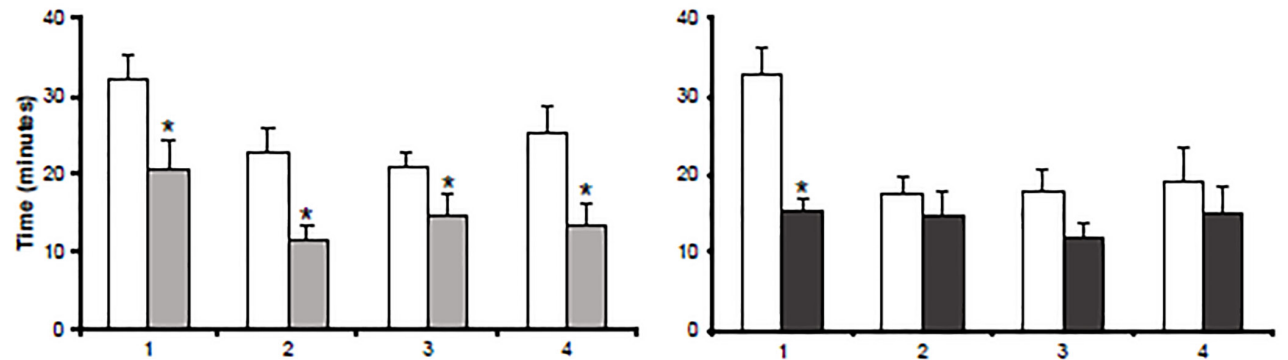

Light Sleep

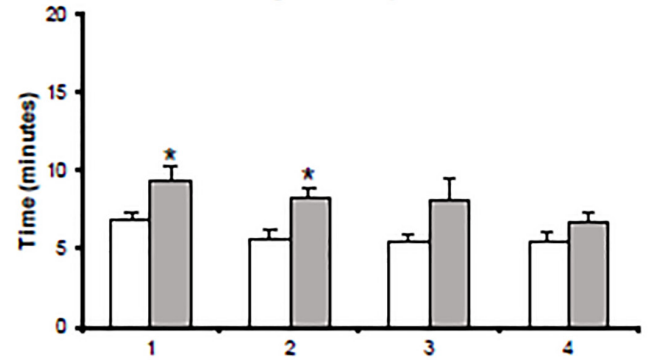

Light Sleep

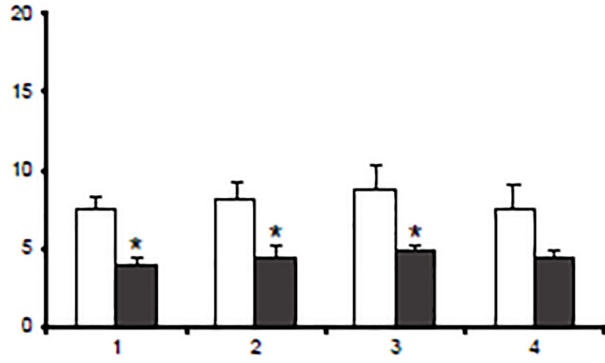

Slow Wave Sleep

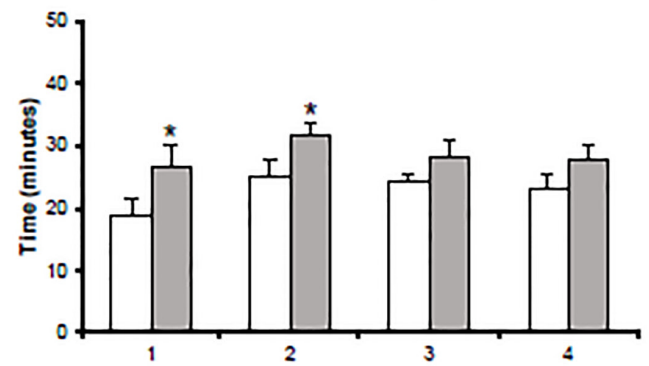

Slow Wave Sleep

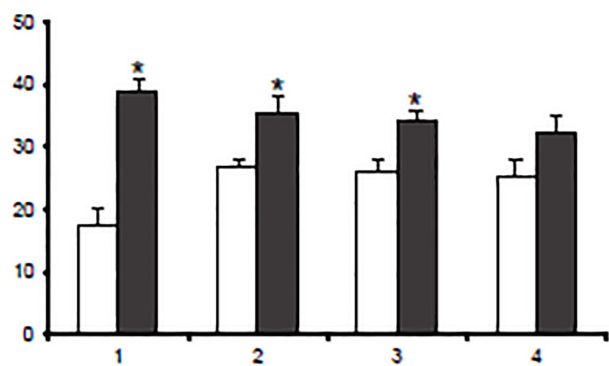

Intermediate Stage

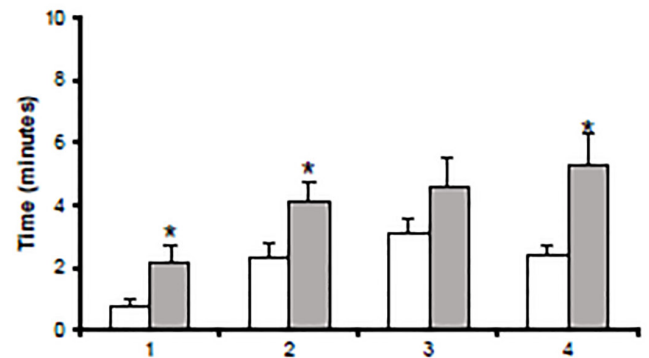

Intermediate Stage

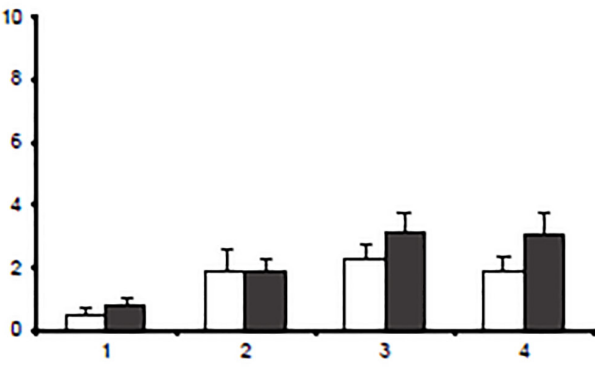

REM

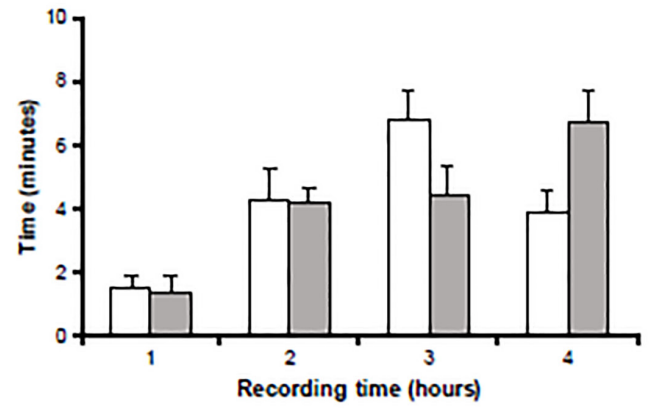

REM

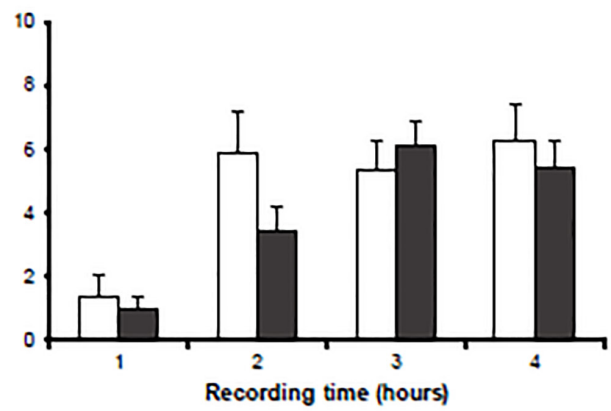

$\square$ HAL $\square$ Vehicle $\square$ MID

Figure 1. Effects of haloperidol and midazolam on sleep and waking states. Graphic charts show the mean time spent in wakefulness, light sleep, slow wave sleep, intermediate stage and REM sleep after administration i.p. of haloperidol (HAL, $0.4 \mathrm{mg} / \mathrm{kg}$ ), midazolam (MID, $6.0 \mathrm{mg} / \mathrm{kg}$ ) or its corresponding vehicles during each hour of the total recording time. Differences were determined by means of the Student $t$-test for paired samples. Asterisks $\left(^{*}\right)$ indicate significant differences compared to control values $(\mathrm{p}<0.05)$. 
In addition, nursing episodes of MID-treated mothers were fewer but longer when compared to those of the vehicle treated group. MID treatment did not provoke additional significant changes in other maternal behaviors analyzed (see Table 3).

\section{Sleep and waking states}

The total time spent in $\mathrm{W}$ as well as the time spent during the first hour was significantly reduced in the MID treated group compared to those of vehicle group. Also, the number of $\mathrm{W}$ episodes decreased in the MID group when compared to control group (Table 4).

Table 3. Effects of i.p. injections of midazolam (MID) on maternal behavior parameters during 4-hour sessions.

\begin{tabular}{|c|c|c|c|c|}
\hline & \multirow{2}{*}{ Vehicle } & \multirow{2}{*}{ MID } & \multicolumn{2}{|c|}{ Wilcoxon } \\
\hline & & & $\mathrm{T}$ & $\mathrm{p}$ \\
\hline Number of retrievings & $6.50 \pm 0.50$ & $3.50 \pm 2.88$ & 9 & 0.208 \\
\hline Reunion litter (sec) & $88.00 \pm 31.00$ & $300.00 \pm 7.63^{*}$ & 1 & 0.017 \\
\hline Nursing latency (min) & $66.00 \pm 41.75$ & $63.00 \pm 16.25$ & 16 & 0.779 \\
\hline Number of nursing episodes & $16.00 \pm 3.50$ & $6.50 \pm 0.88^{*}$ & 0 & 0.012 \\
\hline Nursing Episodes duration (min) & $12.14 \pm 1.45$ & $28.86 \pm 4.03^{*}$ & 1 & 0.017 \\
\hline \multicolumn{5}{|l|}{ Nursing duration (min): } \\
\hline Total recording time & $179.04 \pm 20.88$ & $207.04 \pm 19.51$ & 16 & 0.779 \\
\hline First hour & $41.00 \pm 5.16$ & $48.50 \pm 4.13$ & 9 & 0.208 \\
\hline Second hour & $50.58 \pm 6.70$ & $56.25 \pm 3.18$ & 10 & 0.499 \\
\hline Third hour & $50.54 \pm 6.27$ & $57.25 \pm 6.67$ & 13 & 0.866 \\
\hline Fourth hour & $48.96 \pm 13.52$ & $46.50 \pm 8.64$ & 17 & 0.889 \\
\hline Number of milk ejections & $19.00 \pm 2.13$ & $20.50 \pm 3.75$ & 16 & 0.779 \\
\hline Litter weight gain $(\%)$ & $6.50 \pm 0.22$ & $4.66 \pm 0.22$ & 8 & 0.161 \\
\hline
\end{tabular}

Data are presented as median \pm semi-interquartile range of eight rats. Significant differences were evaluated using a Wilcoxon test for paired samples and indicate by asterisks.

Table 4. Effects of i.p. injections of midazolam (MID) on sleep and waking parameters during 4-hour sessions.

\begin{tabular}{|c|c|c|c|c|}
\hline & \multirow{2}{*}{ Vehicle } & \multirow{2}{*}{ MID } & \multicolumn{2}{|c|}{ T-Test } \\
\hline & & & $\mathrm{t}$ & $\mathrm{p}$ \\
\hline \multicolumn{5}{|l|}{ Wakefulness } \\
\hline Number of episodes & $139.63 \pm 8.13$ & $110.13 \pm 10.16^{*}$ & 3.225 & 0.015 \\
\hline Episodes duration (min) & $0.63 \pm 0.05$ & $0.54 \pm 0.06$ & 1.572 & 0.160 \\
\hline \multicolumn{5}{|l|}{ Light Sleep (LS) } \\
\hline Number of episodes & $198.13 \pm 16.18$ & $133.50 \pm 12.73^{*}$ & 4.501 & 0.003 \\
\hline Episodes duration (min) & $0.16 \pm 0.01$ & $0.13 \pm 0.00$ & 1.984 & 0.088 \\
\hline \multicolumn{5}{|l|}{ Slow Wave Sleep (SWS) } \\
\hline Total duration (min) & $95.23 \pm 6.35$ & $141.00 \pm 5.21 *$ & 7.132 & 0.000 \\
\hline Number of episodes & $156.88 \pm 10.23$ & $129.13 \pm 13.36$ & 1.693 & 0.134 \\
\hline Number of episodes & $24.63 \pm 3.42$ & $28.88 \pm 5.97$ & 0.835 & 0.431 \\
\hline Episodes duration (min) & $0.25 \pm 0.02$ & $0.32 \pm 0.03$ & 2.194 & 0.064 \\
\hline \multicolumn{5}{|l|}{ REM Sleep } \\
\hline Total duration (min) & $18.96 \pm 2.26$ & $15.99 \pm 1.52$ & 1.557 & 0.163 \\
\hline Number of episodes & $19.75 \pm 2.12$ & $21.50 \pm 3.96$ & 0.566 & 0.589 \\
\hline Episodes duration (min) & $0.98 \pm 0.12$ & $0.98 \pm 0.23$ & 0.020 & 0.985 \\
\hline Total NREM duration (min) & $127.24 \pm 14.54$ & $158.53 \pm 13.98^{*}$ & 5,041 & 0.001 \\
\hline Latency REM (min) & $54.66 \pm 4.50$ & $68.74 \pm 13.70$ & 1.223 & 0.261 \\
\hline Latency NREM (min) & $8.70 \pm 0.97$ & $4.09 \pm 0.69 *$ & 3.136 & 0.016 \\
\hline
\end{tabular}

Data are presented as mean \pm standard error of eight rats. Significant differences were evaluated using a $\mathrm{t}$-test for paired samples and indicate by asterisks. 
MID-treated mothers spent significantly less time in LS during the total recording time and during the first three hours individually (see Table 4, Figure 1). Also, the number of LS episodes decreased after MID treatment compared to control group.

The time spent in SWS in the entire recording session and during the first three hours individually was increased after MID compared to vehicle injection (Table 4 and Figure 1). In addition, the duration of episodes was significantly increased after MID treatment. The latency to NREM sleep decreased with MID treatment (see Table 4).

Sleep parameters of IS and REM did not differ between groups (Table 4 and Figure 1).

\section{DISCUSSION}

In the present study, we showed that the systemic administration of two drugs, HAL and MID, commonly used in clinical practice, affected the maternal behavior and sleep parameters of postpartum female rats in a specific way. In addition, we described for the first time the effects of HAL on the transitional stage to REM sleep, showing that a significant increase was observed.

\section{Effect of HAL on Maternal Behavior}

HAL-treated mothers were unable to reunite the entire litter into the nest and, consequently, the latency to retrieve the entire litter increased compared to that of vehicle treated mothers. In addition, the number of retrievals displayed by HAL-treated mothers significantly decreased. These effects are similar to those previously reported ${ }^{22,24,31}$, showing that HAL disrupts most active components of maternal behavior. It could be argued that these deficits are related to motor impairment provoked by HAL ${ }^{32-34}$. However, Giordano et al. (1990) show that postpartum females treated with HAL, which were not able to reunite the entire litter into the nest, could transport food, suggesting that the drug may specifically affect maternal behavior $^{24}$. In the same line, Li (2015) found that non-cataleptic doses of HAL produce deficits in active maternal behavior suggesting that the effects of dopaminergic antagonists would be mainly motivational ${ }^{35}$. In same sense, Zaho et al. (2009) postulate that the sedative effects of these drugs would not be the underlying cause of the reduction in the active components of maternal behavior but probably a reduction in motivation ${ }^{36}$.

Several studies show that HAL, acting in the mesocorticolimbic dopamine (DA) system, interferes with the motivational aspects of maternal behavior. Particularly, both the systemic administration of DA receptor antagonists ${ }^{22,24,35}$ as well as the micro-injection of DA receptor antagonists into the nucleus accumbens ${ }^{37,38}$ disrupt most forms of active maternal behaviors, such as retrieval and grouping of the pups at the nest site, pup licking and nest building. Also, it has been reported that the interaction of the mother with the pups is associated with increases in DA release $\mathrm{e}^{39}$ and $\mathrm{cFos}$ expression in the nucleus accumbens of the mothers ${ }^{40,41}$.
Together with a reduction in active maternal behavior, we found an increase in the total nursing time after HAL treatment. Interestingly, previous studies have shown that the interference with the DA system only affect the active maternal behavior while leaving nursing, a motorically inactive behavior, relatively intact or even promoting $\mathrm{it}^{14,24}$. Even if most evidences show that HAL interferes with maternal motivation, in the present study we observed that HAL promoted NREM sleep. This somnogenic effect of HAL could account, at least in part, for the prolonged time in nursing positions of mother rats.

We also observed that the LWG, an indirect measure of the amount of milk ejected ${ }^{28,42}$, increased after HAL treatment, as previously described by Stern $(1991)^{14}$. However, the number of pups' stretching responses, an indirect measure of the number of milk ejections ${ }^{26}$, did not differ between groups. It is well established that HAL produces an increase in serum prolactin ${ }^{43-46}$ and, therefore, in the amount of milk produced ${ }^{47}$. Hence, we can speculate that HAL induced an increase in the synthesis of milk produced, thus enhancing the total milk ejected during the 4-hour-recording session. However, we cannot discard that other factors can influence final weight litter. For instance, it has been reported that mother rats treated with HAL showed a reduction in ano-genital licking ${ }^{31}$ probably decreasing pups' urination and defecation which in turn, could affect litter weight.

\section{Effect of HAL on sleep and waking states}

The systemic injection of HAL produced a decrease in $\mathrm{W}$, an effect that has already been evidenced in previous studies in male rats ${ }^{16,18}$. In addition, this reduction of $\mathrm{W}$ was characterized by shorter but more frequent $\mathrm{W}$ episodes compared to those of control group. In contrast, Monti (1968) observed in cats that HAL $(4 \mathrm{mg} / \mathrm{kg})$ decreases the number of W episodes ${ }^{48}$. A putative explanation to this opposite effect could rely on the unique sleep profile of lactating females, which show a higher number of awakenings throughout the sleep-wake cycle than males ${ }^{49}$. These micro-arousals, possibly caused by milk ejections ${ }^{50}$ or by the interaction with the pups ${ }^{12}$, could have been intensified after HAL treatment.

We observed that the total time in both LS and SWS was longer in HAL-treated mothers compared to control rats. The promotion of SWS after HAL treatment has already been evidenced in male rats, but no LS alteration was reported ${ }^{16,18}$. Again, the particular sleep pattern observed in lactating females could account for this difference.

Possible mechanisms by which HAL promotes SWS could rely on the inhibition of the dopaminergic circuits of the ascending activating reticular system ${ }^{51}$ or on the increase of prolactin release, a sleep-promoting hormone ${ }^{52,53}$. There are anatomic and functional changes in critical areas for sleep generation in lactating females ${ }^{5-57}$ which could explain the differences between present results and male studies. 


\section{Effect of MID on Maternal Behavior}

We show that most MID-treated mothers were unable to group the entire litter into the nest within the first five minutes after the reunion of the pups compared to the control group, but the number of retrievals were unaffected. In this sense, Ferreira et al. (2000) previously showed that diazepam, also a benzodiazepine receptor agonist, reduced active maternal components (however, also $\mathrm{se}^{59}$ ). In this study, the inhibition of some components o the maternal behavior after benzodiazepines cannot $\mathrm{b}$ explained on the basis of motor impairments because maternal inhibitory effects were found without motor disturbances ${ }^{58}$.

In present study, we also showed that MID-treated and control mothers invested similar time in nursing the pups. However, the distribution of this time was different. Particularly, the nursing episodes displayed by MID-treated mothers were fewer but longer than those of control mothers. It could be suggested that the somnogenic effect of MID could lead to the delay to reunite the entire litter into the nest and also contribute to promote more consolidated nursing episodes.

\section{Effect of MID on sleep and waking states}

The systemic administration of MID produced a reduction in $\mathrm{W}$ time. This effect was similar to that described for male rats ${ }^{19,20}$. In this sense, Depoortere et al. (1995) evaluated MID $(10 \mathrm{mg} / \mathrm{kg})$ effect during the dark phase of the light-dark cycle and found a W reduction in the third hour with no reduction of $\mathrm{W}$ over the total recording time ${ }^{20}$. We found similar effects during the light phase with a slighter dose, but changes were only detected in the first hour of recording.

In addition, MID produced an increase in the total duration of NREM sleep. Specifically, the time spent in SWS was increased at the expense of an increase of the duration of its episodes. On the contrary, the time spent in LS was reduced compared to control values with a reduction both in the duration and in the number of its episodes. Previous works in male rats reported an increase in NREM sleep ${ }^{20,21}$. However, these studies did not sub-classify NREM into SWS and LS. In addition, ${ }^{19}$ distinguishes SWS from LS showing no differences in SWS but increases in LS after MID treatment in male rats. However, sleep stadification includes sleep spindles in LS. This methodological difference makes not possible to compare these latter results with the results obtained in the present study.

Overall, MID sleeping effects on lactating dams seem to be the consolidation of SWS episodes, increasing the capacity to maintain SWS episodes with few W and LS occurrences. Through the facilitation of GABA-A receptors activity mainly on the reticular activating system ${ }^{60}$, MID might disinhibit certain nuclei of the preoptic area, the key center of NREM sleep. Interesting this same area is crucial in the control of maternal behavior.

\section{GENERAL REMARKS}

The present experiments show that two drugs, HAL and MID, extensively used in clinical studies, affected the maternal behavior and sleep of postpartum rats. Thus, while HAL reduced the number of retrievals, disrupted the grouping of the pups into the nest and increased the time spent nursing, MID reduced pups' grouping without affecting the nursing time. In addition, while HAL increased the time spent in SWS and IS without provoking changes in REM, MID decreased the time spent in LS, increased the time spent in SWS, while IS and REM remained unchanged. These differences may be caused by the pharmacological characteristics of the drugs. In addition, some differences between present and previous studies performed in males might respond to the physiological changes in the female physiology during the postpartum period, stressing the importance of drug studies performed during this unique period in the female' life.

\section{ACKNOWLEDGEMENTS}

Authors have no conflicts of interest to disclose. Funding for this study was partially provided by PEDECIBA.

\section{REFERENCES}

1. Spinelli MG. Postpartum psychosis: detection of risk and management. The American journal of psychiatry. 2009;166(4):405-8.

2. Field T. Postnatal anxiety prevalence, predictors and effects on development: A narrative review. Infant behavior \& development. 2018;51:24-32.

3. Haefely W. The GABA-benzodiazepine interaction fifteen years later. Neurochemical research. 1990;15(2):169-74.

4. Hyttel J, Christensen AV. Biochemical and pharmacological differentiation of neuroleptic effect on dopamine D-1 and D-2 receptors. J Neural Transm Suppl. 1983;18:157-64.

5. ACOG Practice Bulletin: Clinical management guidelines for obstetriciangynecologists number 92, April 2008 (replaces practice bulletin number 87, November 2007). Use of psychiatric medications during pregnancy and lactation. Obstetrics and gynecology 2008:1001-20.

6. Kelly LE, Poon S, Madadi P, Koren G. Neonatal benzodiazepines exposure during breastfeeding. The Journal of pediatrics. 2012;161(3):448-51.

7. Uguz F. A New Safety Scoring System for the Use of Psychotropic Drugs During Lactation. American journal of therapeutics. 2018.

8. Mattmann P, Loepfe M, Scheitlin T, Schmidlin D, Gerne M, Strauch I, et al. Day-time residual effects and motor activity after three benzodiazepine hypnotics. Arzneimittel-Forschung. 1982;32(4):461-5.

9. McClelland GR, Cooper SM, Pilgrim AJ. A comparison of the central nervous system effects of haloperidol, chlorpromazine and sulpiride in normal volunteers. British journal of clinical pharmacology. 1990;30(6):795-803.

10. Fernandez-Guasti A, Ferreira A, Picazo O. Diazepam, but not buspirone, induces similar anxiolytic-like actions in lactating and ovariectomized Wistar rats. Pharmacology, biochemistry, and behavior. 2001;70(1):85-93.

11. Figueira RJ, Peabody MF, Lonstein JS. Oxytocin receptor activity in the ventrocaudal periaqueductal gray modulates anxiety-related behavior in postpartum rats. Behavioral neuroscience. 2008;122(3):618-28.

12. Benedetto L, Rivas M, Pereira M, Ferreira A, Torterolo P. A descriptive analysis of sleep and wakefulness states during maternal behaviors in postpartum rats. Archives italiennes de biologie. 2017;155(3):99-109.

13. Paxinos G, Watson C. The Rat Brain in Stereotaxic Coordinates. 5th ed. San Diego, California: Elsevier Academic Press 2005.

14. Stern JM. Nursing posture is elicited rapidly in maternally naive, haloperidol-treated female and male rats in response to ventral trunk stimulation from active pups. Hormones and behavior. 1991;25(4):504-17.

15. Franca AS, Lobao-Soares B, Muratori L, Nascimento G, Winne J, Pereira $\mathrm{CM}$, et al. D2 dopamine receptor regulation of learning, sleep and plasticity. Eur Neuropsychopharmacol. 2015;25(4):493-504. 
16. Monti JM, Hawkins M, Jantos H, D’Angelo L, Fernandez M. Biphasic effects of dopamine D-2 receptor agonists on sleep and wakefulness in the rat. Psychopharmacology. 1988;95(3):395-400.

17. Ongini E, Bonizzoni E, Ferri N, Milani S, Trampus M. Differential effects of dopamine D-1 and D-2 receptor antagonist antipsychotics on sleepwake patterns in the rat. The Journal of pharmacology and experimental therapeutics. 1993;266(2):726-31.

18. Ongini E, Bo P, Dionisotti S, Trampus M, Savoldi F. Effects of remoxipride, a dopamine D-2 antagonist antipsychotic, on sleep-waking patterns and EEG activity in rats and rabbits. Psychopharmacology. 1992;107(2-3):236-42.

19. Radulovacki M, Sreckovic G, Zak R, Zahrebelski G. Diazepam and midazolam increase light slow-wave sleep (SWS1) and decrease wakefulness in rats. Brain Res. 1984;303(1):194-6.

20. Depoortere H, Francon D, van Luijtelaar EL, Drinkenburg WH, Coenen AM. Differential effects of midazolam and zolpidem on sleep-wake states and epileptic activity in WAG/Rij rats. Pharmacology, biochemistry, and behavior. 1995;51(4):571-6.

21. Lancel M, Cronlein TA, Faulhaber J. Role of GABAA receptors in sleep regulation. Differential effects of muscimol and midazolam on sleep in rats. Neuropsychopharmacology. 1996;15(1):63-74.

22. Stern JM, Taylor LA. Haloperidol inhibits maternal retrieval and licking, but enhances nursing behavior and litter weight gains in lactating rats. Journal of neuroendocrinology. 1991;3(6):591-6.

23. Miller SM, Lonstein JS. Dopamine d1 and d2 receptor antagonism in the preoptic area produces different effects on maternal behavior in lactating rats. Behavioral neuroscience. 2005;119(4):1072-83.

24. Giordano AL, Johnson AE, Rosenblatt JS. Haloperidol-induced disruption of retrieval behavior and reversal with apomorphine in lactating rats. Physiology \& behavior. 1990;48(1):211-4.

25. Stern JM, Johnson SK. Ventral somatosensory determinants of nursing behavior in Norway rats. I. Effects of variations in the quality and quantity of pup stimuli. Physiology \& behavior. 1990;47(5):993-1011.

26. Drewett RF, Trew AM. The milk ejection of the rat, as a stimulus and a response to the litter. Animal behaviour. 1978;26(4):982-7.

27. Lincoln DW, Hill A, Wakerley JB. The milk-ejection reflex of the rat: an intermittent function not abolished by surgical levels of anaesthesia. The Journal of endocrinology. 1973;57(3):459-76.

28. Benedetto L, Rivas M, Cavelli M, Pena F, Monti J, Ferreira A, et al. Microinjection of the dopamine D2-receptor antagonist Raclopride into the medial preoptic area reduces REM sleep in lactating rats. Neuroscience letters. 2017;659:104-9.

29. Benedetto L, Rodriguez-Servetti Z, Lagos P, D'Almeida V, Monti JM, Torterolo P. Microinjection of melanin concentrating hormone into the lateral preoptic area promotes non-REM sleep in the rat. Peptides. 2013;39:11-5

30. Siegel S. Nonparametric Statistics for the Behavioral Sciences. . New York: McGraw-Hill 1956.

31. Pereira M, Ferreira A. Demanding pups improve maternal behavioral impairments in sensitized and haloperidol-treated lactating female rats. Behavioural brain research. 2006;175(1):139-48.

32. De Ryck M, Hruska RE, Silbergeld EK. Estrogen and haloperidolinduced versus handling-related catalepsy in male rats. Pharmacology, biochemistry, and behavior. 1982;17(5):1027-35.

33. Carter CJ, Pycock CJ. Potentiation of haloperidol-induced catalepsy by dopamine agonists: possible involvement of central 5-hydroxytryptamine. Pharmacology, biochemistry, and behavior. 1979;10(4):475-80.

34. Sanberg PR. Haloperidol-induced catalepsy is mediated by postsynaptic dopamine receptors. Nature. 1980;284(5755):472-3.

35. Li M. Antipsychotic drugs on maternal behavior in rats. Behavioural pharmacology. 2015;26(6):616-26.

36. Zhao C, Li M. Sedation and disruption of maternal motivation underlie the disruptive effects of antipsychotic treatment on rat maternal behavior. Pharmacology, biochemistry, and behavior. 2009;92(1):147-56.

37. Silva MR, Bernardi MM, Cruz-Casallas PE, Felicio LF. Pimozide injections into the Nucleus accumbens disrupt maternal behaviour in lactating rats. Pharmacology \& toxicology. 2003;93(1):42-7.

38. Keer SE, Stern JM. Dopamine receptor blockade in the nucleus accumbens inhibits maternal retrieval and licking, but enhances nursing behavior in lactating rats. Physiology \& behavior. 1999;67(5):659-69.
39. Hansen S, Bergvall AH, Nyiredi S. Interaction with pups enhances dopamine release in the ventral striatum of maternal rats: a microdialysis study. Pharmacology, biochemistry, and behavior. 1993;45(3):673-6.

40. Fleming AS, Suh EJ, Korsmit M, Rusak B. Activation of Fos-like immunoreactivity in the medial preoptic area and limbic structures by maternal and social interactions in rats. Behavioral neuroscience. 1994;108(4):724-34.

41. Lonstein JS, Simmons DA, Swann JM, Stern JM. Forebrain expression of $\mathrm{c}$-fos due to active maternal behaviour in lactating rats. Neuroscience. 1998;82(1):267-81.

42. Stern JM, Protomastro M. Effects of low dosages of apomorphine on maternal responsiveness in lactating rats. Pharmacology, biochemistry, and behavior. 2000;66(2):353-9.

43. Dickerman S, Clark J, Dickerman E, Meites J. Effects of haloperidol on serum and pituitary prolactin and on hypothalamic PIF in rats. Neuroendocrinology. 1972;9(6):332-40.

44. Dickerman S, Kledzik G, Gelato M, Chen HJ, Meites J. Effects of haloperidol on serum and pituitary prolactin, LH and FSH, and hypothalamic PIF and LRF. Neuroendocrinology. 1974;15(1):10-20.

45. Mueller GP, Simpkins J, Meites J, Moore KE. Differential effects of dopamine agonists and haloperidol on release of prolactin, thyroid stimulating hormone, growth hormone and luteinizing hormone in rats. Neuroendocrinology. 1976;20(2):121-35.

46. Yasui-Furukori N, Kondo T, Suzuki A, Mihara K, Kaneko S, Otani $K$. Comparison of prolactin concentrations between haloperidol and bromperidol treatments in schizophrenic patients. Progress in neuropsychopharmacology \& biological psychiatry. 2002;26(3):575-8 .

47. Lacasse P, Ollier S. The dopamine antagonist domperidone increases prolactin concentration and enhances milk production in dairy cows. Journal of dairy science. 2015;98(11):7856-64.

48. Monti JM. The effect of haloperidol on the sleep cycle of the cat. Experientia. 1968;24(11):1143.

49. Sivadas N, Radhakrishnan A, Aswathy BS, Kumar VM, Gulia KK Dynamic changes in sleep pattern during post-partum in normal pregnancy in rat model. Behavioural brain research. 2016;320:264-74.

50. Voloschin LM, Tramezzani JH. Milk ejection reflex linked to slow wave sleep in nursing rats. Endocrinology. 1979;105(5):1202-7.

51. Monti JM, Jantos H. The roles of dopamine and serotonin, and of their receptors, in regulating sleep and waking. Progress in brain research. 2008;172:625-46.

52. Zhang SQ, Inoue S, Kimura M. Sleep-promoting activity of prolactinreleasing peptide (PrRP) in the rat. Neuroreport. 2001;12(15):3173-6.

53. Machado RB, Rocha MR, Suchecki D. Brain prolactin is involved in stressinduced REM sleep rebound. Hormones and behavior. 2017;89:38-47.

54. Pereira M, Morrell JI. The changing role of the medial preoptic area in the regulation of maternal behavior across the postpartum period: facilitation followed by inhibition. Behavioural brain research. 2009;205(1):238-48.

55. Rondini TA, Donato J, Jr., Rodrigues Bde C, Bittencourt JC, Elias CF. Chemical identity and connections of medial preoptic area neurons expressing melanin-concentrating hormone during lactation. Journal of chemical neuroanatomy. 2010;39(1):51-62.

56. Meurisse M, Gonzalez A, Delsol G, Caba M, Levy F, Poindron P. Estradiol receptor-alpha expression in hypothalamic and limbic regions of ewes is influenced by physiological state and maternal experience. Hormones and behavior. 2005;48(1):34-43.

57. Frankfurt M, Salas-Ramirez K, Friedman E, Luine V. Cocaine alters dendritic spine density in cortical and subcortical brain regions of the postpartum and virgin female rat. Synapse (New York, NY. 2011;65(9):955-61.

58. Ferreira A, Picazo O, Uriarte N, Pereira M, Fernandez-Guasti A. Inhibitory effect of buspirone and diazepam, but not of 8-OH-DPAT, on maternal behavior and aggression. Pharmacology, biochemistry, and behavior. 2000;66(2):389-96.

59. Yang Y, Qin J, Chen W, Sui N, Chen H, Li M. Behavioral and pharmacological investigation of anxiety and maternal responsiveness of postpartum female rats in a pup elevated plus maze. Behavioural brain research. 2015;292:414-27.

60. Cornett EM, Novitch MB, Brunk AJ, Davidson KS, Menard BL, Urman RD, et al. New benzodiazepines for sedation. Best Pract Res Clin Anaesthesiol. 2018;32(2):149-64. 


\section{Chronobiology in the wild: toolkit to study daily rhythms in free-living animals}

Jefferson T. Silvério ${ }^{1 *}$

Patricia Tachinardi ${ }^{2}$

${ }^{1}$ Departamento de Fisiologia, Instituto de Biociências, Universidade de São Paulo, São Paulo, Brasil.

${ }^{2}$ Departamento de Reprodução Animal, Faculdade de Medicina Veterinária e Zootecnia, Universidade de São Paulo, São Paulo, Brasil.

\begin{abstract}
Since chronobiology's foundation has been laid out in the $60 \mathrm{~s}$, tremendous progress has been made regarding our knowledge about the nature of the circadian clock, its molecular bases and its synchronization by photic and non-photic stimuli. The majority of these studies have been done in laboratory settings, which, although important, lack information about the adaptive value, ecological significance and plasticity of the biological rhythms in the wild. This concern has been raised several times, along with the development of the field. Methodological difficulties have been the biggest challenges of field studies. However, the recent development of new techniques is opening a wide range of opportunities to investigate biological rhythms in free-ranging animals. In this review, we promote an ecological approach to biological clocks and highlight some methods and newer technologies that can be used to study biological clocks in the wild, along with some examples.
\end{abstract}

Keywords: Biological Rhythms; Wild-Clocks; Field And Laboratory; Biologger; Free-Ranging; Activity Patterns 


\section{INTRODUCTION}

Endogenous circadian clocks enable animals to anticipate changes in physiology and behavior to the daily changes in the environment synchronizing their internal state with the 24hour environmental cycles. Our knowledge of the function and mechanism of these clocks has grown extensively in different levels of organizations such as molecular, cellular, tissue and organs. Most of the studies of the mechanisms and nature of the clock itself has been done in the laboratory, under controlled conditions ${ }^{1,2}$. Using the laboratory approach, several endogenous circadian rhythms were described in a diverse range of organisms, from mammals to birds, insects and the molecular clocks of bacteria. However, the physiology and behavior of a laboratory animal can be unrepresentative of what happens in the field ${ }^{3,4}$. Several authors have brought the argument that we know very little about the ecological significance of circadian thythms ${ }^{2,5,6}$. The central argument is that to understand the adaptive significance of circadian rhythms, the diversity of temporal strategies and how flexible circadian rhythms are, we must turn to ecological studies in free-living animals. To fill this gap, several recent studies on animal chronobiology have been carried out in free-living animals. These studies investigate the interplay between the endogenous circadian pacemaker and the biotic and abiotic stimuli acting on the plasticity of overt daily rhythms. These studies have suggested that biological rhythms in the wild are much more flexible than previously thought?

The advancement of chronobiological studies in the field has long been challenged by the difficulty of measuring biological rhythms in nature. In the laboratory, locomotor activity and body temperature $(\mathrm{Tb})$ rhythms have been widely used due to ease of measurement and replicability. Commonly, activity is measured by counting running wheel revolutions, by detecting movement with an infrared motion sensor placed above the animal's cage or by implanting a wireless activity sensor in the animal ${ }^{8}$. Measurements of $\mathrm{Tb}$, in turn, are more invasive and can require more expensive equipment. Usually, Tb sensors are implanted surgically in the peritoneal cavity of the animal. These sensors can either be loggers, in which the data is stored in the device or transmitters, which send data to receivers outside the animal'. When studying free-living animals, however, many of these commonly used methods to study locomotor activity and $\mathrm{Tb}$ rhythms are very difficult or simply impossible to be deployed, being it because of technical or financial drawbacks.

Recent technological advances, such as the miniaturization of sensors and logging devices, have opened a wide variety of opportunities to study biological rhythms in free-living animals. In this mini-review, we will discuss some of the methods being used to assess behavioral and physiological rhythms of freeliving animals in both populational and individual levels. We will focus on methods used in the research of terrestrial mammals, although many of them can also be deployed to other taxa and to aquatic and flying animals ${ }^{10}$. It is important to note that most methods require handling the animal for implanting or externally attaching a device. Thus, researchers should always take into account any possible effects this manipulation can have on the animals and on the data obtained ${ }^{11}$.

\section{CHRONOBIOLOGICAL STUDIES IN FREE-LIVING} ANIMALS

\section{Populational Rhythms}

Most of the early investigations of clocks in the wild have been carried out focusing on populational rhythms, using observational and trapping methods ${ }^{6,12,13}$. Using live-traps to infer activity rhythms can be done by setting a grid of traps in the study site and then counting the number of captures for a chosen time interval. For example, using hourly record of vole (Microtus arvalis) trappings in the wild it was possible to record the pattern of daily activity in these species and their ultradian components ${ }^{14}$. If a higher temporal resolution is required, it is possible to combine live-trapping and direct observations to record the exact time the animals were trapped. Using this approach it was shown that Kangaroo rats (genus Dipodomys) display seasonal changes in their start and end time of activity, which might be related to the length changes of sunset ${ }^{12}$. Both observational and live-trapping methods are limited in the range of habitats and diversity of animals they can be applied to. Observational studies have to be done in diurnal species living in open habitats, otherwise, it becomes unfeasible to observe the study species. Small nocturnal animals and animals living in habitats with dense vegetation are not well suited for this technique. On the other hand, trapping studies face the problems of how easily individuals of the study species can be trapped ${ }^{6}$. Both of the abovementioned examples were done in species that are relatively easy to capture in live traps. Kangaroo rats, for example, do not exhibit an aversion to the traps and manipulation. Voles, in turn, leave recognizable tracks aboveground that can guide trap placement and increase trapping success.

More recently, the use of time-stamped camera-trap images has expanded the study of population activity rhythms to species that cannot be easily observed or captured ${ }^{15,16}$. Moreover, this non-invasive technique can be used to simultaneously monitor several species that occur in the same area. For example, Mendes and colleagues ${ }^{17}$ did an extensive camera-trap study to evaluate the changes in daily activity rhythms of 17 rainforestdwelling mammal species in response to human disturbance. They found out that there was a shift in the timing of activity in highly disturbed areas, with some species becoming more nocturnal and others more diurnal. Species that were susceptible to preying or hunting were more likely to show activity time shifts. Possible drawbacks of using camera-traps are camera placement bias, detectability of the animals in the camera and the assumption that all individuals of the population will display peak activity at the same time ${ }^{15,16}$

\section{Individual behavioral rhythms}

In general, to study individual daily activity patterns it is necessary to continuously follow and record movement and behavior of the same individual for many days ${ }^{18}$, which can be very challenging in the field. Individual monitoring can be done using direct observations ${ }^{19,20}$, but this method restricts the monitoring of the animal to the times when it is visible. 
For continuous measurements, it is usually required to attach a device to the animal. Among the earliest measurements of individual activity rhythms using animal-borne devices were those using radio telemetry ${ }^{21,22}$. When using this technique, the animal is equipped with a radio telemetry collar that continuously transmits a pulse in a certain radiofrequency that can be picked up by a radio receiver tuned to the same frequency. This way it is possible to locate the animal in the field during defined time intervals. Daily activity patterns are inferred by the size of spatial displacements between these time points throughout day and night. Due to the availability of small and lightweight transmitters, this technique can be used on mammals of nearly any size ${ }^{23}$. Telemetry studies are often done manually, thus being very labor-intense and timeconsuming. There are automated telemetry systems, which are a feasible alternative to measure activity patterns, as long as the technical and financial requirements to set up the system can be met and errors due to small body size and large home ranges are taken into account ${ }^{24}$. Another method that can be used to assess activity patterns based on animal movement are satellite trackers, which previously could only be used in large-sized animals, but are currently being miniaturized ${ }^{25}$. An important advantage of this technique is that data can be obtained remotely, with no need to locate the animal after the tag is deployed.

Individual-level rhythms can also be measured with newer biologging devices, which are attached to the animal's body and can record onboard physiological and behavioral parameters including activity, $\mathrm{Tb}$, heart rate and skin humidity ${ }^{26,27}$. Given the advancements in the biologging technologies, devices are now smaller and capable of recording multiple parameters in a frequency of hundreds of points per second, providing insight into the details of the animal's life ${ }^{26}$. Due to the onboard memory, the main drawback of biologgers is the need to recapture the animals to obtain the data.

Examples of biologging devices used to monitor animal behavior in chronobiological studies include lightloggers and accelerometers. Lightloggers are devices that were originally designed to be used for geolocation in bird migration studies but are of particularly interesting for chronobiology. Lightloggers are being used to conduct research on a number of small rodents, recording their temporal patterns of exposure to light. Studies that are benefitted by the use of lightloggers include research on diurnal animals that retreat to nests, subterranean or semifossorial animals and hibernating animals in which exposure to light means the animals are active above ground. Accelerometers, in turn, are tri-axial devices that continuously record fine-scale movement. The data generated by accelerometers can be used to quantify gross activity timing, identify behaviors based on movement patterns, quantify time-activity budgets and indirectly derive energy expenditure ${ }^{26-28}$.

Lightloggers were used to investigate the daily and seasonal patterns of activity in wild red squirrels (Tamiasciurus budsonicus) and arctic ground squirrels (Urocitellus parryiz) ${ }^{29}$. Using these devices, they could calculate the total time outside of the nest, the number of activity bouts per day, time of the first emergence in the morning and return to nest at night.
They showed that the level of activity was flexible and correlated with changes in the thermoregulatory conditions of the environment. On a different rodent species, the tuco-tuco (genus Ctenomys), a combination of lightloggers and accelerometry was used to investigate the activity patterns of a subterranean rodent in outdoor enclosures ${ }^{30}$. In this study, accelerometers were used to record gross motor activity while lightloggers recorded time on surface. A combination of both methods, therefore, could discriminate levels of aboveground activity and belowground activity when the animal is inside its burrow system. The study showed that time of day and temperature were the main environmental conditions modulating time spent on the surface ${ }^{30}$ and that there was a shift from diurnal to nocturnal levels of activity when the same animals were transferred from outdoor enclosure to controlled laboratory conditions ${ }^{30,31}$. Although this study was done in outdoor enclosures, this approach can also be used in freeranging animals. Shifts in the timing of activity are also known to happen in other species of rodents besides Ctenomys sp.,32 and are among the most dramatic discrepancies between rhythms in the field and lab. Biologgers are a helpful addition to approach this phenomenon at the individual level and boost this investigation. The examples provided here highlight possible scenarios where the use of biologging devices can be used to reveal the intimacy and complexity of rhythms in the wild, investigating chronobiological questions and the flexibility of circadian and seasonal rhythms.

Other alternative technologies can be used to assess rhythms in the wild, such as RFID (Radio Frequency Identification) and newly developed ones such as the BATS system. RFID are small tags that can be used to identify individuals in a population Passive RFID tags do not require their battery to operate and are powered by the energy from the reader's radio waves. They are largely used to identify farm and laboratory animals, being very small and attached either externally or subcutaneously. RFID tags can be used to record activity rhythms by placing readers in locations that the animals visit regularly, such as nests or feeders. This setup allows the recording of the rhythmicity of visits to a specific location where the reader is placed ${ }^{33}$. Although this technique is usually deployed in outdoor enclosures it could be used in free-living animals that have fixed nests or feeding locations. The BATS system is an interesting technology that is fully automated ${ }^{34,35}$. It is composed of small lightweight proximity sensors and a set of receiver stations that are distributed in the study site. The proximity sensors can record interactions between two animals in a sampling rate of seconds and then transmit these data to the base stations. This system can also be used to derive movement trajectory even in structurally complex habitats ${ }^{35}$. Ripperger and colleagues have used this system to study the behavioral ecology of bats, such as the mother-offspring interactions in noctule bats (Nyctalus noctule) $)^{36}$ and the social structure of the common vampire bats (Desmodus rotundus) ${ }^{37}$. Although the BATS system was developed to investigate a different set of questions it seems to be a great new technology to assist in the study of reproduction, social synchronization and the social influences on biological rhythms. 


\section{Physiological rhythms}

Biologgers can also be used to assess daily rhythms of physiological variables, enabling the investigation of physiological adjustments to the changes in environmental conditions faced by free-living animals. Tb is the physiological variable most studied in wild chronobiology. It is usually measured and recorded by temperature loggers implanted into the abdomen. This method was used to monitor $\mathrm{Tb}$ in freeliving arctic ground squirrels (Urocitellus parryii) throughout the year, showing that in these animals $\mathrm{Tb}$ is arrhythmic during hibernation but displays an entrained 24-hour rhythm in the active season even during the weeks of constant $\operatorname{sun}^{38}$. Monitoring of $\mathrm{Tb}$ was also used to record dramatic shifts from diurnality to nocturnality when golden spiny mice (Acomys russatus) were transferred from large outdoor enclosures to the laboratory ${ }^{39}$. This study is a clear example of how the assessment of physiological rhythms can be important in the investigation of the plasticity of biological rhythms, since the same physiological variable can be measured in both conditions, as opposed to measuring different types of behavioral rhythms under field and laboratory conditions (e.g. foraging in the field and wheel running in the laboratory).

In the laboratory, energy expenditure rhythms can be assessed by measuring hourly rates of oxygen consumption using respirometry chambers, for instance, which is not possible in free-living animals. Since heart rate can be a proxy to energy expenditure, it is a valuable physiological variable in chronobiological studies involving energetics. Heart rate sensors can be attached to the skin, implanted subcutaneously ${ }^{40}$ or even placed non-surgically in the reticulum of ruminants ${ }^{41}$. These devices can either be loggers ${ }^{40}$ or transmitters ${ }^{41}$.

Brain electrical activity is also of interest to chronobiological studies since it can be used to investigate sleep patterns. Recently, miniature loggers have been developed to measure electrophysiological brain activity ${ }^{42,43}$. Studies using these loggers have, for instance, provided insights into the ecology of sleep in sloths ${ }^{44}$ and even indicated that birds can sleep during flight ${ }^{45}$.

\section{CONCLUSION}

In this mini-review, we highlighted some, but not all, methods that can be applied in chronobiological studies in the wild. There is a range of new devices and techniques currently being developed that could be applied to freeliving animals to assess biological rhythms. The combination of different devices can also be used to discriminate and investigate how some behaviors or physiology interact, even at the cellular and molecular levels ${ }^{46}$. The fast methodological advancement and the increasing interest of chronobiologists in field studies are key aspects to narrow the gap between what we know about the circadian clocks in the lab and what we know about their functional significance in nature.

\section{ACKNOWLEDGEMENTS}

We would like to thank Gisele Oda for the revision and suggestions that improved the manuscript; Danilo Flôres, Milene Jannetti and Giovane Improta for discussion of everything chronobiology; and the organizing committee of the XV Latin American Symposium on Chronobiology. JTS was funded in part by the Coordenação de Aperfeiçoamento de Pessoal de Nivel Superior - Brasil (CAPES) - Finance Code 001, CAPES scholarship (88882.377383/2019-01) and additional grant from Fundação de Amparo à Pesquisa do Estado de São Paulo (FAPESP) (2017/19680-2). PT was funded by FAPESP fellowship 2017/22973-1.

\section{REFERENCES}

1. Morgan E (2004) Ecological Significance of Biological Clocks. Biological Rhythm Research 35:3-12

2. Enright JT (1970) Ecological Aspects of Endogenous Rhythmicity. Annu Rev Ecol Syst 1:221-238

3. Willmer P, Stone G, Johnston IA (2005) Environmental physiology of animals, 2nd ed. Blackwell Pub, Malden, Mass

4. Calisi RM, Bentley GE (2009) Lab and field experiments: Are they the same animal? Hormones and Behavior 56:1-10

5. Helm B, Visser ME, Schwartz W, Kronfeld-Schor N, Gerkema M, Piersma T, Bloch G (2017) Two sides of a coin: ecological and chronobiological perspectives of timing in the wild. Phil Trans R Soc B 372:20160246

6. Halle S, Stenseth NC (eds) (2000) Activity patterns in small mammals: an ecological approach. Springer, Berlin; New York

7. Hut RA, Kronfeld-Schor N, van der Vinne V, De la Iglesia H (2012) In search of a temporal niche: Enviromental Factors. In: Progress in Brain Research. Elsevier, pp 281-304

8. Blanchong JA, McElhinny TL, Mahoney MM, Smale L (1999) Nocturna and diurnal rhythms in the unstriped Nile rat, Arvicanthis niloticus. J Biol Rhythms 14:364-377

9. Weinert D, Waterhouse J (1998) Diurnally changing effects of locomotor activity on body temperature in laboratory mice. Physiol Behav 63:837-843

10. Dominoni DM, Åkesson S, Klaassen R, Spoelstra K, Bulla M (2017) Methods in field chronobiology. Phil Trans R Soc B 372:20160247

11. Chmura HE, Glass TW, Williams CT (2018) Biologging Physiological and Ecological Responses to Climatic Variation: New Tools for the Climate Change Era. Front Ecol Evol 6:92

12. Kenagy GJ (1976) The periodicity of daily activity and its seasonal changes in free-ranging and captive kangaroo rats. Oecologia 24:105-140

13. Daan S, Slopsema S (1978) Short-term rhythms in foraging behaviour of the common vole,Microtus arvalis. J Comp Physiol 127:215-227

14. Gerkema M, Daan S (1985) Ultradian rhythms in behavior The case of the common vole (Microtus arvalis). https://doi.org/10.1007/978-3 642-70483-3_3

15. Rowcliffe JM, Kays R, Kranstauber B, Carbone C, Jansen PA (2014) Quantifying levels of animal activity using camera trap data. Methods in Ecology and Evolution 5:1170-1179

16. Frey S, Fisher JT, Burton AC, Volpe JP (2017) Investigating animal activity patterns and temporal niche partitioning using camera-trap data: challenges and opportunities. Remote Sensing in Ecology and Conservation 3:123-132

17. Mendes CP, Carreira D, Pedrosa F, Beca G, Lautenschlager L, Akkawi P, Bercê W, Ferraz KMPMB, Galetti M (2020) Landscape of human fear in Neotropical rainforest mammals. Biological Conservation 241:108257

18. Daan S (1981) Adaptive Daily Strategies in Behavior. In: Handbook of Behavioral Neurobiology. Plenum Press, p 563

19. Everts LG, Strijkstra AM, Hut RA, Hoffmann IE, Millesi E (2004) Seasonal variation in daily activity patterns of free-ranging European ground squirrels (Spermophilus citellus). Chronobiol Int 21:57-71

20. Tomotani BM, Flores DEFL, Tachinardi P, Paliza JD, Oda GA, Valentinuzzi VS (2012) Field and Laboratory Studies Provide Insights into the Meaning of Day-Time Activity in a Subterranean Rodent (Ctenomys aff. knighti), the Tuco-Tuco. PLoS ONE 7:e37918

21. Ables ED (1969) Activity Studies of Red Foxes in Southern Wisconsin. The Journal of Wildlife Management 33:145 
22. Hut RA, van Oort BEH, Daan S (1999) Natural Entrainment without Dawn and Dusk: The Case of the European Ground Squirrel ( Spermophilus citellus ). J Biol Rhythms 14:290-299

23. Naef-Daenzer B (2005) Miniaturization $(0.2 \mathrm{~g})$ and evaluation of attachment techniques of telemetry transmitters. Journal of Experimental Biology 208:4063-4068

24. Kays R, Tilak S, Crofoot M, et al (2011) Tracking Animal Location and Activity with an Automated Radio Telemetry System in a Tropical Rainforest. Comput J 54:1931-1948

25. Wikelski M, Cooke SJ (2006) Conservation physiology. Trends in Ecology \& Evolution 21:38-46

26. Ropert-Coudert Y, Wilson RP (2005) Trends and perspectives in animalattached remote sensing. Frontiers in Ecology and the Environment 3:437-444

27. Rutz C, Hays GC (2009) New frontiers in biologging science. Biol Lett 5:289-292

28. Brown DD, Kays R, Wikelski M, Wilson R, Klimley AP (2013) Observing the unwatchable through acceleration logging of animal behavior. Animal Biotelemetry 1:20

29. Williams CT, Wilsterman K, Kelley AD, Breton AR, Stark H, Humphries MM, McAdam AG, Barnes BM, Boutin S, Buck CL (2014) Light loggers reveal weather-driven changes in the daily activity patterns of arboreal and semifossorial rodents. Journal of Mammalogy 95:1230-1239

30. Jannetti MG, Buck CL, Valentinuzzi VS, Oda GA (2019) Day and night in the subterranean: measuring daily activity patterns of subterranean rodents (Ctenomys aff. knighti) using bio-logging. Conserv Physiol. https://doi.org/10.1093/conphys/coz044

31. Tachinardi P, Tøien $\varnothing$, Valentinuzzi VS, Buck CL, Oda GA (2015) Nocturnal to Diurnal Switches with Spontaneous Suppression of Wheel-Running Behavior in a Subterranean Rodent. PLoS ONE 10:e0140500

32. Kronfeld-Schor N, Visser ME, Salis L, van Gils JA (2017) Chronobiology of interspecific interactions in a changing world. Phil Trans R Soc B 372:20160248

33. Vinne V van der, Tachinardi P, Riede SJ, Akkerman J, Scheepe J, Daan S, Hut RA (2019) Maximising survival by shifting the daily timing of activity. Ecology Letters 22:2097-2102

34. Duda N, Nowak T, Hartmann M, et al (2018) BATS: Adaptive Ultra Low Power Sensor Network for Animal Tracking. Sensors 18:3343

35. Ripperger SP, Carter GG, Page RA, et al (2019) Thinking small: nextgeneration sensor networks close the size gap in vertebrate biologging. bioRxiv 767749
36. Ripperger S, Günther L, Wieser H, Duda N, Hierold M, Cassens B, Kapitza R, Koelpin A, Mayer F (2019) Proximity sensors on common noctule bats reveal evidence that mothers guide juveniles to roosts but not food. Biology Letters 15:20180884

37. Ripperger SP, Carter GG, Duda N, Koelpin A, Cassens B, Kapitza R, Josic D, Berrío-Martínez J, Page RA, Mayer F (2019) Vampire Bats that Cooperate in the Lab Maintain Their Social Networks in the Wild. Current Biology 29:4139-4144.e4

38. Williams CT, Sheriff MJ, Schmutz JA, Kohl F, Tøien Ø, Buck CL, Barnes BM (2011) Data logging of body temperatures provides precise information on phenology of reproductive events in a free-living arctic hibernator. J Comp Physiol B 181:1101-1109

39. Levy O, Dayan T, Kronfeld-Schor N (2007) The relationship between the golden spiny mouse circadian system and its diurnal activity: an experimental field enclosures and laboratory study. Chronobiol Int 24:599-613

40. Tøien $\varnothing$, Blake J, Edgar DM, Grahn DA, Heller HC, Barnes BM (2011) Hibernation in Black Bears: Independence of Metabolic Suppression from Body Temperature. Science 331:906-909

41. Signer C, Ruf T, Schober F, Fluch G, Paumann T, Arnold W (2010) A versatile telemetry system for continuous measurement of heart rate, body temperature and locomotor activity in free-ranging ruminants. Methods Ecol Evol 1:75-85

42. Massot B, Arthaud S, Barrillot B, Roux J, Ungurean G, Luppi P-H, Rattenborg NC, Libourel P-A (2019) ONEIROS, a new miniature standalone device for recording sleep electrophysiology, physiology, temperatures and behavior in the lab and field. Journal of Neuroscience Methods 316:103-116

43. Vyssotski AL, Serkov AN, Itskov PM, Dell'Omo G, Latanov AV, Wolfer DP, Lipp H-P (2006) Miniature neurologgers for flying pigeons: multichannel EEG and action and field potentials in combination with GPS recording. J Neurophysiol 95:1263-1273

44. Rattenborg NC, Voirin B, Vyssotski AL, Kays RW, Spoelstra K, Kuemmeth F, Heidrich W, Wikelski M (2008) Sleeping outside the box: electroencephalographic measures of sleep in sloths inhabiting a rainforest. Biol Lett 4:402-405

45. Rattenborg NC, Voirin B, Cruz SM, Tisdale R, Dell'Omo G, Lipp H-P, Wikelski M, Vyssotski AL (2016) Evidence that birds sleep in mid-flight. Nat Commun 7:1-9

46. Jansen HT, Leise T, Stenhouse G, Pigeon K, Kasworm W, Teisberg J Radandt T, Dallmann R, Brown S, Robbins CT (2016) The bear circadian clock doesn't 'sleep' during winter dormancy. Frontiers in Zoology 13:42 


\section{What, how and (especially) when: chronoparmacological approach in cancer therapies}

Laura Lucía Trebucq ${ }^{1}$

Luciano Marpegan ${ }^{2}$

Diego Andres Golombek ${ }^{1}$

Juan José Chiesa ${ }^{1 *}$

${ }^{1}$ Laboratorio de Cronobiología, Departamento de Ciencia y Tecnología, Universidad Nacional de Quilmes, Bernal, Buenos Aires, Argentina. ${ }^{2}$ Instituto de Física Médica, Comisión Nacional de Energía Atómica, Bariloche, Río Negro, Argentina.

\begin{abstract}
Most physiological and behavioural processes in mammals show 24-h period oscillations regulated by an endogenous circadian clock. Drugs pharmacokinetics and pharmacodynamics are not the exception. Determining the time of day when the therapeutic effect of a certain drug is maximum and, in turn, its side effects are minimum, is the main objective of chronopharmacology. The daily oscillations in processes necessary for the absorption, metabolization, distribution and excretion of drugs determine the chronopharmacokinetics, while the oscillations of the targets, receptors, enzymes and signaling systems of these drugs determine the chronopharmacodynamics. Two concepts are important when analyzing the therapeutic value of a drug: toxicity and effectiveness. Indeed, studying the dependence of drug effect on the circadian time of administration may help to determine the putative daily variation in their therapeutic index. Many anti-tumor compounds have circadian variations in their pharmacokinetics and dynamics, depending on the time of administration. Nevertheless, not many of them are currently administered according to a chronopharmacological approach. Studying the timedependent effect of oncological drugs is essential to generate therapeutic advances in cancer treatment.
\end{abstract}

Keywords: Chronotherapy; Tumor; Circadian Rhythms; Pharmacokinetics; Pharmacodynamics
Corresponding author:

Juan José Chiesa

E-mail: juanjosechiesa@gmail.com 


\section{INTRODUCTION}

Almost all organisms have a circadian (i.e., approximate 24-h period) clock, which allows them to synchronize to cyclic environmental variables that change along the day. Since the rotation of the Earth keeps a stable 24-h period, the light-dark (LD) cycle becomes an efficient circadian synchronizer (i.e., zeitgeber or "time giver") for most epigean species ${ }^{1}$. In mammals, the main circadian oscillator is located in the suprachiasmatic nuclei of the hypothalamus (SCN); these nuclei coordinate and synchronize circadian oscillators in peripheral tissues, directly through endocrine and autonomic pathways, or indirectly through behavioral rhythms ${ }^{2}$. In addition to the photic transitions of the LD cycle, other daily environmental stimuli can act as zeitgebers in some species, such as cycles in temperature, food availability, social interactions, etc. ${ }^{1}$.

The main function of the central SCN clock is to synchronize the organism to the environmental cycles, generating overt rhythms in behavioral, physiological, and biochemical variables. This implies the need of secondary clocks downstream of the SCN. Indeed, the vast majority of cells and organs in the body are capable of generating regular cycles of circadian gene expression, thus becoming "peripheral oscillators" ${ }^{\text {. These }}$ oscillators are hierarchically coupled in the circadian system and regulate specific circadian functions at peripheral tissues or organs ${ }^{4}$. They are coordinated by neuroendocrine, endocrine (e.g., melatonin, glucocorticoids) and autonomic outputs of the SCN, as well as behavioral outputs generating peripheral signals $^{5,6}$. For example, since the feeding-fasting rhythm is a potent zeitgeber for peripheral oscillators involved in energy metabolism, the liver circadian clock can be forced to oscillate in phase with food availability in restricted feeding protocols, independently of the SCN $\operatorname{clock}^{7,8}$.

The study of the variability along the day in the action of drugs (i.e., chronopharmacology), is an established clinical and experimental field in chronobiology. The daily oscillations in physiological and molecular processes generate rhythms in the chronopharmacokinetic processes (i.e., chronopharmacokinetics), while the oscillations in the targets of these drugs (receptors, enzymes and effector signaling systems) determine the chronopharmacodynamics of drug treatment ${ }^{9-11}$. Thus, detecting these oscillations could help to manage the toxicity and effectiveness of the different drugs by controlling their time of administration. This is the main goal of chronopharmacology: to determine the time of day when the therapeutic effect of a certain drug is maximum and, in turn, to minimize its side effects.

In addition, several evidences show the importance of circadian clocks in tumor progression. It has already been shown that the pharmacokinetics and dynamics of various antitumor drugs show circadian variations, depending on the time of day they are administered ${ }^{12,13}$. One of the goals of a designed chronochemotherapy is to take advantage of the differential responses of normal and tumor cells in order to reduce side effects.
Such cells might differ in their circadian rhythmicity ${ }^{14,15}$, generating a differential sensitivity to drugs related to contrasting pharmacokinetic and dynamic mechanisms. Within these differential mechanisms, the production of toxic metabolites at different times of the day, and circadian variations in cell defense mechanisms, can be important for chronopharmacology ${ }^{16,17}$. Therefore, in order to achieve minimal toxicity on normal cells (and maximal on tumors), the time of administration might be crucial for optimal therapies.

In this paper we review the actual knowledge regarding chronopharmacology of anti-tumor therapies at both cellular and system levels, considering its implications for future cancer chronotherapies.

\section{MOLECULAR COMPONENTS OF THE MAMMALIAN CIRCADIAN CLOCK.}

The circadian system is a hierarchical, multi oscillator network that coordinates daily rhythms in physiology and behavior. At the cellular level, the core circadian mechanism involves positive and negative transcriptional-translational feedback loops generated by a group of highly conserved clock genes $^{18}$. The positive loop is based on a transcription factor formed by proteins encoded by clock (Circadian Locomotor Output Cycles Kaput) and aryl hydrocarbon receptor nuclear translocator (ARNT)-like 1 (or brain and muscle ARNT-like 1, bmal1) genes. The CLOCK and BMAL1 proteins form a heterodimer that activates the transcription of the period (per) 1 , 2 and 3 , and cryptochrome (cry) 1 and 2 genes, in addition to other clock-controlled genes. Such activation can occur by binding the heterodimer to specific E-Box sites in the per and $c r y$ genes. The negative feedback loop is carried out by the heterodimer formed by the PER1-2 and CRY1-2 proteins, which is translocated to the nucleus and binds to the CLOCK-BMAL1 heterodimer to inhibit its own transcription. A whole cycle of feedback lasts approximately $24 \mathrm{~h}$, constituting the circadian molecular clock ${ }^{19}$. In addition to the primary feedback loop, there is a secondary negative feedback loop that involves nuclear receptors related to retinoic acid, REV-ERB- $\alpha$ and ROR- $\alpha$. These genes are activated by the CLOCK-BMAL1 heterodimer (Fig.1). In turn, the proteins encoded by REV-ERB- $\alpha$ and ROR- $\alpha$ compete to bind to the RORE response elements, present in the bmal1 promoter, inhibiting or activating their expression, respectively. These proteins, therefore, regulate the expression of bmaln. While this secondary loop is not essential for the molecular clock, it is hypothesized that it provides robustness to the system ${ }^{20,21}$. While mRNA levels of per1, 2 and 3, cry 1 and 2, Roro, and RevErbo have their maximum during the day, Bmal1 mRNA levels exhibit their maximum during the night $\mathrm{t}^{20}$. Post-transcriptional modifications and degradation of clock proteins are essential to determine the periodicity of the clock. For instance, the casein

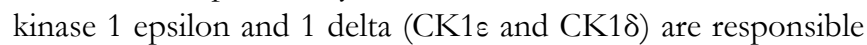
for the phosphorylation of PERs and CRYs to promote their degradation by ubiquitination ${ }^{22}$. 


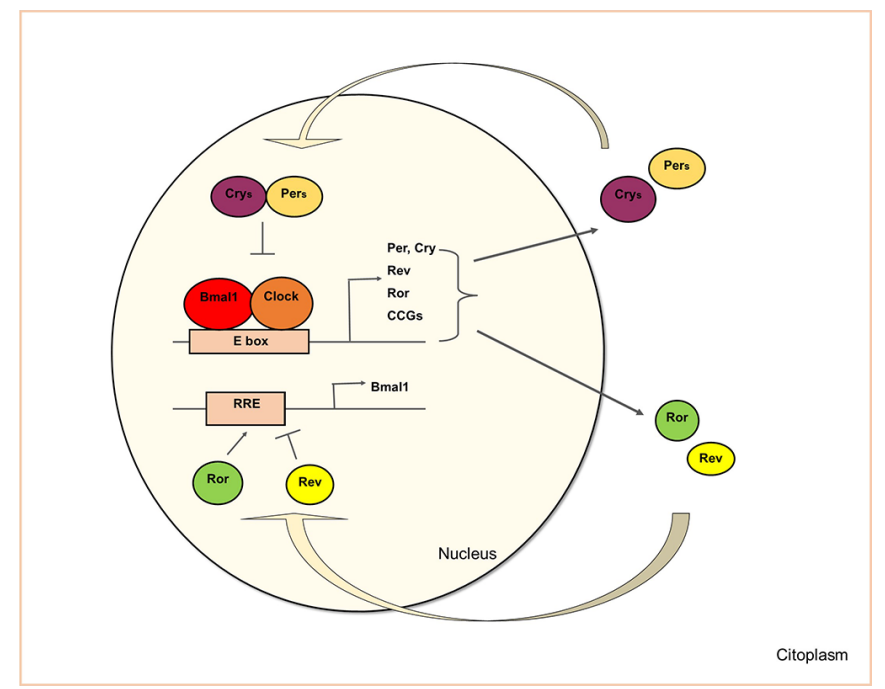

Figure 1. Model of the molecular circadian clock of mammals. The main feedback loop is carried out by CLOCK and BMAL1. These proteins heterodimerize and initiate the transcription of the genes period (per) 1,2 and 3, and cryptochrome (cry) 1 and 2, in addition to other genes controlled by the clock. The negative feedback loop is represented by the heterodimer formed by PER 1 or 2 and CRY 1 or 2, which translocates to the nucleus and inhibits its own transcription, binding the CLOCK-BMAL1 heterodimer. The proteins encoded by REV-ERB- $\alpha$ (REV) and ROR- $\alpha$ (ROR) form the secondary negative feedback loop, and compete to bind to the RRE response elements, present in the bmal1 promoter, inhibiting or activating its expression, respectively.

\section{THE LINK BETWEEN CIRCADIAN RHYTHMS AND CANCER.}

There is evidence about the importance of the circadian clock in tumor progression. Chronic disruption of biological rhythms due to deficient circadian regulation and/or photic synchronization generates physiological alterations that could lead to pathology. For instance, it has been observed in mice that imposing frequent advances of the LD cycle (i.e, chronic jet-lag) generates behavioral desynchronization promoting circadian misalignment of peripheral organs, increasing angiogenesis and accelerating tumor progression ${ }^{23-25}$. Significantly, it has been reported that work in rotating shifts augments the incidence of certain types of cancers ${ }^{26,27}$, being declared a risk factor by the World Health Organization ${ }^{24}$.

Tumor growth can be regulated by genes that are part of the molecular circadian clock and are also related to pathways important in the progression of this disease, such as the cell division cycle and the DNA repair system, among others ${ }^{16,17}$. For instance, in human osteosarcoma cells, G1/S cell cycle regulators mediate effects of circadian dysregulation on tumor growth and provide targets for timed anticancer treatment ${ }^{28,29}$, among other examples. On the other hand, stable circadian rhythms were reported in various tumor cell lines, as well as in primary tumor cells cultured from biopsies ${ }^{14,15,30}$. However, variability in the circadian period was observed depending either on the type of cell or on the patient: $24-\mathrm{h}$ rhythms were found in glioblastoma patient-derived cells ${ }^{15}$, in low-grade breast cancer cells ${ }^{31}$ and colorectal cancer cells ${ }^{32}$.
It is possible that tumor development at the molecular level is linked to disrupted circadian control of the cell cycle, which involves many factors, such as phosphorylation of kinases, their binding to cyclins, anaphase-promoting complexes that promote cyclin degradation and activation of various key genes and its pathways, such as wee1, $c d c 2, p 21$ and $p 53(16,17,33,34)$. It has already been shown that BMAL1 induces the expression of WEE1 (16) and other cell'cycle genes, directly or indirectly, as well as those coding for Cyclin $\mathrm{D}^{35}$, Cyclin B, ${ }^{36}$ and p21 ${ }^{37}$ (Fig.2).

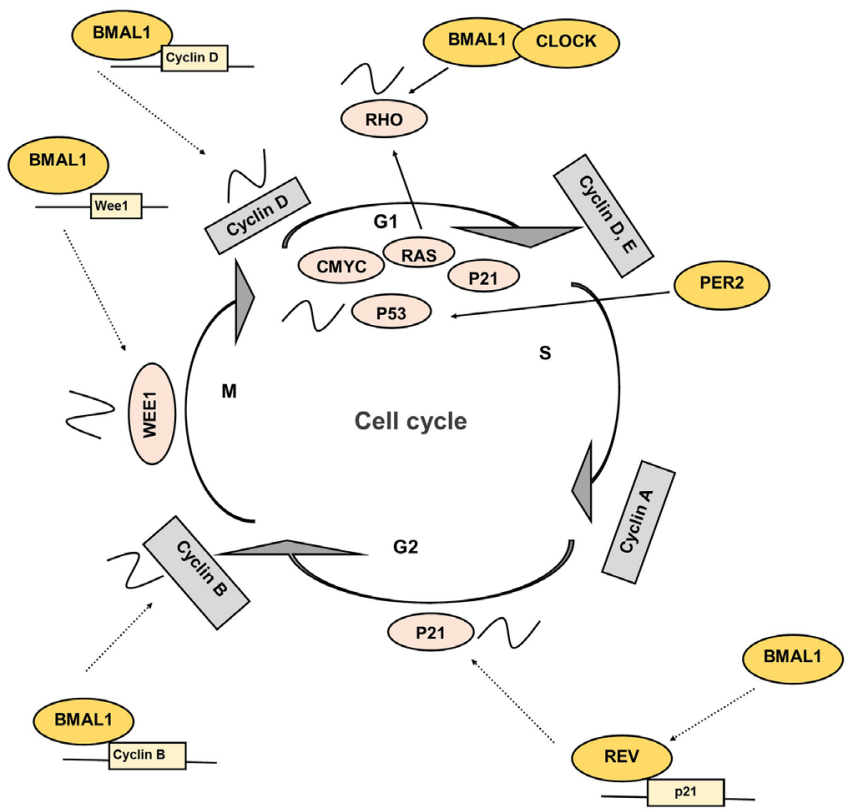

Figure 2. Circadian control of the cell cycle. BMAL1 directly controls the expression of the inhibitor kinase Wee1, Cyclin B (triggers G2/M transition), Cyclin E (triggers G1/S transition) and Cyclin D (present in M and G1) (dotted arrows). It also controls indirectly the inhibitor kinase p21, via REV. BMAL1 controls the expression of the clock gene REV, which controls the expression of $\mathrm{p} 21$. CLOCK and BMAL1 stabilize the monomeric GTPase RHOA (particularly in uterine cancer cells), a protein that is downstream RAS pathway, by controlling the components of the RhoA-ROCK-CFL pathway in order to promote F-actin formation and cell proliferation. PER2 modulates P53 stability (full arrows). Proteins that display circadian rhythms are shown. Only some of the main regulatory interactions are shown in this simplified scheme.

A large number of clinical studies showed aberrant expression of clock genes in various types of cancer. Per1 and per2 have been shown to be downregulated in breast cancer, human gastric cancer, head, neck and oral squamous carcinoma, and prostate cancer ${ }^{38-41}$. Several studies in glioblastoma cell lines showed a low expression of cry 1 and 2 and an overexpression of clock $^{42}$. Kiessling et al. found that B16 murine skin carcinoma cells exhibits suppressed oscillation of clock genes, but treatment with dexametasone or forskolin restored these rhythms, which resulted in fewer cells in the S phase and more in the G1 phase ${ }^{43}$. Therefore, in several tumoral cells a deregulation or aberrant expression in the clock genes implies a deregulation of the cell cycle, which could ultimately involve tumor development ${ }^{44}$. There is evidence that indicates that the circadian genes per 1 and per 2 inhibit the proliferation of tumor cells by controlling the cell cycle, thus acting as tumor suppressors ${ }^{17,34}$. 
In vivo, it was shown that PER2 deficiency increased tumor incidence generated by irradiation ${ }^{45}$ and accelerated lung cancer progression in nude mice ${ }^{46}$. The effect of the per1 and per2 genes in the inhibition of tumor cell proliferation may be due to alterations in the expression of cyclin D1 (an important protein in the progression of the G1-S phase), as well as alterations in the expression of wee1 and $c d k 1$ genes (key regulators at the G2-M control point $)^{47}$. Fu et al. reported that per1 downregulation results in a decreased expression of $p 53, p 16$ and $p 21$, and an increased expression of Cyclin D1, B1 and $\mathrm{E}^{32}$. In addition, it has also been shown that PER2 modulates p53 stability (Fig.2) and induces the transcription of p53 target genes, such as BAX and $\mathrm{SFN}^{48}$, demonstrating that Per2 plays an important role in regulating p53 pathway and tumor suppression.

Similar results were found for BMAL1: this protein acts as a tumor suppressor gene by positively regulating the p53 tumor suppressor pathway in pancreatic cancer ${ }^{49}$. When studying bmal1 knockouts, different carcinogenic effects were found. Korkmaz and colleagues described an increase in apoptosis in different cell lines of breast cancer with deficient bmal1 gene expression ${ }^{50}$. Nevertheless, Kettner et al. reported that hepatocyte-specific loss of bmal1 enhances the formation of spontaneous hepatocellular carcinoma ${ }^{51}$. These results are coincident with the idea that bmal1 may act in some cancers as a tumor suppressor gene while, in other ones, the same gene may sustain tumor growth and proliferation.

On the other hand, clock genes can promote cell proliferation in different types of cancer, by regulating different proliferative pathways. Yaping et al. showed that clock is upregulated in SW480 colorectal cancer cells and that, when overexpressed, it enhances migration, suggesting that this protein regulates migration in this tumor cell type. Clock overexpression also upregulated the expression of tumor angiogenesis related genes, such as HIF-1a and VEGF, thus contributing to tumor malignancy ${ }^{52}$. Ma et al. demonstrated that CLOCK and BMAL1 stabilize the monomeric GTPase RhoA in uterine cancer cells, by controlling the components of the RhoA-ROCK-CFL pathway in order to promote F-actin formation and cell proliferation, invasion and migration ${ }^{53}$ (Fig.2). In addition, El-Athman et al. reported that colon cancer cells with Ras overexpression showed a 2-h longer period than wild type cells ${ }^{54}$.

Indeed, several results suggest that the role of clock genes in tumor progression is complex and depends not only on the type of tumor, but also on the type of clock gene and the molecular oscillation that the cell line displays: some clock genes can be promoters of tumor progression in certain types of cancers, while they can behave as tumor suppressors in others. Therefore, studying the relation between the molecular clock and each particular type of cancer is fundamental to broaden the understanding of this illness and to improve cancer treatment outcomes.

\section{THE ROAD THAT LEADS FROM CIRCADIAN PHYSIOLOGY TO CHRONOPHARMACOLOGY.}

The circadian system determines temporal homeorhesis in physiology, set signals for both central and peripheral oscillators through SCN endocrine and autonomic outputs, as well as the activity-rest and feeding-fasting behavioral cycles. In consequence, there are rhythms in most hormones, cardiac output, blood flow and components, microvascular bed dynamics, endothelial barrier function, gastric ph, intestinal absorption, liver metabolism and biliary excretion, renal clearance, blood brain barrier permeability, etc ${ }^{55-58}$. Taking this into account, it is not surprising that the pharmacokinetic and pharmacodynamic properties of most drugs show daily oscillations, determining optimum times for administration that increase their therapeutic indexes.

\section{Chronopharmacokinetics}

Different rhythms in drug absorption, distribution, metabolism, and excretion (ADME) can be described. Drug concentrations in body compartments such as the blood and the target tissue are regulated by these processes, which can be used to determine the pharmacological effects of drugs. We will describe each process in more depth in the next sections.

\section{Absorption}

For orally administered drugs, the diurnal variation in gastric emptying, gastric $\mathrm{pH}$, motility and peristalsis, enzymes and drug transporters expressed in the gut, and ultimately in blood flow and perfusion to gastrointestinal tract, determines the rhythm in their absorption ${ }^{59}$. Oral bioavailability of drugs subjected to a significant first-pass effect is likely to show a temporal variation in plasma levels.

Goo et al. showed that increased gastric emptying and motility during the day, as compared to the night, results in increased absorption of lipophilic drugs when they are administered in the morning ${ }^{60}$. Several lipid transporters, such as microsomal triglyceride transfer protein (MTP), apolipoprotein B (ApoB), ApoA $4^{61}$, are controlled by the circadian clock, resulting in robust oscillations in lipid absorption from the intestine ${ }^{62}$. This circadian oscillation is related to the rhythm of absorption of some lipophilic drugs, such as nifedipine ${ }^{63}$. Rhythms in protein transporters were also described: some members of the ATP-binding cassette (ABC) transporter family (proteins important in transporting the drug from inside the cells back to the intestinal lumen), such as MDR1 and MCT1, showed circadian oscillations in their mRNAs ${ }^{64}$.

Blood flow and gastric $\mathrm{pH}$ are also fundamental for drug absorption. It has been described that blood flow peaks at the beginning of the day ${ }^{58}$, while the acidity of gastric juice peaks in the evening ${ }^{57}$. Since lipophilic drug absorption decreases with $\mathrm{pH}$, this generates an oscillation along the day ${ }^{10}$. 
Daily variations in blood flow determines not only differences in absorption, but also differential drug distribution and elimination. In addition, both drug solubility and route of administration may influence the circadian oscillation in drug absorption. Lipid-soluble drugs seem more prone to showing circadian temporal variations in pharmacokinetics than water-soluble drugs $^{65-67}$. The reason for this difference may be the oscillations in lipid receptors explained above. Of course, parenteral route may minimize these changes, which might be abolished when applying intravascular administration.

The multiplicity of all these factors influence the absorption of several drugs. It has been reported that the absorption of nitrates ${ }^{68}$, benzodiazepines ${ }^{69}$, drugs used to treat essential hypertension ${ }^{70}$, and antidepressants ${ }^{71}$, exhibits circadian rhythms. Information about some of these drugs taken the main physiological rhythm underlying their chronopharmacokinetic profile, and their efficient time for administration, is summarized in Table 1.

\section{Distribution}

Little is known regarding the circadian variation of drug distribution. Distribution from the vascular blood compartment is mainly affected by blood protein binding, blood flow, and capillary perfusion to a given tissue, all of which clearly show circadian variations ${ }^{72,73}$. Two hypotheses have been proposed: that the variation of drug binding to plasma proteins, and the variation in both vascular endothelial and target cell permeability, influences drug distribution. Angeli et al. described the capacity of prednisolone and cortisol to bind serum corticosteroid-binding globulin in humans, being maximum at midnight ${ }^{74}$. Kervezee and colleagues found that the concentration of morphine in the brain of rats depends on the time of day, showing a 12-hour rhythm, with the lowest values at the light-dark transitions, for the transport from brain to blood ${ }^{75}$.
Zhang et al. found that the permeability of the blood brain barrier in Drosophila varies with the time of day, being higher at night. This rhythm depends on a molecular clock in the perineurial glia ${ }^{55}$. Nakazato et al. found that bmall deficiency in mice disrupts the blood brain barrier integrity, suggesting that this clock protein plays a fundamental function in brain transport ${ }^{76}$. All these results suggest that the permeability of cell membranes has an important influence on the transport of many drugs, and that further studies should be performed to determine in which cases it is important to take this factor into account.

\section{Metabolism}

Since the liver represents the main organ involved in the metabolism of xenobiotics, it is fundamental to recognize which drug-metabolizing enzymes may have circadian activity. There are various enzymes that show diurnal oscillations in the liver, as for instance, tyrosine-Q-ketoglutarate transaminase ${ }^{77}$, steroid hydroxylase $^{78}$ and UDP-glucuronosyltransferases ${ }^{79}$. Hexobarbital oxidase and p-nitroanisole 0-demethylase were the first enzymes that demonstrated to have daily rhythms in drug metabolizing activity $^{80}$. After these, many other studies were performed, that enable to understand in more depth the circadian rhythmicity of drug metabolism. Miyazaki et al. described circadian oscillations in the Cytochrome P450s (Cyp) superfamily ${ }^{81}$, the principal enzymes for drug metabolism and detoxification ${ }^{82}$. Recently, Deng et al. found that the peroxisome proliferator-activated receptor (Ppar- $\gamma$ ) plays an important role in the circadian regulation of hepatic Cyp2a5, demonstrating for the first time that this nuclear receptor is a transcriptional activator of the cytochrome, and its rhythmic expression contributes to the circadian expression of Cyp2a $5^{83}$. Sulfotransferase $1 \mathrm{a} 1$ enzyme was also demonstrated to undergo circadian oscillations at both mRNA and protein levels, being regulated by BMAL1 ${ }^{84}$. The same was described for Flavincontaining monooxygenase 5 , confirming positive regulation of the enzyme by BMAL1 and REV-ERB $\alpha^{85}$.

Table 1. Efficacy of a morning versus evening schedule of certain drugs. The table summarizes examples of drugs that have been tested in a morning vs. evening schedule. The main rhythms that influence chronopharmacokinetic oscillations are listed.

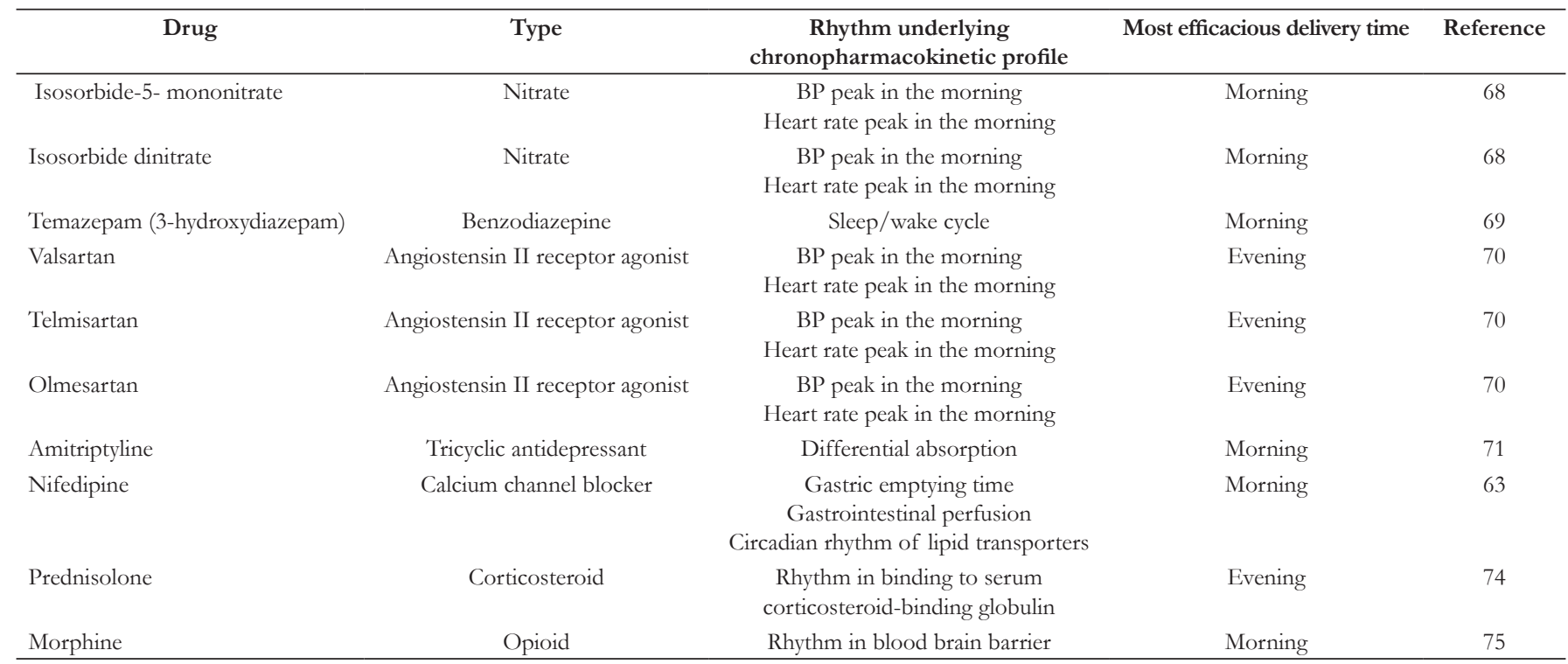


Pethidine, a synthetic narcotic analgesic, was also studied. Chengliang et al. studied the influence of dosing time on the chronopharmacokinetics of pethidine in mice. This drug is metabolized to norpethidine into the liver. They observed significant dosing-time dependence in the pharmacokinetics of pethidine and norpethidine, with a higher peak in serum drug concentrations (Cmax) during the dark phase. These results suggest that norpethidine is accumulating during the dark phase, causing toxicity and adverse reactions if not administered at the right time ${ }^{86}$.

\section{Excretion}

Diurnal rhythms have been described for glomerular filtration rate, effective renal plasma flow, tubular secretion, urine output, and urinary excretion of electrolytes ${ }^{87}$. These rhythms influence the differential excretion rates for drugs at different times of the day. As in the absorption phenomena, the excretion is determined by diurnal variations in systemic blood pressure, and in urinary $\mathrm{pH}$ determining hydrophilic ion trapping. The renin-angiotensin system and renal blood flow controlling glomerular filtration are also important factors. Particularly, urinary $\mathrm{pH}$ has been demonstrated to have a circadian rhythm, being lower during the night and higher in the day ${ }^{88}$.This effect may explain the diurnal variation in the excretion of amphetamines ${ }^{89}$.

Circadian modulation of biliary and fecal elimination also contributes to time-dependent excretion. Oh et al. reported a circadian rhythm in the expression of multidrug resistance-associated protein 2 (Mrp2), a major mediator of the hepatobiliary transport process that determines the systemic and liver exposure for numerous drugs ${ }^{90}$. These results help to explain the daily variation in liver toxicity and drug excretion mechanisms.

\section{Chronopharmacodynamics}

In addition to chronopharmacokinetics, circadian variations in the interaction of a drug with its target have been described, including the affinity of the drug for its target, the amount of the target present in a given tissue and the baseline activity of the target system ${ }^{10}$. Globally, rhythmic and temporally predictable alterations in either the susceptibility or the sensitivity of a target biological system to a drug are important for clinical treatment ${ }^{91}$. Examples of this are rhythms in receptor number or conformation, secondary messengers, metabolic pathways, and/or free-to-bound fraction of medications ${ }^{92,93}$. Taken as a whole, these effects determine the chronopharmacodynamics of a given drug.

Cardiovascular drug pharmacodynamics have been extensively studied, and there is a great amount of evidence related to the impact of circadian rhythms on their effects. Various $\beta$-blockers ${ }^{94}$, nitrates ${ }^{95}$, and calcium channel blockers ${ }^{96}$, were applied taking the time of day into account. Propranolol, a drug used for treating hypertension, is currently being applied with a chrono drug delivery system in the USA, and the peak of its effect was achieved when applied between 8 A.M. and 2 P.M ${ }^{97}$.
Given the rapid increase of blood pressure (BP) in the hours after awakening, the administration of propranolol previously to the BP increase helps hypertensive patients to prevent heart attacks more efficiently in the hours of the increased morning risk.

Verapamil, a calcium channel blocker, enalapril, an angiotensin-converting-enzyme inhibitor, and losartan, an antagonist of the angiotensin II receptor, are all used for the treatment of cardiovascular disease. Bakris et al. studied these three drugs on a chronopharmacological scheme of administration in patients, and found that verapamil was most effective in lowering morning systolic and diastolic BP than either enalapril or losartan. Thus, verapamil produces changes in $\mathrm{BP}$ and pulse that more closely match the normal circadian hemodynamic rhythms ${ }^{98}$. Several chronopharmacological studies were performed on other calcium channel blockers and, in general, their lowering effect of $\mathrm{BP}$ was found to be higher in the daytime ${ }^{94}$.

\section{CHRONOPHARMACOLOGY AND CHRONO- THERAPY IN CANCER TREATMENT.}

More than 30 anticancer drugs have been studied using circadian administration schedules, and it was found that their effectiveness vary by $50 \%$ or more according to the time of administration in mice or rats ${ }^{99}$. The vast majority of anticancer drug targets are molecules present in normal cells, so it is not surprising that these targets display circadian oscillations even in the transformed cells. More than 170 drug targets are controlled by the circadian clock $^{100}$. Drug pharmacokinetics can vary with the time of administration, as explained above. Nevertheless, cellular rhythms seem to be the key determinants of anticancer drug chronopharmacology, because they can modulate the generation or the catabolism of intracellular cytotoxic substances, their interactions with the molecular targets leading to cell dysfunction or death, and the repair of cytotoxic damage ${ }^{101}$. However, explaining the biology of cancer is complex, and, particularly, the circadian rhythms vary in each kind of tumor. To determine the correct dosing time for each anticancer drug, it is necessary to evaluate not only the chronopharmacokinetics and dynamics of the drug, but also the possibility of circadian oscillations in their targets, even if the tumor has a different circadian rhythm than normal cells. The chronopharmacological approach is fundamental in cancer, because it gives the opportunity to find the time of day when the toxicity is maximum in the tumoral cells, while decreased in normal cells, thereby reducing the adverse effects of chemotherapics.

\section{Time-of-day administration of drugs}

Many chemotherapeutic agents have been studied under a chronopharmacological approach. Gorbacheva et al. analyzed the circadian administration of cyclophosphamide. They found, in wild type mice, that the sensitivity to the drug varies with the time of day, being greater during the dark-light transition. However, clock and bmall knockouts show the same sensitivity to the drug in all the times of administration, suggesting that the response to cyclophosphamide is regulated by CLOCK/BMAL1 ${ }^{102}$. 
Slat et al. showed rhythms in apoptosis induced by Temozolomide in glioblastoma cell lines, and these rhythms were lost in bmal1 knockouts, suggesting that circadian sensitivity to Temozolomide is also regulated by bmal $1^{15}$. Oxaliplatin is maybe one of the most paradigmatic examples in cancer chronotherapies. In 1990, the drug was discarded to treat colorectal cancer because of its excessive toxicity, particularly in the liver and kidney ${ }^{103}$. In 1993, Levi et al. studied the therapeutic index of this drug in clinical phase I chronotherapy trial. This trial established the safety of the drug for patients, with a peak at 16:00 $\mathrm{h}$ in phase I (104) and $\mathrm{II}^{105}$ clinical trials. It appears that the problem was not the drug itself, but its time of administration, which, when optimized, improved effectiveness and limited toxicity.

In a mice model of Glasgow osteosarcoma, Granda et al. studied the chronotherapeutic effects of oxaliplatin and irinotecan, a topoisomerase I inhibitor, finding that both drugs lead to a decrease in tumor growth and an increase in estimated life span (maximum effect during the light phase for irinotecan and during the dark phase for oxaliplatin) ${ }^{106}$. Later, studies were conducted to analyze the variations in drug pharmacokinetics of both chemotherapeutics, in order to find the time of less toxicity. Irinotecan showed higher toxicity (leukopenia, body weight loss) when administered in the late night ${ }^{107}$. When applied during the night, oxaliplatin showed a $76 \%$ survival after administering a high dose, compared to $24 \%$ survival when administered during the day in mice ${ }^{108}$. Phase I and II trials were conducted also with irinotecan (in combination with folinic acid, 5- fluorouracil (5FU) and oxaliplatin ${ }^{109}$. Similar results were found in mice for other platinum complexes, such as cisplatin and carboplatin, where the toxic effects were half as severe when administered $16 \mathrm{~h}$ after light onset as compared to 8 hours after light onset ${ }^{110}$. In a recent study, Yang et al. found in mice that the repair of both transcribed and non transcribed strands of DNA occur at different circadian phases. While the repair of the transcribed strand is dictated by the phase of transcription of each gene, the repair of any non transcribed strand peaks in the middle of the light phase. As cisplatin is a drug that causes DNA damage, they hypothesized that future research on timed dosage of cisplatin could potentially reduce damage to healthy tissue if applied at the correct time, when the DNA repair system is active in normal tissue, but not active in tumor cells ${ }^{111}$. It was already shown that the nucleotide excision repair pathway is controlled by the circadian clock by the direct binding of BMAL1 to E-box sites in the promoter of $X p a^{112}$, a fundamental repair protein factor, resulting in circadian expression of this protein ${ }^{113}$.

Methotrexate and 6-mercaptopurine were also applied taking the time of day into account. Schmiegelo et al. studied morning versus evening administration in children with acute lymphoblastic leukemia. They found that applying the combination of drugs in the evening had a better probability of event-free survival than when administered in the morning ${ }^{114}$. Another drug that was widely studied is $5-\mathrm{FU}$. This drug is an antimetabolite of the fluoropyrimidine family, used to treat gastrointestinal cancer. It is probably one of the most studied anti-tumor drugs under the chronopharmacological scheme.
It has been shown that cells that are entering S-phase undergoing DNA synthesis are more susceptible to $5-\mathrm{FU}^{87}$. Particularly, this drug is rhythmically catabolized by dehydropyrimidine dehydrogenase (DPD $)^{115}$. The active metabolites that are formed suppress DNA synthesis through inhibition of thymidilate synthase (TS), an enzyme that shows rhythmic activity ${ }^{116}$. These circadian variations in pharmacokinetics determine the effect of the drug, being more tolerable if it is given during the light phase for mice and rats, and having a maximal toxicity inversely correlated with DPD activity ${ }^{117}$.

Clinical studies have shown that, if administered by continuous intravenous infusion, 5-FU serum levels fluctuate, with higher levels at night ${ }^{12}$. A phase III trial was conducted with 5-FU, folinic acid and oxaliplatin, treating one group of patients with colorectal cancer with a chronomodulated scheme, whereas the other one was treated in a constant manner for 5 days. The chronomodulated group showed a better objective response (53\% versus $32 \%$ ) and higher median survivals (19 months versus 14.9 months). This trial showed that if drug delivery was chronomodulated rather than constant over time, the drugs were more effective and less toxic ${ }^{118}$. The better tolerability of $5-\mathrm{FU}$ in humans can be explained because of both the higher levels of DPD activity in circulating lymphocytes at night, and the lower number of cells in S-phase in human bone marrow, oral mucosa, and skin $^{119}$. Then, it can be stated that circadian differences in the toxicity of 5-FU can occur because of the diurnal regulation in enzymes involved in its metabolism, resulting in a higher toleration of $5-\mathrm{FU}$ when thymidylate synthase activity is low and DPD activity is highest ${ }^{10}$.

Similar results were obtained for fluorodeoxyuridine (FUDR), another fluoropyrimidine: when applied in a chronopharmacological scheme, the drug showed less toxicity than when given constantly to patients with metastasic renal cancer ${ }^{13}$. Focan et al. conducted a randomized study with intravenous 5-FU and intrahepatic FUDR in patients with liver metastases from colorectal cancer. One group of patients received a constant infusion, whereas the other one received a circadian infusion. Toxicity (alopecia, neutropenia and skin) was fewer, and median survival higher, in patients receiving the circadian scheme, in spite of receiving a higher dose $\mathrm{e}^{120}$. Some studies were conducted to treat advanced lung carcinoma with 5-FU. Focan et al. applied a chronomodulated 5-day venous infusion of the combination of 5-FU, carboplatin and folinic acid to patients. Toxicity (mucositis, diarrhea, alopecia) was found only in 3\% of the patients, improving tolerance and quality of life ${ }^{121}$. Nevertheless, the current treatment of choice for advanced lung carcinoma is Erlotinib, an epidermal growth factor receptor (EGFR) inhibitor. A study was conducted where this drug was given to mice at different times of day, demonstrating a significant reduction in tumor growth when giving the drug during the light phase $\mathrm{e}^{122}$.

Table 2 summarizes the drugs mentioned in this section that were assessed in phase I and II trials, with its most efficacious delivery time. 
Table 2. Efficacy of a morning versus evening schedule of certain anti-cancer drugs. A short list of anti-cancer drugs assessed in phase I and II clinical trials, with the cancer types for which they were indicated. "Chronomulated infusion" indicates that the trial consisted in comparing a constant vs. a chrono-modulated infusion, with a better outcome for the latter.

\begin{tabular}{cccc}
\hline Drug & Type of cancer assessed & Most efficacious delivery time & Reference \\
\hline Oxaliplatin & Colorectal, lung & Afternoon/Evening & $104-106,124$ \\
Methotrexate & Acute lymphoblastic leukemia. & Evening & 114 \\
6-mercaptopurine & Acute lymphoblastic leukemia. & Evening & 114 \\
5-fluorouracil & Colorectal, liver metastases, lung & Morning & $109,119,121,124,125$ \\
Fluorodeoxyuridine & Renal cancer & Chronomodulated infusion & 117 \\
Folinic acid & Colorectal cancer & Chronomodulated infusion & $118,121,124$ \\
Irinotecan & Colorectal & Chronomodulated infusion & $106,107,109,123,124$ \\
Cisplatin & Melanoma & Evening & $110,112,127$ \\
Paclitaxel & Chead and neck squamous cell carcinoma & 125 \\
Capecitabine & Rectal cancer & Morning & 126 \\
Citrovorum & Nasopharyngeal carcinoma & Evening & 127 \\
Cetuximab & Colorectal liver metastases & Chronomodulated infusion & 128 \\
\hline
\end{tabular}

Finally, it is important to also mention chronoradiotherapy. Indeed, most tumors are treated with a combination of surgical removal, chemotherapy and radiotherapy. Several studies have investigated the outcome of radiotherapy treatment at different times of the day for treating rectal cancer, head and neck cancer and brain metastasis, among others $^{129-131}$. Noh et al. treated patients with breast cancer in different times of the day, showing that the early morning treated group experienced less acute skin reactions compared to the late morning group ${ }^{132}$. Another study treated 67 women with cervical cancer with radiotherapy, discovering that there was a higher incidence of severe hematological toxicity between $21 \mathrm{~h}$ and $23 \mathrm{~h}^{133}$. It has been already shown that per 2 deficient mice display less radiosensitivity to gamma radiation treating tumor growth, because of downregulation of tumor suppressor genes, such as Mdm- $2^{45}$. Recently, Zhu et al. showed that glioma cells with low expression of PER1 displayed low radiosensitivity, with resulted in minor DNA damage when exposed to X-ray irradiation. This effect may be due to the disminution of p53 in the cells that have low PER1 expression. Thus, if there is less $\mathrm{p} 53$, there will be a reduced effect of chk2-p53 pathway, leading to less irradiated cells entering apoptosis ${ }^{134}$. One of the secondary effects of radiotherapy is hair loss. Plikus et al. described the circadian clock machinery in mice hair, with maximum hair growth in the morning and maximum hair repair in the evening ${ }^{135}$. Taking this into account, they found that hair loss upon exposure to gamma radiation was higher in the morning, when there are more cells undergoing mitosis. Cry1/2-/- mice did not exhibit rhythmic radioprotective effect, demonstrating that this effect is regulated by the circadian clock.

\section{Targeting the circadian clock}

One way to improve cancer therapeutics is to directly target the circadian clock. Depending on the type of tumor, the inhibition of tumor growth can be achieved by enhancing or suppressing circadian rhythmicity. Kiessling et al. found that less tumor growth resulted in allogeneic transplant when enhancing circadian clock function using dexamethasone, forskolin, or heat shock in B16 melanoma cells ${ }^{43}$.
Another study, conducted in non-tumorigenic MCF10A and tumorigenic breast cancer MDA-MB-31 bmal1 knockout cells, showed opposite effects. In MCF10A, the lack of bmal1 resulted in an increased sensitivity to the genotoxic agents cisplatin and doxorubicin, whereas in MDA-MB-31 it induced a more invasive capacity ${ }^{50}$. Thus, in this type of breast cancer cells, an overexpression of BMAL1 could lead to a reduced tumor phenotype. Weiliang et al. found a similar result in pancreatic cells: disturbing bmal1 led to tumorigenesis and invasion ${ }^{136}$. This result suggests that in pancreatic cells, bmall acts as a tumor suppressor gene. Further studies should be conducted in pancreatic cancer cells to determine if an enhancement of the BMAL1 pathway leads to a suppression of the tumoral phenotype. Finally, Wagner et al. studied the effect of SR9009 ${ }^{137}$, a REV-ERB $\alpha$ agonist, in T98G glioblastoma cell line. The cells showed a cell cycle arrest, affected tumor metabolism and cytotoxic effects ${ }^{138}$, suggesting that targeting directly the circadian clock is an interesting strategy for glioblastoma treatment.

Recent work in human osteosarcoma cell lines has shown that circadian periods of bmal1 and per 2 can be modulated using certain small molecules as direct (such as KL001, which targets are CRY and $\mathrm{CKI} \delta / \varepsilon$ ) or indirect (such as SP600125 and Chir99021, which targets are JNK, GSK3 $\beta$, and TOPII) circadian modulators. SP600125 showed a lengthening of bmal1 period, KL001 lengthened both per1 and bmal1 periods, whereas Chir99021 shortened bmal1 period. KL001 directly binds to CRY stabilizing it. When this molecule was assessed in human osteosarcoma cells, it was demonstrated that reduces migration and proliferation, thus, inhibiting tumor growth and invasion ${ }^{139}$.

\section{CONCLUSIONS}

In this article, we clearly stated the importance of circadian rhythms in cancer treatment. The recent findings that we have highlighted help to understand in more depth the growing field of chronopharmacology. Available chemotherapies have several limitations, including resistance due to polymorphisms for xenobiotic metabolism, among other factors, or due to deleterious effects in normal tissues. Indeed, 
to find a circadian time window improving drug efficacy is of key importance. In addition, chrono-chemotherapy also has limitations, as most drugs administered chronically may achieve saturating steady-state concentrations overpassing circadian pharmacokinetic and dynamic variations. Nevertheless, there is still a long way to go. Most of the circadian expression data at the genomic level are based only on rodent models, whereby it is necessary to improve and broaden the little data available on humans. On the other hand, few cancer drugs are currently applied under a chronopharmacological approach, despite the growing pre-clinical and clinical evidence. Furthermore, if both chemotherapy and radiotherapy can be optimized by time of day administration, it would be a fundamental advance for the quality of life and overall survival of patients. It is necessary to generate bonds with public health institutions, medical staff, health personnel, clinical trial nodes and pharmaceutical companies, so that all these evidences begin to be applied more frequently in the clinic.

\section{ACKNOWLEDGMENTS}

Special thanks to Universidad Nacional de Quilmes, Consejo Nacional de Investigaciones Científicas y Técnicas (CONICET) and Agencia Nacional de Promoción de la Ciencia y la Tecnología (ANPCy'T) for financial support.

\section{REFERENCES}

1. Golombek DA, Rosenstein RE. Physiology of Circadian Entrainment. Physiol Rev. 2010.

2. Gillette MU, Medanic M, McArthur AJ, Liu C, Ding JM, Faiman LE, et al. Intrinsic neuronal rhythms in the suprachiasmatic nuclei and their adjustment. Ciba Foundation symposium. 1995.

3. Welsh DK, Yoo SH, Liu AC, Takahashi JS, Kay SA. Bioluminescence imaging of individual fibroblasts reveals persistent, independently phased circadian rhythms of clock gene expression. Curr Biol. 2004.

4. Schibler U, Gotic I, Saini C, Gos P, Curie T, Emmenegger Y, et al. Clock-Talk: Interactions between central and peripheral circadian oscillators in mammals. In: Cold Spring Harbor Symposia on Quantitative Biology. 2016.

5. Motohashi H, Sukigara H, Tahara Y, Saito K, Yamazaki M, Shiraishi T, et al. Polyporus and Bupleuri radix effectively alter peripheral circadian clock phase acutely in male mice. Nutr Res. 2017.

6. Kamagata M, Ikeda Y, Sasaki H, Hattori Y, Yasuda S, Iwami S, et al. Potent synchronization of peripheral circadian clocks by glucocorticoid injections in PER2::LUC-Clock/Clock mice. Chronobiol Int. 2017.

7. Damiola F, Le Minli N, Preitner N, Kornmann B, Fleury-Olela F, Schibler $\mathrm{U}$. Restricted feeding uncouples circadian oscillators in peripheral tissues from the central pacemaker in the suprachiasmatic nucleus. Genes Dev. 2000.

8. Stokkan KA, Yamazaki S, Tei H, Sakaki Y, Menaker M. Entrainment of the circadian clock in the liver by feeding. Science (80- ). 2001.

9. Y. T, S. S. Chronobiology and nutrition. Neuroscience. 2013.

10. Paschos GK, Baggs JE, Hogenesch JB, FitzGerald GA. The Role of Clock Genes in Pharmacology. Annu Rev Pharmacol Toxicol. 2010.

11. Bjarnason GA. Chronobiology implications for cancer chemotherapy. Acta Oncol (Madr). 1995.

12. Bressolle F, Joulia JM, Pinguet F, Ychou M, Astre C, Duffour J, et al. Circadian rhythm of 5-fluorouracil population pharmacokinetics in patients with metastatic colorectal cancer. Cancer Chemother Pharmacol. 1999.

13. Roemeling R V., Hrushesky WJ. Circadian pattern of continuous FUDR infusion reduces toxicities. Prog Clin Biol Res. 1987.

14. Gutiérrez-Monreal MA, Treviño V, Moreno-Cuevas JE, Scott SP. Identification of circadian-related gene expression profiles in entrained breast cancer cell lines. Chronobiol Int. 2016.

15. Slat EA, Sponagel J, Marpegan L, Simon T, Kfoury N, Kim A, et al. Cell-intrinsic, Bmal1-dependent Circadian Regulation of Temozolomide Sensitivity in Glioblastoma. J Biol Rhythms. 2017.
16. Matsuo T, Yamaguchi S, Mitsui S, Emi A, Shimoda F, Okamura H. Control mechanism of the circadian clock for timing of cell division in vivo. Science (80-). 2003.

17. Gery S, Komatsu N, Baldjyan L, Yu A, Koo D, Koeffler HP. The Circadian Gene Per1 Plays an Important Role in Cell Growth and DNA Damage Control in Human Cancer Cells. Mol Cell. 2006.

18. Lowrey PL, Takahashi JS. MAMMALIAN CIRCADIAN BIOLOGY: Elucidating Genome-Wide Levels of Temporal Organization. Annu Rev Genomics Hum Genet. 2004.

19. Takahashi JS, Hong HK, Ko CH, McDearmon EL. The genetics of mammalian circadian order and disorder: Implications for physiology and disease. Nature Reviews Genetics. 2008.

20. Preitner N, Damiola F, Luis-Lopez-Molina, Zakany J, Duboule D, Albrecht U, et al. The orphan nuclear receptor REV-ERB $\alpha$ controls circadian transcription within the positive limb of the mammalian circadian oscillator. Cell. 2002.

21. Sato TK, Panda S, Miraglia LJ, Reyes TM, Rudic RD, McNamara P, et al. A functional genomics strategy reveals rora as a component of the mammalian circadian clock. Neuron. 2004.

22. Gallego M, Virshup DM. Post-translational modifications regulate the ticking of the circadian clock. Nature Reviews Molecular Cell Biology. 2007.

23. Wu M, Zeng J, Chen Y, Zeng Z, Zhang J, Cai Y, et al. Experimental chronic jet lag promotes growth and lung metastasis of Lewis lung carcinoma in C57BL/ 6 mice. Oncol Rep. 2012.

24. Filipski E, Delaunay F, King VM, Wu MW, Claustrat B, Gréchez-Cassiau A, et al. Effects of chronic jet lag on tumor progression in mice. Cancer Res. 2004

25. Logan RW, Zhang C, Murugan S, O'Connell S, Levitt D, Rosenwasser AM, et al. Chronic Shift-Lag Alters the Circadian Clock of NK Cells and Promotes Lung Cancer Growth in Rats. J Immunol. 2012.

26. Schernhammer ES, Laden F, Speizer FE, Willet WC, Hunter DJ, Kawachi I, et al. Night-shift work and risk of colorectal cancer in the Nurses' Health Study. J Natl Cancer Inst. 2003.

27. Wolff G, Duncan MJ, Esser KA. Chronic phase advance alters circadian physiological rhythms and peripheral molecular clocks. J Appl Physiol. 2013.

28. Yu Y, Li Y, Zhou L, Yang G, Wang M, Hong Y. Cryptochrome 2 (CRY2) suppresses proliferation and migration and regulates clock gene network in osteosarcoma cells. Med Sci Monit. 2018.

29. Lee Y, Lahens NF, Zhang S, Bedont J, Field JM, Sehgal A. G1/S cell cycle regulators mediate effects of circadian dysregulation on tumor growth and provide targets for timed anticancer treatment. PLoS Biol. 2019.

30. Chun SK, Chung S, Kim HD, Lee JH, Jang J, Kim J, et al. A synthetic cryptochrome inhibitor induces anti-proliferative effects and increases chemosensitivity in human breast cancer cells. Biochem Biophys Res Commun. 2015.

31. Lellupitiyage Don SS, Lin HH, Furtado JJ, Qraitem M, Taylor SR, Farkas ME. Circadian oscillations persist in low malignancy breast cancer cells. Cell Cycle. 2019.

32. Dulong S, Ballesta A, Okyar A, Levi F. Identification of circadian determinants of cancer chronotherapy through In Vitro chronopharmacology and mathematical modeling. Mol Cancer Ther. 2015.

33. Fu XJ, Li HX, Yang K, Chen D, Tang H. The important tumor suppressor role of PER1 in regulating the cyclin-CDK-CKI network in SCC15 human oral squamous cell carcinoma cells. Onco Targets Ther. 2016.

34. Gery S, Gombart AF, Yi WS, Koeffler C, Hofmann WK, Koeffler HP. Transcription profiling of C/EBP targets identifies Per2 as a gene implicated in myeloid leukemia. Blood. 2005.

35. Zhang Y, Giacchetti S, Parouchev A, Hadadi E, Li X, Dallmann R, et al. Dosing time dependent in vitro pharmacodynamics of Everolimus despite a defective circadian clock. Cell Cycle. 2018.

36. Farshadi E, Yan J, Leclere P, Goldbeter A, Chaves I, van der Horst GTJ The positive circadian regulators CLOCK and BMAL1 control G2/M cell cycle transition through Cyclin B1. Cell Cycle. 2019.

37. Gréchez-Cassiau A, Rayet B, Guillaumond F, Teboul M, Delaunay F. The circadian clock component BMAL1 is a critical regulator of p21 WAF1/ CIP1 expression and hepatocyte proliferation. J Biol Chem. 2008.

38. Chen ST, Choo KB, Hou MF, Yeh KT, Kuo SJ, Chang JG. Deregulated expression of the PER1, PER2 and PER3 genes in breast cancers. Carcinogenesis. 2005.

39. Zhao H, Zeng ZL, Yang J, Jin Y, Qiu MZ, Hu XY, et al. Prognostic relevance of Period1 (Per1) and Period2 (Per2) expression in human gastric cancer. Int J Clin Exp Pathol. 2014.

40. Hsu CM, Lin SF, Lu CT, Lin PM, Yang MY. Altered expression of circadian clock genes in head and neck squamous cell carcinoma. Tumor Biol. 2012. 
41. Wang Q, Ao Y, Yang K, Tang H, Chen D. Circadian clock gene Per2 plays an important role in cell proliferation,apoptosis and cell cycle progression in human oral squamous cell carcinoma. Oncol Rep. 2016.

42. Luo Y, Wang F, Chen LA, Chen XW, Chen ZJ, Liu PF, et al. Deregulated expression of Cry1 and Cry2 in human gliomas. Asian Pacific J Cancer Prev. 2012.

43. Kiessling S, Beaulieu-Laroche L, Blum ID, Landgraf D, Welsh DK, Storch KF, et al. Enhancing circadian clock function in cancer cells inhibits tumor growth. BMC Biol. 2017.

44. Katamune C, Koyanagi S, Shiromizu S, Matsunaga N, Shimba S, Shibata $\mathrm{S}$, et al. Different roles of negative and positive components of the circadian clock in oncogene-induced neoplastic transformation. J Biol Chem. 2016.

45. Fu L, Pelicano H, Liu J, Huang P, Lee CC. The circadian gene Period2 plays an important role in tumor suppression and DNA damage response in vivo. Cell. 2002.

46. Papagiannakopoulos T, Bauer MR, Davidson SM, Heimann M, Subbaraj L, Bhutkar A, et al. Circadian Rhythm Disruption Promotes Lung Tumorigenesis. Cell Metab. 2016.

47. Hernández-Rosas F, Santiago-García J. Ritmos circadianos, genes reloj y cáncer. Arch Med. 2010.

48. Gotoh T, Vila-Caballer M, Santos CS, Liu J, Yang J, Finkielstein C V. The circadian factor Period 2 modulates p 53 stability and transcriptional activity in unstressed cells. Mol Biol Cell. 2014.

49. Jiang W, Zhao S, Jiang X, Zhang E, Hu G, Hu B, et al. The circadian clock gene Bmal1 acts as a potential anti-oncogene in pancreatic cancer by activating the p53 tumor suppressor pathway. Cancer Lett. 2016.

50. Korkmaz T, Aygenli F, Emisoglu H, Ozcelik G, Canturk A, Yilmaz S, et al. Opposite Carcinogenic Effects of Circadian Clock Gene BMAL1. Sci Rep. 2018.

51. Kettner NM, Voicu H, Finegold MJ, Coarfa C, Sreekumar A, Putluri $\mathrm{N}$, et al. Circadian Homeostasis of Liver Metabolism Suppresses Hepatocarcinogenesis. Cancer Cell. 2016.

52. Yaping W, Sun N, Lu C, Bei Y, Qian R, Hua L. Upregulation of circadian gene "hClock" contribution to metastasis of colorectal cancer. Int J Oncol. 2017.

53. Ma T jiao, Zhang $Z$ wei, Lu Y lu, Zhang $Y$ ying, Tao D chang, Liu $Y$ qiang, et al. CLOCK and BMAL1 stabilize and activate RHOA to promote F-actin formation in cancer cells. Exp Mol Med. 2018.

54. El-Athman R, Genov NN, Mazuch J, Zhang K, Yu Y, Fuhr L, et al. The Ink4a/Arf locus operates as a regulator of the circadian clock modulating RAS activity. PLoS Biol. 2017.

55. Zhang SL, Yue Z, Arnold DM, Artiushin G, Sehgal A. A Circadian Clock in the Blood-Brain Barrier Regulates Xenobiotic Efflux. Cell. 2018.

56. Abbott NJ, Patabendige AAK, Dolman DEM, Yusof SR, Begley DJ. Structure and function of the blood-brain barrier. Neurobiology of Disease. 2010.

57. Moore JG, Englert E. Circadian rhythm of gastric acid secretion in man. Nature. 1970.

58. Lemmer B, Nold G. Circadian changes in estimated hepatic blood flow in healthy subjects. Br J Clin Pharmacol. 1991.

59. Labrecque G, Bélanger PM. Biological rhythms in the absorption, distribution, metabolism and excretion of drugs. Pharmacology and Therapeutics. 1991.

60. Goo RH, Moore JG, Greenberg E, Alazraki NP. Circadian variation in gastric emptying of meals in humans. Gastroenterology. 1987.

61. Sukumaran S, Almon RR, DuBois DC, Jusko WJ. Circadian rhythms in gene expression: Relationship to physiology, disease, drug disposition and drug action. Advanced Drug Delivery Reviews. 2010.

62. Pan X, Hussain MM. Clock is important for food and circadian regulation of macronutrient absorption in mice. J Lipid Res. 2009.

63. Bruguerolle B. Chronopharmacokinetics: Current status. Clinical Pharmacokinetics. 1998.

64. Stearns AT, Balakrishnan A, Rhoads DB, Ashley SW, Tavakkolizadeh A. Diurnal rhythmicity in the transcription of jejunal drug transporters. J Pharmacol Sci. 2008.

65. Langner B, Lemmer B. Circadian changes in the pharmacokinetics and cardiovascular effects of oral propranolol in healthy subjects. Eur J Clin Pharmacol. 1988.

66. Shiga T, Fujimura A, Tateishi T, Ohashi K, Ebihara A. Differences of Chronopharmacokinetic Profiles Between Propranolol and Atenolol in Hypertensive Subjects. J Clin Pharmacol. 1993.

67. Yoshiyama Y, Nakano S, Ohdo S, Ogawa N. Chronopharmacological Study of Valproic Acid in Mice: Comparison of Oral and Rectal Administration. J Pharmacobiodyn. 1992.

68. Scheidel B, Lemmer B. Chronopharmacology of oral nitrates in healthy subjects. Chronobiol Int. 1991.
69. Müller FO, Van Dyk M, Hundt HKL, Joubert AL, Luus HG, Groenewoud $\mathrm{G}$, et al. Pharmacokinetics of temazepam after day-time and night-time oral administration. Eur J Clin Pharmacol. 1987.

70. Hermida RC, Ayala DE, Mojón A, Fernández JR. Influence of circadian time of hypertension treatment on cardiovascular risk: Results of the MAPEC study. Chronobiol Int. 2010.

71. Nakano S, Hollister LE. Chronopharmacology of amitriptyline. Clin Pharmacol Ther. 1983.

72. Hawkins BT, Davis TP. The blood-brain barrier/neurovascular unit in health and disease. Pharmacological Reviews. 2005.

73. Wauschkuhn CA, Witte K, Gorbey S, Lemmer B, Schilling L. Circadian periodicity of cerebral blood flow revealed by laser-Doppler flowmetry in awake rats: Relation to blood pressure and activity. Am J Physiol - Hear Circ Physiol. 2005

74. Angeli A, Frajria R, De Paoli R, Fonzo D, Ceresa F. Diurnal variation of prednisolone binding to serum corticosteroid-binding globulin in man. Clin Pharmacol Ther. 1978.

75. Kervezee L, Hartman R, van den Berg DJ, Meijer JH, de Lange ECM. Diurnal variation in the pharmacokinetics and brain distribution of morphine and its major metabolite. Eur J Pharm Sci. 2017.

76. Nakazato R, Kawabe K, Yamada D, Ikeno S, Mieda M, Shimba S, et al. Disruption of Bmal1 impairs blood-brain barrier integrity via pericyte dysfunction. J Neurosci. 2017.

77. Civen M, Ulrich R, Trimmer BM, Brown CB. Circadian rhythms of liver enzymes and their relationship to enzyme induction. Science (80- ). 1967;

78. Colás A, Gregonis D, Moir N. Daily rhythms in the hydroxylation of 3-beta-hydroxyandrost-5-en-17-one by rat liver microsomes. Endocrinology. 1969.

79. Zhang T, Guo L, Yu F, Chen M, Wu B. The nuclear receptor Rev-erb $\alpha$ participates in circadian regulation of Ugt $2 \mathrm{~b}$ enzymes in mice. Biochem Pharmacol. 2019.

80. Nair V, Casper R. The influence of light on daily rhythm in hepatic drug metabolizing enzymes in rat. Life Sci. 1969.

81. Belanger PM, Lalande M. Day-night variations in the activity and composition of the microsomal mixed function oxidase of rat liver. Annu Rev Chronopharmacol. 1988.

82. Guengerich FP. Cytochrome P450 and chemical toxicology. Chemical Research in Toxicology. 2008

83. Deng J, Guo L, Wu B. Circadian regulation of hepatic cytochrome P450 $2 \mathrm{a} 5$ by peroxisome proliferator-activated receptor $\gamma$. Drug Metab Dispos. 2018.

84. Guo L, Yu F, Zhang T, Wu B. The clock protein BMAL1 regulates circadian expression and activity of sulfotransferase 1A1 in mice. Drug Metab Dispos. 2018.

85. Chen M, Guan B, Xu H, Yu F, Zhang T, Wu B. The molecular mechanism regulating diurnal rhythm of flavin-containing monooxygenase 5 in mouse liver. Drug Metab Dispos. 2019.

86. Zhang C, Yu Z, Li X, Xu Y, Liu D. Chronopharmacodynamics and chronopharmacokinetics of pethidine in mice. PLoS One. 2014.

87. Levi F, Schibler U. Circadian Rhythms: Mechanisms and Therapeutic Implications. Annu Rev Pharmacol Toxicol. 2007.

88. Koike M, Norikura R, Mizojiri K, Sugeno K. Time-dependent elimination of cinoxacin in rats. J Pharm Sci. 1984.

89. Musiek ES, FitzGerald GA. Molecular Clocks in Pharmacology. In 2013

90. Oh JH, Lee JH, Han DH, Cho S, Lee YJ. Circadian Clock Is Involved in Regulation of Hepatobiliary Transport Mediated by Multidrug Resistance-Associated Protein 2. J Pharm Sci. 2017.

91. Erkekoglu P, Baydar T. Chronopharmacodynamics of drugs in toxicological aspects: A short review for clinical pharmacists and pharmacy practitioners. J Res Pharm Pract. 2012.

92. Ohdo S, Koyanagi S, Matsunaga N, Hamdan A. Molecular basis of chronopharmaceutics. Journal of Pharmaceutical Sciences. 2011.

93. Hogenesch JB, Herzog ED. Intracellular and intercellular processes determine robustness of the circadian clock. FEBS Letters. 2011.

94. White WB, LaRocca GM. Chronopharmacology of cardiovascular therapy. Blood Pressure Monitoring. 2002.

95. Bode-Böger SM, Kojda G. Organic nitrates in cardiovascular disease. Cellular and Molecular Biology. 2005.

96. AV S. Calcium channel blockers. The jury is still out on whether they cause heart attacks and suicide. BMJ. 1998;316(1471-3).

97. LEMMER B, SCHEIDEL B, BEHNE S. Chronopharmacokinetics and Chronopharmacodynamics of Cardiovascular Active Drugs: Propranolol, Organic Nitrates, Nifedipine. Ann N Y Acad Sci. 1991.

98. Bakris G, Sica D, Ram V, Fagan T, Vaitkus PT, Anders RJ. A comparative trial of controlled-onset, extended-release verapamil, enalapril, and losartan on blood pressure and heart rate changes. Am J Hypertens. 2002. 
99. Lévi F. Chronotherapy for gastrointestinal cancers. Current Opinion in Oncology. 1996.

100. Zhang R, Lahens NF, Ballance HI, Hughes ME, Hogenesch JB. A circadian gene expression atlas in mammals: Implications for biology and medicine. Proc Natl Acad Sci U S A. 2014.

101. Lévi F. Circadian chronotherapy for human cancers. Lancet Oncology. 2001.

102. Gorbacheva VY, Kondratov R V., Zhang R, Cherukuri S, Gudkov A V., Takahashi JS, et al. Circadian sensitivity to the chemotherapeutic agent cyclophosphamide depends on the functional status of the CLOCK/ BMAL1 transactivation complex. Proc Natl Acad Sci U S A. 2005.

103. Cederroth CR, Albrecht U, Bass J, Brown SA, Dyhrfjeld-Johnsen J, Gachon F, et al. Medicine in the Fourth Dimension. Cell Metabolism. 2019.

104. Caussanel JP, Lévi F, Brienza S, Misset JL, Itzhaki M, Adam R, et al. Phase I trial of 5-day continuous venous infusion of oxaliplatin at circadian rhythm-modulated rate compared with constant rate. J Natl Cancer Inst. 1990.

105. Levi F, Perpoint B, Garufi C, Focan C, Chollet P, Depres-Brummer P, et al. Oxaliplatin activity against metastatic colorectal cancer. A phase II study of 5-day continuous venous infusion at circadian rhythm modulated rate. Eur J Cancer. 1993.

106. Granda TG, D’Attino RM, Filipski E, Vrignaud P, Garufi C, Terzoli E, et al. Circadian optimisation of irinotecan and oxaliplatin efficacy in mice with Glasgow osteosarcoma. Br J Cancer. 2002.

107. Ohdo S, Makinosumi T, Ishizaki T, Yukawa E, Higuchi S, Nakano S, et al. Cell cycle-dependent chronotoxicity of irinotecan hydrochloride in mice. J Pharmacol Exp Ther. 1997.

108. Boughattas NA, Lévi F, Roulon A, Reinberg A, Mathé G, Fournier C, et al. Orcadian Rhythm in Toxicities and Tissue Uptake of 1,2-Diamminocyclohexane(trans-1)oxalatoplatinum(II) in Mice. Cancer Res. 1989.

109. Focan C, Kreutz F, Graas MP, Longrée L, Focan-Henrard D, Demolin G, et al. Phase I - II study to assess the feasibility and activity of the triple combination of 5-fluorouracil/folinic acid, carboplatin and irinotecan (CPT-11) administered by chronomodulated infusion for the treatment of advanced colorectal cancer. Final report. Pathol Biol. 2013.

110. Boughattas NA, Levi F, Fournier C, Hecquet B, Lemaigre G, Roulon A, et al. Stable circadian mechanisms of toxicity of two platinum analogs (cisplatin and carboplatin) despite repeated dosages in mice. J Pharmacol Exp Ther. 1990.

111. Yang Y, Adebali O, Wu G, Selby CP, Chiou YY, Rashid N, et al. CisplatinDNA adduct repair of transcribed genes is controlled by two circadian programs in mouse tissues. Proc Natl Acad Sci U S A. 2018.

112. Dakup PP, Porter KI, Little AA, Gajula RP, Zhang H, Skornyakov E, et al. The circadian clock regulates cisplatin-induced toxicity and tumor regression in melanoma mouse and human models. Oncotarget. 2018.

113. Kang TH, Lindsey-Boltz LA, Reardon JT, Sancar A. Circadian control of XPA and excision repair of cisplatin-DNA damage by cryptochrome and HERC2 ubiquitin ligase. Proc Natl Acad Sci U S A. 2010.

114. Schmiegelow K, Glomstein A, Kristinsson J, Salmi T, Schrøder H, Björk O. Impact of morning versus evening schedule for oral methotrexate and 6-mercaptopurine on relapse risk for children with acute lymphoblastic leukemia. Am J Pediatr Hematol Oncol. 1997.

115. Daher GC, Zhang R, Soong SJ, Diasio RB. Circadian variation of fluoropyrimidine catablic enzymes in rat liver: Possible relevance to 5-fluorodeoxyuridine chemotherapy. Drug Metab Dispos. 1991.

116. Lincoln DW, Hrushesky WJM, Wood PA. Circadian organization of thymidylate synthase activity in normal tissues: A possible basis for 5-fluorouracil chronotherapeutic advantage. Int J Cancer. 2000.

117. Zhang R, Diasio RB, Diasio RB. Relationship Between CircadianDependent Toxicity of 5-Fluorodeoxyuridine and Circadian Rhythms of Pyrimidine Enzymes: Possible Relevance to Fluoropyrimidine Chemotherapy. Cancer Res. 1993.

118. Lévi FA, Zidani R, Vannetzel JM, Perpoint B, Focan C, Faggiuolo R, et al. Chronomodulated versus fixed-infusion - rate delivery of ambulatory chemotherapy with oxaliplatin, fluorouracil, and folinic acid (leucovorin) in patients with colorectal cancer metastases: A randomized multiinstitutional trial. J Natl Cancer Inst. 1994.

119. Bjarnason GA, Jordan RCK, Wood PA, Li Q, Lincoln DW, Sothern RB, et al. Circadian expression of clock genes in human oral mucosa and skin: Association with specific cell-cycle phases. Am J Pathol. 2001.
120. Focan C, Levi F, Kreutz F, Focan-Henrard D, Lobelle JP, Adam $\mathrm{R}$, et al. Continuous delivery of venous 5 -fluorpuracil and arterial 5-fluorodeoxyuridine for hepatic métastases from colorectal cancer: Feasibility and tolerance in a randomized phase II trial comparing flat versus chronomodulated infusion. Anticancer Drugs. 1999.

121. Focan C, Denis B, Kreutz F, Focan-Henrard D, Levi F. Ambulatory chronotherapy with 5-fluorouracil, folinic acid, and carboplatin for advanced non-small cell lung cancer. A phase II feasibility trial. J Infus Chemother. 1995.

122. Wang $\mathrm{P}$, An F, Zhuang $\mathrm{X}$, Liu J, Zhao L, Zhang B, et al. Chronopharmacology and mechanism of antitumor effect of erlotinib in Lewis tumor-bearing mice. PLoS One. 2014.

123. Giacchetti S, Cure H, Adenis A, Tubiana N, Vernillet L, ChedoubaMessali L, et al. Chronomodulated (Chrono) irinotecan (CPT) versus standard (STD) infusion in patients (pts) with metastatic colorectal cancer (MCC), a randomized multicenter trial. Eur J Cancer. 2001.

124. Garufi C, Bria E, Vanni B, Zappalà AMR, Sperduti I, Terzoli E. A phase II study of irinotecan plus chronomodulated oxaliplatin, 5-fluorouracil and folinic acid in advanced colorectal cancer patients. Br J Cancer. 2003

125. D. C, J. C, K. Y, Y. M, F. Y. Retrospective analysis of chronomodulated chemotherapy versus conventional chemotherapy with paclitaxel, carboplatin, and 5-fluorouracil in patients with recurrent and/or metastatic head and neck squamous cell carcinoma. Onco Targets Ther. 2013.

126. Bajetta E, Pietrantonio F, Buzzoni R, Ferrario E, Valvo F, Mariani L, et al. Chronomodulated capecitabine and adjuvant radiation in intermediaterisk to high-risk rectal cancer: A phase II study. Am J Clin Oncol Cancer Clin Trials. 2014.

127. Zhang PX, Jin F, Li ZL, Wu WL, Li YY, Long JH, et al. A randomized phase II trial of induction chemotherapy followed by cisplatin chronotherapy versus constant rate delivery combined with radiotherapy. Chronobiol Int. 2018,

128. Garufi C, Torsello A, Tumolo S, Ettorre GM, Zeuli M, Campanella C, et al. Cetuximab plus chronomodulated irinotecan, 5-fluorouracil, leucovorin and oxaliplatin as neoadjuvant chemotherapy in colorectal liver metastases: POCHER trial. Br J Cancer. 2010.

129. Squire T, Buchanan G, Rangiah D, Davis I, Yip D, Chua YJ, et al. Does chronomodulated radiotherapy improve pathological response in locally advanced rectal cancer? Chronobiol Int. 2017.

130. Goyal M, Shukla P, Gupta D, Bisht SS, Dhawan A, Gupta S, et al. Oral mucositis in morning vs. evening irradiated patients: A randomised prospective study. Int J Radiat Biol. 2009.

131. Chan S, Rowbottom L, McDonald R, Zhang L, Bjarnason GA, Tsao $\mathrm{M}$, et al. Could time of whole brain radiotherapy delivery impact overal survival in patients with multiple brain metastases? Ann Palliat Med. 2016.

132. Noh JM, Choi DH, Park H, Huh SJ, Park W, Seol SW, et al. Comparison of acute skin reaction following morning versus late afternoon radiotherapy in patients with breast cancer who have undergone curative surgical resection. J Radiat Res. 2014.

133. Chang L, Li L, Li W, Jiang M, Jv Y, Wang L, et al. Research on radiotherapy at different times of the day for inoperable cervical cancer. Int J Clin Pharmacol Ther. 2016.

134. Zhu L, Wang Q, Hu Y, Wang F. The circadian gene PER1 plays an important role in radiation-induced apoptosis and DNA damage in glioma. Asian Pacific J Cancer Prev. 2019.

135. Plikus M V., Vollmers C, De La Cruz D, Chaix A, Ramos R, Panda $\mathrm{S}$, et al. Local circadian clock gates cell cycle progression of transient amplifying cells during regenerative hair cycling. Proc Natl Acad Sci U S A. 2013.

136. Jiang W, Zhao S, Shen J, Guo L, Sun Y, Zhu Y, et al. The MIR135b-BMAL1-YY1 loop disturbs pancreatic clockwork to promote tumourigenesis and chemoresistance article. Cell Death Dis. 2018.

137. Solt LA, Wang Y, Banerjee S, Hughes T, Kojetin DJ, Lundasen T, et al. Regulation of circadian behaviour and metabolism by synthetic REVERB agonists. Nature. 2012.

138. Wagner PM, Monjes NM GM. Chemotherapeutic Effect of SR9009, a REV-ERB Agonist, on the Human Glioblastoma T98G Cells. ASN Neuro. 2019;11(1759091419892713).

139. Lin H-H, Robertson KL, Bisbee HA, Farkas ME. Oncogenic and Circadian Effects of Small Molecules Directly and Indirectly Targeting the Core Circadian Clock. bioRxiv. 2019. 